\title{
Comparative studies on regulation of SNARE complex formation by the SM protein Sly1p
}

\author{
PhD Thesis
}

\author{
in partial fulfillment of the requirements \\ for the degree "Doctor of Philosophy (PhD)" \\ in the Molecular Biology program \\ at the Georg August University Göttingen, \\ Faculty of Biology
}

submitted by

Fatma Esra Demircioglu

born in

Bursa, Turkey 
Thesis Committee Members:

Prof. Dr. Dirk Fasshauer

Dr. Stefan Eimer
Department of Cell Biology and Morphology

University of Lausanne

Switzerland

Institute of Chemistry and Biochemistry Freie University Berlin

Germany

European Neuroscience Institute (ENI)

Georg-August University Göttingen

Germany 
Herewith I declare, that I prepared the PhD Thesis "Comparative studies on regulation of SNARE complex formation by the SM protein Sly1p" on my own and with no other sources and aids than quoted.

Göttingen, 30.09.11

Fatma Esra Demircioglu 
"I do not know what I may appear to the world; but to myself I seem to have been only like a boy playing on the seashore, and diverting myself in now and then finding of a smoother pebble or a prettier shell than ordinary, whilst the great ocean of truth lay all undiscovered before me."

Sir Isaac Newton (1642-1727) English physicist, mathematician. 


\section{Table of contents}

TABLE OF CONTENTS i

LIST OF FIGURES iii

LIST OF TABLES $\quad$ v

ABBREVIATIONS vi vi vition

ACKNOWLEDGEMENTS vii

ABSTRACT $\quad$ ix

CHAPTER 1: INTRODUCTION

1.1 Vesicular transport hypothesis $\quad 1$

1.2 SNARE protein family 3

1.2.1 Essential role of SNAREs in vesicle fusion 3

$\begin{array}{ll}\text { 1.2.2 SNARE structure } & 5\end{array}$

1.2.3 Assembly of SNAREs $\quad 8$

$\begin{array}{ll}\text { 1.2.4 Fusion } & 12\end{array}$

1.2.5 Disassembly $\quad 14$

$\begin{array}{ll}\text { 1.2.6 SNARE specificity } & 15\end{array}$

1.3 The SM protein family $\quad 16$

$\begin{array}{ll}\text { 1.3.1 Essential role of SM proteins in vesicle fusion } & \mathbf{1 6}\end{array}$

$\begin{array}{ll}\text { 1.3.2 Functional studies on SM proteins } & 23\end{array}$

1.4 ER-to-Golgi transport in yeast and its regulation by Sly1p 29

1.4.1 Anterograde transport between the ER and Golgi in yeast $\quad 29$

1.4.2 Role of Sly1p in the ER-to-Golgi transport step 32

1.5 Aim of the work 36

CHAPTER 2: MATERIALS \& METHODS 37

2.1 Materials $\quad 37$

2.1.1 Chemicals 37

2.1.2 Enzymes, kits, bacterial strains and vectors 37

$\begin{array}{ll}\text { 2.1.3 DNA constructs } & 37\end{array}$

2.1.4 Peptides 38

2.1.5 Gene synthesis $\quad 39$

2.2 Methods 39

2.2.1 Protein expression and purification 39

2.2.2 Isothermal titration calorimetry (ITC) $\quad 42$

2.2.3 CD spectroscopy $\quad 42$

2.2.4 EPR spectroscopy $\quad 44$

2.2.5 Fluorescence spectroscopy $\quad 45$

2.2.6 Other methods $\quad 47$

CHAPTER 3: RESULTS 49

3.1 Biochemical characterization of Sly1p interaction with the ER-Golgi SNAREs 49

3.1.1 Elucidating the conformation of individual Sed5 $p \quad 49$ 
3.1.2 Analysis of the interaction between Sly1p and Sed5p

3.1.3 Does Sly1p bind to the ER-Golgi SNARE complex? $\quad 61$

3.1.4 Sly1p interacts with another syntaxin on the retrograde pathway of ER-Golgi transport

3.2 Monitoring assembly of the ER-Golgi SNAREs

3.2.1 Investigating interactions between the ER-Golgi SNAREs by CD spectroscopy

3.2.2 Monitoring assembly kinetics of the ER-Golgi SNAREs by fluorescence anisotropy

3.2.3 Qabc subcomplex of ER-Golgi SNAREs can be stabilized to serve as an assembly intermediate for SNARE complex formation

3.2.4 A transient QabR subcomplex allows rapid assembly of the ER-Golgi SNAREs

3.3 Monitoring the effect of Sly1p on assembly of the ER-Golgi SNAREs

3.3.1 Sly1p promotes SNARE assembly through the transient QabR subcomplex

3.3.2 Assembly and regulation of ER-Golgi SNAREs, followed by FRET experiments

3.3.3 Regulation of SNARE assembly by the mutants of Sed5p and Sly1p

4.1 Biochemical characterization of individual Sed5p

and the Sly1p/Sed5p complex

4.2 Assembly of the ER-Golgi SNAREs

4.3 Regulation of the assembly of ER-Golgi SNAREs by Sly1p 103

4.4 Do the two binding sites on Sly1p work

together for regulating SNARE assembly?

CHAPTER 5: SUMMARY \& CONCLUSIONS

A.1 Sequence alignment of Sed5p from fungi

A.3 Sly1p does not enhance the assembly kinetics

of the Qabc and the QacR complexes 


\section{List of figures}

FIGURE 1.1 A short glimpse at vesicle budding and fusion 2

FIGURE 1.2 Structure of SNAREs and the SNARE core complex $\quad 7$

FIGURE 1.3 Conformational cycle of SNAREs during a vesicle fusion event 13

FIGURE 1.4 Crystal structures of Munc18a (A) and of Sly1p (B)

in complex with their cognate syntaxins

FIGURE 1.5 Tentative assignment of the involved sets of SNAREs in S. cerevisiae $\quad 30$

FIGURE 1.6 Comparison of Sly1p with Munc18a and the Sly1p homologues 34

FIGURE 2.1 Operation of a CD spectrometer 43

FIGURE 2.2 The spin-labeling reaction of MTSL 45

FIGURE 3.1 Schematic diagram of the domain structure of Sed5p $\quad 50$

FIGURE 3.2 Secondary structure characterization of Sed5p fragments using CD spectroscopy

FIGURE 3.3 Biochemical analysis of the interaction between the Sed5p

SNARE motif and the Sed5p N-terminal domain

FIGURE 3.4 Calorimetric titration of Sed5p SNARE motif with the Sed5p N-terminal domain

FIGURE 3.5 Biochemical analysis of Sly1p interaction with truncated Sed5p variants $\quad 55$

FIGURE 3.6 EPR spectra of individual Sed5p or in complex with Sly1p $\quad 58$

FIGURE 3.7 Calorimetric titration of Sly1p with the Sed5p (1-210)/ (211-320) complex

FIGURE 3.8 Calorimetric titration of Sly1p with Sed5p variants carrying the F10A mutation

FIGURE 3.9 Calorimetric titration of Sly1p with ER-Golgi SNARE complexes $\quad \mathbf{6 2}$

FIGURE 3.10 Calorimetric titration of Sly1p with Ufe1p N-peptide

FIGURE 3.11 Displacement titrations of Sed5p (1-320) and Sed5p (1-210) with Sly1p, using Ufe1p (1-21) as a competitive ligand

FIGURE 3.12 Interaction of ER-Golgi SNAREs observed by CD spectroscopy

FIGURE 3.13 Assembly kinetics of complexes formed between ER-Golgi SNAREs, followed by CD spectroscopy

FIGURE 3.14 Assembly kinetics of ER-Golgi SNAREs observed by fluorescence anisotropy

FIGURE 3.15 A stabilized Qabc-complex of ER-Golgi SNAREs can serve as acceptor for Sec $22 p$ 
FIGURE 3.16 Assembly kinetics of ER-Golgi SNAREs, observed after pre-incubation of unlabeled SNAREs

FIGURE 3.17 The transient QabR subcomplex exists in a dynamic equilibrium with its components

FIGURE 3.18 Assembly kinetics of ER-Golgi SNAREs in the presence of Sly1p

FIGURE 3.19 Assembly kinetics of ER-Golgi SNAREs, observed after pre-incubation of unlabeled SNAREs in the presence of Sly1p

FIGURE 3.20 Equilibrium concentration of the QabR intermediate can be shifted in the presence of Sly1p

FIGURE 3.21 Sly1p does not excessively enhance the assembly kinetics of the QabR-intermediate

FIGURE 3.22 Assembly and regulation of ER-Golgi SNAREs, followed by FRET assays

FIGURE 3.23 SNARE assembly kinetics followed by fluorescence anisotropy and FRET, in the presence of the F10A mutant of Sed5p

FIGURE 3.24 SNARE assembly kinetics followed by fluorescence anisotropy and FRET, in the presence of the $\Delta \mathrm{N}$-mutant of Sed5p

FIGURE 3.25 SNARE assembly kinetics in the presence of a Sed5p variant containing a modified linker

FIGURE A.1 Sequence alignment of Sed5p from fungi

FIGURE A.2 Purification of stable complexes formed between the ER-Golgi SNAREs

FIGURE A.3 Sly1p does not enhance the assembly kinetics of the Qabc and the QacR complexes 


\section{List of tables}

TABLE 1.1 The SM proteins that are discussed in this study 17

TABLE 2.1 DNA constructs used in the study 38

TABLE 2.2 Composition of SDS-PAGE and native gels 48

TABLE 3.1 Thermodynamic parameters obtained by all ITC experiments in this study 56 


\section{Abbreviations}

\begin{tabular}{|c|c|}
\hline AAA + & ATPases associated with various cellular activities \\
\hline ATP & Adenosine triphosphate \\
\hline cal & calorie \\
\hline $\mathrm{CD}$ & circular dichroism \\
\hline cDNA & complementary DNA \\
\hline DMF & N, N Dimethylformamide \\
\hline DNA & Deoxyribonucleic acid \\
\hline Dnase & Deoxyribonuclease \\
\hline DTT & Dithiothreitol \\
\hline EDTA & Ethylene diamine tetraacetic acid \\
\hline EPR & electron paramagnetic resonance \\
\hline Fig. & Figure \\
\hline FRET & Förster resonance energy transfer \\
\hline GST & Glutathion S-Transferase \\
\hline IPTG & Isopropyl- $\beta$-D-thiogalactoside \\
\hline $\mathrm{h}(\mathrm{s})$ & Hour(s) \\
\hline ITC & Isothermal titration calorimetry \\
\hline $\mathrm{K}_{\mathrm{d}}$ & Dissociation constant \\
\hline $\mathrm{kDa}$ & kilo Dalton \\
\hline $\mathrm{kV}$ & kilovolt \\
\hline LB & Luria Bertani \\
\hline $\mathrm{mA}$ & milliampere \\
\hline $\mathrm{mF}$ & millifarad \\
\hline $\min (\mathrm{s})$ & minute(s) \\
\hline $\mathrm{ml}$ & milliliter \\
\hline $\mathrm{mM}$ & millimolar \\
\hline $\mathrm{nm}$ & nanometer \\
\hline MTSL & methanethiosulfonate \\
\hline MWCO & molecular weight cut off \\
\hline NMR & nuclear magnetic resonance \\
\hline $\mathrm{Ni}^{2+}$-agarose & Nickel-agarose \\
\hline NSF & N-ethylmaleimide-sensitive factor \\
\hline OD & optical density \\
\hline OG & Oregon green 488 iodoacetamide \\
\hline PAGE & Polyacrylamide gel electrophoresis \\
\hline PBS & Phosphate buffered saline \\
\hline PCR & Polymerase chain reaction \\
\hline PMSF & Phenylmethylsulphonylfluoride \\
\hline $\mathrm{pH}$ & negative logarithm of $\mathrm{H}^{+}$concentration \\
\hline rpm & revolutions per minute \\
\hline RT & room temperature \\
\hline SDS & Sodium dodecyle sulfate \\
\hline SNAP & Soluble- NSF-attachment protein \\
\hline SNAP-25 & Synaptosome associated protein of $25 \mathrm{kDa}$ \\
\hline SNARE & SNAP-receptor \\
\hline Syb & Synaptobrevin \\
\hline Syx & Syntaxin \\
\hline TB & terrific broth \\
\hline TCEP & Tris(2-carboxyethyl)phosphine \\
\hline TEMED & $\mathrm{N}, \mathrm{N}, \mathrm{N}^{\prime}, \mathrm{N}^{\prime}$-Tetramethylethylene diamine \\
\hline TR & Texas Red 520 maleimide \\
\hline Tris & Tris(hydroxymethyl)-aminomethane \\
\hline UV & Ultraviolet \\
\hline $\mathrm{V}$ & Volt \\
\hline VAMP & vesicle associated membrane protein \\
\hline $\mathrm{v} / \mathrm{v}$ & volume/ volume \\
\hline $\mathrm{w} / \mathrm{v}$ & weight/ volume \\
\hline
\end{tabular}




\section{Acknowledgements}

I am indebted to Prof. Dr. Dirk Fasshauer for his expert guidance in this project. I am deeply thankful to him for useful discussions, tips and also for providing a warm working atmosphere in our small group.

I am grateful to Prof. Dr. Reinhard Jahn for his generous support. Working in the department of neurobiology and being nearby almost all equipment that I need, was a privilege.

I extend my gratitude to Prof. Dr. Markus Wahl and Dr. Stefan Eimer for being in my thesis committee and for their interest, advice and support during the course of the project.

I am indebted to Göttingen Graduate School for Neurosciences and Molecular Biosciences (GGNB) and Molecular Biology coordination offices, particularly to Dr. Steffen Burkhardt, Kerstin Grueniger and Kirsten Poehlker, for their endless assistance through all administrative work that I went through. I further acknowledge the GGNB graduate school as being a recipient of the GGNB excellence fellowship.

I am thankful to Prof. Dr. Marina Bennati and Dr. Giusseppe Sicoli for their help and advice in EPR experiments.

I am deeply thankful to Dr. Pawel Burkhardt for his expert advice and encouraging discussions. The first two years would not have been that joyful without him.

I must acknowledge Dr. Gottfried Mieskes for his endless patience and generous help for all members of the department of neurobiology.

Thanks to Dr. Nickias Kienle, Emilio Iraheta, Dr. Katrin Wiederhold, Dr. Tobias Klöpper, Dr. Ulrike Winter, Dr. Anand Radhakrishnan and Dr. Alexander Stein for their help, for useful suggestions, for being wonderful colleagues and for all amusement that we had.

I am thankful to Dr. Geert van den Bogaart for tips and suggestions. He kept up my inspiration on the project by helpful discussions. 
Thanks to Wolfgang Berning-Koch for his technical assistance and for making me always smile.

Thanks to my new labmates Dr. Matias Hernandez, Halenur Yavuz, Julia Preobraschenski and Dr. Dominika Czernik for their help and friendship -in particular at hard times.

Thanks to all members of the Department of Neurobiology for their friendship and for the wonderful working atmosphere.

Thanks to Dr. Chao-Hua Huang for our lunch discussions on every friday.

Thanks to Dr. Pınar Öz and Buket Özkaya for being my special friends in Göttingen.

Thanks to Dr. Ramya Nair, Dario Arcos-Diaz and Dr. Peer Hoopmann for their friendship, for all the joy.

Thanks to my family and to everybody that I accept as my family. They know who they are...Particularly to my mother, since without her support on the other end of the telephone line, I would perhaps not make it this far.

And thanks to Nikhil for his love. 


\section{Abstract}

Sec1/ Munc18 (SM) proteins are indispensible regulators of intracellular membrane fusion. In general, the high-affinity binding partners of SM proteins are Qa-SNAREs (syntaxins). Despite the high structural homology among SM proteins, different modes are proposed for their association with syntaxins. Neuronal Munc18a binds to a "closed conformation" formed by Syntaxin 1a, whereas several SM proteins interact only with the $\mathrm{N}$-peptide motifs of their cognate syntaxins. On the other hand, recent findings suggest that SM proteins might exert regulatory role, acting on both the N-peptide motif and the closed conformation. So far, yeast Sly1p, the SM protein of ER-Golgi trafficking, is considered to interact solely with the N-peptide portion of its cognate syntaxin Sed5p. In light of the proposed common binding model, I investigated the Sly1p/ Sed5p interaction using biochemical and biophysical tools. My findings demonstrated that N-peptide binding is the major contributor to the high affinity, yet the remainder of Sed5p contributes as well. I showed that individual Sed5p can adopt a closed conformation, which raised the question whether Sly1p can assist Sed5p to switch to a SNARE-accessible configuration. To address this question, I developed kinetic assays that monitor the assembly of ER-Golgi SNAREs. I observed that Sly1p accelerates the speed of SNARE complex formation, suggesting a positive regulatory role for the SM protein. Further analysis revealed that Sly1p promotes SNARE assembly by supporting and/or stabilizing a folding intermediate required for SNARE complex formation. Next, I evaluated whether Sly1p makes use of the the two binding sites of Sed5p to be able to orchestrate the SNARE machinery. The current analysis suggests that the N-peptide binding plays a crucial role for the regulatory function of Sly1p. My findings suggest that Sly1p and possibly other SM proteins might regulate the SNARE assembly by acting on the two binding sites of their syntaxins, in a yet undefined manner. 


\section{Chapter 1}

\section{Introduction}

\subsection{Vesicular transport hypothesis}

Over 30 years ago, it was the work by Palade and co-workers which revealed that the intracellular secretory machinery consists of a series of transport steps between membrane-enclosed compartments (Palade, 1975). The compartment where membranebound and soluble proteins and lipids are made is the endoplasmic reticulum (ER). Newly synthesized cargo is then delivered to from ER to the Golgi complex for maturation and processing. From the Golgi complex, the cargo molecules are segregated into distinct organelles. Some molecules that are destined to be degraded move from the Golgi to lysosomes through intermediate compartments called late endosomes. Molecules that are secreted from the cell do so by a process called exocytosis. For each of the transport steps between different compartments, the cell utilizes small, membraneenclosed vesicles which act as shuttles. These vesicles "bud" from a "donor" compartment after selective incorporation of cargo, and the vesicle is then targeted to an "acceptor" compartment where it "fuses" to unload its cargo. The directionality of vesicular transport is reversed as well to retrieve the transport machinery components or missorted cargo from the acceptor compartment to donor. Transport in the reverse direction also includes cargo uptake from the extracellular milieu, during which cargo is taken up by the plasma membrane by endocytosis and then transported to early endosomes and lysosomes (Bonifacino and Glick, 2004, Lodish, 2004).

Beginning in the late 1970's, the molecular mechanisms that underlie vesicular transport have slowly become elucidated. In early studies, various components involved in vesicle 


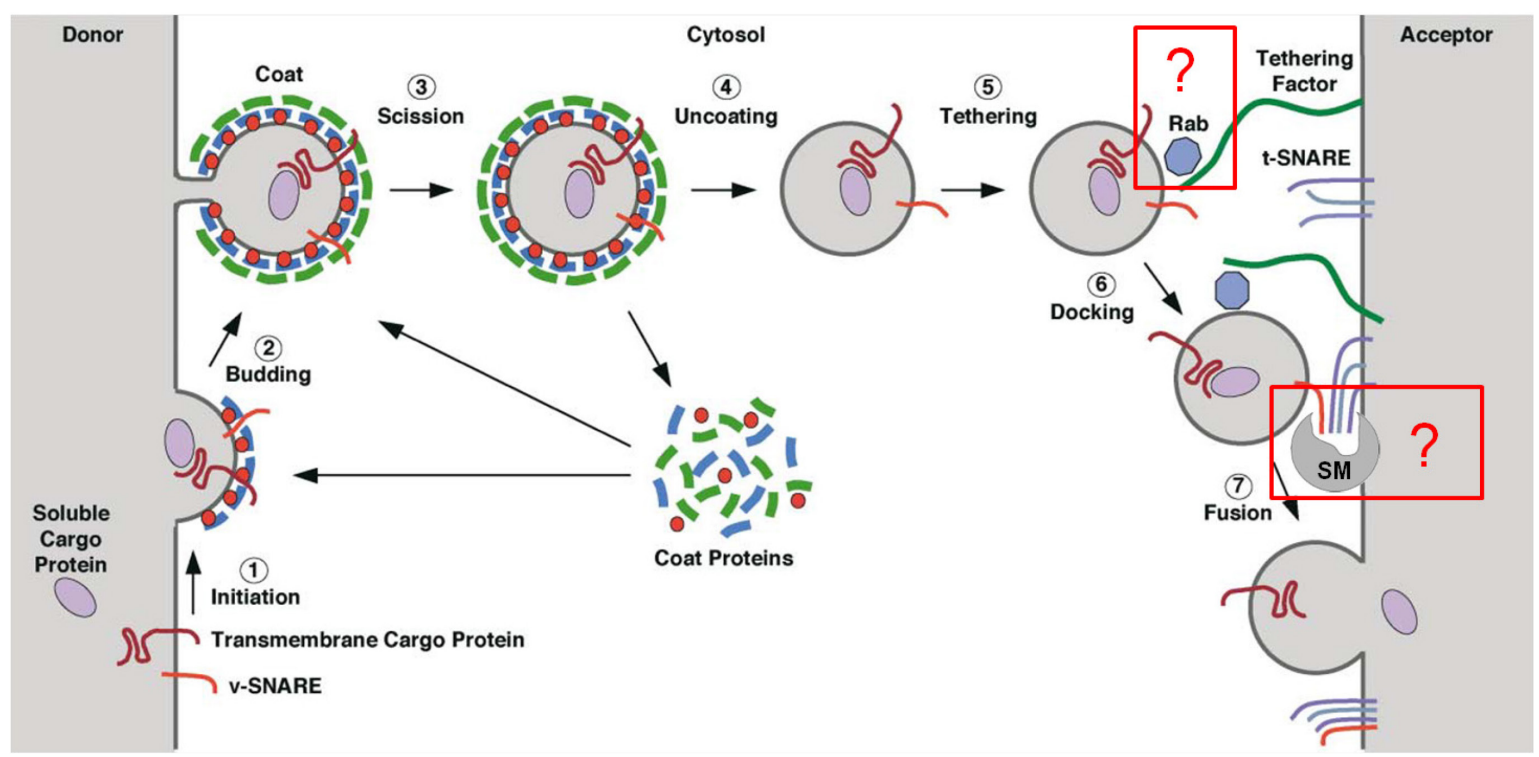

Figure 1.1: A short glimpse at vesicle budding and fusion

Vesicle budding is initiated with recruitment of coat proteins from the cytosol. The coat proteins start deforming membrane patches into buds while recognizing and recruiting the transmembrane cargo proteins as well as fusion proteins called SNAREs. Meanwhile, soluble protein cargo is also recognized and sorted to vesicle buds by transmembrane cargo receptors (step 1). After the complete assembly of the coat components, the membrane curvature increases (step 2) and scission occurs by either coat proteins or accessory proteins (step 3). Following its detachment from the donor membrane, the vesicle sheds its coat (step 4). Then, the "naked" vesicle moves until reaching its target possibly guided by cytoskeleton. The tethering of the vesicle to the acceptor membrane is thought to occur with the aid of Rab family GTPases and tethering factors (step 5). Next, the vesicle docks at a specific position on the membrane with the help of SNAREs and accessory proteins (step 6). Finally, the vesicle fusion is thought to occur by formation of a four-helical bundle between the SNARE proteins residing on opposing membranes (step 7). The terms v-SNARE (vesicle-membrane SNARE) and t-SNARE (target-membrane SNARE) arise from the functional classification of SNAREs. Due to their unknown precise roles, Rab and the SM (Sec1/ Munc18-like) proteins are highlighted with question marks. (Picture is modified from Bonifacino and Glick, 2004).

budding and fusion were identified using yeast genetics or by in vitro screening. By genetically manipulating yeast, Schekman and colleagues have isolated temperaturesensitive "sec" mutants, that are defective in protein secretion. This allowed identification of 23 SEC genes, the protein products of which play major roles in multiple stages of the secretory pathway (Novick et al., 1980). During approximately the same time, the first in vitro studies were initiated by Rothman and colleagues, who devised a cell-free assay to measure the transport within the mammalian Golgi complex (Balch et al., 1984). It later became apparent that the molecular machinery responsible for vesicular trafficking, including budding and fusion, were in fact the same in both these systems and thus it must have been broadly conserved throughout evolution. A current and widely accepted model for vesicular transport is summarized in figure 1.1. 


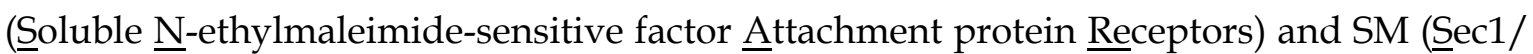
Munc18-like) proteins. In the next sections, the role and function of both SNAREs and SM proteins will be briefly reviewed and dicussed.

\subsection{SNARE protein family}

\subsubsection{Essential role of SNAREs in vesicle fusion}

At present, SNARE proteins are thought to be the key fusion machinery of vesicular transport. SNAREs seem to mediate vesicle fusion in almost all trafficking steps of the secretory pathway although there are some notable exceptions including the homotypic fusion of mitochondria and peroxisomes (Hales and Fuller, 1997, Malka et al., 2005, Meeusen et al., 2004, Titorenko et al., 2000, Titorenko and Rachubinski, 2000).

The role of SNAREs in fusion has been realized much later then their initial discovery. The first candidate protein involved in fusion was called NSF (N-ethylmaleimide Sensitive Factor), which was identified using an innovative cell-free assay developed by Rothman and colleagues. NSF was initially thought to be mainly responsible for mediating fusion within Golgi cisternea (Glick and Rothman, 1987), and was later found to be implicated in other vesicle fusion steps in the secretory pathway (Beckers et al., 1989,

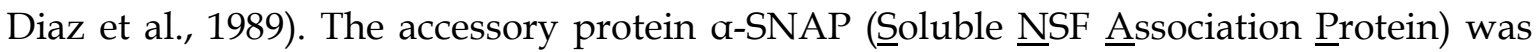
also identified as a co-factor which binds NSF to membranes, however, it was not clear at the time how a-SNAP was specifically recruited to membranes. When Söllner and colleagues fractionated a brain lysate using NSF/a-SNAP as an affinity reagent, three membrane-associated proteins were isolated, which were found to be responsible for membrane recruitment (Sollner et al., 1993b). These proteins were then termed "SNAPreceptors" or SNAREs. Further analysis of the SNAREs revealed that they corresponded to be the synaptic proteins Synaptobrevin (VAMP-2), SNAP-25 (25-kDa Synaptosome Associated Protein) and Syntaxin 1a, which were already suspected of being involved in the vesicular release of neurotransmitters (Walch-Solimena et al., 1993). For instance, they were already known to be targets of Clostridial neurotoxin (CNT) light-chain proteases (Blasi et al., 1993, Schiavo et al., 1992, Schiavo et al., 1993), which cause disruption of synaptic vesicle docking and fusion and are responsible for muscle paralysis in botulism 
and tetanus diseases.

Despite having identified the main components of the fusion machinery, questions remained regarding the exact role of each. A prevalent view was that SNAREs were recruitment or docking factors which were responsible for conveying specificity to the fusion process, whereas NSF was postulated to use energy from ATP and to actually catalyze the fusion reaction (Sollner et al., 1993a). However, an alternative hypothesis was proposed based on the observation that Syntaxin 1a, SNAP-25 and Synaptobrevin form a coiled-coil which align in parallel with their transmembrane domains next to each other (Hanson et al., 1997, Lin and Scheller, 1997). This led to a more intuitive concept that SNAREs located on opposing membranes could associate at one end and "zipper" up towards the membranes, pulling them closer together and thus initiating fusion. Supporting this hypothesis, Rothman and colleagues demonstrated that SNARE proteins reconstituted on proteoliposomes are capable of fusing membranes at physiological temperature without the need for any additional factors (Weber et al., 1998). This experiment established SNAREs as the minimal fusion machinery.

It is now known that SNAREs residing on opposing membranes associate with other SNAREs by forming a four-helical bundle called the "SNARE complex". According to current thinking, the free energy released upon formation of the complex is used to overcome the energy barrier required for fusion (Hanson et al., 1997, Lin and Scheller, 1997). Contrary to their original attributed role, NSF and its accessory protein a-SNAP disassemble the highly stable four-helical SNARE complex using energy from ATP in order to recycle SNAREs after fusion has been completed (Sollner et al., 1993a).

At present, several physiological studies have confirmed the crucial roles of neuronal SNAREs during synaptic vesicle fusion. For instance, in Synaptobrevin knockout mice, the rate of both spontaneous and action potential-triggered synaptic vesicle exocytosis is partially decreased (Schoch et al., 2001). However, the loss of Synaptobrevin in mice is most likely compensated by a Synaptobrevin homologue, Cellubrevin (McMahon et al., 1993). In double-knockout mice lacking both Synaptobrevin and Cellubrevin neurosecretion is completely blocked, while vesicular biogenesis and docking remain unaffected (Borisovska et al., 2005). Studies in SNAP-25 knockout mouse show that action potential-triggered vesicle exocytosis is completely abolished in these organisms (Washbourne et al., 2002). 
Further support for an essential role of SNAREs in vesicle fusion has come from genetic studies in yeast. A wealth of evidence shows that the neuronal SNAREs Synaptobrevin, Syntaxin 1 and SNAP-25 all have analogues in yeast and that they are responsible for the various intracellular transport pathways (Gerst et al., 1992, Aalto et al., 1993, Becherer and Jones, 1992, Ferro-Novick and Jahn, 1994). A more detailed account of these findings is beyond the scope of this introduction and several excellent reviews are available (FerroNovick and Jahn, 1994, Pfeffer, 1996, Jahn and Scheller, 2006).

\subsubsection{SNARE structure}

The characteristic feature of all SNARE proteins is the SNARE (H3) motif, a conserved stretch of 60-70 amino acids which are arranged in heptad repeats. SNAREs are membrane-attached, in most cases, via a single transmembrane domain at their Cterminal end. The transmembrane domain is connected to the H3 motif with a short linker. A small number of SNAREs, on the other hand, are anchored to the membrane via hydrophobic post-translational modifications. For instance, neuronal SNAP-25 has two SNARE motifs that are connected by a flexible linker which is palmitoylated (Jahn and Scheller, 2006). Another example is the yeast Ykt6p, in which a CAAX box on its Cterminus is farnesylated (McNew et al., 1997).

According to NMR (Nuclear $\underline{\text { Magnetic }}$ Resonance) and CD (Circular Dichroism) experiments, the individual SNARE motifs are unstructured in solution (Fasshauer et al., 1997a, Fasshauer et al., 1997b, Fiebig et al., 1999, Hazzard et al., 1999). It needs to be mentioned, however, that, the structural configuration of the SNARE motifs in vivo is being debated due to the influence of the membrane environment and the accessory proteins (Fasshauer, 2003). In solution, major structural rearrangements occur during SNARE assembly, as individual SNAREs form a highly stable, hetero-oligomeric fourhelical bundle. The protease-resistant minimal portion of this complex is termed as "SNARE core complex" (Fasshauer et al., 1998a). X-ray crystal structures of distantly related SNARE core complexes show remarkable resemblance, suggesting that the core complex is an evolutionary hallmark of the fusion machinery (Sutton et al., 1998, Antonin et al., 2002, Zwilling et al., 2007, Strop et al., 2008).

Initially, SNAREs were functionally classified as v-SNAREs (vesicle-membrane SNAREs) and t-SNAREs (target-membrane SNAREs) according to their preferential localization on 
a donor or an acceptor compartment (Sollner et al., 1993a). However, this terminology is sometimes ambiguous. For instance, yeast Sec22p which functions during both anterograde and retrograde transport between ER and Golgi, is classified as a t-SNARE in the anterograde pathway, whereas as a v-SNARE in the retrograde pathway (Dilcher et al., 2003, McNew et al., 2000, Lewis et al., 1997, Burri et al., 2003). Structural and amino acid sequence analysis of SNAREs has been used for an alternative classification after the crystal structure determination of the synaptic SNARE core complex (Figure 1.2 A). The synaptic core complex consists of parallel a-helices arranged in 16 stacked layers of interacting side-chains that are mostly hydrophobic. An exception is the central, so-called " 0 " layer, that contains three glutamine $(Q)$ residues, one contributed by Syntaxin 1a and two by SNAP-25 and one arginine (R) residue contributed by Synaptobrevin. Regarding the side chains that they provide in several other layers, all four SNAREs are distinct, each representing one major SNARE class. Since the sequence conservation is exceptionally high across SNAREs of different pathways and species, the amino acid composition of the central layer is the basis of the biochemical classification of SNAREs into Q- and RSNAREs, while Q-SNAREs are further divided into Qa, Qb and Qc (Fasshauer et al., 1998b). A more elaborate classification scheme has been recently built from those 4 major classes with 20 new subclasses. The new subclasses reflect the participation of SNAREs in different trafficking steps and all eukaryotic SNAREs are thought to originate from this repertoire (Kloepper et al., 2007).

\section{$N$-terminal domains}

SNAREs have different types of N-terminal domains, which are connected to the H3 motif via linker segments. Mostly Qa-SNAREs (also termed syntaxins) and also some of the Qband Qc-SNAREs contain N-terminal antiparallel three-helix bundles, termed "Habc" domains (Jahn and Scheller, 2006). An interesting exception is the yeast Qc-SNARE Vam7, which uniquely has a "Phox- homology (PX) domain", that is responsible for membranebinding (Figure 1.2 B) (Lu et al., 2002). Some R-SNAREs have profilin-like folds or longin domains on their N-terminal ends (Figure 1.2 B) (Misura et al., 2002, Dietrich et al., 2003, Gonzalez et al., 2001, Tochio et al., 2001). On the other hand, the evolutionary newer brevin subfamily of R-SNAREs, e.g., Synaptobrevin, lacks N-terminal domains (Rossi et al., 2004). 

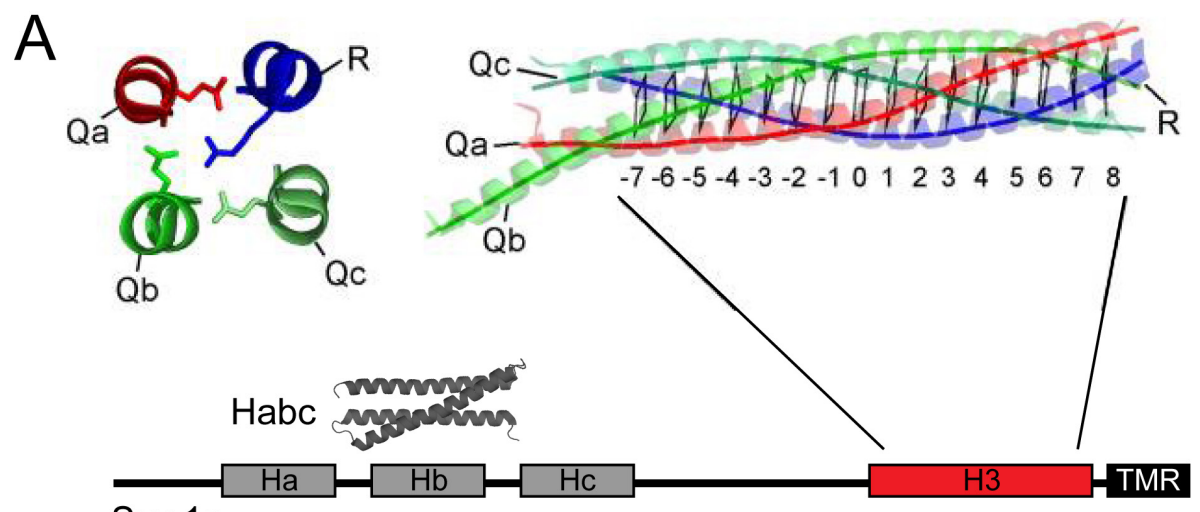

Syx $1 \mathrm{a}$

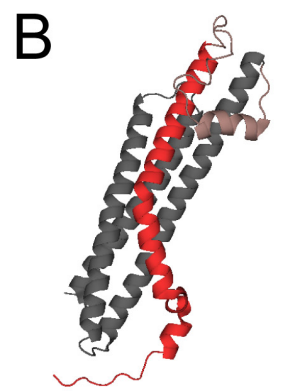

closed

Syntaxin $1 a$

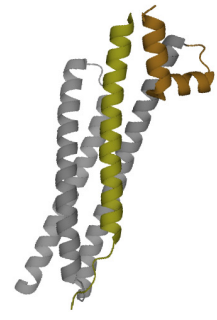

closed

Sso1p

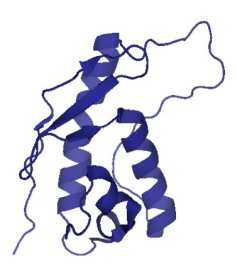

PX

domain

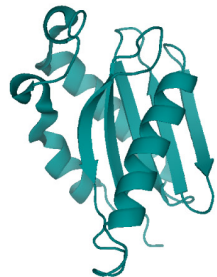

longin

domain

\section{Figure 1.2: Structure of SNAREs and the SNARE core complex}

A. On the top right, a ribbon diagram of the neuronal SNARE core complex is shown. The SNARE (H3) motifs of Syntaxin 1a (Qa: red), SNAP-25 (Qbc: green) and Synaptobrevin (R: blue) are in different colours and the helical axes are highlighted by lines. The layers (-7 to 8$)$ of the core bundle are colored in black. On the top left, the structure of the central " 0 " layer is shown. Below, the domain architecture of Syntaxin 1a (Syx 1a) is shown. The structure of the N-terminal Habc domain of Syx1a is also displayed (Lerman et al., 2000). Picture is adapted from Kloepper et al., 2007. B. Closed conformation adopted by syntaxins is shown in two examples: Syntaxin 1a (Misura et al., 2000) and Sso1p (Munson et al., 2000). Closed conformation of Syntaxin 1a was solved as part of the Munc18a/ Syx 1a complex. Habc helices are shown in grey, the SNARE motif of Syx1a is in red and that of Sso1p is in yellow. The linker helices between the Habc and the H3 motifs are displayed in pink and brown for Syx1a and Sso1p, respectively. On the right, N-terminal domains of nonsyntaxin-SNAREs are shown. PX-domain of the Qc-SNARE Vam7 (Lu et al., 2002) is in blue and the profilin or longin domain of the R-SNARE Ykt6 (Tochio et al., 2001) is in cyan.

Some SNAREs can adopt a so-called "closed conformation" such that the Habc domain folds back onto the N-terminal half of the SNARE motif. Syntaxin 1a and the yeast exocytic syntaxin, Sso1p are such examples (Figure 1.2 B). The closed conformation is thought to render the H3 motif inaccessible for partner SNAREs, thus, prior to SNARE complex assembly syntaxins are believed to be in open conformation (Pevsner et al., 1994, Misura et al., 2000, Nicholson et al., 1998). In closed form of Sso1p, the linker domain lying between the Habc and the SNARE motifs, seems to form the major block for binding of partner SNAREs (Nicholson et al., 1998). Conformation of the single Syntaxin 1a is controversial. According to a nuclear magnetic resonance (NMR) study, the isolated 
Syntaxin 1a adopts a weak closed conformation, whereas a single molecule fluorescence resonance energy transfer (FRET) study suggests that about $70 \%$ of Syntaxin 1a molecules are in open conformation in solution (Chen et al., 2008, Margittai et al., 2003b). However, Syntaxin 1a has been shown to adopt a tight closed conformation upon binding to the neuronal SM protein Munc18a (Misura et al., 2000). It should be noted that, some other Qa-SNAREs are suggested to exist only in open conformations (Dulubova et al., 2001, Yamaguchi et al., 2002, Dulubova et al., 2002). Thus, the question of what the functional significance of the closed conformation is, still being debated. Nevertheless, the Nterminal domain of yeast Sso1p seems to be essential for cell viability (Munson et al., 2000). In addition, a knockin/knockout mice expressing only an open-variant of Syntaxin 1 have abnormally enhanced synaptic vesicle fusion, which leads to generalized seizures and mortality at 2 to 3 months of age (Gerber et al., 2008). These reports suggest that the interaction between the N-terminal Habc domain and the SNARE motif might be critical for proper SNARE function and fusion.

Another yet to be addressed question is whether the Habc domain interacts with the core complex after SNAREs have assembled into a four-helix bundle. Thermal denaturation of synaptic and yeast exocytic SNARE complexes has revealed that the Habc domain is loosely connected with the SNARE core bundle once the SNARE complex has formed (Fasshauer et al., 1997b, Rice et al., 1997, Nicholson et al., 1998). In addition, electron paramagnetic resonance (EPR) studies show that the linker region between the Habc domain and Syntaxin 1a is highly flexible when syntaxin is part of the SNARE complex (Margittai et al., 2003a). The possible significance of this state and its role with accessory proteins will be discussed in subsequent sections.

\subsubsection{Assembly of SNAREs}

Owing to topological reasons, SNARE assembly is thought to proceed from the N-termini of the proteins towards the membrane-proximal C-termini during fusion. Several lines of evidence are consistent with a directional "zippering" model for SNARE assembly (Hanson et al., 1997, Fasshauer et al., 1998b, Fiebig et al., 1999). For instance, a monoclonal antibody against the N-terminal portion of SNAP-25 inhibits SNARE complex formation in vitro and in vivo (Xu et al., 1999). In addition, N-terminal truncations of SNAP-25 and the SNARE motif of Syntaxin 1a significantly slow down SNARE assembly in vitro, 
whereas C-terminal truncations have almost no effect. Interestingly, neither N- nor Cterminally truncated Synaptobrevin alters assembly kinetics (Fasshauer and Margittai, 2004), suggesting N-terminal assembly of Q-SNAREs is a prerequisite for initiation of the assembly of the four-helix bundle.

In vitro studies on yeast and synaptic SNARE complexes suggest that SNARE assembly is an ordered, sequential reaction rather than a random collision of the $\mathrm{H} 3$ motifs. For instance, pre-assembly of the Q-SNAREs seems to facilitate exocytic yeast SNARE complex formation such that Sso1p is thought to interact with Sec9p (SNAP-25 homologue) before Snc2p (Synaptobrevin homologue) can bind (Nicholson et al., 1998). Strong evidence for a stepwise assembly of neuronal SNAREs comes from denaturation experiments, where it has been shown that the SNARE complex does not refold at conditions where unfolding occurs (Fasshauer et al., 2002). This suggests that the disassembled and assembled states of SNAREs are most likely separated by formation of a folding intermediate. A kinetic analysis of the refolding process implicates that the intermediate acceptor complex could be a Syntaxin/ SNAP-25 complex (Fasshauer et al., 2002). This is consistent with the requirement of an initial N-terminal assembly of the QSNAREs as discussed previously (Fasshauer and Margittai, 2004).

Whereas yeast Sso1p and Sec9p form a 1:1 complex in solution, assembly of Syntaxin 1a and SNAP-25 results in a complex in 2:1 stoichiometry (Fiebig et al., 1999, Fasshauer et al., 1997b, Nicholson et al., 1998). The structure of this so-called 2:1 binary complex appears to be virtually identical to that of the ternary SNARE complex, except that Synaptobrevin is substituted with a second copy of Syntaxin 1a (Margittai et al., 2001, Xiao et al., 2001, Zhang et al., 2002). Since the Synaptobrevin binding site is occluded, the 2:1 Syntaxin 1a/SNAP-25 complex intuitively seems to be an off-pathway for SNARE complex formation. Supporting the idea that a 1:1 Syntaxin 1a/ SNAP-25 complex is an onpathway intermediate, binding of Synaptobrevin has been found to be enhanced when the binary complex is pre-formed with an excess of SNAP-25 (Fasshauer and Margittai, 2004). The structural configuration of this putative 1:1 acceptor complex is difficult to investigate since it is transient and readily converts to the 2:1 form. However, when Syntaxin 1a and SNAP-25 are assembled in the presence of a C-terminal Synaptobrevin fragment, a stable 1:1 complex can be formed. The stability of this complex is conferred by the C-terminal Synaptobrevin fragment, which occupies the Synaptobrevin assembly site and prevents a second Syntaxin 1a from binding, but the N-terminal binding site of Synaptobrevin 
remains free. Additionally, it may assist in structuring the N-terminal region of the QSNAREs (Pobbati et al., 2006). This 1:1 " $\Delta \mathrm{N}$-complex" provides rapid ternary SNARE complex formation (within $1 \mathrm{~min}$ at $1 \mu \mathrm{M}$ ) in vitro, which dramatically contrasts to the hour-timescale assembly kinetics without stabilization. It is likely that this slow assembly kinetics is partly due to the slow, rate-limiting interaction of Syntaxin 1a and SNAP-25 $\left(\sim 6000 \mathrm{M}^{-1} \mathrm{~s}^{-1}\right)$, and partly also due to the short lifetime of the 1:1 acceptor complex (Fasshauer and Margittai, 2004).

The existence of a pre-assembled acceptor complex intermediate before the arrival of an action potential would considerably speeds up ternary SNARE complex assembly. However, how an intermediate acceptor complex is formed and stabilized for neurotransmitter release is still largely unknown. Recently, using single-molecule fluorescence, the dynamics of 1:1 Syntaxin 1a/ SNAP-25 complex has been followed and different states have been observed such that one of the SNAP-25 helices either dissociates from or stays bound to the complex (Weninger et al., 2008). Addition of several accessory proteins, including Munc13, Munc18, Complexin and Synaptotagmin, seems to stabilize the different states of the three-helical bundle suggesting that the acceptor complex could be regulated by these factors in the synapse. Hence, although generation of a stable acceptor complex is rate-limiting for ternary SNARE complex assembly in vitro, different factors might regulate this step in vivo and may cease to being rate-limiting (see partially assembled SNARE complexes).

Another interesting question is whether the intermediate acceptor complexes have been directly observed in native membranes in intact cells and whether they are always in Qabc composition. Indeed, there has been no direct evidence found, so far, for acceptor complexes in vivo and the interpretations from different studies are at times conflicting. For instance, association of the neuronal Syntaxin 1a and SNAP-25 is observed using livecell imaging or in SNARE-binding experiments on supported native membrane sheets (Lang et al., 2002, An and Almers, 2004). However, in the latter study, only the N-terminal SNARE region of SNAP-25 seems to be required for interaction. In an alternative study, a kinetic analysis performed using a cracked PC12 cell system suggests that SNAP-25 and Synaptobrevin associates prior to Syntaxin 1a binding (Chen et al., 2001). In other pathways, such as in retrograde ER-Golgi transport, a putative acceptor complex containing a Qabc composition has been suggested to exist. In this case, the complex formed of Ufe1p (Qa), Sec20p (Qb) and Use1p (Qc) tightly interacts with a multiprotein 
tethering complex, called Dsl1 (Kraynack et al., 2005).

In vitro denaturation experiments suggest that the late endosomal SNARE complex exhibits a similar hysteresis to the one of the synaptic SNARE complex, so that its assembly requires an intermediate (Fasshauer et al., 2002). Interestingly, the endosomal SNARE intermediate seems much less stable than the neuronal one and could not be clearly identified (Antonin et al., 2000, Fasshauer et al., 2002). Since the Qb- and QcSNARE motifs of the endosomal SNARE complex are contributed by two different proteins, Vti1b and Syntaxin 8, a different folding intermediate than the Qabc complex is possible to form (Fasshauer, 2003). Alternatively, more than one folding intermediates could exist.

Previously, it was observed that among all binary combinations of the neuronal SNAREs, only Syntaxin 1a and SNAP-25 form a stable complex (Fasshauer et al., 1997b). However, when the linker region between the two helices of SNAP-25 is removed, different stable complexes can form, e.g, ternary complexes containing Syntaxin 1a, Synaptobrevin and either the N-terminal or C-terminal SNARE regions of SNAP-25, and four-helical bundles composed of Syntaxin 1a and the N-terminal SNARE region of SNAP-25 (Fasshauer et al., 1997a, Fasshauer et al., 1998a, Misura et al., 2001a, Poirier et al., 1998). Moreover, the Syntaxin 1a SNARE motif is known to homo-oligomerize in solution (Misura et al., 2001b, Margittai et al., 2001). It remains to be seen whether such complexes formed between the neuronal SNAREs constitute alternative assembly pathways and whether they have biological relevance during fusion.

\section{Partially assembled SNARE complexes}

Electrophysiological studies propose existence of partially zippered SNARE complexes in neurons and neuroendocrine cells (Xu et al., 1998, Xu et al., 1999, Chen et al., 2001). It has been suggested that these metastable trans SNARE complexes could persist until arrival of action potential in synapses and the resulting $\mathrm{Ca}^{2+}$ influx. Partially zippered SNARE complexes are also inferred from the observation that a set of complexes appear to be susceptible to cleavage by certain clostridial neurotoxins at the C-terminus but not at the $\mathrm{N}$ terminus. Since in vitro the fully assembled SNARE complex is entirely resistant to proteolytic cleavage, it is thought that a partially zippered state exists where the $\mathrm{N}$ terminal portion of the SNARE complex is assembled and unsusceptible to toxin cleavage, 
while the C-terminus remains unstructured and therefore exposed to cleavage (Foran et al., 1994, Hua and Charlton, 1999). Evidence for such SNARE complexes has also been obtained in vitro by EPR measurements (Zhang et al., 2005). It is conceivable to imagine that SNARE zippering could stall before full assembly due to repulsive forces between the opposing membranes (Fasshauer, 2003). However, it remains to be answered what could affect the lifetime and later triggering of such complexes in vivo.

\subsubsection{Fusion}

Membrane bilayer fusion is believed to proceed through a series of ordered steps which involves merging of the proximal leaflets followed by merging of the distal leaflets of two initially opposed bilayers (Jahn and Grubmuller, 2002, Jahn and Scheller, 2006). According to the stalk hypothesis, the minimal events must involve the local deformation and partial dehydration of the membrane, the overcoming of the charge repulsion between the opposing membranes and formation of a lipid stalk, a local connection between the proximal leaflets of the two bilayers (Gingell and Ginsberg, 1978, Kozlov and Markin, 1983, Kozlovsky and Kozlov, 2002). The stalk then expands radially, so the idea, giving rise to a hemifusion diaphragm, a state where the proximal leaflets of the bilayers have fused and where the distal leaflets have formed a new bilayer separating the aqueous compartments. Disruption of the diaphragm leads to formation of a fusion pore, which provides an aqueous connection between the inside of the vesicle and the external environment (Chernomordik and Kozlov, 2008, Chernomordik et al., 2006).

How could SNAREs act as catalysts during the fusion event? SNAREs are thought to reside on "both" of the membranes undergoing fusion. SNARE zippering in this trans configuration starting from the N-terminus could then exert mechanical force on the membranes. For transducing the force on the membranes the linker region connecting the core complex to the transmembrane region of SNAREs is likely responsible. The linker region is thought to be rigid, so that straining the linkers could transmit energy onto membrane to distort the water-lipid boundary (Jahn et al., 2003). There is, indeed, experimental evidence supporting this model. When linker regions of SNAREs are extended by flexible residues, fusion of reconstituted proteoliposomes containing purified SNARE proteins is inhibited (Wang et al., 2001, McNew et al., 1999). An active role of the transmembrane region in mediating fusion is also plausible since fusion is inhibited when 


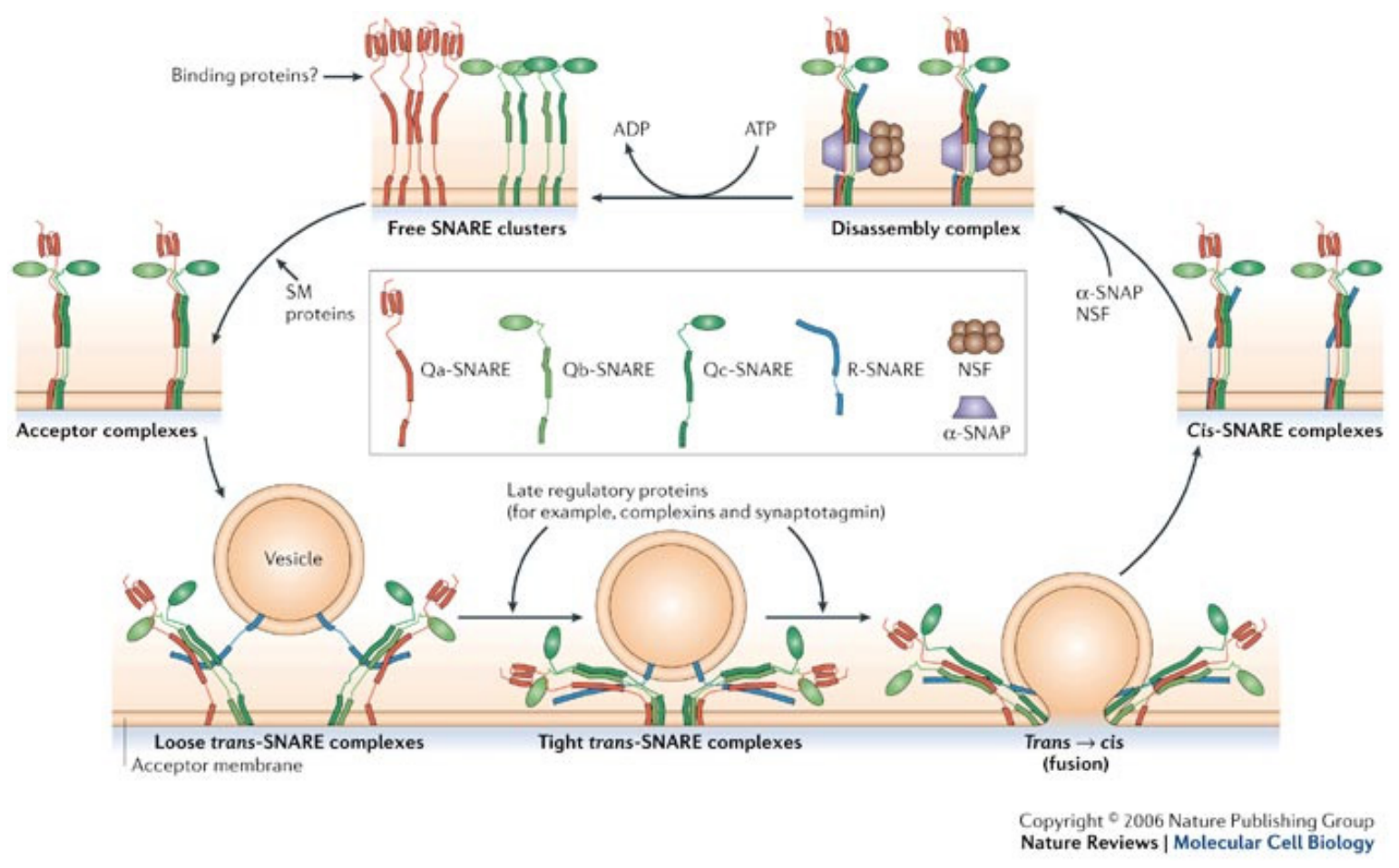

Figure 1.3: Conformational cycle of SNAREs during a vesicle fusion event

In the model system above, Q-SNAREs are on the target membrane, whereas the R-SNARE is on the vesicle. Free Q-SNAREs are organized in clusters on the membrane and they form acceptor complexes. SM (Sec1/ Munc18-like) proteins are thought to be involved in this stage, but they are proposed to function also in later stages, after the SNARE assembly (see section 1.3). N-terminal zippering of all four-SNAREs initially form a partially assembled trans-SNARE complex. Late regulatory proteins such as complexin and synaptotagmin are thought to help completing the zippering process to form the tight trans-SNARE complex. For detailed information about the late regulatory proteins, see review Sorensen, 2009. Formation of tight trans-SNARE complex is followed by opening of the aqueous fusion pore. Meanwhile, the strained trans-complex relaxes into a cis-configuration. The cis-complexes are disassembled by NSF and a-SNAPs in an ATP-dependent manner (Jahn and Scheller, 2006).

a SNARE transmembrane domain is replaced by a flexible lipid anchor (Grote et al., 2000a). A recent crystal structure demonstrates that the neuronal SNARE core bundle is helically extended into the plasma membrane. The continuity of the four-helical bundle in the linker region supports the idea that SNARE assembly is coupled to the initiation of membrane merger (Stein et al., 2009).

Although SNARE-mediated fusion mechanism described is conceivable, many details are still missing. For instance, it is still not exactly known how many SNARE complexes are needed for a single fusion event (Montecucco et al., 2005). In addition, the intermediate stages of fusion are primarily hypothetical and little experimental data is available. It has been shown that SNARE-mediated fusion in both native membranes and in proteoliposomes could be arrested at a hemifusion state (Xu et al., 2005, Reese et al., 2005, 
Giraudo et al., 2005). However, it is still unknown whether the hemifusion is a genuine intermediate for fusion or if it rather represents a dead-end product, as is possible for viral fusion proteins (Chernomordik and Kozlov, 2003). A current model for SNARE-mediated fusion and the conformational cycle of SNAREs at different stages are summarized in figure 1.3.

\subsubsection{Disassembly}

After fusion, the SNARE complex is localized on the fused membrane in a relaxed cis configuration. This low-energy configuration is thought to be inactive for further cycles of fusion. To recycle the SNAREs, the cis-complex is actively disassembled by a AAA+-

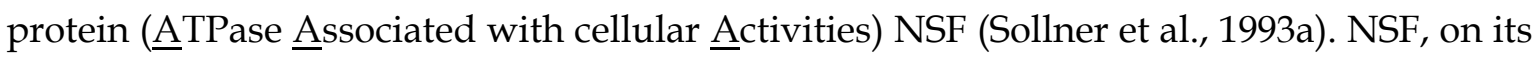
own, does not bind to SNARE complexes, and its cofactor a-SNAP (Soluble NSF Attachment Proteins) is thought to serve as an adaptor (Clary et al., 1990). Both NSF and SNAPs (including all three isoforms termed $\alpha-, \beta$ - and $\gamma^{-S N A P s)}$ are highly conserved and are thought to act on SNARE complexes at all transport steps (Whiteheart et al., 2001).

The domain organization of NSF consists of an N domain, followed by two homologous D domains (termed D1 and D2) that contain ATP-binding sites. The functional form of NSF is a hexameric ring conformation which is mediated by catalytically inactive D2 domains. The $\mathrm{N}$ domain is required for binding to the SNAP-SNARE complex and it undergoes large conformational changes during the catalytic action (Hanson and Whiteheart, 2005). The exact molecular mechanism of NSF-mediated SNARE complex disassembly is still elusive, but it has been proposed that the electrostatic interactions between a-SNAP and the acidic surface of the SNARE complex are important for NSF action (Marz et al., 2003). Interestingly, the NSF-SNAP system has been suggested to disassemble some of the off-pathway complexes previously discussed in section 1.2.3, and so could also act as a correction mechanism (Hanson et al., 1995, McMahon and Sudhof, 1995). Constitutive activity of this recycling system is important but not strictly required in vivo (Grote et al., 2000b, Sogaard et al., 1994, Littleton et al., 1998). 


\subsubsection{SNARE specificity}

In solution, the cytosolic portions of SNAREs assemble promiscuously into core complexes (Yang et al., 1999, Fasshauer et al., 1999). Consistent with the high structural conservation between the SNARE complexes, in some cases, SNAREs can substitute for each other, as long as they are within the same subfamily (Fasshauer, 2003, Jahn and Scheller, 2006). This suggests that non-cognate SNAREs would be able to unspecifically mediate fusion. However, a high degree of specificity has been observed in fusion of liposomes containing the yeast SNAREs (McNew et al., 2000, Parlati et al., 2000, Paumet et al., 2004). To explain this discrepancy, it has been claimed that the lipid bilayer induces a conformational change in the structure of SNARE motifs which prevents their association with non-cognate SNAREs. Nonetheless, there is no direct evidence for this explanation. Furthermore, more recent studies suggest that SNARE-mediated liposome fusion is also not strictly SNARE-specific. For instance, efficient liposome fusion was observed upon using endosomal and exocytic SNAREs together, although there is no physiological evidence for such fusion events (Brandhorst et al., 2006).

Considering that each eukaryotic cell is equipped with numerous SNAREs from each subfamily, how do vesicles fuse with each other specifically in vivo? Firstly, preferential distribution of individual SNAREs in distinct compartments is thought to provide some specificity. Although the precise sorting mechanism is unknown, many of the SNAREs reside predominantly in specific membranous compartments (Hong, 2005). For instance, Syntaxin 1, Syntaxin 2, Syntaxin 4, SNAP-25 and SNAP-23 are localized at the plasma membrane (Bennett et al., 1992, Bennett et al., 1993, Oyler et al., 1989, Wong et al., 1997), Synaptobrevin on exocytic vesicles (Baumert et al., 1989), and Syntaxin 5 in the Golgi apparatus (Dascher et al., 1994). In contrast to that, several other SNAREs, particularly the endosomal ones have a more widespread distribution. Thus, possibly not only SNAREs, but also further factors ensure specificity of intracellular vesicle fusion events. The conserved RabGTPase family is certainly thought to be instrumental for fusion specificity since they activate tethering complexes. In addition, tethering factors might add an additional layer of specificity since they provide the initial link between the vesicle and the target membrane (Brocker et al., 2010). Finally, a tight regulation in the formation of acceptor complexes might provide specific assembly of SNARE complexes, thus might influence fusion specificity (Hong, 2005, Jahn and Scheller, 2006, Pfeffer and Aivazian, 2004). 


\subsection{The SM protein family}

\subsubsection{Essential role of SM proteins in vesicle fusion}

SM (Sec1/ Munc18-like) proteins are central and indispensible factors of the intracellular trafficking machinery (Toonen and Verhage, 2003). Their loss-of-function results in severe defects in vesicular transport and fusion, which often leads to a lethal phenotype (see Table 1.1). Known crystal structures of distantly related members reveal that the overall fold of SM proteins is highly conserved between different organisms and at different vesicular trafficking steps (Bracher et al., 2000, Bracher and Weissenhorn, 2002, Misura et al., 2000, Hu et al., 2007, Burkhardt et al., 2011). The SM protein structure consists of three domains (domain 1-3), which form an arch-shape with a large central cavity.

The discovery of SM proteins dates back to the early 1970's. The first SM genes to be discovered were Unc-18 in Caenorhabditis elegans and Sec1 in Saccharomyces cerevisiae, which were independently identified in genetic screens (Brenner, 1974, Novick and Schekman, 1979, Novick et al., 1980). Since a temperature-sensitive mutation Sec1-1 in yeast caused excessive accumulation of vesicles near the plasma membrane, Sec1 was thought to be an element of the secretory pathway (Novick and Schekman, 1979). Unc-18 mutant worms, on the other hand, were characterized by uncoordinated movement and accumulation of acetylcholine (Brenner, 1974, Gengyo-Ando et al., 1993). The association of Unc-18 with neurotransmission was first established by identification of Munc18a, the mammalian homologue of Unc-18, interacting stably with Syntaxin 1a in the rat brain homogenates (Hata et al., 1993). Later non-neuronal homologues of Munc18a were also identified, Munc18b and Munc18c, both required for exocytosis (Garcia et al., 1994, Hata and Sudhof, 1995, Tellam et al., 1995). At around the same time, the gene encoding the SM protein Sly1p was discovered in yeast and was found to be essential in fusion events between the Endoplasmic reticulum and the Golgi apparatus (Dascher et al., 1991, Ossig et al., 1991). Later, Munc18a has been realised to be indispensible for neurotransmission, since deletion of Munc18a was lethal in mice blocking neurosecretion entirely in the prenatal synapses (Verhage et al., 2000). With the identification of more members of the SM protein family in different organisms and at different trafficking steps, SM proteins have slowly become thought to be the essential components of the fusion machinery, rather than being primarily involved in regulation of fusion. At present, the SM protein family includes seven members in vertebrates (Munc18a, Munc18b, Munc18c, Vps33a, 


\section{Table 1.1: The SM proteins that are discussed in this study}

The table is adapted from Toonen and Verhage, 2003. See Toonen and Verhage, 2003 for related literature. N.D. : not determined.

*Related literature: Burkhardt et al., 2011.

\begin{tabular}{|c|c|c|c|c|c|}
\hline Species & $\begin{array}{l}\text { SM } \\
\text { protein }\end{array}$ & $\begin{array}{l}\text { Subcellular } \\
\text { Localization }\end{array}$ & $\begin{array}{l}\text { Syntaxin } \\
\text { interactions }\end{array}$ & $\begin{array}{l}\text { Null-mutant } \\
\text { phenotype }\end{array}$ & $\begin{array}{l}\text { Proposed } \\
\text { function }\end{array}$ \\
\hline \multirow{4}{*}{$\begin{array}{l}\text { Saccharomyces } \\
\text { cerevisiae }\end{array}$} & Sec1p & $\begin{array}{l}\mathrm{CM} \text { at sites of } \\
\text { exocytosis }\end{array}$ & $\begin{array}{l}\text { Sso1p, } \\
\text { Sso2p }\end{array}$ & Lethal & Exocytosis \\
\hline & Sly1p & ER/ Golgi membrane & $\begin{array}{l}\text { Sed5p, } \\
\text { Ufe1p }\end{array}$ & Lethal & ER to Golgi \\
\hline & Vps33p & Vacuolar membrane & Vam3p & Viable & $\begin{array}{l}\text { Endosome } \\
\text { to vacuole }\end{array}$ \\
\hline & Vps45p & $\begin{array}{l}\text { TGN/ early \& } \\
\text { late endosome }\end{array}$ & $\begin{array}{l}\text { Tg12p, } \\
\text { Pep12p }\end{array}$ & Viable & $\begin{array}{l}\text { Golgi-vacuole, } \\
\text { Golgi-late } \\
\text { endosome }\end{array}$ \\
\hline $\begin{array}{l}\text { Drosophila } \\
\text { melanogaster }\end{array}$ & ROP & $\begin{array}{l}\text { Cytosol \& } \\
\text { membrane associated }\end{array}$ & Syntaxin & $\begin{array}{l}\text { Lethal, } \\
\text { embryo }\end{array}$ & $\begin{array}{l}\text { General and } \\
\text { synaptic } \\
\text { exocytosis }\end{array}$ \\
\hline $\begin{array}{l}\text { Caenorhabditis } \\
\text { elegans }\end{array}$ & Unc-18 & N.D. & Unc-64 & Uncoordinated & $\begin{array}{l}\text { Synaptic } \\
\text { exocytosis }\end{array}$ \\
\hline \multirow{5}{*}{$\begin{array}{l}\text { Mus } \\
\text { musculus }\end{array}$} & Munc18a & $\begin{array}{l}\text { Cytosol \& } \\
\text { membrane associated }\end{array}$ & Syntaxin 1, 2, 3 & Lethal & $\begin{array}{l}\text { Synaptic } \\
\text { exocytosis }\end{array}$ \\
\hline & Munc18b & $\begin{array}{l}\text { Cytosol \& } \\
\text { membrane associated }\end{array}$ & Syntaxin 1, 2, 3 & N.D. & $\begin{array}{l}\text { Apical trafficking } \\
\text { in epithelial cells }\end{array}$ \\
\hline & Munc18c & $\begin{array}{l}\text { Cytosol \& } \\
\text { membrane associated; } \\
\text { insulin dependent }\end{array}$ & Syntaxin 2,4 & Lethal & $\begin{array}{l}\text { GLUT4 vesicle } \\
\text { fusion with } \\
\text { membrane }\end{array}$ \\
\hline & $\mathrm{mVps} 45$ & $\begin{array}{l}\text { Golgi/ endosomal } \\
\text { membrane }\end{array}$ & Syntaxin 16 & N.D. & N.D. \\
\hline & mSly1 & N.D. & Syntaxin 5,18 & N.D. & ER to Golgi \\
\hline $\begin{array}{l}\text { Monosiga* } \\
\text { brevicollis }\end{array}$ & Munc18 & Apical pole & Syntaxin 1 & N.D. & N.D. \\
\hline
\end{tabular}

Vps33b, Vps45, Sly1) and four in yeast (Sec1p, Vps33p, Vps45p, Sly1p) (see Table 1.1).

Exemplified by the stable interaction between Munc18a and Syntaxin 1a, the primary binding partners of SM proteins are Qa-SNAREs (syntaxins) (reviewed in Toonen and Verhage, 2003). Initial biochemical studies revealed that the complex of Munc18a/Syntaxin 1a blocks formation of the core SNARE complex with SNAP-25 and 
Synaptobrevin (Pevsner et al., 1994, Yang et al., 2000). Later, the crystal structure of Munc18a/Syntaxin 1a complex (figure 1.4) demonstrated that Munc18a tightly grasps a closed conformation of the syntaxin, and showing that it is the structural configuration of Munc18a-bound Syntaxin 1a which impedes the accessibility for its partner SNAREs (Misura et al., 2000). Two years after structural characterization of Munc18a / Syntaxin 1a complex, interactions between Sly1 and its cognate syntaxins were investigated (Yamaguchi et al., 2002). It was shown that Sly1 binds to a short, evolutionary conserved N-terminal peptide of Sed5p and Ufe1p in yeast and of syntaxins 5 and 18 in vertebrates. Shortly after, Sly1p was crystallized in complex with the short N-terminal peptide of Sed5p, showing that the N-peptide binds to domain 1 of Sly1p, on the opposite site of the Munc18a/Syntaxin 1a binding site (Bracher and Weissenhorn, 2002). Since sequence conservation suggested that Sly1p homologues as well as Vps45p paralogues bind their cognate syntaxins in the same way (Bracher and Weissenhorn, 2002), this interaction mode was defined as a potentially general mechanism for SM proteins. In the same year, the yeast and vertebrate Vps45 was shown to bind Tlg2p and Syntaxin18, using the Npeptide motif of the syntaxins in support of this notion (Dulubova et al., 2002). In this way, a classical concept has arised slowly, stating that SM proteins generally interact with the N-peptide motifs of syntaxins and Munc18a binding to Syntaxin 1a is an exception.

At present, a consensus has been reached about SM proteins that they have an essential role in fusion and interact with syntaxins specifically. However, there are contradicting findings regarding their precise mode of action and at which steps of the fusion process they affect (reviewed in Toonen and Verhage, 2003, Toonen and Verhage, 2007, Sudhof and Rothman, 2009, Carr and Rizo, 2010, Sorensen, 2009). In the following sections, the interplay between SM proteins and SNAREs and the functional implications will be briefly reviewed. General features of some of the SM proteins, which will be discussed in the next sections, are summarized in table 1.1.

\section{Interaction with syntaxins}

In general, interactions of SM proteins with syntaxins occur with high specificity and nanomolar affinity. According to the classical concept, SM proteins interact with their cognate syntaxins in two distinct modes. The first mode (termed mode 1) involves binding of the SM protein to a closed conformation of the syntaxin. This interaction mode 
was first observed between Syntaxin 1a and the synaptic SM protein Munc18a (Figure 1.4 A) and has been proposed to block SNARE assembly since it renders Syntaxin 1a inaccessible for its partner SNAREs (Pevsner et al., 1994, Dulubova et al., 1999, Misura et al., 2000, Yang et al., 2000). In the second and apparently a more general interaction mode (termed mode 2), the SM protein binds only to a short $\mathrm{N}$-terminal peptide of the syntaxin independent of its conformation at its C-terminus (Figure 1.4 B). Examples where this has been shown include yeast and vertebrate Sly1, binding to Sed5p/ Syntaxin 5 and to Ufe1p/ Syntaxin 18; yeast and vertebrate Vps45 binding to Tlg2p/ Syntaxin 16; and vertebrate Munc18c binding to Syntaxin 4 (Bracher and Weissenhorn, 2002, Yamaguchi et al., 2002, Dulubova et al., 2002, Hu et al., 2007, Arac et al., 2005, Latham et al., 2006). This interaction mode is thought to facilitate SNARE assembly rather than inhibiting it (Toonen and Verhage, 2003, Sudhof and Rothman, 2009, Carr and Rizo, 2010), however, a well-defined mechanism accounting for this interpretation have not been brought by yet.

Although the structural properties of both SM proteins and syntaxins seem to be highly conserved, it has been puzzling to understand why two distinct modes of interaction between SM proteins and syntaxins have evolved. In addition, mode 1 binding of Munc18a brings about discrepancies for the functioning of the SM protein. As previously discussed, biochemical and structural evidence suggests that Munc18a acts as a negative regulator for SNARE complex formation since it stabilizes the closed conformation of Syntaxin 1a (Pevsner et al., 1994, Dulubova et al., 1999, Misura et al., 2000, Yang et al., 2000). Paradoxically, the loss of Munc18a blocks neurosecretion in vivo, rather than causing constitutive secretion as what would be expected for an exclusively mode 1 mechanism (Voets et al., 2001, Gulyas-Kovacs et al., 2007, Verhage et al., 2000). To reconcile the apparently contradictory observations, it has been suggested that this mode is not universal, but is rather a specialization of neuronal secretion (Rizo and Rosenmund, 2008, Sudhof and Rothman, 2009, Shen et al., 2007, Deak et al., 2009) .

More recent findings have helped to resolve some of these discrepancies. First, a reexamination of the original Munc18a/Syntaxin 1a X-ray diffraction data (Misura et al., 2000) has shown that the N-terminal peptide motif of Syntaxin 1a serves as a second binding site for Munc18a (Burkhardt et al., 2008) (Figure 1.4 A). A detailed thermodynamic analysis revealed that the peptide motif binds Munc18a much weaker than the closed conformation, yet still enhances the overall affinity of the Munc18a/ Syntaxin 1a interaction (Burkhardt et al., 2008). Interestingly, binding of the N-peptide to 


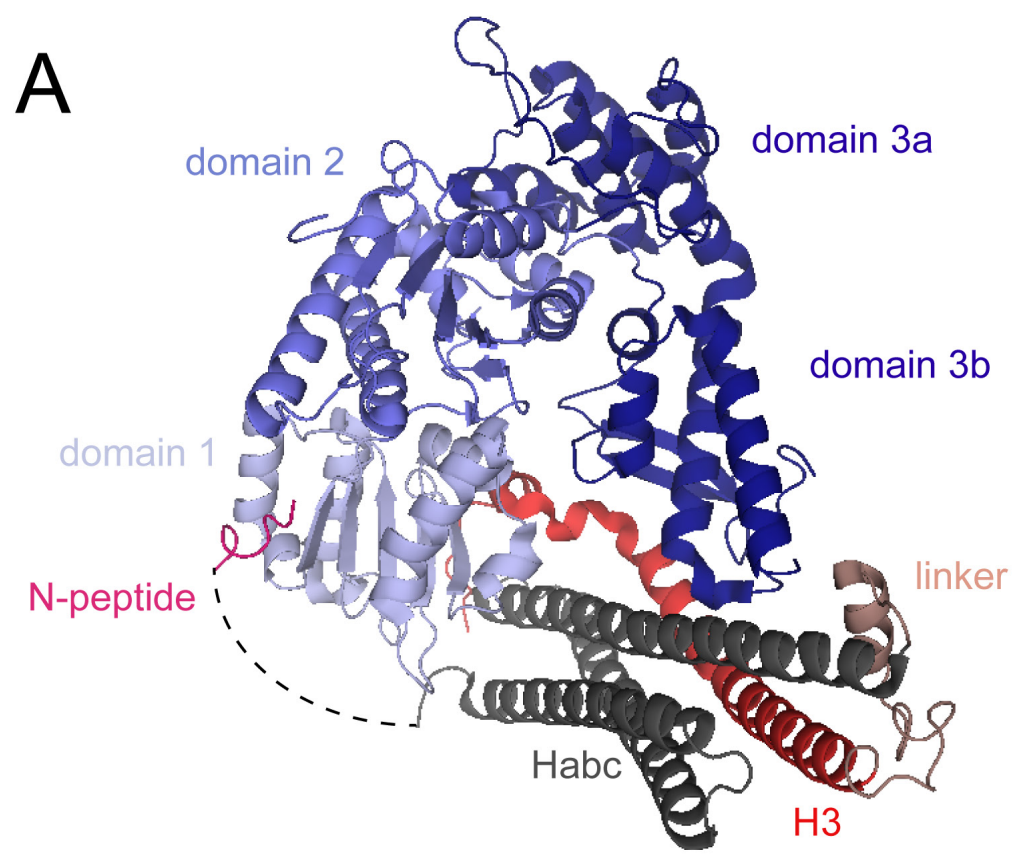

Neuronal Munc18a/ Syntaxin 1a

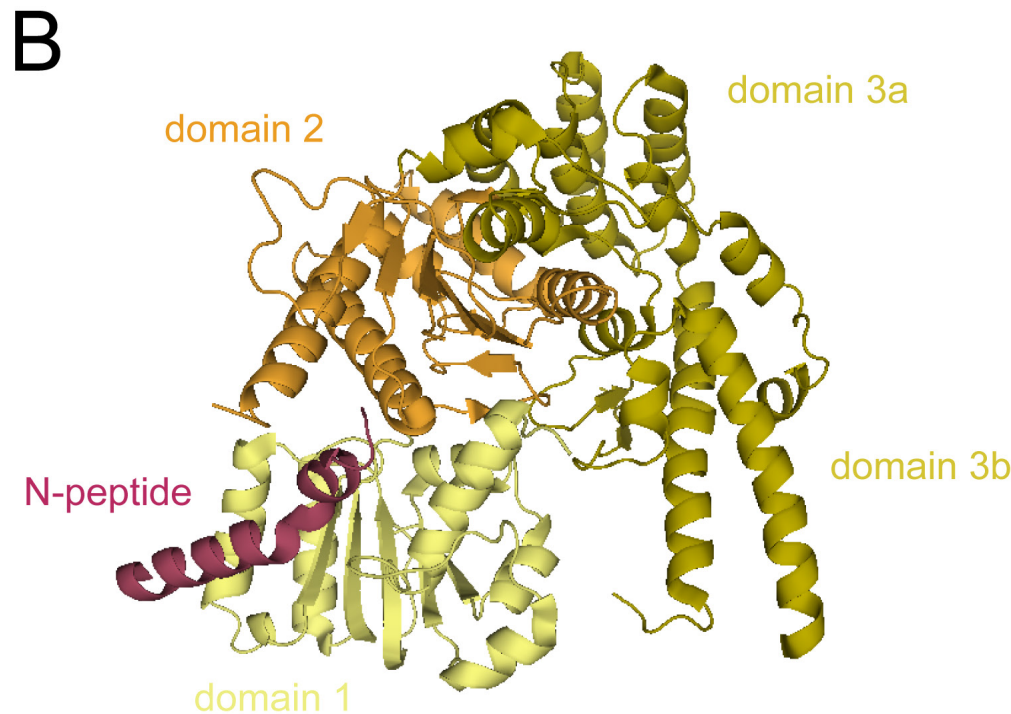

Yeast Sly1p/ Sed5p

\section{Figure 1.4: Crystal structures of Munc18a (A) and of Sly1p (B) in complex with their cognate syntaxins}

A. Munc18a domains 1, 2 and 3 are colored in different shades of blue. Note that domain 3 is subdivided into domains $3 a$ and $3 b$ (Misura et al., 2000). The first 10 residues of the Syntaxin $1 \mathrm{a}$ N-peptide that have been resolved in the re-refined crystal structure is shown in magenta (Burkhardt et al., 2008). The dashed lines show residues 10-26 of Syntaxin 1a which are not visible in electron density maps. The Habc domain and the SNARE (H3) motif of Syntaxin 1a are colored in grey and red, respectively. B. Sed5p (1-21) interacts with the N-terminal domain of Sly1p, at a site opposite to the central cavity where neuronal Syntaxin 1a binds to Munc18a. Domain architecture of Sly1p is displayed in yellow for domain 1, orange for domain 2 and green for domain 3. Sed5p is represented in purple. 
the outer surface of Munc18a seems to be necessary for Munc18a to control the accessibility of Syntaxin 1a for its SNARE partners. When the N-peptide is removed, the block of Munc18a is relieved, allowing Syntaxin 1a to form a SNARE complex while still bound to Munc18a (Burkhardt et al., 2008). Although it remains unclear how this switch occurs, it appears that the two binding sites between Munc18a and Syntaxin 1a act together during SNARE complex formation. In the same study, Burkhardt and co-workers also showed that the remainder of the Syntaxin 16, but not solely the peptide motif is involved in Vps45/ Syntaxin 16 interaction (Burkhardt et al., 2008), suggesting that the ability for an SM protein to bind to a syntaxin using both mode 1 and mode 2 is a general property.

Additional biochemical studies on different SM protein/ Syntaxin pairs have gone on to support this idea. In recent years, it has been revealed that three other exocytic SM proteins make use of two binding sites when interacting with their cognate syntaxins: vertebrate Munc18b (with Syntaxin 3) and Munc18c (with Syntaxin 4), and Unc-18 from Caenorhabditis elegans (with Unc-64) (Peng et al., 2010, D'Andrea-Merrins et al., 2007, Johnson et al., 2009). In addition, among the non-exocytic SM proteins, not only the vertebrate Vps45 (Burkhardt et al., 2008), but also its yeast homologue (i.e., Vps45p) has been proposed to use the two modes when binding to syntaxin (i.e. Tlg2p) (Furgason et al., 2009). As shown in a very recent study, Munc18/ Syntaxin 1 from the unicellular choanoflagellate Monosiga brevicollis, the sole secretory SM protein/ syntaxin pair in this species, uses both binding sites, the N-peptide and the closed conformation. Furthermore, the biochemical analysis and the crystal structure of those proteins strongly suggest that their mode of interaction is strikingly similar to that of Munc18a/ Syntaxin 1a (Burkhardt et al., 2011). Thus, it is very likely that mode 1 binding is not a unique specialization of neuronal secretion but instead is part of a highly conserved interaction mechanism between SM proteins and syntaxins which involves both the N-peptide and the closed conformation of a syntaxin.

\section{Interactions with SNARE complexes}

As explained in the previous section, the binary interaction of an SM protein with a syntaxin involves two well-defined binding sites. However, at least one SM protein, the yeast exocytic Sec1p, does not appear to interact with a monomeric syntaxin. It has been 
suggested that, Sec1p, instead, predominantly interacts with an assembled SNARE complex containing one of its cognate syntaxins, Sso1p or Sso2p (Togneri et al., 2006, Carr et al., 1999, Scott et al., 2004). Although the interaction mode between Sec1p and the fourhelix SNARE bundle has not been biochemically characterized yet, it has been suggested that this interaction facilitates membrane fusion in vivo and also SNARE-mediated liposome fusion in vitro (Grote et al., 2000b, Scott et al., 2004).

Other SM proteins, including yeast Sly1p and Vps45p, and vertebrate Munc18a, Munc18b and Munc18c, have also been shown to interact with preassembled SNARE complexes in vitro (Peng and Gallwitz, 2002, Carpp et al., 2006, Dulubova et al., 2007, Burkhardt et al., 2008, Xu et al., 2010, Peng et al., 2010, Latham et al., 2006). Interaction of Munc18a with the neuronal SNARE complex has been the major focus of several studies in recent years, since this binding mode is proposed to assist SNARE complex and promote fusion (Rizo and Rosenmund, 2008, Sudhof and Rothman, 2009, Carr and Rizo, 2010). Biophysical studies demonstrate that Munc18a/ SNARE complex interaction involves the N-terminal Habc domain of Syntaxin 1a and possibly also the four-helical bundle of the SNARE complex (Dulubova et al., 2007, Xu et al., 2010). Munc18a binds to the entire SNARE complex with submicromolar affinity (Dulubova et al., 2007, Burkhardt et al., 2008, Xu et al., 2010), whereas it binds to the core region of the SNARE complex very weakly, with low micromolar affinity $(\sim 6 \mu \mathrm{M})(\mathrm{Xu}$ et al., 2010). It has been proposed that the inner cavity of Munc18a "clasps" the four-helical SNARE bundle during fusion, yet the precise interaction surface of the proteins has not been mapped; thus, further work is still needed to validate the SNARE complex binding mode of Munc18a (Rizo and Rosenmund, 2008, Sudhof and Rothman, 2009). The proposed models for functioning of this binding mode will be discussed in section 1.3.2.

\section{Interactions via multiprotein complexes}

The yeast SM protein Vps33p is involved in Golgi-to-vacuole transport and is part of a multiprotein complex termed HOPS (ㅂomotypic Fusion and vacuole Protein $\underline{\text { Sorting) }}$ (Seals et al., 2000). HOPS is a hexameric effector complex and is together with Rab GTPase Ypt7p required in the tethering of vacuoles (Mayer and Wickner, 1997, Stroupe et al., 2009). Although several lines of evidence suggest functional coupling of SM proteins with Rabs and tethering complexes, the HOPS complex represents a unique case, since it 
demonstrates a physical connection between them (Dascher et al., 1991, Cao et al., 1998). HOPS complexes have been shown to bind to trans-SNARE complexes (Collins and Wickner, 2007), as well as to monomeric SNAREs Vam3p (Qa) (Price et al., 2000, Dulubova et al., 2001) and Vam7p (Qc) (Stroupe et al., 2006). Although the molecular mechanism is unknown, the HOPS complex is proposed to promote trans-SNARE pairing between fusing vacuole membranes (Mima et al., 2008, Stroupe et al., 2009).

\subsubsection{Functional studies on SM proteins}

Do SM proteins confer specificity to SNARE-mediated trafficking?

As mentioned in section 1.3.1, SM proteins interact with syntaxins in a highly specific manner in vitro and in vivo (Toonen and Verhage, 2003). This is different from the interactions between syntaxins and other SNARE proteins, since the cytosolic portions of SNAREs can assemble promiscuously into core complexes in vitro (Yang et al., 1999, Fasshauer et al., 1999). It is plausible, therefore, that, SM proteins could be one of the factors conferring the needed specificity for SNARE complex assembly in vivo. Different studies are consistent with this idea. For instance, Sly1p-bound Sed5p (Qa) is unable to form nonphysiological SNARE complexes in vitro (Peng and Gallwitz, 2002). The HOPS complex containing the yeast Vps33p also seems to have a similar proofreading role during SNARE assembly. HOPS, interestingly, suppresses vacuole fusion when transSNARE complexes are formed without the N-terminal domain of Vamp7p (Qc), or if complexes are not of the 3Q:1R composition (Starai et al., 2008).

In addition to their role in regulating specific intracellular trafficking steps, SM proteins also seem to have cell-type specific roles. For instance, Munc18a functions during action potential-triggered exocytosis in neurons and neuroendocrine cells (Hata et al., 1993, Verhage et al., 2000), whereas Munc18b is specialized for constitutive exocytosis (Riento et al., 2000), and Munc18c modulates exocytosis in a variety of nonneuronal cell types, e.g., the insulin-dependent GLUT4 (Glucose transporter 4)-containing vesicles in muscle and fat cells (Thurmond et al., 1998, Thurmond et al., 2000). As mentioned previously, neurosecretion is fully blocked in neurons in the absence of Munc18a, despite the presence of Munc18b and Munc18c in those cells (Verhage et al., 2000). Similarly, Munc18b cannot compensate for the loss of Munc18c in skeletal muscle for regulated 
translocation of GLUT4 onto the plasma membrane (Khan et al., 2001). In another example, the cholinergic defects in C. elegans Unc-18 null mutants can be overcome by exogeneous expression of murine Munc18a, but not murine Munc18b (Gengyo-Ando et al., 1996). Thus, SM proteins are likely to be one of the factors contributing to vesicle fusion specificity in the secretory pathway.

\section{Do SM proteins act as chaperones for SNAREs?}

Several studies suggest that at least some SM proteins might act like chaperones for their cognate syntaxins. As an example, in yeast cells lacking Vps45p, the syntaxin analogue Tlg2 $p$ levels are diminished to undetectable levels by rapid proteasomal degradation (Bryant and James, 2001). In another study, it has been shown that yeast Sly1p protects one of its cognate syntaxins, Ufe1p, against ER-associated degradation (ERAD). Different than this, Sly1p does not influence the stability of Sed5p, its second binding partner (Braun and Jentsch, 2007). Unc-18 null worms have a $\sim 50 \%$ reduction of the syntaxin analogue Unc-64 (Weimer et al., 2003), and it has been recently shown that Unc-64 in the absence of Unc-18 mainly accumulates in the ER (McEwen and Kaplan, 2008). Neuronal Munc18a seems also to have a chaperone role, since Munc18a knockout mice have $~ 70 \%$ decreased levels of Syntaxin 1a (Toonen et al., 2005). Moreover, Munc18a has been suggested to prevent ectopic interactions of Syntaxin 1a with other SNAREs during its transport to the plasma membrane (Medine et al., 2007).

Could a chaperone-like function account for the severe trafficking defects, which are observed in the absence of SM proteins? Although tempting, this is an unlikely possibility. In yeast cells lacking Vps45p, Tlg2p can be stabilized by abolition of proteasomal activity. Tlg2p can be correctly targeted to its correct intracellular location in those cells, yet is unable to bind its cognate partner SNAREs (Bryant and James, 2001). In Unc-18 null worms, overexpression of Unc-64 does not rescue secretion and behavioral defects (Weimer et al., 2003). Furthermore, mutations disrupting Unc-18 interaction with the Npeptide of Unc-64 restore Unc-64 trafficking, but not secretion-related defects (McEwen and Kaplan, 2008). In Munc18a knockout mice, residual Syntaxin 1a is still correctly targeted to the plasma membrane (Toonen et al., 2005) and rescue of Munc18a null chromaffin cells with Munc18a mutants restores Syntaxin 1a levels, but docking and/or fusion phenotypes remain (Gulyas-Kovacs et al., 2007). Thus, other aspects of SM proteins 
apart from their chaperone-like functions seem to play a critical role for intracellular trafficking.

\section{At which stages of trafficking do SM proteins function?}

In the absence of SM proteins, transport vesicles can reach to the vicinity of their target compartments, yet, they cannot complete fusion. Therefore, SM proteins are thought to function during the last steps of a trafficking event, i.e., during vesicle tethering/ docking or during SNARE complex formation or after the SNARE complex has been formed. Several studies are consistent with a role of SM proteins at promoting vesicle docking to its target compartment. For instance, in Vps45p null yeast cells or in mouse adipocytes expressing Munc18c mutants, transport vesicles accumulate adjacent to vacuoles or the plasma membrane, respectively (Cowles et al., 1994, Thurmond et al., 2000). Dramatic vesicle docking defects are also observed in the neurons of C. elegans Unc-18 mutants (Weimer et al., 2003), and neurons of D. melanogaster ROP mutants (see table 1.1) (Schulze et al., 1994). However, yeast Sly1p mutants block fusion without affecting vesicle attachment to the target, suggesting a function downstream of docking (Cao et al., 1998). Hence, it is plausible that certain SM proteins might have specialized jobs in the trafficking routes that they regulate.

Specific roles of the neuronal Munc18a taking part during sequential events of exocytosis have been uncovered, since exocytosis of large dense core vesicle (LDCV) in chromaffin cells can be conveniently monitored at high temporal resolution by electrophysiological methods (Neher, 1998, Voets et al., 1999, Voets, 2000, Xu et al., 1999). Based on this approach, Munc18a is thought to participate in two sequential steps of exocytosis (Gulyas-Kovacs et al., 2007). The first step involves vesicle docking, in good agreement with the severe docking defects observed in chromaffin cells in the absence of Munc18a (Voets et al., 2001). In this step, Munc18a is proposed to induce vesicle docking, dependent on its ability to bind to the closed conformation of Syntaxin 1a. A double mutation in Munc18a (D34N/M38V), which is thought to disturb binding to the closed conformation perturbs this step. This early step is believed to be independent of SNAREpairing, as it was demonstrated not to be affected by deletion of vesicle-SNAREs or SNAP-25 in chromaffin cells (Borisovska et al., 2005, Sorensen et al., 2003). Thus, Munc18a is thought to be involved in the docking step in combination with other factors such as 
vesicle-associated Doc2 (Dascher et al., 1994), plasma-membrane associated Mint (Okamoto and Sudhof, 1997) or Munc13 (Sassa et al., 1999) or perhaps with a Rab protein (Dascher et al., 1991).

Since the second step is not affected by Munc18a (D34N/M38V) mutation, it is believed to be independent from the closed conformation interaction and, Munc18a is thought to interact with the SNARE machinery in a different mode. In this step, Munc18a is suggested to stimulate a process called "vesicle priming", in which a docked vesicle gains release competence by sequential events. It has been proposed that during priming, Munc18a could promote SNARE assembly by its interaction with folding intermediates. However, no in vitro studies have managed to reproduce the proposed effects of Munc18a on the assembly of neuronal SNAREs. An alternative view holds that Munc18a stimulates a later step in exocytosis after zipping of the SNARE complex during fusion pore opening (Fisher et al., 2001), although this finding has also been questioned by some (GulyasKovacs et al., 2007).

In summary, biological evidence suggests that an SM protein might act on multiple steps during a vesicle fusion event. The SM protein could function upstream or downstream of SNARE complex formation or both; however, conflicting evidence from different studies makes it challenging to come up with a general role. In addition, SM proteins might have specialized jobs in different trafficking steps, and thus it might not be possible to impose a general role for the SM proteins. Putative functions of different SM proteins during or after SNARE assembly will be discussed in the next sections.

\section{How do SM proteins function during SNARE complex formation?}

As mentioned in section 1.3.1, the high affinity binding partners of most SM proteins are syntaxins. Thus, one of the major tasks of SM proteins might be to modulate SNARE assembly through their interaction with syntaxins. Due to their positive regulatory role in secretion, and having discarded an alternative chaperone-like function as the direct cause, it seems highly plausible that SM proteins are required to orchestrate SNARE assembly. Several studies have examined this primarily using in vitro pull-down assays in which SNARE complex formation is followed over time, but no consensus on the action of SM proteins on SNARE complex formation has been found. For instance, Munc18c and Vps45p but not Sly1p have been reported to stimulate SNARE assembly (Struthers et al., 
2009, Latham et al., 2006, Peng and Gallwitz, 2002). Further studies, possibly with more quantitative readouts, seem to be necessary in order to reproduce the putative stimulatory effect of SM proteins during the SNARE assembly process.

As previously discussed, structural and biochemical data suggests that Munc18a can inhibit neuronal SNARE assembly (Pevsner et al., 1994, Yang et al., 2000, Misura et al., 2000). It is now known that Munc18a binds Syntaxin 1a using two binding modes via the $\mathrm{N}$-peptide and the closed conformation of Syntaxin 1a (Burkhardt et al., 2008). The binding status of Munc18a to these distinct sites seems to affect the conformation of the Munc18a/Syntaxin 1a complex and thus the regulation of SNARE assembly. As mentioned before, Burkhardt and co-workers, have shown that when the N-peptide is removed, the block on SNARE assembly is also relieved. Removal of the N-peptide is presumed to cause a conformational change in the Munc18a/Syntaxin 1a complex, since both binary and ternary SNARE complexes are able to form, suggesting Munc18a controls the accessibility of Syntaxin 1a to its SNARE partners. Therefore, the interplay between the two binding sites of an SM protein/syntaxin pair could be a way to switch the regulation of SNARE assembly. In principle, this switch could also occur in vivo possibly with the help of accessory factors and could constitute a gating mechanism to allow SNARE assembly after an initial block. However, more direct evidence for this hypothesis has yet to be presented.

\section{Do SM proteins stimulate fusion by interacting with assembled SNARE complexes?}

As mentioned above, at least one SM protein, yeast exocytic Sec1p, is thought to act on assembled SNARE complexes, a hypothesis supported by both in vivo and in vitro evidence (Togneri et al., 2006, Carr et al., 1999, Scott et al., 2004). Other SM proteins such as Sly1p, Vps45p, Munc18b and Munc18c have been also reported to bind to assembled SNARE complexes; however, the functional relevance of this binding mode has not been followed up for those SM proteins (Peng and Gallwitz, 2002, Carpp et al., 2006, Peng et al., 2010, Latham et al., 2006). In an in vitro assay consisting of fusion of proteoliposomes containing neuronal SNAREs, Munc18a has been shown to enhance fusion (Shen et al., 2007). Since Munc18a does not seem to stimulate the assembly kinetics of SNAREs (Pevsner et al., 1994, Yang et al., 2000, Burkhardt et al., 2008), the observed enhancement has been suggested to occur after SNARE complex formation. After the discovery that 
Munc18a can bind to assembled SNARE complexes, this notion has gained further support (Dulubova et al., 2007, Xu et al., 2010). Two models have been put forward to describe how the stimulation could occur on assembled SNARE complexes. In the first model, grappling of the trans-SNARE complex by the SM protein prevents diffusion of the SNAREs to the intermembrane space, where they could hinder fusion (Rizo et al., 2006). In the second model, trans-SNARE complex assembly results in stabilization of a hemifusion intermediate. Clasping of the SM protein over the SNARE bundle is proposed to transiently destabilize the hemi-fusion intermediate and induce the formation of a fusion pore (Carr and Rizo, 2010).

Binding of Munc18a to the assembled SNARE complex as inferred from liposome fusion studies resolves some of the discrepancies between previous in vitro and in vivo studies. Still, contradictory results from different physiological studies question this notion (Fisher et al., 2001, Gulyas-Kovacs et al., 2007). Structural and biochemical observations are also unfavorable towards the stimulatory SNARE complex binding mode. For instance, binding of Munc18a to the core SNARE bundle is very weak, which is inconsistent with a tight "clasping" mechanism during fusion (Xu et al., 2010, Burkhardt et al., 2008). In addition, the liposome fusion study that demonstrates the stimulatory action of Munc18a during fusion cannot detect exactly in which step the stimulation occurs (Shen et al., 2007). In this assay system, the fusion of proteoliposomes containing SNAREs is measured via a FRET-based readout, which reflects lipid-mixing between the two populations of liposomes. Thus, the readout solely monitors the last step of the fusion process and any specific regulation in preceding steps is indistinguishable. Hence, it is not possible to pinpoint at what stage of SNARE assembly the stimulatory effect of Munc18a occurs.

Overall, it is plausible to imagine SM proteins acting on assembled SNARE complexes since this binding mode seems to be used by several SM proteins. However, there is no biochemical and physiological evidence providing direct proof so far.

What is the role of the N-peptide for functioning of SM proteins?

As explained in section 1.3.2, several syntaxins are thought to use mode 2 (N-peptide) binding for their interactions with SM proteins. Thus, the short N-peptide motif of syntaxins could be important for the cellular functioning of SM proteins. Concurrent with this notion, transfection of Vero cells with the N-peptide motif of Syntaxin 5 severely 
disrupts the Golgi complex, possibly by interfering with the endogeneous N-peptide interaction with Sly1. Conversely, mutant forms of the N-peptide, which show no interaction with Sly1 have no effect on Golgi morphology (Yamaguchi et al., 2002). Two other recent studies also point out the physiological importance of the Syntaxin N-peptide motifs (McEwen and Kaplan, 2008, Johnson et al., 2009). In both studies, it has been reported that mutations in Unc-18, which are predicted to disrupt the binding of Unc-64 $\mathrm{N}$-peptide, result in neuromuscular trafficking defects. These in vivo observations also correlate well with the findings obtained by in vitro assays using Munc18a and Syntaxin 1a. Direct abolition of Munc18a interaction with the N-peptide of Syntaxin 1a removes the inhibitory action of Munc18a on the SNARE assembly, as well as the stimulatory action observed in in vitro liposome fusion assays (Shen et al., 2007, Burkhardt et al., 2008).

In contrast to the aforementioned studies, disruption of the N-peptide binding does not lead to discernible phenotypes in other systems. When N-peptide interactions of two yeast SM proteins, Sly1p and Vps45p, were disrupted by point mutations, no trafficking defects were observed (Peng and Gallwitz, 2004, Carpp et al., 2006). Hence, it is still unclear how exactly the N-peptide modulates the functioning of an SM protein. Possible scenarios will be discussed in later sections.

\subsection{ER-to-Golgi transport in yeast and its regulation by Sly1p}

\subsubsection{Anterograde transport between the ER and Golgi in yeast}

Owing to the availability of straightforward genetic tools, baker's yeast has been in the front row since late 1970's for studying the molecular machineries involved in vesicular trafficking pathways. At present, 25 SNAREs are thought to form the overall repertoire of SNARE-mediated fusion machinery in yeast (Burri and Lithgow, 2004, Kienle et al., 2009). For most transport steps, distinct units of four interacting SNARE motifs have been assigned although with uncertainties since some SNAREs are involved in more than one step (Hong, 2005, Jahn and Scheller, 2006, Burri and Lithgow, 2004, Pelham, 1999, Kienle et al., 2009) (Figure 1.5).

Genetic, biochemical and morphological evidence suggests that yeast SNAREs Sed5p $(\mathrm{Qa}), \operatorname{Bos} 1 \mathrm{p}(\mathrm{Qb}), \operatorname{Bet} 1 \mathrm{p}(\mathrm{Qc})$ and Sec22p (R) function as a single unit for anterograde 


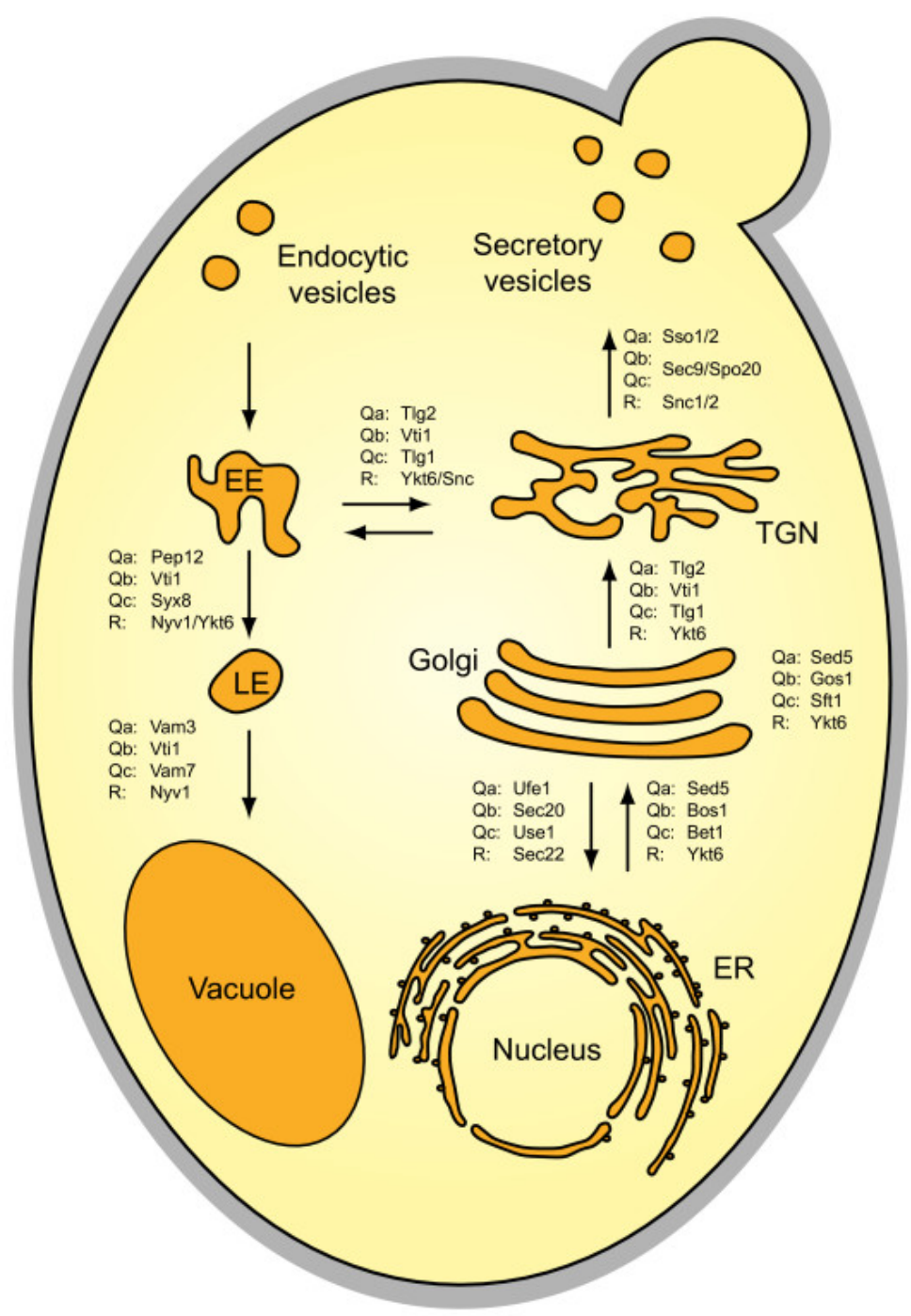

\section{Figure 1.5: Tentative assignment of the involved sets of SNAREs in S. cerevisiae}

Functional SNARE units involved in distinct trafficking pathways are allocated based on SNARE classification according to HMM models (see Kienle et al, 2009). It needs to be mentioned, however, that the assignment of the R-SNAREs in different trafficking steps is still debated. Note that, according to the prevalent view, the RSNARE for ER-to-Golgi transport is Sec22p, which is functionally redundant with Ykt6p.

transport between ER and Golgi (Kaiser and Schekman, 1990, Newman et al., 1990, Hardwick and Pelham, 1992, Sogaard et al., 1994). In contrast to Qa-, Qb- and QcSNAREs, the R-SNARE Sec22p is not essential and its function can be substituted with another R-SNARE, Ykt6p (Liu and Barlowe, 2002). Interestingly, the two R-SNAREs belong to different SNARE subclasses (Kienle et al., 2009) and their SNARE-binding properties differ, which will be discussed in the subsequent sections. Like several other Qa-SNAREs, the domain organization of Sed5p consists of a short N-terminal peptide followed by the Habc domain, and the SNARE motif (Yamaguchi et al., 2002). Biochemical 
data has suggested that individual Sed5p might adopt a closed conformation (Mossessova et al., 2003); however, direct evidence is missing so far. The Qb- and Qc- SNAREs, Bos1p and Bet1p, have also N-terminal domains, yet their structural properties are not precisely known. Nonetheless, limited proteolysis experiments indicate that the N-terminal domain of Bos1p might be structured (Mossessova et al., 2003). Furthermore, according to a protein structure prediction algorithm (Lupas et al., 1991), the N-terminal part of Bos1p has nearly a $100 \%$ propensity of being involved in a coiled coil interaction, suggesting that this region might bind to an unknown protein component (Sacher et al., 1997). The two RSNAREs implicated to be involved in the ER-to-Golgi trafficking, Sec22p and Ykt6p, have $\mathrm{N}$-terminal profilin-like folds, so-called longin domains (Figure 1.2 B). Based on structural evidence, each of these R-SNAREs can adopt a closed conformation in which the longin domain is folded back on the SNARE motif (Mancias and Goldberg, 2007, Tochio et al., 2001).

The assembly mechanism of the aforementioned ER-Golgi SNAREs has not been studied so far. Nevertheless, in vitro fusion experiments with SNAREs reconstituted into synthetic liposomes have suggested that the SNARE assembly and thus the fusion occurs only when the Qa-, Qb- and R-SNAREs are on one population of liposomes and the Qc-SNARE is on the other (Parlati et al., 2000). The interpretation of this finding was that the QabRSNAREs and the Qc-SNARE must have been topologically restricted by design to function on separate membrane compartments. However, other interpretations are also possible which will be discussed in this study. It needs to be mentioned, additionally, that the proteoliposome fusion assays are somewhat contradictory to the in vitro budding and fusion experiments that reconstitute transport between the ER and Golgi. According to these experiments, Bet1p $(\mathrm{Qc})$ and $\operatorname{Bos} 1 \mathrm{p}(\mathrm{Qb})$ are both required on the ER-derived vesicle (Cao and Barlowe, 2000). Making things more complicated, the subcellular localization of the ER-to-Golgi SNAREs is also not precisely defined in vivo, except for Sed5p (Qa), which is located preferentially on the cis side of the Golgi (Hardwick and Pelham, 1992, Banfield et al., 1994).

Sed5p (Qa) is one of the yeast SNAREs that is involved in more than one trafficking steps. In addition to the ER-to-Golgi transport, it has been found to function also in retrograde transport within the Golgi apparatus (Figure 1.5), by forming a SNARE complex with Gos1p (Qb), Sft1p (Qc) and Ykt6p (R) (Parlati et al., 2002). Interestingly, Sed5p seems to be able to form promiscuous binary interactions with each of its 7 partner SNAREs in vitro 
(Tsui and Banfield, 2000). Thus, an interesting question which arises is what kind of mechanism provides the specificity in the Golgi to distinguish vesicles arriving from different donor compartments. According to one study, Sed5p containing ternary and quaternary SNARE complexes form specifically in solution, such that Sed5p containing intermediates can select for the subsequent binding partner (Tsui et al., 2001). Putting in other words, this study suggests that different composition of binary and ternary complexes might induce different folding intermediates which are ready to accept distinct SNARE partners. Thus, an auto-regulatory mechanism of SNAREs during their assembly can provide some specificity to vesicle fusion.

Although SNARE-mediated fusion specificity might hold true, for the initial contact of a vesicle on its acceptor compartment, other factors such as tethering complexes, Rab family GTPases and coat proteins seem to play major role (see Figure 1.1). For instance, the initial capture of ER-derived transport vesicles on the Golgi seems to be SNARE-independent and rather requires a RabGTPase Ypt1p and a long coiled-coil tether Uso1p (Cao et al., 1998). In addition to Uso1p, a large oligomeric tethering complex called TRAPPI, which is stably anchored on the Golgi membrane, seems to be the landmark for attracting ERderived vesicles (Sacher et al., 1998, Sacher et al., 2000, Sacher et al., 2001). Surprisingly, TRAPPI tethers ER-derived vesicles via interaction with Sec23p, an inner layer component of the vesicle coat (Cai et al., 2007). Thus, ER-derived vesicles might not be entirely shed their coats before reaching to Golgi and thus, the coat proteins on the vesicles might provide additional specificity during tethering. It has been suggested that tethers might merely enhance the SNARE encounter or might catalytically rearrange SNARE complexes to facilitate fusion, yet the mechanistic details of this process is still largely unknown (see reviews Sztul and Lupashin, 2009, Spang, 2009, Whyte and Munro, 2002).

\subsubsection{Role of Sly1p in the ER-to-Golgi transport step}

The SM protein that is involved in the ER-Golgi trafficking pathway in yeast is Sly1p (see Table 1.1). Sly1p was first identified in a genetic screen since a single amino acid substitution (E532K) of a dominant mutant, termed Sly1-20 (Suppressor of Loss of $\underline{Y} p t 1$ function), was able to bypass the deletion of the Rab protein Ypt1p (Dascher et al., 1991). This was also the first indication for the functional coupling between SM proteins, SNAREs and Rabs. Sly1p seems to be an essential protein for cell viability since its 
depletion is lethal in yeast (Ossig et al., 1991). In addition, through a targeted siRNA screen, its vertebrate analogue Sly1 has been recently found to be required for constitutive secretion in mammalian cells (Gordon et al., 2010).

Sly1p is thought to be involved in the bi-directional transport between the ER and Golgi (Ossig et al., 1991, Li et al., 2005). Its high affinity binding partners are the syntaxins, Sed5p and Ufe1p, which function in the anterograde and in the retrograde trafficking pathways respectively (see Figure 1.5) (Grabowski and Gallwitz, 1997, Kosodo et al., 1998, Yamaguchi et al., 2002, Bracher and Weissenhorn, 2002). Sly1p has been shown to use solely the mode 2 binding (N-peptide) for its interaction with the monomeric syntaxins. The crystal structure of Sly1p in complex with the N-peptide of Sed5p has already been determined at a resolution of $3.0 \AA$ (Bracher and Weissenhorn, 2002). This has been the second structure of an SM family protein after the neuronal Munc18a (Misura et al., 2000).

Aside from its closely similar overall fold, Sly1p has sequence insertions compared to Munc18a at residues corresponding to a-helix 20. a-20 together with a-21 have been suggested to act as a lid controlling a function of Sly1p, since these helices partially shield the external surface formed by $\alpha-13$ and $\alpha-14$, one of the most conserved surface regions among the Sly1p homologues (Bracher and Weissenhorn, 2002) (Figure 1.6). Interestingly, the single amino acid substitution of the Sly1-20 mutant (E532K) lies on the a-20 as well, suggesting that the lid might be permanently open in the mutant and thereby might bypass a Ypt1p guarded regulatory step (Bracher and Weissenhorn, 2002). Supporting this notion, it has been shown in a later study that the deletion of YPT1 can be suppressed by a Sly1p mutant, in which the entire a-20 is removed ( $\mathrm{Li}$ et al., 2007). There has been no direct interaction detected between Sly1p and Rabs so far, thus the Rab-related function of Sly1p might be dependent on a physical interaction with a yet undefined effector. In addition, a-20 seems to be highly conserved in fungi, but is absent in other eukaryotes, suggesting that Sly1p might have a lineage-specific functioning in a Rab-dependent tethering step of vesicles ( $\mathrm{Li}$ et al., 2007).

The crystal structure of the Sly1p/ Sed5p complex demonstrates that Sed5p N-peptide (residues 1-21) interacts predominantly with domain 1 of Sly1p by forming two a-helices separated by residue 5 . The helical structure of bound Sed5p is most likely induced by Sly1p since this region is unstructured in solution (Bracher and Weissenhorn, 2002, Yamaguchi et al., 2002). The N-terminal residues of Sed5p (residues 1-10), constituting a 

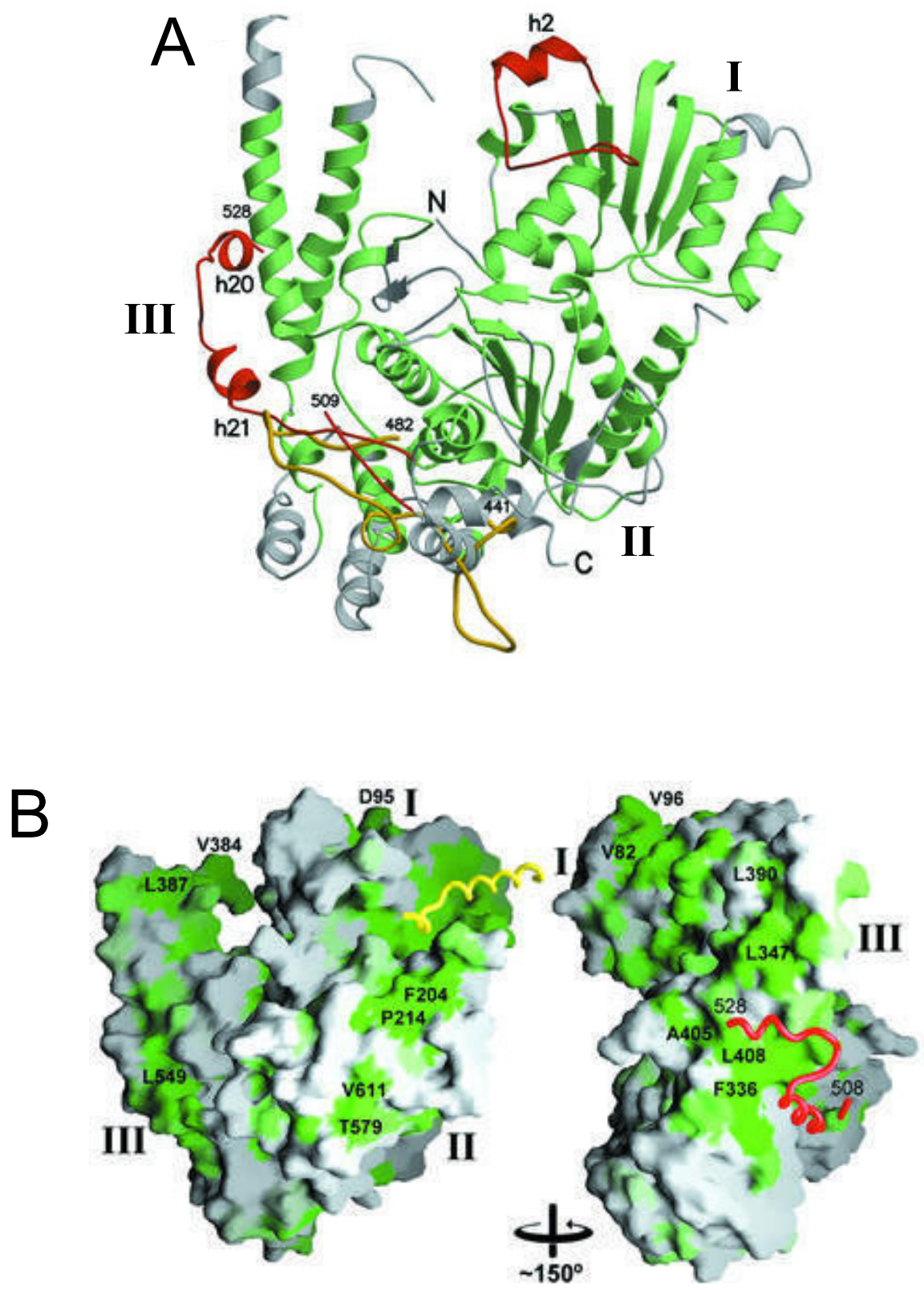

Figure 1.6: Comparison of Sly1p with Munc18a and the Sly1p homologues

A. Ribbon presentation of Sly1p, superimposed with neuronal Munc18a. Regions superimposing with an r.m.s.d. $<3.5 \AA$ are shown in green, those > $3.5 \AA$ are in grey. Sequence insertions in Sly1p resulting in independent secondary structure elements are in red. The loop region between a-21 and a-22 in Munc18a is shown in yellow for comparison. B. Surface conservation among the Sly1p homologues is plotted to the surface of Sly1p using a scale from green (identical) to white (no conservation). Coils denote the Sed5p Npeptide (yellow) and the helices a-20 and a-21 (red). To point out some conserved residues, the image is also shown re-oriented after a $\sim 150^{\circ}$ rotation around the vertical axis. Figure is adapted from Bracher and Weissenhorn, 2002. 
conserved sequence signature among Sed5p homologues (Yamaguchi et al., 2002), bind Sly1p through mainly hydrophobic interactions. The most critical residue for these interactions seems to be Phe10, which is accommodated in a highly conserved hydrophobic pocket by Sly1p. Its mutation into Alanine has been reported to abrogate Sly1p-Sed5p interactions (Yamaguchi et al., 2002), suggesting a nucleation role for Phe10 in adopting the helical binding mode (Bracher and Weissenhorn, 2002).

Several lines of evidence suggest that Sly1p plays a positive regulatory role for the SNARE assembly in the ER-to-Golgi transport. For instance, in vitro SNARE-binding assays have demonstrated that Sly1p prevents Sed5p from forming presumably nonphysiological SNARE complexes (Peng and Gallwitz, 2002). In vivo studies interfering with the interaction between vertebrate Sly1p (Sly1) and Sed5p (Syntaxin 5) orthologues have been shown to induce Golgi fragmentation and/or inhibition of ER-to-Golgi transport (Dulubova et al., 2003, Yamaguchi et al., 2002, Williams et al., 2004). Thus, Npeptide interaction of Sly1p-Sed5p seems to be biologically important and required for the integrity of the secretory pathway. Opposite to this notion, however, a study by Peng \& Gallwitz has suggested that the high-affinity interaction between Sly1p and Sed5p is dispensable and not relevant for proper function in vivo (Peng and Gallwitz, 2004). In this study, various mutant forms of Sly1p and Sed5p, which abolish their N-peptide interaction, have been found fully functional in vivo in the absence of their wild-type counterparts. The strains that have only the mutant copies of either Sly1p or Sed5p have shown no defects in cell growth, the vesicular protein transport or the localization of the SM protein. Since in the same study Sly1p was shown to bind the SNARE motifs of nonsyntaxin SNAREs, Bos1p (Qb) and Bet1p (Qc), the interpretation was that Sly1p and/or associated proteins possibly have a "bridging" role between the components of the SNARE complex. This might hold true, however, it excludes the possibility that additional regions on Sed5p can be involved in Sly1p binding and can be sufficient for the SM protein to bind Sed5p and act on the SNARE assembly. Possible interpretations of this study will be discussed in the further sections. 


\subsection{Aim of the work}

Sec1/ Munc18 (SM) proteins are important regulators of intracellular membrane fusion. SM proteins interact with Qa-SNAREs (syntaxins) with high specificity and high affinity. So far, two binding sites have been characterized between SM proteins and syntaxins. Neuronal Munc18a interacts with a closed conformation of Syntaxin 1a, whereas in other examples, a short $\mathrm{N}$-terminal peptide of the syntaxin binds to the SM protein (reviewed in Toonen and Verhage, 2003). However, recent findings suggest that all SM proteins might exert a regulatory role, acting on both the $\mathrm{N}$-peptide motifs and the closed conformation of syntaxins (Burkhardt et al., 2008).

Yeast Sly1p, the SM protein of ER-to-Golgi trafficking step, is thought to interact only with the N-peptide motif of the syntaxin Sed5p (Yamaguchi et al., 2002, Bracher and Weissenhorn, 2002, Peng and Gallwitz, 2002). However, a putative second binding site has not been investigated yet. In light of the proposed common binding model, one of the major goals of this project was to understand whether Sly1p/Sed5p interaction involves two binding sites in analogy to Munc18a/Syntaxin 1a complex. Initially I aimed to examine whether individual Sed5p can adopt a closed conformation. Afterwards, I characterised the Sly1p/Sed5p interaction using biochemical and biophysical tools in order to understand whether a second binding site, involving a closed conformation of Sed5p can bind Sly1p.

The SNAREs functioning in the ER-to-Golgi transport step are Sed5p (Qa), Bos1p (Qb), Bet1p (Qc) and Sec22p (R) (Kaiser and Schekman, 1990, Newman et al., 1990, Hardwick and Pelham, 1992, Sogaard et al., 1994). In previous studies, based on qualitative readouts, Sly1p has been reported to have no effect in the assembly kinetics of the ER-Golgi SNAREs, but rather to have a proofreading role (Peng and Gallwitz, 2002). Another important objective of this project was to re-examine the action of Sly1p on the SNARE complex formation, based on quantitative read-outs. Since the assembly mechanism of ER-Golgi SNAREs was not studied before, I initially developed the tools to understand the assembly pathways of the SNAREs and the dynamics of their interactions. In the next step, I investigated the regulatory role of Sly1p on the ER-Golgi SNAREs and whether the regulation involves a collaborative action of the two binding sites on Sly1p. 


\section{Chapter 2}

\section{Materials \& Methods}

\subsection{Materials}

\subsubsection{Chemicals}

All chemicals used in the study were purchased from BioRad, Boehringer, Merck, Roth, Serva and Sigma. Thiol-reactive probes (Oregon Green 488 Iodoacetamide and Texas Red Maleimide) were purchased from Molcular Probes/ Invitrogen.

\subsubsection{Enzymes, kits, bacterial strains and vectors}

Restriction endonucleases, Phusion high-fidelity DNA polymerase and T4-DNA-Ligase used during cloning were purchased from New England Biolabs. Lysozyme and DnaseI used for protein purification were purchased from AppliChem. Thrombin (Bovine) from MP Biomedicals was used for the cleavage of polyHis- or GST-tags. The kits used for cloning were from Macherey-Nagel. E. coli XL1-Blue (Stratagene) strain was used for cloning and E. coli BL21 (DE3) (Novagen) strain was used for protein expression. All DNA constructs generated in the study were cloned into pET28a vectors (Novagen).

\subsubsection{DNA constructs}

DNA constructs were PCR amplified from S. cerevisiae cDNA. Expression constructs were cloned into pET28a plasmid vectors using standard methodology. For nitroxide (MTSL) 
or fluorescent labeling, single amino acid residues were substituted with cysteine residues via site-directed mutagenesis. A two-step PCR strategy was used to generate the cysteine variants. Since native Ykt6p has two cysteines, the cysteine residue at position 197 was substituted with a Serine residue to enable fluorescent labeling at a single position. The list of all DNA fragments used in this study is shown in table 2.1. Some of the constructs were kindly provided by Dr. Renwang Peng (Peng and Gallwitz, 2002, Peng and Gallwitz, 2004).

\subsubsection{Peptides}

The polypeptides corresponding to the N-peptide domains of Sed5p and Ufe1p were chemically synthesized by Biosyntan $\mathrm{GmbH}$ (Berlin). The purity of the peptides was

Table 2.1: DNA constructs used in the study

\begin{tabular}{|c|c|c|c|c|}
\hline GENE & FRAGMENT & MUTATION & PLASMID & SOURCE \\
\hline \multirow[t]{2}{*}{ Sly1p } & $1-666$ & - & pGEX-2T & Peng \\
\hline & $1-260$ & - & pGEX-2T & Peng \\
\hline \multirow{7}{*}{ Sed5p } & \multirow{3}{*}{$1-320$} & - & pET28a & Demircioglu \\
\hline & & F10A & pET28a & Demircioglu \\
\hline & & $\begin{array}{l}\text { K22C, E254C, S278C, } \\
\text { E282C, D291C, S302C }\end{array}$ & pET28a & Demircioglu \\
\hline & \multirow[t]{2}{*}{$1-210$} & - & pET28a & Demircioglu \\
\hline & & $\mathrm{K} 22 \mathrm{C}$ & pET28a & Demircioglu \\
\hline & $21-324$ & - & pET28a & Peng \\
\hline & $211-320$ & - & pET28a & Demircioglu \\
\hline \multirow[t]{2}{*}{ Bos1p } & \multirow[t]{2}{*}{$151-221$} & - & pET28a & Demircioglu \\
\hline & & E181C & pET28a & Demircioglu \\
\hline \multirow[t]{2}{*}{ Bet1p } & \multirow[t]{2}{*}{$1-118$} & - & pET19b & Peng \\
\hline & & G85C & pET28a & Demircioglu \\
\hline \multirow{5}{*}{ Sec $22 p$} & \multirow[t]{2}{*}{$126-186$} & - & pET28a & Peng \\
\hline & & D131C, K180C & pET28a & Demircioglu \\
\hline & $143-186$ & - & pET28a & Demircioglu \\
\hline & $146-186$ & - & pET28a & Demircioglu \\
\hline & $150-186$ & - & pET28a & Demircioglu \\
\hline Ykt6p & $131-200$ & C197S & pET28a & Demircioglu \\
\hline
\end{tabular}


analyzed by HPLC and mass spectrometry. F10A and K22C mutants of the Sed5p Npeptide were also chemically synthesized. The amino acid sequence of the peptides are shown below:

\section{Sed5p 1-21: MNIKDRTSEFQQSVLSYKKRN}

Sed5p 1-21 F10A: MNIKDRTSEAQQSVLSYKKRN

Sed5p 1-22 K22C: MNIKDRTSEFQQSVLSYKKRNC

Ufe1p 1-21: MMSDLTPIFRKYVAVIDDARN

\subsubsection{Gene synthesis}

In order to replace the entire linker domain of Sed5p with a flexible peptide linker, a Sed5p construct denoted "Sed5p-linked" was designed. In order to determine the boundaries of the linker fragment to be substituted, the C-terminal boundary of the Habc domain and the N-terminal boundary of the H3 motif needed to be defined. The Cterminal boundary of the Hc helix was not precisely defined by the previous NMR studies (Yamaguchi et al., 2002), yet sequence alignment of Sed5p from several fungi species shows conservation up to residue 182 (see Appendix A.1). The N-terminal boundary of the SNARE motif is also not precisely defined (residue 258 according to SNARE database: http:/ / bioinformatics.mpibpc.mpg.de/snare/, Kloepper et al, 2007), however, the flexible linker segment up to residue 232 was extended. Since residue 232 is a Proline, it is possible that it could be the first residue of a helix due to its structural rigidity. Consequently, the Sed5p fragment spanning residues 182-232 was replaced with a flexible peptide. The flexible peptide was designed to contain 5 GGSGGS repeats and the DNA construct corresponding to the "Sed5p-linked" was chemically synthesized by the firm Mr. Gene (Invitrogen).

\subsection{Methods}

\subsubsection{Protein expression and purification}

Prior to recombinant expression of a protein, the corresponding DNA construct was first 
electroporated into E. coli BL21 (DE3) electro-competent cells. For this, 50-100 $\mu$ l of bacteria was thawed on ice and mixed with about $10 \mathrm{ng}$ of DNA. After incubation on ice for 15 minutes, the cells were transferred to pre-cooled $0.2 \mathrm{~cm}$ electroporation cuvettes (BioRad). Electroporation was performed at $1.8 \mathrm{kV}, 200 \Omega$ and $25 \mathrm{mF}$ using a Gene Pulser (BioRad). Before plating on LB agar plates, cells were mixed with $1 \mathrm{ml}$ Luria Bertani (LB) medium $(1 \%(\mathrm{w} / \mathrm{v})$ tryptone, $0.5 \%(\mathrm{w} / \mathrm{v})$ yeast extract, $1 \%(\mathrm{w} / \mathrm{v}) \mathrm{NaCl}, \mathrm{pH}: 7.0)$ containing $100 \mathrm{mM}$ glucose and were grown in a shaker for 1 hour at $37^{\circ} \mathrm{C}$.

For expression, one of the transformed colonies was picked and grown in LB medium (containing either $100 \mu \mathrm{g} / \mathrm{ml}$ of Ampicillin or $30 \mu \mathrm{g} / \mathrm{ml}$ of Kanamycin depending on the plasmid vector) overnight at $37^{\circ} \mathrm{C}$. Expression was done in 2L Erlenmeyer flasks containing $800 \mathrm{ml}$ Terrific Broth (TB) medium (1.2\% (w/v) tryptone, 2.4\% (w/v) yeast extract, $0.4 \%$ (w/v) glycerin), $10 \%$ (v/v) TB salt $\left(0.17 \mathrm{M} \mathrm{KH}_{2} \mathrm{PO}_{4}, 0.72 \mathrm{M} \mathrm{K}_{2} \mathrm{HPO}_{4}\right)$ and the relevant antibiotic. Each flask was inoculated with 10-15 $\mathrm{ml}$ overnight culture and cells were grown in a shaking incubator for 3 hours at $37^{\circ} \mathrm{C}$. Protein expression was induced by addition of $0.25 \mathrm{mM}$ IPTG into media, when cells reached to late-log phase $\left(\mathrm{O}^{\mathrm{D}} \mathrm{D}_{600} \approx\right.$ 0.8-1). Apart from Sly1p (1-666), all proteins were expressed at $37^{\circ} \mathrm{C}$ for 3 hours. Sly1p expression was optimized at $20^{\circ} \mathrm{C}$ overnight. After protein expression, cells were harvested by centrifugation at $4000 \mathrm{rpm}$ for $15 \mathrm{~min}$ using a Beckman J6-MI. The cell pellet was resuspended either in $\mathrm{Ni}^{2+}$-washing buffer $(20 \mathrm{mM}$ Tris $\mathrm{pH}$ 7.4, $500 \mathrm{mM} \mathrm{NaCl}, 8 \mathrm{mM}$ imidazole) or in GST-washing buffer (20 mM Tris pH 7.4, $150 \mathrm{mM} \mathrm{NaCl}, 1 \mathrm{mM}$ EDTA, 1 mM DTT) depending on the construct. The cell pellet was stored at $-20^{\circ} \mathrm{C}$ for future use.

For affinity purification, the cell suspension was thawed at $37^{\circ} \mathrm{C}$. Then, it was mixed with PMSF and $1 \mathrm{mg} / \mathrm{ml}$ lysozyme and incubated at room temperature for $20 \mathrm{~min}$. This was followed by addition of $5 \mathrm{mg}$ DnaseI, $1 \mathrm{mM} \mathrm{MgCl}$, and 1\% (v/v) Triton X-100. Cells were incubated at room temperature for $15 \mathrm{~min}$. In order to break the cells, the suspension was sonicated by $4 \times 30 \mathrm{sec}$ pulses with a Branson Sonifier. Immediately after, 1mM PMSF was added and the lysate was incubated at room temperature for $10 \mathrm{~min}$ again. For SNARE proteins, 6M urea was added after this step. Bacterial debris was removed by centrifugation at $12500 \mathrm{rpm}$ for 1 hour. The supernatant containing the protein of interest was incubated either with $\mathrm{Ni}^{2+}$-NTA-agarose or Glutathione sepharose beads for about 2 hours at $4^{\circ} \mathrm{C}$. 
$\mathrm{Ni}^{2+}$-NTA-beads were washed with $\sim 250 \mathrm{ml} \mathrm{Ni}{ }^{2+}$-washing buffer $(20 \mathrm{mM}$ Tris pH 7.4, 500 $\mathrm{mM} \mathrm{NaCl}, 8 \mathrm{mM}$ imidazole) and the His ${ }_{6}$-tagged proteins were eluted with elution buffer (2x12 ml, Ni²+-washing buffer containing $400 \mathrm{mM}$ imidazole). Except for Sed5p (1-320), no urea was included in the washing and the elution buffers of SNAREs to provide rapid renaturation. Sed5p (1-320) was washed and eluted in the presence of $2 \mathrm{M}$ urea. In all cases, Thrombin was added to the eluates and dialysed overnight at $4^{\circ} \mathrm{C}$. The dialysis buffer contained $20 \mathrm{mM}$ Tris $\mathrm{pH}$ 7.4, $1 \mathrm{mM}$ EDTA, $1 \mathrm{mM}$ DTT and $\mathrm{NaCl}$. The $\mathrm{NaCl}$ concentration was $20 \mathrm{mM}$ for Sed5p (1-320) and about $100 \mathrm{mM}$ for all other SNAREs. The dialysis buffer of Sed5p (1-320) contained 2M urea in addition.

For Sly1p (1-666) and (1-260), Glutathione sepharose beads were used. The beads were washed with $\sim 200 \mathrm{ml}$ washing buffer $(20 \mathrm{mM}$ Tris $\mathrm{pH}$ 7.4, $150 \mathrm{mM} \mathrm{NaCl}, 1 \mathrm{mM}$ EDTA, 1 mM DTT), with $\sim 8 \mathrm{ml}$ of buffer left incubating with the beads. Thrombin was added to mixture and the GST tags of the proteins were cleaved on the beads by overnight incubation at $4^{\circ} \mathrm{C}$. The eluate was collected in the following day.

Except for Sly1p constructs, all proteins were further purified by ion-exchange chromatography. Depending on the $\mathrm{pI}$ of the proteins, either MonoQ HR 10/10 or MonoS HR 10/10 columns (Pharmacia Biotech) were used. Proteins were eluted by using a linear $\mathrm{NaCl}$ gradient in buffer A (20 mM Tris pH 7.4, 1mM EDTA) and buffer B (buffer A containing $1 \mathrm{M} \mathrm{NaCl}$ ) by using an ÄKTA explorer. For Cysteine variants, $1 \mathrm{mM}$ DTT was added into each buffer. For Sed5p (1-320), 2M urea was included in the buffers and after elution, the urea was removed by dialysis (dialysis buffer: $20 \mathrm{mM}$ Tris pH:7.4, $200 \mathrm{mM}$ $\mathrm{NaCl}, 1 \mathrm{mM}$ EDTA). Sly1p (1-666) and (1-260) were further purified on HiLoad 26/60 Superdex 200 columns in a buffer containing $20 \mathrm{mM}$ Tris pH:7.4, $200 \mathrm{mM} \mathrm{NaCl}, 1 \mathrm{mM}$ EDTA and 1mM DTT. All proteins were eluted in $3 \mathrm{ml}$ fractions. The fractions were analysed by SDS-PAGE gel electrophoresis and the peak fractions were pooled and were either used immediately or were snap frozen for storage at $-80^{\circ} \mathrm{C}$. Sly1p constructs were frozen and stored in the presence of $10 \%(\mathrm{v} / \mathrm{v})$ glycerol.

Protein complexes used in this study were assembled by mixing equimolar amounts of the individual components in the presence of $500 \mathrm{mM} \mathrm{NaCl}$. Mixtures containing only the SNARE motif of Sed5p (QaH3) were incubated overnight at $4^{\circ} \mathrm{C}$, whereas those containing the full-length Sed5p (Qafull) were incubated for 3-4 days due to slow formation of the complexes. Excess $\mathrm{NaCl}$ was removed via dialysis and the monomers 
were separated from the complexes by using ion-exchange chromatography (MonoQ HR $5 / 5)$. The protein complexes were eluted by using a linear $\mathrm{NaCl}$ gradient in the buffer solutions described above.

\subsubsection{Isothermal Titration Calorimetry (ITC)}

All ITC experiments were done using a VP-ITC instrument (MicroCal, GE Healthcare). The ITC system consists of two twin coin-shaped cells, i.e., the sample and the reference cell, mounted in an adiabatic jacket. A thermoelectric device measures the temperature difference between the two cells and a feedback power is applied to maintain equal temperatures. In an ITC experiment, a ligand is titrated into a macromolecule in the sample cell at constant temperature. Upon each injection a heat change $(\Delta \mathrm{H})$ occurs proportionally to the amount of binding between the two molecules. The heat change is monitored by the feedback power ( $\mu \mathrm{cal})$ applied to maintain a constant temperature between the two cells. The feedback power applied per injection is monitored as a peak and the binding curve obtained by an ITC experiment is an integrated area of the peaks as a function of time. The binding curve is analyzed to determine the thermodynamic parameters of the interaction $\left(\mathrm{N}, \Delta \mathrm{H}\right.$ and $\left.\mathrm{K}_{\mathrm{d}}\right)$.

For ITC experiments, all proteins were dialysed against the same PBS buffer containing 20 $\mathrm{mM} \mathrm{NaH}{ }_{2} \mathrm{PO}_{4} / \mathrm{Na}_{2} \mathrm{HPO}_{4}, 200 \mathrm{mM} \mathrm{NaCl}$ and $1 \mathrm{mM}$ DTT. Protein concentrations were determined by absorption at $280 \mathrm{~nm}$. Protein samples were pre-incubated at $20^{\circ} \mathrm{C}$ and degassed prior to experiments. Experiments were performed at $25^{\circ} \mathrm{C}$ with 20 injections of $15 \mu \mathrm{L}$ volume each. The baseline corrected raw data was analyzed with MicroCal Origin 7.0 and the binding curves were fitted by using a non-linear least squares fit for a singlesite binding model. The competitive ITC experiments were analyzed as described previously (Velazquez-Campoy and Freire, 2006).

\subsubsection{CD spectroscopy}

Since proteins are chiral molecules, they are optically active. The optical activity can be measured by the difference in absorption for two types of circularly polarized light, i.e., left- and right-circularly polarized light. This is referred to as circular dichroism (CD) and is 


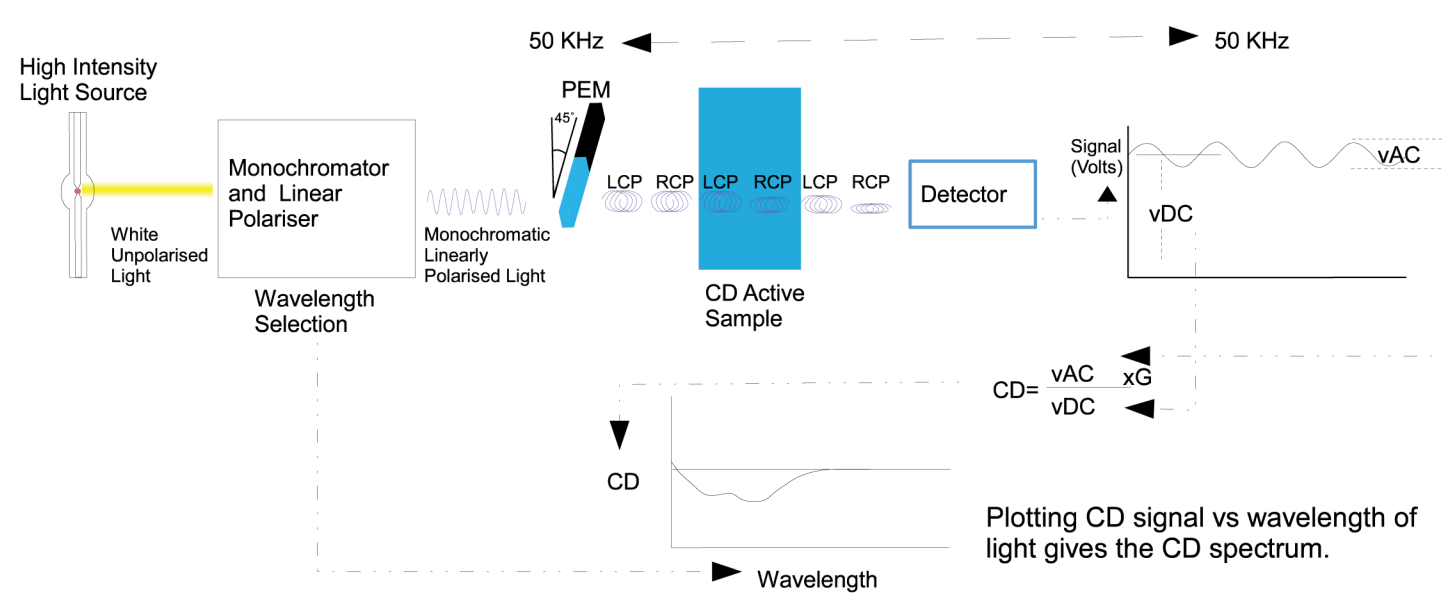

\section{Figure 2.1: Operation of a CD spectrometer}

Linearly polarized light of a specific wavelength is generated by the monochromator. BY PEM (photoelastic modulator), the beam is converted into left- (LCP) and right-circularly polarized light (RCP) alternating at 50 $\mathrm{KHz}$. Since chiral molecules absorb LCP or RCP preferentially, an unequal amount of LCP and RCP is collected at the detector. This signal oscillates at $50 \mathrm{KHz}$ as well. A lockin amplifier tuned at $50 \mathrm{KHz}$ produces vAC signal, whereas the average light intensity in the light detector is called vDC signal. CD (typically in millidegrees) is calculated by dividing the vAC signal with the vDC signal and multiplying the result with a calibration factor $(\mathrm{G})$. CD spectrum is a plot of the CD signal vs wavelength of light (Applied Photophysics).

detected by highly specialized absorption CD spectrometers (see Figure 2.1). The major chromophore of proteins is the amide bond joining two amino acids. Due to their regular backbone conformations, secondary structural elements of proteins such as a-helices and $\beta$-sheets, have distinctive CD spectra. $\alpha$-helices are characterized by a double minimum at 208 and 222 nm (Van Holde et al., 2006 p.471-483).

Proteins used in the CD experiments were in a buffer containing $20 \mathrm{mM}$ $\mathrm{NaH}_{2} \mathrm{PO}_{4} / \mathrm{Na}_{2} \mathrm{HPO}_{4}, 200 \mathrm{mM} \mathrm{NaCl}$ at 5-10 $\mu \mathrm{M}$ concentrations. To obtain CD spectra, the absorption signal was collected in a wavelength range of 190-250 $\mathrm{nm}$ at a bandwidth of 1 $\mathrm{nm}$. For kinetic measurements, the absorption signal was collected at $222 \mathrm{~nm}$ by obtaining a single data point per $5 \mathrm{sec}$. To perform thermal melts, the temperature was raised from $25^{\circ} \mathrm{C}$ to $95^{\circ} \mathrm{C}$ at $0.5^{\circ} \mathrm{C} / \mathrm{min}$ and the signal was measured at $222 \mathrm{~nm}$ with a step size of $0.5^{\circ} \mathrm{C}$.

CD signal was obtained in degrees of ellipticity $(\theta)$ and converted into mean residue ellipticity $\left([\theta]_{\mathrm{MR}}\right)$ by using the following formula: 
$[\theta]_{M R}=100 \times \theta /\left(C_{M R} \times 1\right)$

$\mathrm{C}_{\mathrm{MR}}=\mathrm{C} \times \mathbf{N}$

where $\mathbf{C}_{\mathrm{MR}}$ stands for mean residue concentration, $\mathbf{C}$ is the concentration of the protein molar, $\mathbf{N}$ is the number of amino acids in the protein and $\mathbf{l}$ is the pathlength in $\mathrm{cm}$.

\subsubsection{EPR spectroscopy}

EPR (Electron paramagnetic resonance) spectroscopy monitors the behavior of a nitroxide radical (spin label) attached at a specific position of a macromolecule and thus, is used as a probe for structural and dynamic information near the labeling site (reviewed in Hubbell et al, 2000). The most commonly used nitroxide molecule in EPR studies is methanethiosulfonate (1-oxy-2,2,5,5-tetramethylpyrrolinyl-3-methyl), also termed MTSL (Berliner et al., 1982) (Figure 2.2). In a continuous-wave (CW) EPR experiment, an external magnetic field $\left(B_{0}\right)$ is sweeped over the sample at a fixed microwave frequency. The resulting spectrum is portrayed as the first derivative of the absorption spectrum. Three types of motions contribute to the spectrum:

- Brownian rotational diffusion of the molecule

- Torsional rotation of the bonds that connect the spin-label to the molecule

- Backbone fluctuations at or near the labeling site

Nitroxide mobility is analysed via two parameters of the spectrum: the width of the central line and the splitting between the outer peaks. Both parameters increase as the mobility of nitroxide decreases (Hubbell et al., 2000).

Before starting the labeling reaction, DTT was removed from the protein solution by size exclusion chromatography using PD-10 columns (Amersham Pharmacia Biotech). Immediately after, the proteins were mixed with MTSL (labeling buffer: $20 \mathrm{mM}$ Tris $\mathrm{pH}$ $7.4,200 \mathrm{mM} \mathrm{NaCl}$ and $2 \mathrm{M}$ urea) and incubated for about 2 hours at room temperature. Unreacted spin-label was removed using PD-10 columns in the labeling buffer. Since rapid renaturation of the proteins caused oligomerization, in order to remove urea, the labeled proteins were dialysed overnight against a buffer containing $20 \mathrm{mM}$ Tris $\mathrm{pH}$ 7.4, 


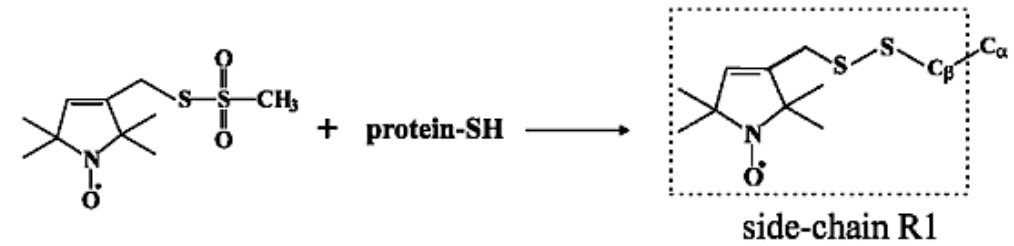

Figure 2.2: The spin-labeling reaction of MTSL

$200 \mathrm{mM} \mathrm{NaCl}$. The labeled proteins were concentrated in the Microcons after dialysis. To form complexes with Sly1p, labeled proteins were mixed with Sly1p in a molar ratio of 1:2 in the presence of $10 \%(\mathrm{v} / \mathrm{v})$ glycerol. Spectra were collected using a X/Q-band (9 $\mathrm{GHz} / 34 \mathrm{GHz}$ ) EMX CW EPR spectrometer by Dr. Giuseppe Sicoli (Max Planck Institute for Biophysical Chemistry, Göttingen).

\subsubsection{Fluorescence spectroscopy}

Protein labeling with fluorescent dyes

DTT was removed from the proteins by dialysis overnight, in a buffer containing $20 \mathrm{mM}$ $\mathrm{NaH}_{2} \mathrm{PO}_{4} / \mathrm{Na}_{2} \mathrm{HPO}_{4}, 200 \mathrm{mM} \mathrm{NaCl}$ and $100 \mu \mathrm{M}$ TCEP. Texas Red Maleimide or Oregon Green 488 iodoacetamide was dissolved in an organic solvent (DMF, 2,4-dimethyl formamide) before addition to proteins at $\sim 10$-fold molar excess. The labeling reaction was carried out at room temperature for $\sim 2$ hours and stopped with $10 \mathrm{mM}$ DTT. Unreacted dye was removed by size-exclusion chromatography using PD-10 columns. The labeled proteins were visualized on 15\% SDS-PAGE or 10\% Schagger gels (Schagger and von Jagow, 1987). To determine the labeling efficiency, the concentration of the fluorophore was measured by absorption spectroscopy and the concentration of the protein was measured by Bradford assay (Bradford, 1976).

\section{Fluorescence anisotropy}

When fluorescently labeled molecules are excited by linearly polarized light, only dye molecules with transition dipoles aligned parallel to the plane of polarization, will selectively absorb the light. The polarization of the emitted light will depend on the 
following factors:

- The orientation of the emitting transition dipole with respect to the absorbing transition dipole

- The amount of molecular rotation that happens during the fluorescence lifetime

As a result, the fluorescence light is partially depolarized (Van Holde et al., 2006 p.517524). Experimentally, the degree of polarization is determined by measuring fluorescence intensities parallel and perpendicular relative to the plane of linearly polarized excitation light.

In this study, all anisotropy experiments were carried out in a Fluorolog 3 spectrometer (Model FL322, Horiba Jobin Yvon) in T-configuration. In the T-format, horizontally and vertically polarized components of the emission are simultaneously acquired on two separate detecting systems. Polarization is expressed in terms of fluorescence anisotropy $(R)$ with the following equation:

$$
R=\left(I_{\mathrm{vv}}-G \times I_{\mathrm{vh}}\right) /\left(I_{\mathrm{vv}}+2 \times G \times I_{\mathrm{vh}}\right)
$$

where $I_{\mathrm{vV}}$ is the intensity of the vertically polarized fluorescence emission (second index $v$ ) and $I_{\mathrm{vh}}$ is that of the horizontally polarized fluorescence emission (second index $h$ ) upon excitation with a vertically polarized light (first index $v$ ). $G$ is an instrumental for the relative sensitivities of the two detecting systems, i.e., the detecting systems for the vertically and horizontally polarized emission. It is described in the following formula:

$$
G=I_{\mathrm{hv}} / I_{\mathrm{hh}}
$$

where $I_{\mathrm{hv}}$ is the intensity of the vertically polarized emission and $I_{\mathrm{hh}}$ is that of the horizontally polarized emission upon excitation with a horizontally polarized beam (Lakowicz, 2000 p.291-319).

Oregon Green 488 iodoacetamide was used for all anisotropy experiments described in this study. Samples were excited at $488 \mathrm{~nm}$ and the emission collected at $520 \mathrm{~nm}$. Either ultra-micro fluorescence cells (V: $100 \mu \mathrm{l})$ or semi-micro cuvettes (V: $1400 \mu \mathrm{l})$ were used (Hellma). Slit width was in the range of 3-6 $\mathrm{nm}$. All proteins were dialysed against the experimental buffer $\left(20 \mathrm{mM} \quad \mathrm{NaH}_{2} \mathrm{PO}_{4} / \mathrm{Na}_{2} \mathrm{HPO}_{4}\right.$ and $\left.200 \mathrm{mM} \mathrm{NaCl}\right)$ prior to measurements. 
Förster resonance energy transfer (FRET)

FRET takes place under favorable circumstances which allow the transfer of the excitation from one fluorophore (donor) to another (acceptor). Primary conditions for FRET are as follows:

- Donor and acceptor fluorophores must be in close proximity (10-100 ̊).

- Donor and acceptor transition dipole orientations must be approximately parallel.

- There should be an appreciable overlap between the fluorescence spectrum of the donor and the absorption spectrum of the acceptor.

FRET efficiency is described in the following formula:

$$
\text { FRET efficiency }=1 /\left[1+\left(r / R_{0}\right)^{6}\right]
$$

where $r$ is the distance between the dye molecules and $R_{0}$ is the distance for $50 \%$ efficiency of transfer. $R_{0}$ is characteristic for the donor-acceptor pair, the relative orientation of their transition dipoles and the medium (Van Holde et al., 2006 p.516-517).

FRET experiments in this study were performed using a Fluorolog 3 spectrometer (Model FL322, Horiba Jobin Yvon) in T-configuration. The T-format allowed simultaneous acquisition of the donor and the acceptor fluorescence on two detecting systems. Fluctuations caused by the light source were corrected by monitoring lamp output using the reference detector, $R$, and using signal ratio $S / R$. Oregon Green 488 iodoacetamide and Texas Red maleimide was the FRET pair chosen. The sample was excited at $488 \mathrm{~nm}$ and emission was collected at $520 \mathrm{~nm}$ and $610 \mathrm{~nm}$ on two separate detectors. Semi-micro cuvettes (V: $1400 \mu \mathrm{l})$ were used. Sample was in a buffer containing $20 \mathrm{mM}$ $\mathrm{NaH}_{2} \mathrm{PO}_{4} / \mathrm{Na}_{2} \mathrm{HPO}_{4}$ and $200 \mathrm{mM} \mathrm{NaCl}$.

\subsubsection{Other methods}

\section{Gel electrophoresis}

SDS-PAGE (12\% and 15\%) and native (9\%) gels were prepared by mixing the components shown in Table 2.2. SDS was omitted from the native gel buffers and the running buffer. After electrophoresis, the gel was stained in a standard solution $(50 \%(\mathrm{v} / \mathrm{v})$ methanol, 
Table 2.2: Composition of SDS-PAGE and native gels

\begin{tabular}{r|c|c|c|c} 
& \multicolumn{3}{|c|}{ Separating gel (4 gels) } & Stacking gel (4 gels) \\
\cline { 2 - 5 } & $\mathbf{9 \%}$ & $\mathbf{1 2} \%$ & $\mathbf{1 5 \%}$ & $3,75 \%$ \\
\hline $\mathbf{d H}_{\mathbf{2}} \mathbf{O}$ & $4.4 \mathrm{ml}$ & $3.4 \mathrm{ml}$ & $2.4 \mathrm{ml}$ & $12.28 \mathrm{ml}$ \\
\hline Gel buffer & $2.5 \mathrm{ml}$ & $2.5 \mathrm{ml}$ & $2.5 \mathrm{ml}$ & $5 \mathrm{ml}$ \\
\hline Acrylamide/ Bisacrylamide & $3 \mathrm{ml}$ & $4 \mathrm{ml}$ & $5 \mathrm{ml}$ & $2.5 \mathrm{ml}$ \\
\hline $\mathbf{1 0} \% \mathbf{A P S}$ & $5 \mu \mathrm{l}$ & $5 \mu \mathrm{l}$ & $5 \mu \mathrm{l}$ & $20 \mu \mathrm{l}$ \\
\hline TEMED & $100 \mu \mathrm{l}$ & $100 \mu \mathrm{l}$ & $100 \mu \mathrm{l}$ & $200 \mu \mathrm{l}$
\end{tabular}

*Stacking gel buffer: $0.5 \mathrm{M}$ Tris pH: 6.8 (+0.4\% SDS)

*Separating gel buffer: $1.5 \mathrm{M}$ Tris $\mathrm{pH}: 8.8$ (+0.4\% SDS)

10\% (v/v) acidic acid and 0.2\% (w/v) Coomassie Brilliant Blue R-250). The gel was destained in Destain I (50\% (v/v) ethanol and 10\% (v/v) acidic acid) for about $15 \mathrm{~min}$, and then in Destain II (5\% (v/v) methanol and 12. 5\% (v/v) acetic acid) until the background staining is invisible.

\section{Determination of protein concentrations}

Protein concentrations were in general determined by absorption spectroscopy using either UV-Photometer UV-2401 PC (Kyoto, Japan) or Nanodrop (Thermo Scientific). The absorption spectra were obtained between a wavelength range of 220-350 $\mathrm{nm}$. The protein concentration was determined using the absorption at $280 \mathrm{~nm}$ and the extinction coefficients indicated in the SwissProt database (Wilkins et al., 1999 p.531-552). The concentration of fluorescently labeled proteins were determined by Bradford assay (Bradford, 1976). 


\section{Chapter 3 Results}

\subsection{Biochemical characterization of Sly1p interaction with the ER- Golgi SNAREs}

Yeast Sly1p is an essential regulator of vesicular trafficking events between ER and Golgi (Ossig et al., 1991). As for many other SM proteins, the primary target for Sly1p has been shown to be a syntaxin (i.e., a Qa-SNARE), Sed5p (Grabowski and Gallwitz, 1997). Sly1p has been suggested to regulate the assembly of ER-Golgi SNAREs by a tight interaction with Sed5p and, possibly by much weaker associations with the nonsyntaxin SNAREs (Peng and Gallwitz, 2004). On the other hand, little is known about the molecular mechanism of Sly1p regulation. In order to understand the cellular function of Sly1p in more detail, I initially investigated the binding mode between Sly1p and Sed5p. This groundwork assisted to explain the interplay with the nonsyntaxin-SNAREs. Finally, I validated the interaction between Sly1p and another syntaxin, Ufe1p, which likely serves during the retrograde transport from Golgi to ER.

\subsubsection{Elucidating the conformation of individual Sed5p}

It has been shown for several syntaxins that they can adopt a so-called closed conformation, in which the Habc domain of the syntaxin folds back against the SNARE motif. Structural and biochemical evidence from neuronal Syntaxin 1a and yeast Sso1p demonstrate that the closed conformation of the syntaxins impede the accessibility of their respective SNARE motifs (Nicholson et al., 1998, Munson et al., 2000, Misura et al., 2000, 
Burkhardt et al., 2008, Furgason et al., 2009). Whereas Sso1p adopts a closed conformation as individual protein, Syntaxin 1a adopts a closed conformation when it is bound to Munc18a (Misura et al., 2000, Burkhardt et al., 2008). It is debated, however, whether uncomplexed Syntaxin 1a is mostly closed or open (Margittai et al., 2003b, Chen et al., 2008). In addition, it is unclear whether Munc18a bound Syntaxin 1a can switch into a configuration that is accessible for its partner SNARE proteins.

Like many other syntaxins, the SNARE motif (also referred to as H3 domain) of Sed5p is preceded by an N-terminal regulatory domain, which consists of a short N-peptide motif (residues 1-21), the Habc domain (53-168), and a linker region (168-258) (Yamaguchi et al., 2002) (Figure 3.1). Previous biochemical data suggest that yeast Sed5p might adopt a closed conformation (Mossessova et al., 2003), but no direct evidence for this configuration has been brought by so far. If already individual Sed5p can adopt a closed conformation, this configuration might also play a role during Sly1p binding, maybe similar to the configuration found in the crystal structures of the Munc18a/ Syntaxin 1a complexes (Misura et al., 2000, Burkhardt et al., 2008, Burkhardt et al., 2011). This would be an important information as it would probably affect the ability of Sed5p to assemble with its partner SNAREs. Therefore, prior to investigating the binding mechanism between Sly1p and Sed5p, I wanted to find out whether individual Sed5p resides in a closed conformation.

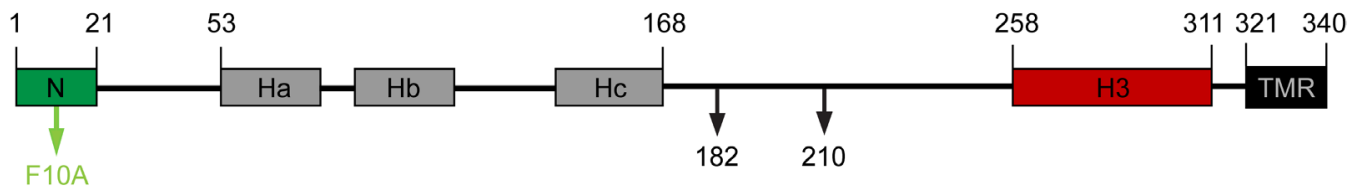

Figure 3.1: Schematic diagram of the domain structure of Sed5p

The domain structure shown above is depicted partly according to previous NMR studies (Yamaguchi et al., 2002). The short N-peptide motif (residues 1-21) is shown in green and the F10A point mutation that drastically affects Sly1p binding is highlighted. The individual Habc helices (53-168) detected by NMR is shown in grey, yet the C-terminal boundary of Hc helix could not be precisely defined in this study (Yamaguchi et al., 2002). Indeed, a sequence alignment of Sed5p from several fungi (Kienle et al., 2009) reveals conservation up to residue 182 (see Appendix A.1). In the present study, a fragment was used as the Nterminal domain of Sed5p, which also contained a stretch of the linker region between Habc and SNARE motif (residues 1-210). The SNARE motif (258-311) is shown in red. Its boundaries are not precisely defined, but according to SNARE database (http://bioinformatics.mpibpc.mpg.de/snare) (Kloepper et al., 2007), the core helix starts from residue 258. The SNARE (H3) motif is connected to the transmembrane domain (residues 321-340, shown in black) via a short linker. 
As a first step, both the entire cytosolic portion of Sed5p (residues 1-320) and a fragment comprising only its N-terminal domain (residues 1-210) were bacterially produced and purified. In order to monitor the structures of the protein fragments and to measure their conformational stabilities, circular dichroism (CD) spectroscopy was employed. CD spectroscopy is a powerful tool to observe the structural elements of a protein and to monitor their changes. Indeed, various secondary structural elements such as a- helices, $\beta$-sheets or random coils have specific CD signatures in the far-UV range $(170-250 \mathrm{~nm})$. For example, pronounced double minima at $\sim 208 \mathrm{~nm}$ and $\sim 222 \mathrm{~nm}$ signify the presence of a-helices. CD spectra from Sed5p (1-320) and of Sed5p (1-210) revealed that both proteins had significant a-helical content (Figure 3.2 inset). This is not very surprising considering the autonomously folded three-helical bundle structure of the Habc domain.

Afterwards, conformational stabilities of Sed5p fragments were tested by thermal denaturation using CD spectroscopy (Figure 3.2). The entire cytosolic portion of Sed5p unfolded within a single transition at $\operatorname{Tm} \approx 72{ }^{\circ} \mathrm{C}$, whereas the N-terminal portion of Sed5p (residues 1-210) melted at a much lower temperature $\left(\operatorname{Tm} \approx 60^{\circ} \mathrm{C}\right)$. This suggests that the $\mathrm{N}$-terminal portion of Sed5p is stabilized in the presence of its SNARE motif. A similar behavior has been observed for yeast exocytic Sso1p, and proposed to indicate a tight

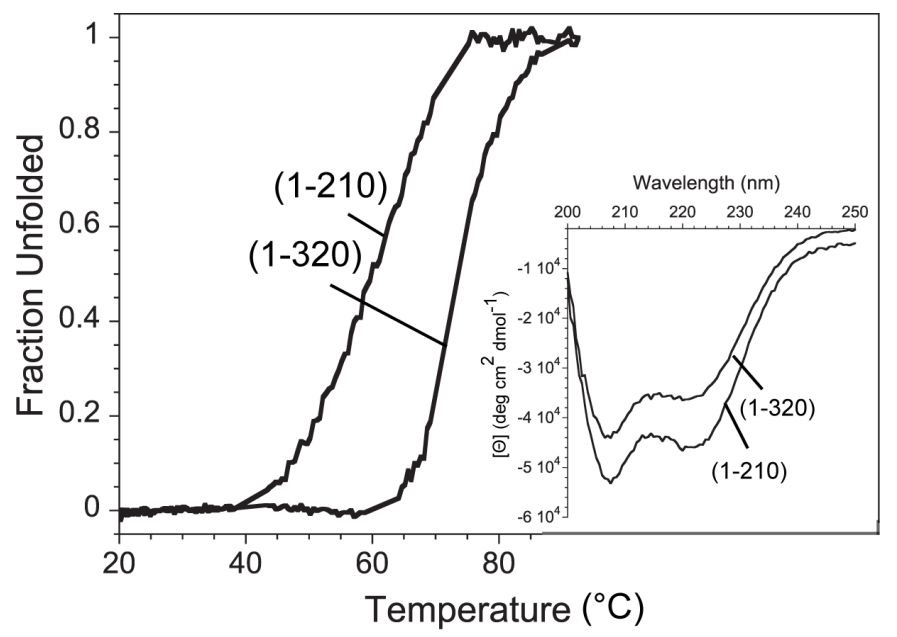

Figure 3.2: Secondary structure characterization of Sed5p fragments using CD spectroscopy

Thermal denaturation of Sed5p fragments, spanning the entire cytosolic portion (residues 1-320) and the Nterminal domain (residues 1-210) of the syntaxin. Inset shows the far-UV CD spectra, obtained from the same fragments. For all measurements, the proteins were diluted up to $\sim 5 \mu \mathrm{M}$ in a buffer containing $20 \mathrm{mM}$ $\mathrm{NaH}_{2} \mathrm{PO}_{4} / \mathrm{Na}_{2} \mathrm{HPO}_{4} \mathrm{pH}: 7.4$ and $200 \mathrm{mM} \mathrm{NaCl}$. Experiments were performed in $0.1 \mathrm{~cm}$-cuvettes and for thermal denaturation the temperature was raised from $20{ }^{\circ} \mathrm{C}$ up to $95{ }^{\circ} \mathrm{C}$. Unfolding was monitored by the increase in CD ellipticities at $222 \mathrm{~nm}$. The denaturation curves were analysed as described previously (Pace et al., 1989). 
A

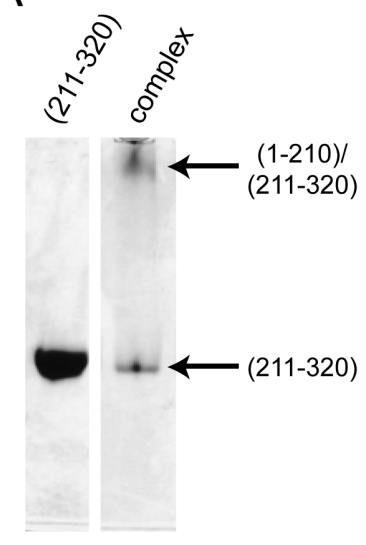

B

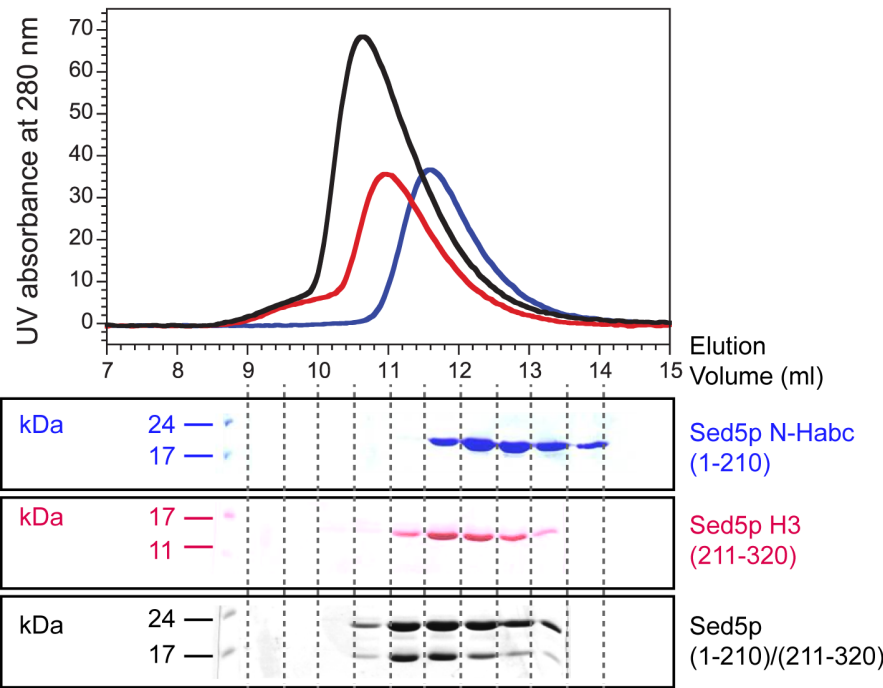

Figure 3.3: Biochemical analysis of the interaction between the Sed5p SNARE motif and the Sed5p Nterminal domain

A. Interaction between Sed5p (1-210) and Sed5p (211-320) was observed by native gel electrophoresis. 100 nmols of each fragment were applied on a $9 \%$ native gel separately or as a mixture. Sed5p (1-210) could not enter the gel, possibly since it was basic. Sed5p (211-320) and a new band, corresponding to a complex between Sed5p (1-210) and (211-320) were visualized on the native gel. B. $\sim 70 \mu \mathrm{M}$ of Sed5p (1-210) and Sed5p (211-320) were applied to a Superdex 75 HR 10/ 30 column either separately or as a mixture. All gel filtration experiments were performed with a flow rate of $0.7 \mathrm{ml} / \mathrm{min}$ and an injection volume of $450 \mu \mathrm{l}$. The buffer was containing $20 \mathrm{mM} \mathrm{NaH} \mathrm{PO}_{4} / \mathrm{Na}_{2} \mathrm{HPO}_{4} \mathrm{pH}$ : 7.4 and $200 \mathrm{mM} \mathrm{NaCl}$. In each case, $0.5 \mathrm{ml}$ peak fractions were collected and analysed by SDS-PAGE. The elution profiles and the Coomassie stainings of each experiment are colour-coded as follows: Sed5p (1-210) in blue, Sed5p (211-320) in red and a mixture of both in black. Note that a portion of Sed5p (211-320) is eluted as oligomers.

interaction of the Habc and the SNARE domain (Fiebig et al., 1999). Indeed, the crystal structure of Sso1p then revealed that the protein adopts a tight closed conformation (Munson et al., 2000). Accordingly, the thermal stabilities of the Sed5p fragments support the notion that Sed5p exists in a closed state in solution.

In order to provide further evidence for the intramolecular interaction within Sed5p, two fragments of Sed5p were used: Sed5p (1-210) containing the N-terminal portion and Sed5p (211-320) containing the SNARE motif. Initially, native gel electrophoresis was used to test the interaction between the two Sed5p fragments (Figure $3.3 \mathrm{~A}$ ). Indeed, a protein complex containing the two fragments of Sed5p appeared as a new band on the native gel. Then, the interaction of two Sed5p fragments was examined by size-exclusion chromatography (Figure 3.3 B). For this, equal amounts of Sed5p (1-210) and Sed5p (211320) were run on a Superdex 75 gel filtration column separately or as a mixture. The gel filtration elution profiles of the proteins reveal that the two fragments form a complex. 


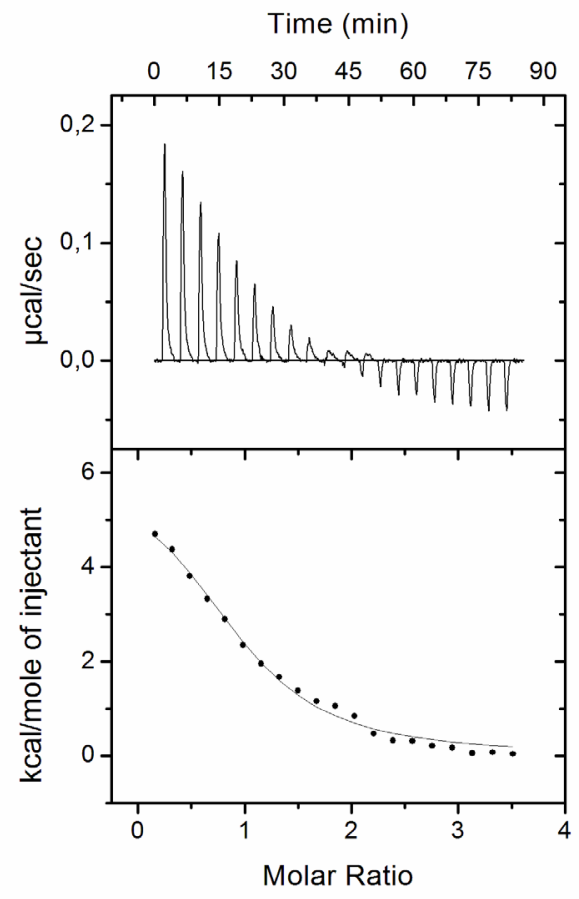

\section{Figure 3.4: Calorimetric titration of Sed5p SNARE motif with the Sed5p N-terminal domain}

Diagram shows an ITC experiment, performed with $10 \mu \mathrm{M}$ Sed5p (211-320) in the cell and $150 \mu \mathrm{M}$ Sed5p (1210) in the syringe. Sed5p (1-210) includes the N-terminal domain of the syntaxin, whereas Sed5p (211-320) spans the SNARE motif (see Figure 3.1). The upper panel of the diagram shows the base-line corrected raw data corresponding to heat change over time ( $\mu \mathrm{cal} / \mathrm{s})$. The lower panel is obtained by integration of area under the peaks and a normalization according to the amount of ligand injected (kcal/ mole). Each injection event results in heat uptake until Sed5p (211-320) amount in the cell starts to be limiting. When all available Sed5p (211-320) is in complex, each injection event results in heat release caused by the dilution of Sed5p (1$210)$ injected. Such endothermic protein-protein interactions are entropy-driven and they are thought to be driven by desorption of water from the interacting molecules. In other words, the non-polar regions of Sed5p fragments could interact to minimize the binding of solvent to the non-polar residues. See table 3.1 for energetical parameters of the reaction.

Finally, the interaction between the two Sed5p fragments was tested by isothermal titration calorimetry (ITC). An ITC experiment measures the heat change upon addition of a ligand to a macromolecule at constant temperature. The titration curve obtained provides important thermochemical parameters of a binding reaction such as the enthalpy change $\left(\Delta \mathrm{H}^{0}\right)$, the dissociation constant $\left(\mathrm{K}_{\mathrm{d}}\right)$ and the stoichiometry $(\mathrm{N})$. The two Sed5 fragments interacted with an unfavourable enthalpy (+ $6 \mathrm{kcal} /$ mole) at a 1:1 stoichiometry (Figure 3.4). The affinity of the two Sed5p fragments was rather low $\left(K_{d}\right.$ of $\left.\approx 2.7 \mu \mathrm{M}\right)$ (Table 3.1). Hence, the linker region inbetween the two Sed5p segments could play an important role for stabilizing the closed conformation. 


\subsubsection{Analysis of the interaction between Sly1p and Sed5p}

Up to now, Sly1p is thought to interact only with the short N-terminal peptide of Sed5p (Bracher and Weissenhorn, 2002, Yamaguchi et al., 2002), although the earlier studies could not rule out that Sly1 might also interact weakly with the Habc domain (Yamaguchi et al., 2002) and/ or the SNARE motif (Bracher and Weissenhorn, 2002). Interestingly, it was observed that Sly1p-bound Sed5p does not form nonphysiological SNARE complexes in vitro (Peng and Gallwitz, 2002). Additionally, the high-affinity binding of N-peptide to Sly1p seems to be dispensable in vivo (Peng and Gallwitz, 2004). These findings support the notion that the Sly1p/ Sed5p interaction could involve not only the N-peptide region of Sed5p, but also other parts of the syntaxin as well. Indeed, it has been reported that several SM proteins such as Munc18a, Munc18b, Munc18c, Monosiga Munc18, Unc18, Vps45 or Vps45p make use of two binding sites when interacting with their cognate syntaxins: the N-peptide and the closed conformation (Burkhardt et al., 2008, Furgason et al., 2009, Aran et al., 2009, Johnson et al., 2009, Burkhardt et al., 2011, Peng et al., 2010). Since my results presented in the previous section suggest that Sed5p folds into a closed conformation in solution, I wanted to investigate whether Sly1p can interact with the closed form of Sed5p.

In order to re-investigate the binding mode between the two proteins, Sly1p and additional truncated and mutated forms of Sed5p were produced. Note that all constructs of Sed5p lacked the transmembrane domain. Using ITC, I found that the entire cytosolic region of Sed5p (residues 1-320), binds to Sly1p with an enthalpy of $\Delta H \approx-24 \mathrm{kcal} / \mathrm{mole}$ and a high affinity $\left(\mathrm{K}_{\mathrm{d}} \approx 0.25 \mathrm{nM}\right)$ (Table 3.1, Figure 3.5 A). To determine which regions of Sed5p contribute to binding, $\mathrm{N}$ - and C-terminally truncated variants of the protein were used in ITC experiments. No binding was observed between Sly1p and Sed5p lacking the N-peptide (residues 21-324) (Table 3.1, Figure 3.5 A). Therefore, N-peptide binding indeed appears to be essential for Sly1p interaction. Supporting this notion, I observed that Sly1p does also not bind to a Sed5p fragment containing only the SNARE motif (residues 211320) (Table 3.1).

Interestingly, the C-terminally deleted constructs of Sed5p bound to Sly1 $p$ with an affinity comparable to that of the entire cytosolic region. The isolated N-peptide, Sed5p (1-21), bound with a $\mathrm{K}_{\mathrm{d}} \approx 1.47 \mathrm{nM}$ and Sed5p (1-210), which covers also the Habc domain and a part of the linker, bound with a $\mathrm{K}_{\mathrm{d}} \approx 0.24 \mathrm{nM}$ (Table 3.1, Figure 3.5 A). It is striking, 

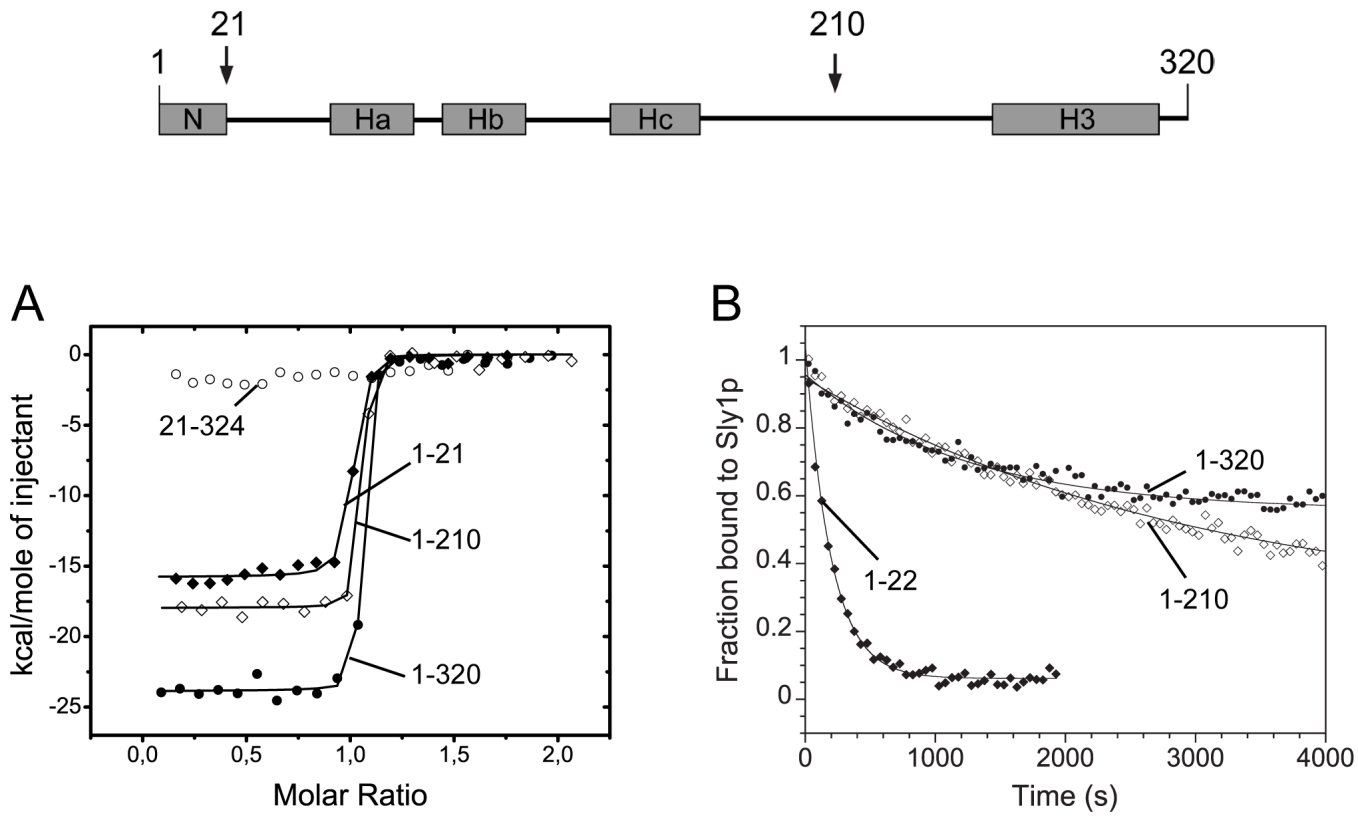

Figure 3.5: Biochemical analysis of Sly1p interaction with truncated Sed5p variants

A. Titration curves obtained by ITC experiments, performed between Sly1p and various truncated forms of Sed5p (21-324, 1-21, 1-210, 1-320). In all experiments, $15 \mu \mathrm{M}$ Sed5p variant in the syringe (30 $\mu \mathrm{M}$ Sed5p 21-324 as only exception) was injected into the cell containing $2 \mu \mathrm{M}$ Sly1p. For clarity, only the lower panels of superimposed ITC diagrams are shown. See table 3.1 for thermodynamic parameters of the reactions. B. Comparison of the off-rates of different Sed5p variants from Sly1p measured by competitive dissociation. Firstly, $\sim 50 \mathrm{nM}$ fluorophore (Oregon Green or Texas Red)-attached Sed5p variants were mixed with saturating amounts of Sly1p. Then, an excess of unlabeled Sed5p variants $(\sim 2 \mu \mathrm{M})$ were added and the decrease in fluorescence anisotropy was measured. Fractions of labeled Sed5p variants bound to Sly1p, were calculated according to anisotropy values of the fully-bound and the non-bound states of the Sed5p variants. The off-rates of different Sed5p segments were calculated from single exponential fits. Above the figures, the truncation points of Sed5p mutants are marked on the schematic drawing.

however, that the heat release upon Sly1p binding was smaller when C-terminally truncated Sed5p constructs were used. For instance, the binding enthalpy of Sed5p (1-210) was $\approx-18 \mathrm{kcal} /$ mole and that of Sed5p (1-21) was $\approx-16 \mathrm{kcal} /$ mole, whereas that of the Sed5 (1-320) was - $24 \mathrm{kcal} /$ mole (Table 3.1). This difference in binding enthalpy can very likely be attributed to a loss of contributing interaction surface. This strongly suggests that not only the N-peptide but also the remaining portion of Sed5p contributes to Sly1p interaction. Note that comparable differences had been observed when the interaction between Syntaxin 1a and Munc18a was analyzed using ITC (Burkhardt et al., 2008). In fact, those findings were instrumental to discover that the Munc18/ Syntaxin 1a complex contains two spatially separated binding sites.

As explained above, the affinities determined for the different Sed5 fragments using ITC were in the nanomolar range. It needs to be mentioned, however, that the dissociation 
constants could not be accurately determined, since they were at the lower limit of the ITC instrument. Therefore, to gain an independent read-out for the affinity between the two proteins, I used another approach, fluorescence anisotropy. Fluorescence anisotropy is a powerful tool to monitor protein-protein interactions. In an anisotropy experiment, a fluorophore is attached to a specific position of one of the interacting protein molecules. After excitation by plane-polarized light, rotation and tumbling of the fluorophore causes emission in a different plane from the excitation light. When the labeled-protein molecule is bound to another protein molecule, the rotational freedom of the fluorophore can change. This can occur when the fluorophore binds to a bigger molecule, which moves slower. Alternatively, this can occur when the fluorophore resides in a more rigid microenvironment upon complex formation. When the fluorophore is more stationary, the emitted light is also less depolarized. As a result, protein-protein interactions can lead to an increase in fluorescence anisotropy.

In order to use in fluorescence measurements, the truncated Sed5p variants (residues 1-22, 1-210 or 1-320) were labeled with fluorophores at position 22, i.e. right after the $\mathrm{N}$-peptide motif. When the labeled fragments were mixed with Sly1p, a clear anisotropy increase was observed for all three variants, denoting binding. The on-rates of all different Sed5p variants were rapid and comparable (data not shown). To observe the dissociation of the Sed5p fragments, an excess amount of the respective unlabeled Sed5p fragment was

Table 3.1: Thermodynamic parameters obtained by all ITC experiments in this study

\begin{tabular}{l|l|l|l|l} 
CELL & SYRINGE & $\left.\mathbf{K}_{\mathbf{d}} \mathbf{( n M}\right)$ & $\Delta \mathbf{H} \mathbf{( k c a l / ~ m o l e )}$ & $\mathbf{N}$ \\
\hline Sly1p & Sed5p (1-320) & $0.25 \pm 0.12$ & $-23.9 \pm 0.16$ & 1.02 \\
\hline Sly1p & Sed5p (1-210) & $0.24 \pm 0.35$ & $-18 \pm 0.16$ & 1.00 \\
\hline Sly1p & Sed5p (1-21) & $1.47 \pm 0.45$ & $-16 \pm 0.13$ & 0.98 \\
\hline Sly1p & Sed5p (21-324) & - & - & - \\
\hline Sly1p & Sed5p (211-320) & - & - & - \\
\hline Sly1p & Sed5p F10A (1-320) & - & - & 1.09 \\
\hline Sly1p & Sed5p F10A (1-21) & $0.48 \pm 0.49$ & $-22.7 \pm 0.20$ & - \\
\hline Sly1p & Sed5p (1-210)/ (211-320) complex & $0.23 \pm 0.30$ & $-20.3 \pm 0.22$ & 0.92 \\
\hline Sly1p & SNARE complex with Sed5p (1-320) & - & 0.88 \\
\hline Sly1p & SNARE complex with Sed5p (211-320) & - & - & - \\
\hline Sly1p & Ufe1p (1-21) & $34.4 \pm 5.2$ & $-15.8 \pm 0.23$ & 1.02 \\
\hline Sly1p/ Ufe1p (1-21) & Sed5p (1-320) & $0.17 \pm 0.09$ & $-21.9 \pm 0.37$ & 0.85 \\
\hline Sly1p/ Ufe1p (1-21) & Sed5p (1-210) & - & - & - \\
\hline Sed5p (211-320) & Sed5p (1-210) & $2700 \pm 400$ & $+6.13 \pm 0.34$ & 0.98
\end{tabular}


added. The dissociation was observed as a decrease in anisotropy and the off- rates of Sed5p constructs were determined by fitting the change using a single exponential fit (Figure $3.5 \mathrm{~B}$ ). The determined dissociation-rates were as follows: Sed5p $(1-22) \approx 0.0051 \mathrm{~s}^{-1}$, Sed5p $(1-210) \approx 0.00034 \mathrm{~s}^{-1}$ and Sed5p $(1-320) \approx 0.00064 \mathrm{~s}^{-1}$. It is evident from the off-rates that the longer Sed5p fragments Sed5p (1-210) and (1-320), were held much tighter by Sly1p compared to the sole N-peptide region, Sed5p (1-22). Accordingly, not only the Npeptide, but at least the Habc motif of Sed5p also interacts with Sly1p. It is not absolutely clear, however, whether Sed5p SNARE motif also contributes to binding, because the offrates of Sed5p (1-320) and Sed5p (1-210) are quite similar. Overall, my results confirm earlier studies which have suggested that the N-peptide motif of Sed5p is essential and is the major source of the high-affinity Sly1p interaction (Yamaguchi et al., 2002, Bracher and Weissenhorn, 2002). However, my data indicates that other regions of Sed5p bind to Sly1p as well.

Next, I wanted to particularly determine whether the SNARE motif of Sed5p could participate in binding. Since the binding can be weak or transient, I chose a sensitive biophysical tool, site-directed spin labeling. A site-directed spin labeling experiment involves attachment of a nitroxide reagent to a molecule at a specific position. The electron paramagnetic resonance (EPR) spectrum of the labeled molecule depends on the microenvironment of the spin-label. By analysis of an EPR spectrum, the spin-label site can be distinguished as loop, exposed, buried, or in tertiary contact. Generally speaking, sharp and narrowly spaced peaks are obtained from mobile regions, whereas strongly broadened peaks denote regions that are buried or in tertiary contact.

To observe rotational freedom and tertiary contact of the Sed5p SNARE motif, using EPR, I attached nitroxides to several positions on the SNARE domain of Sed5p (1-320). EPR spectra were collected from labeled Sed5p molecules, when they were isolated or were in complex with Sly1p (Figure 3.6). By comparing the EPR spectra obtained from a specific position, I wanted to determine the tertiary contact of Sly1p in this region. I found that the spectra obtained from all individual Sed5p molecules exhibited sharp and narrowly spaced lines. This suggests that the spin-labels were located on a loop or on a helixsurface. In fact, the crystal structure from Munc18a /Syntaxin1 was used as a guide to choose the spin-label positions (Misura et al., 2000, Burkhardt et al., 2008). If Sly1p/ Sed5p complex had a similar configuration, the nitroxides were expected to lie on the surface of the $\mathrm{H} 3$ helix and in close proximity to Sly1p. Interestingly, the EPR spectra of the Sly1p 

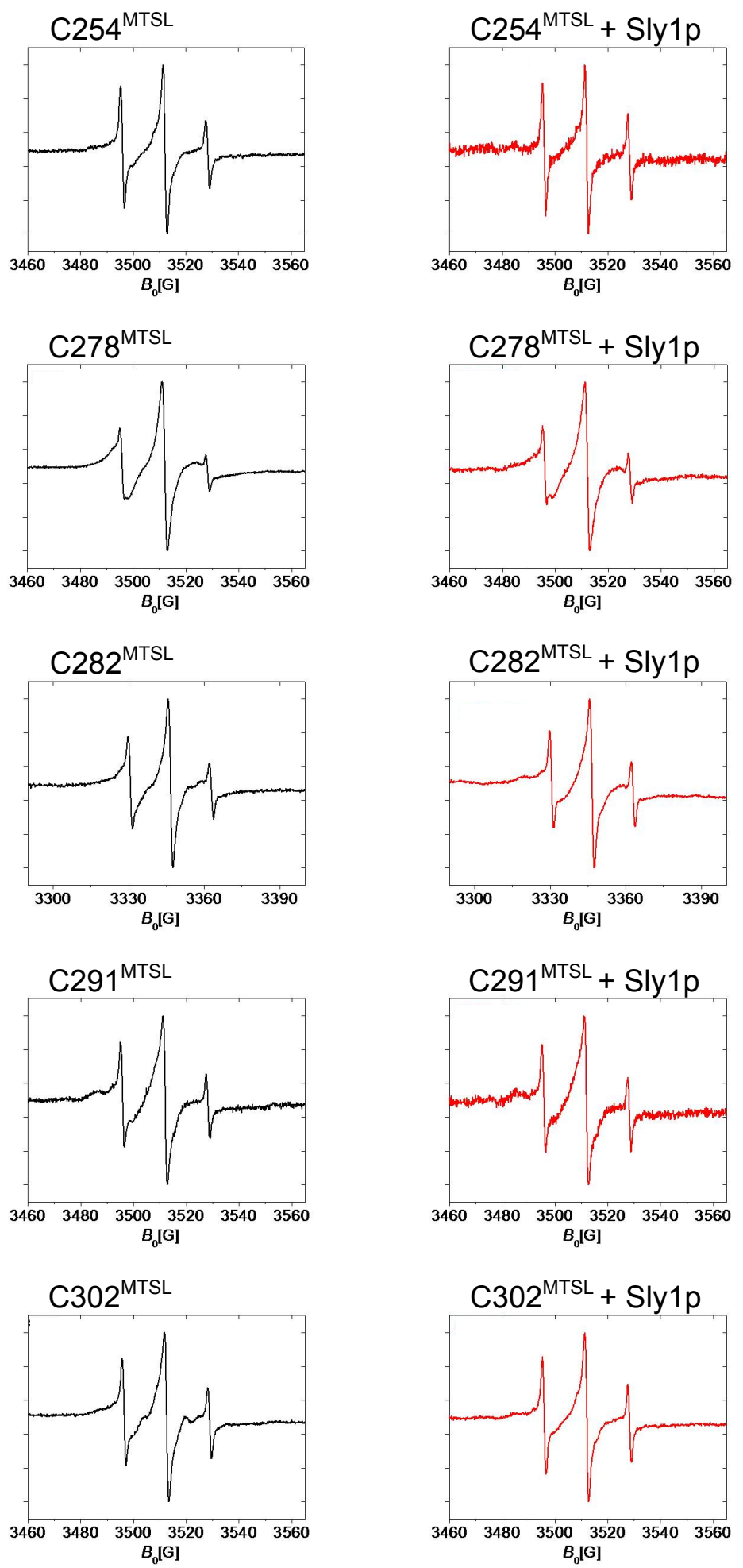

Figure 3.6: EPR spectra of individual Sed5p or in complex with Sly1p

Spin-labels were attached to the following positions on the SNARE motif of Sed5p; C254, C278, C282, C291 and C302. Next, continuous wave-EPR (CW-EPR) spectra were collected from 10-15 $\mu \mathrm{M}$ of spin-labeled Sed5p molecules (black lines). For comparison, EPR spectra of Sly1p bound Sed5p molecules were obtained as well (red lines). Complexes were formed by addition of 2-fold molar excess of Sly1p. 

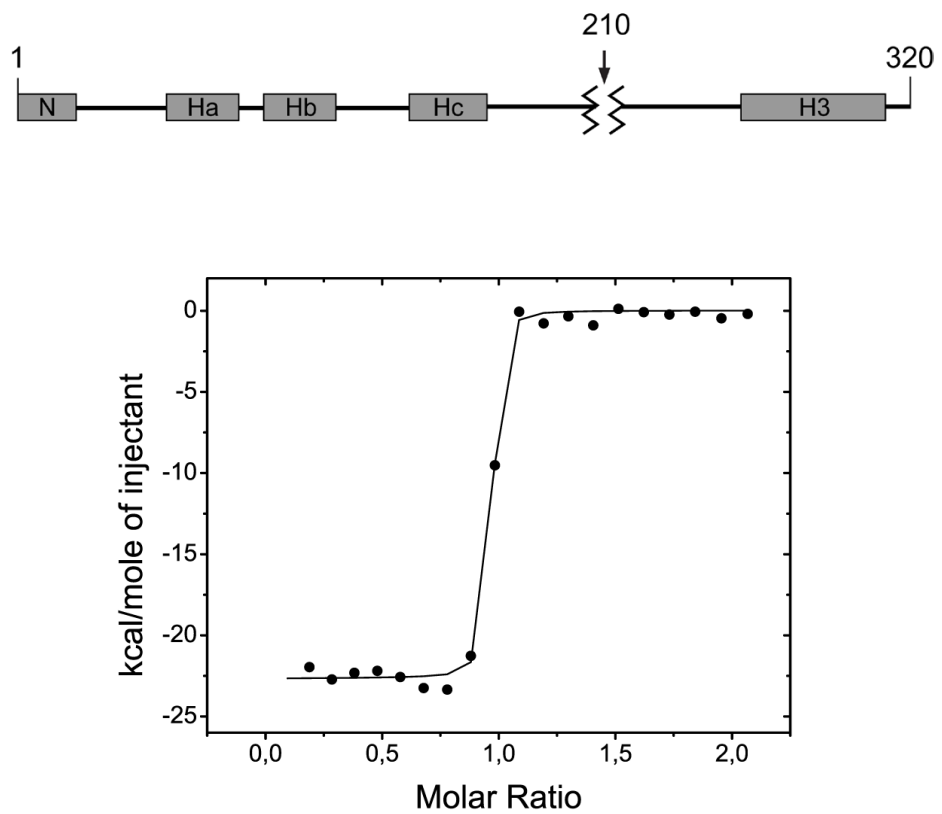

Figure 3.7: Calorimetric titration of Sly1p with the Sed5p (1-210)/ (211-320) complex

A purified complex of Sed5p (1-210) and (211-320) fragments was titrated on Sly1p in an ITC experiment. 15 $\mu \mathrm{M}$ of the protein complex in the syringe was injected into the cell containing $1.7 \mu \mathrm{M}$ Sly $1 \mathrm{p}$. Only the lower panel of the ITC diagram is displayed. The thermodynamic parameters of the reaction are shown in table 3.1. Note that the main difference of the purified complex with respect to wild-type Sed5p is a severed linker. Above the figure, on the schematic drawing, the severed part of the linker is highlighted.

bound molecules were dominated by sharp and narrow peaks, with a small amount of immobile components, indicating no large structural rearrangements of the SNARE motif upon Sly1p interaction. Only slight broadening was observed in some cases (C282, C302), which could be associated with loose tertiary contact. Indeed, it might be possible that only little changes were observed, because the label positions chosen were on a mobile surface, that might not change drastically upon binding of Sly1p. Nevertheless, the results obtained by EPR support the view that the SNARE motif of Sed5p very weakly binds to Sly1p.

In the previous section, I showed that a complex of Sed5p (1-210) and Sed5p (211-320) fragments can be isolated by gel filtration (Figure $3.3 \mathrm{~B}$ ). As this complex contains the SNARE motif as a separate entity, I was wondering whether this complex can bind to Sly1p in a similar manner as wild-type Sed5p. In order to test this using ITC, the complex of Sed5p (1-210) and Sed5p (211-320) fragments was titrated to Sly1p (Figure 3.7). It turned out that both the enthalpy $\left(\Delta \mathrm{N} \approx-23 \mathrm{kcal} /\right.$ mole) and the affinity $\left(\mathrm{K}_{\mathrm{d}} \approx 0.48 \mathrm{nM}\right)$ of the interaction were comparable to the intact Sed5 molecule (Table 3.1). This suggests that 
Sly1p/ Sed5p interaction might remain unaffected, even when the linker between the Habc and SNARE domains of Sed5p is severed. In addition, since the binding enthalpy of the complex is comparable to that of wild-type Sed5p, the separated SNARE motif likely participates in binding.

Since my results demonstrate that the Sed5p N-peptide majorly contributes to Sly1p binding, I also examined the Sly1p/ Sed5p interaction when the N-peptide binding is weakened. The position Phe10 of Sed5p is highly conserved among its homologues and is suggested to be the most important residue for tight binding of the $\mathrm{N}$-peptide region (Yamaguchi et al., 2002, Dulubova et al., 2002, Bracher and Weissenhorn, 2002). Phe10 binds to a highly conserved hydrophobic pocket on Sly1p and it is suggested to have a nucleation role for the helical binding mode of the N-peptide (Bracher and Weissenhorn, 2002). Indeed, previous studies report that the F10A point mutation completely abolishes binding of Sed5p to Sly1p (Yamaguchi et al., 2002, Peng and Gallwitz, 2004). It should be noted, however, that these assessments are based only on qualitative binding assays. Since it might be possible that the methods applied in the previous studies were not able
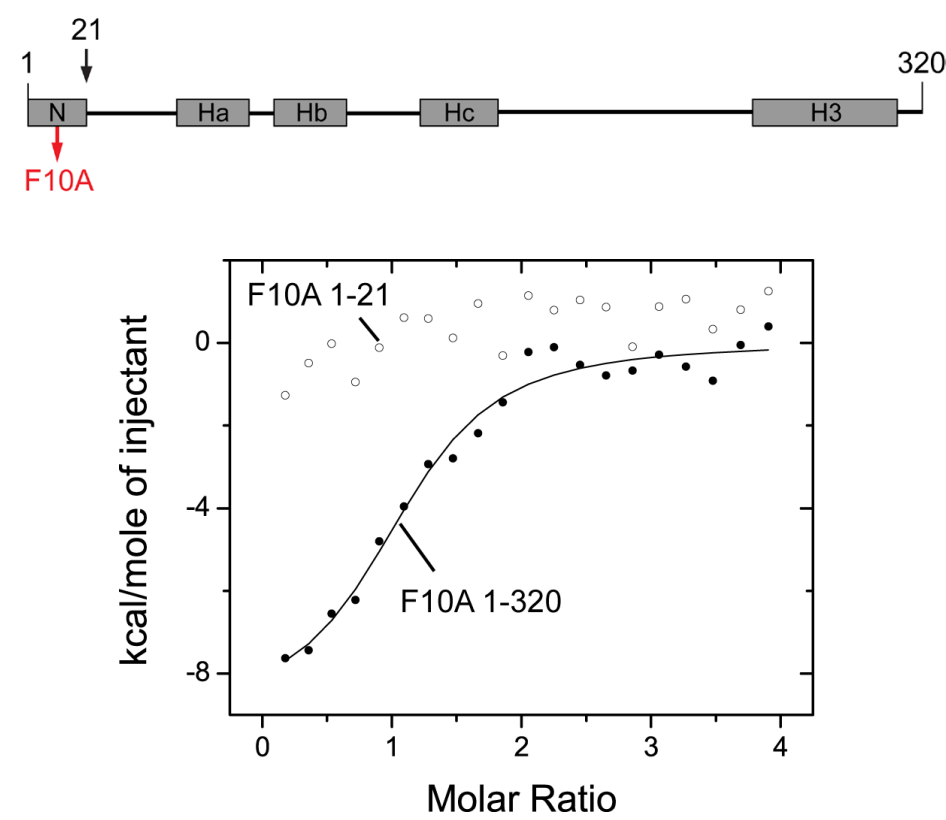

Figure 3.8: Calorimetric titration of Sly1p with Sed5p variants carrying the F10A mutation

Titration curves obtained by ITC experiments, performed between Sly1p and Sed5p F10A mutants (1-21 or 1320). In both experiments, $25 \mu \mathrm{M}$ Sed5p variant in the syringe was injected into the cell containing $1.5 \mu \mathrm{M}$ Sly1p. Only the lower panels of the superimposed ITC diagrams are displayed. The thermodynamic parameters of the reactions are shown in table 3.1. Above the figures, on the schematic drawing, the mutations introduced on Sed5p are marked. 
to detect a weak interaction of the mutated proteins, I re-investigated binding using ITC.

When Sly1p was titrated with the F10A mutant of the sole Sed5p N-peptide (F10A 1-21), no binding was detected (Figure 3.8), supporting previous studies. However, when the F10A mutant of the entire cytosolic portion of Sed5p (F10A 1-320) was used instead, the two proteins interacted, yet with clearly reduced enthalpy $(\Delta \mathrm{H} \approx 9 \mathrm{kcal} / \mathrm{mole})$ and affinity $\left(\mathrm{K}_{\mathrm{d}} \approx 234 \mathrm{nM}\right)$ compared to wild-type (Figure 3.8, Table 3.1). On the one hand, this finding underlines the significance of the N-peptide for the interaction of Sed5p with Sly1p. On the other hand, it reveals that Sed5p is able interact with Sly1p even when Npeptide binding is severely disturbed, supporting the notion that the remaining portion of Sed5p participates in binding as well.

\subsubsection{Does Sly1p bind to the ER-Golgi SNARE complex?}

It has been suggested that Sly1p could function during the SNARE assembly, since it renders Sed5p able to specifically interact with its physiological SNARE partners (Peng and Gallwitz, 2002). This makes it likely that Sly1p is able to interact with the ER-Golgi SNARE complex during or even after its assembly. Indeed, it is currently under debate whether two exocytic SM proteins, the yeast Sec1p and the neuronal Munc18a function on assembled SNARE complexes for executing membrane fusion (Carr et al., 1999, Dulubova et al., 2007, Shen et al., 2007, Deak et al., 2009, Hashizume et al., 2009). Yeast Sec1p is claimed to bind to its cognate syntaxin Sso1p only when it is engaged in a SNARE complex. Munc18a, in contrast, can bind to the closed conformation of Syntaxin 1a, yet it is proposed to bind also to the assembled neuronal SNARE complex, governed by either the N-peptide or the entire N-terminal domain of syntaxin (Misura et al., 2000, Burkhardt et al., 2008, Dulubova et al., 2007). In addition, it has been shown that Munc18a is able to bind, although only very weakly (in the low micromolar range), to the core SNARE complex (Xu et al., 2010).

To test whether Sly1p can bind to a core SNARE complex and whether such an interaction might be controlled by the N-terminal domain of Sed5p, the ER-Golgi SNARE complex was assembled and purified from the cytosolic portions of Sed5p (1-320) and Bet1p (1-118) and the SNARE motifs of Bos1p (151-221) and Sec22p (126-186). ITC revealed that the SNARE complex containing the N-terminal domain of Sed5p is able to bind to Sly1p with 


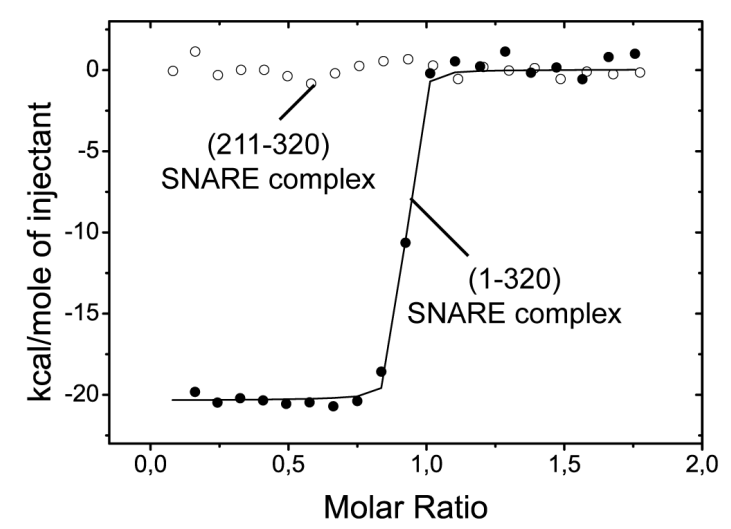

Figure 3.9: Calorimetric titration of Sly1p with ER-Golgi SNARE complexes

SNARE complexes of Sed5p, Bos1p, Bet1p and Sec22p were purified either by using Sed5 (1-320) or (Sed5p

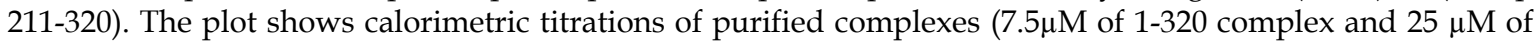
211-320 complex) into Sly1p (1 $\mu \mathrm{M}$ for 1-320 complex and 3.3 $\mu \mathrm{M}$ for 211-320 complex). Only the lower panels of the superimposed ITC diagrams are displayed. The thermodynamic parameters of the reactions are shown in table 3.1.

very high affinity $\left(\mathrm{K}_{\mathrm{d}} \approx 0.23 \mathrm{nM}\right)$ and a favorable enthalpy $(\Delta \mathrm{H} \approx-20.3 \mathrm{kcal} / \mathrm{mole}$ ) (Figure 3.9, Table 3.1). Very likely Sed5p exists in an open conformation when it is part of an assembled SNARE complex. Therefore, interaction of Sly1p with the SNARE complex is, in fact, not very surprising since the N-peptide and the Habc motif of Sed5p are freely available for Sly1p when the SNARE complex is assembled completely. Interestingly, a SNARE complex containing only the SNARE motif of Sed5p (residues 211-320) showed no interaction with Sly1p (Figure 3.9, Table 3.1). This demonstrates that the core region of the SNARE complex does not significantly contribute to binding of Sly1p. Thus, Sly1p most likely interacts with the assembled SNARE complex via the N-peptide and the adjacent region of Sed5p.

\subsubsection{Sly1p interacts with another syntaxin on the retrograde pathway of ER- Golgi transport}

Ufe1p is a yeast syntaxin required for the retrograde vesicular transport between ER and Golgi (Lewis et al., 1997, Patel et al., 1998). Previously, it was reported that Sly1p not only interacts with the N-peptide of Sed5p, but also with that of Ufe1p (Yamaguchi et al., 2002). Since my findings suggest that Sly1p makes use of two binding sites when binding to Sed5p, I also wanted to investigate the Ufe1p interaction in detail. I was able to confirm 


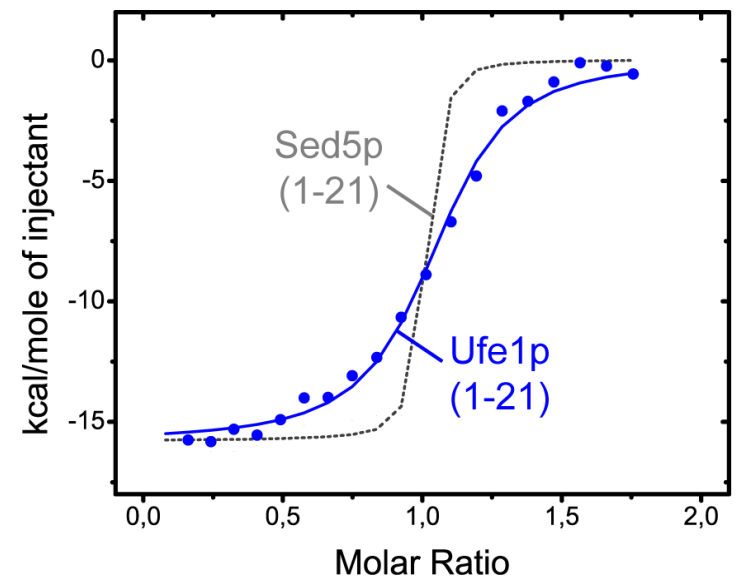

Figure 3.10: Calorimetric titration of Sly1p with Ufe1p N-peptide

The plot shows calorimetric titrations of Sed5p 1-21 $(15 \mu \mathrm{M})$ or Ufe1p 1-21 (15 $\mu \mathrm{M})$ into Sly1p $(2 \mu \mathrm{M})$. The titration curve obtained with Sed5p (1-21) is shown in grey dashed lines, whereas the curve obtained with Ufe1p (1-21) is in blue. Only the lower panels of the superimposed ITC diagrams are displayed. Note that the Sly1p affinity of Ufe1p N-peptide $\left(\mathrm{K}_{\mathrm{d}} \sim 34 \mathrm{nM}\right)$ is much smaller compared to Sed5p N-peptide $\left(\mathrm{K}_{\mathrm{d}} \sim 1.5 \mathrm{nM}\right)$. See table 3.1 for thermodynamic parameters of the reactions.

the Sly1p interaction with the Ufe1p N-peptide (residues 1-21) by using ITC. The enthalpy of the Ufe1p N-peptide binding was comparable to the Sed5p N-peptide $(\Delta \mathrm{H} \approx-16$ $\mathrm{kcal} /$ mole). Nevertheless, the binding affinity of the Ufe1p N-peptide is about 30 -fold lower compared to the Sed5p N-peptide $\left(\mathrm{K}_{\mathrm{d}} \approx 34 \mathrm{nM}\right)$ (Figure 3.10, Table 3.1). The lower affinity of the Ufe1p peptide can perhaps be explained by substitution of some polar residues in Sed5p with the hydrophobic ones in Ufe1p (Yamaguchi et al., 2002, Bracher and Weissenhorn, 2002).

It is possible, like I showed before for Sed5p, that the other regions of Ufe1p do participate in Sly1p binding as well. However, I was unable to purify the entire cytoplasmic domain of Ufe1p in its monomeric form, preventing me from carrying out ITC experiments. Indeed, it has been observed before that, the complete cytosolic portion of Ufe1p forms higher-order multimers in solution (Patel et al., 1998).

Nevertheless, the fact that Sly1p interacts with two different syntaxins, Sed5p and Ufe1p, allowed me to carry out competitive ITC experiments. As mentioned before, $\mathrm{K}_{\mathrm{d}}$ values of Sed5p variants (1-21, 1-210 and 1-320) were in the low-nanomolar range, thus, were too small to be accurately calculated by conventional, non-competitive ITC experiments. On the other hand, the differences in binding affinities between the fragments, according to the other approaches used (in section 3.1.2), must exist. In order to artificially reduce the 


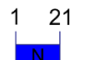

N

$$
\text { Ufe1p }(1-21)+\operatorname{Sed} 5 p(1-320)
$$

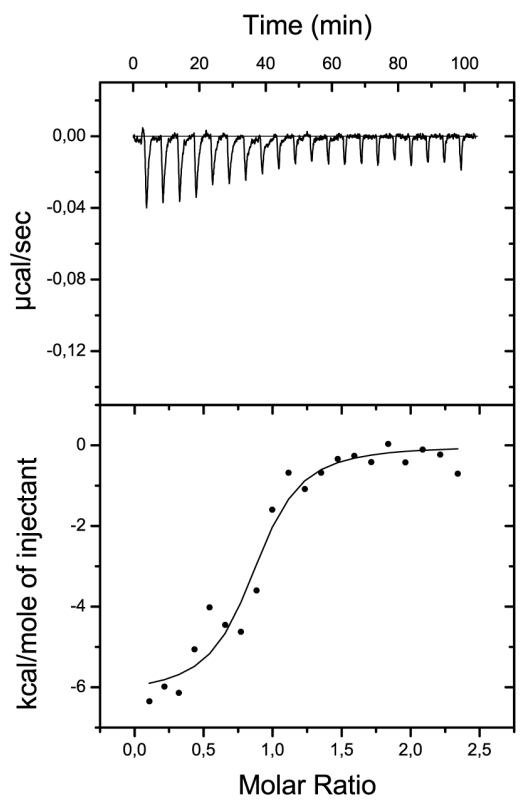

320

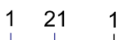

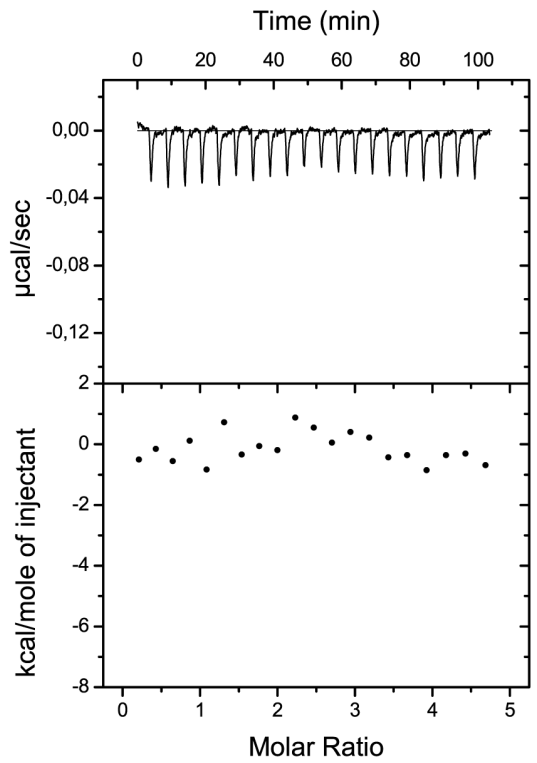

Figure 3.11: Displacement titrations of Sed5p (1-320) and Sed5p (1-210) with Sly1p, using Ufe1p (1-21) as a competitive ligand

The plots show calorimetric titrations of Sed5p 1-320 $(20 \mu \mathrm{M}$, plot on the left) and Sed5p 1-210 (40 $\mu \mathrm{M}$, plot on the right) into Sly1p $(2 \mu \mathrm{M}) /$ Ufe1p 1-21 $(15 \mu \mathrm{M})$ mixes. The experiments were performed as described previously (Velazquez-Campoy and Freire, 2006). In order to do curve-fitting of the competitive binding experiments, the thermodynamic parameters of Sly1p/ Ufe1p 1-21 binding $(\mathrm{N}, \mathrm{Kd}$ and $\Delta \mathrm{H})$ were used as input. Above the figures, on the schematic drawings, the Sed5p and Ufe1p fragments used in the titrations are depicted. See table 3.1 for thermodynamic parameters of the reactions.

binding affinities of the Sed5p fragments, displacement titrations were performed, in which the Sed5p fragments were titrated to a mix of Sly1p and the N-peptide of Ufe1p (121) (Figure 3.11, Table 3.1). The displacement of Ufe1p (1-21) by Sed5p (1-320) was detectable, allowing for determining the binding affinity of Sly1p/ Sed5p (1-320) interaction as $\mathrm{K}_{\mathrm{d}} \approx 0.17 \mathrm{nM}$. This corroborates the high affinity determined by the direct titration approach $\left(\mathrm{K}_{\mathrm{d}} \approx 0.25 \mathrm{nM}\right)$. However, no significant heat change was detected upon the displacement of Ufe1p (1-21) with Sed5p (1-210), possibly because the binding enthalpies of the two ligands for Sly1p were very similar. In other words, the heat uptake associated with Ufe1p (1-21) dissociation was probably fully compensated by the heat release associated with Sed5p (1-210) binding. Thus, the binding affinities of the two fragments of Sed5p could not be compared directly using the displacement approach. 


\subsection{Monitoring assembly of the ER-Golgi SNAREs}

Despite a considerable amount of information available about the cellular function of ERGolgi SNAREs, the biochemical interplay between them is not known in detail. Importantly, the assembly pathways of the SNAREs and the dynamics of their interactions have still not been characterized. Nevertheless, in order to understand the action of regulatory factors, it is important to gain deeper insights into SNARE assembly process.

\subsubsection{Investigating interactions between the ER-Golgi SNAREs by CD spectroscopy}

SNARE proteins are structurally classified as Qa-, Qb-, Qc- and R-SNAREs according to their amino acid contribution to the ionic central layer of the four-helix bundle SNARE complex (Fasshauer et al., 1998b, Kloepper et al., 2007). In ER-Golgi trafficking in baker's yeast Sed5p, Bos1p, Bet1p and Sec22p are believed to function as one unit; Sed5p is the Qa-SNARE or syntaxin, Bos1p is the Qb-, Bet1p is the Qc-, and Sec22p is the R-SNARE. For the much better-studied yeast exocytic SNAREs and SNARE proteins involved in neuronal secretion, the subcomplexes that can form between SNARE motifs prior to SNARE assembly have been already identified, whereas they are unknown for the ERGolgi SNAREs. Interestingly, for both secretory SNAREs, assembly in vitro is proposed to proceed through a partially helical Qabc-intermediate (Fasshauer and Margittai, 2004, Fasshauer et al., 1997a, Fasshauer et al., 2002, Fiebig et al., 1999, Nicholson et al., 1998). This raises the question whether a similar subcomplex of Qabc-SNAREs might act as an intermediate for the assembly of ER- Golgi SNAREs. Or could there be a different assembly route providing rapid SNARE assembly for SNARE proteins functioning in an intracellular trafficking step? To address these questions, I initially examined which stable subcomplexes can form between ER-Golgi SNAREs.

Generally, in order to perform CD experiments, I purified the protein segments spanning the SNARE motifs: Sed5p (QaH3, residues 211-320), Bos1p (Qb, residues 151-221) and Sec22p (R, residues 126-186). However, I used the entire cytosolic portion of Bet1p (Qc, residues 1-118), since the protein was unstable in the absence of its N-terminal extension of the SNARE domain (Figure 3.12 A). At first, I measured the far-UV CD spectra of 


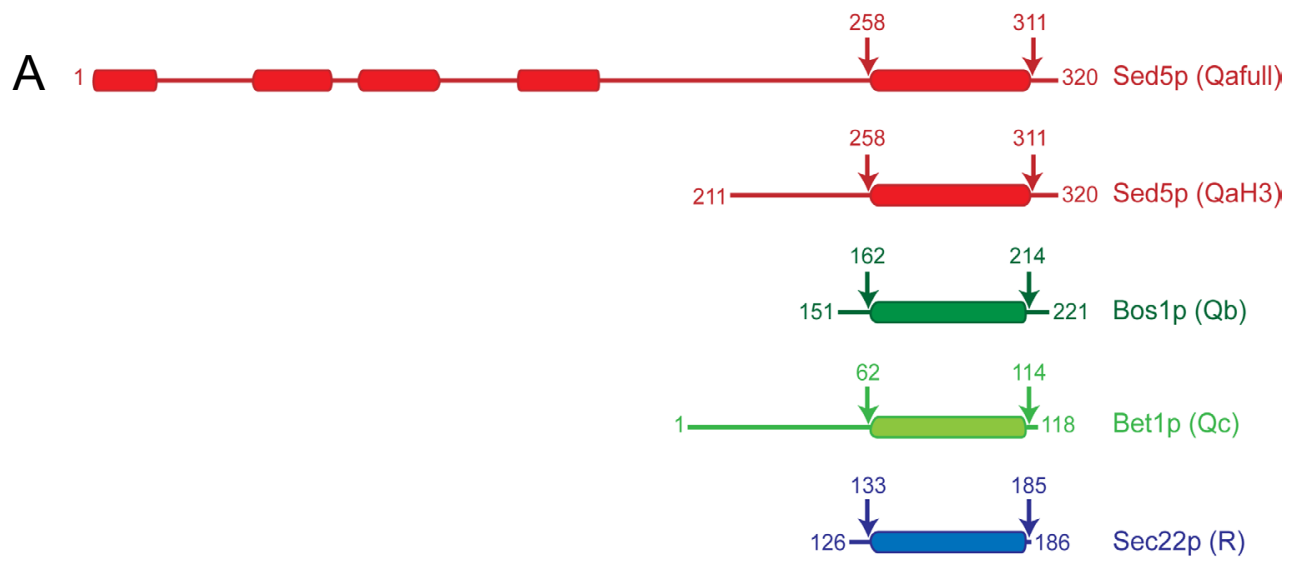

B

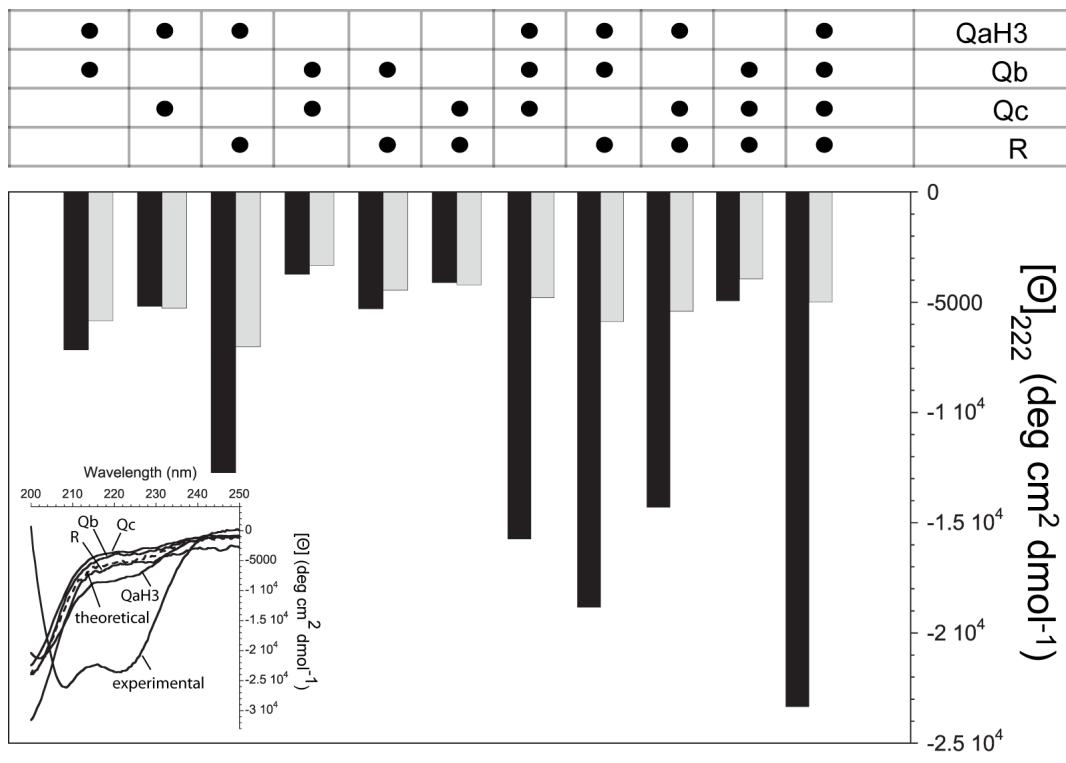

Figure 3.12: Interaction of ER-Golgi SNAREs observed by CD spectroscopy

A. Schematic drawing of the SNARE segments used in the CD experiments. Sed5p (QaH3 and Qafull) is displayed in red, Bos1p $(\mathrm{Qb})$ is in dark green, Bet1p $(\mathrm{Qc})$ is in light green and Sec22p $(\mathrm{R})$ is in blue. The coreSNARE regions predicted from the SNARE database (Klöpper et al., 2007) are shown in rounded boxes and the boundary residues are highlighted by arrows. B. Putative complexes which could form between ER-Golgi SNAREs were observed by CD spectroscopy. CD spectra were collected either from single SNARE segments $(5 \mu \mathrm{M})$ or after their overnight incubation in equimolar amounts. As an example, inset shows CD spectra of individual SNAREs and the spectrum obtained from a combination of all. Theoretical noninteracting spectrum, calculated from the observed CD spectra of the SNARE segments, is displayed in the dashed lines. In the bar diagram, the black columns represent the mean residue ellipticity values of various SNARE combinations. In addition, the mean residue ellipticity values calculated from the theoretical noninteracting spectra are shown in grey columns. All experiments were performed at room temperature in $0.1 \mathrm{~cm}$ cuvettes, in a buffer containing $20 \mathrm{mM} \mathrm{NaH}{ }_{2} \mathrm{PO}_{4} / \mathrm{Na}_{2} \mathrm{HPO}_{4} \mathrm{pH}$ : 7.4 and $200 \mathrm{mM} \mathrm{NaCl}$. 
individual SNAREs. Similar to what has been observed for other SNARE motifs (Zwilling et al., 2007, Fasshauer et al., 1998a, Antonin et al., 2000, Rice et al., 1997), invidiual ERGolgi SNAREs were largely unstructured. In order to detect stable complexes formed between the ER-Golgi SNAREs, I mixed and incubated the SNARE motifs in all combinations and then collected their far-UV CD spectra (Figure $3.12 \mathrm{~B}$ ). Combination of Sed5p (QaH3) and Sec22p (R) led to an increase in a-helical content, yet there were no detectable binary interactions between the other SNAREs.

Large structural changes only occurred upon mixing three SNARE motifs. Three out of four putative ternary complexes were observed by CD spectroscopy. Interestingly, all three included the SNARE motif of Sed5p (Figure $3.12 \mathrm{~B}$ ). As my later experiments showed, the ternary mixes of Qabc-, QabR- and QacR-SNAREs form stable complexes, because it was possible to purify them chromatographically (Appendix A.2). Of note, in a previous study, it was found that the neuronal Qabc-, QabR- and QacR-SNAREs can also form stable assemblies (Fasshauer et al., 1998a), whereas, comparable to the ER-Golgi

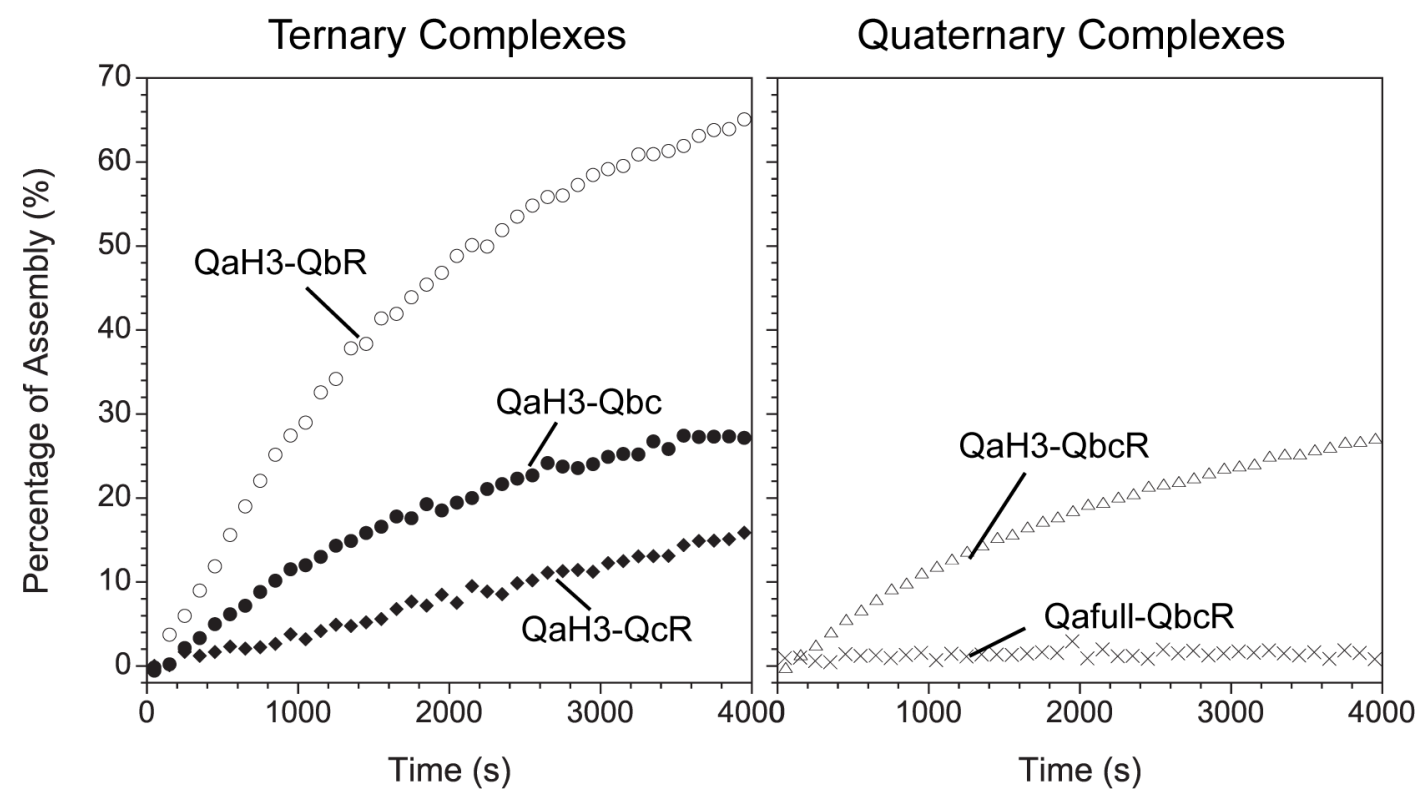

Figure 3.13: Assembly kinetics of complexes formed between ER-Golgi SNAREs, followed by CD spectroscopy

Assembly kinetics of various complexes formed between ER-Golgi SNAREs were observed by monitoring the change in the mean residue ellipticity at $222 \mathrm{~nm}$ over time. The mean residue ellipticities of stable protein complexes were measured after the overnight incubation of the SNARE components and the assembly kinetics was normalized accordingly. Each measurement was started after final addition of $2 \mu \mathrm{M}$ Qa-SNARE (Sed5p) to an equimolar mixture of other SNAREs. All experiments were performed at room temperature in 1 cm cuvettes, in a buffer containing $20 \mathrm{mM} \mathrm{NaH}{ }_{2} \mathrm{PO}_{4} / \mathrm{Na}_{2} \mathrm{HPO}_{4} \mathrm{pH}: 7.4$ and $200 \mathrm{mM} \mathrm{NaCl}$. 
SNAREs, no stable complex was found for a mix that did not contain the Qa-SNARE.

I additionally monitored the assembly kinetics of the ternary complexes by CD spectroscopy. For this, I mixed the SNARE segments and followed the change of the CD signal at 222nm over time (Figure 3.13). The assembly of all complexes was relatively slow (on average $\leq 50 \%$ of assembly occurred within an hour, when $2 \mu \mathrm{M}$ SNAREs were used), interestingly assembly of the QabR complex was faster than of the other mixtures. The assembly kinetics of the quaternary SNARE complex was also followed in a similar manner either using the SNARE motif (QaH3: residues 211-320) or the entire cytosolic portion of Sed5p (Qafull: residues 1-320) (Figure 3.13). Assembly was slow when the QaH3 was used, in comparable speed to the ternary complexes. On the other hand, in the presence of Qafull, no structural change was observed, suggesting that the quaternary complex did not form within the time of the experiment (about one hour). In the previous section (section 3.1.1), I have shown that individual Sed5p adopts a closed conformation, in which the Habc and the H3 domains of the syntaxin interact. For other syntaxins, Syntaxin 1a and Sso1p, such a closed conformation has been shown to interfere with the SNARE assembly speed of the bound H3 domain. As full-length Sed5 apparently formed a SNARE complex much more slowly than its isolated H3 motif, it is likely that the closed conformation of Sed5p also interferes with its $\mathrm{H} 3$ motif accessibility for its SNARE partners and as a result retards SNARE assembly.

\subsubsection{Monitoring assembly kinetics of the ER-Golgi SNAREs by fluorescence anisotropy}

Experiments employing CD spectroscopy provided me with a list of potential intermediates of the SNARE assembly reaction. As shown in the previous section, since the SNARE motifs are largely unstructured, the structural rearrangements upon their folding can be easily followed by $\mathrm{CD}$ spectroscopy. Because of the nature of $\mathrm{CD}$ spectroscopy, it is problematic to add stoichiometric amounts of already folded proteins or domains to this reaction. In fact, already the addition of the alpha-helical Habc-domain to the SNARE assembly reaction decreased the percentage of the observable structural change. For this reason, it was not possible to monitor the effect of the large SM protein Sly1p on the SNARE assembly reaction using CD spectroscopy. Therefore, to be able to determine the role of Sly1p during assembly of the ER-Golgi SNAREs, a different read-out 

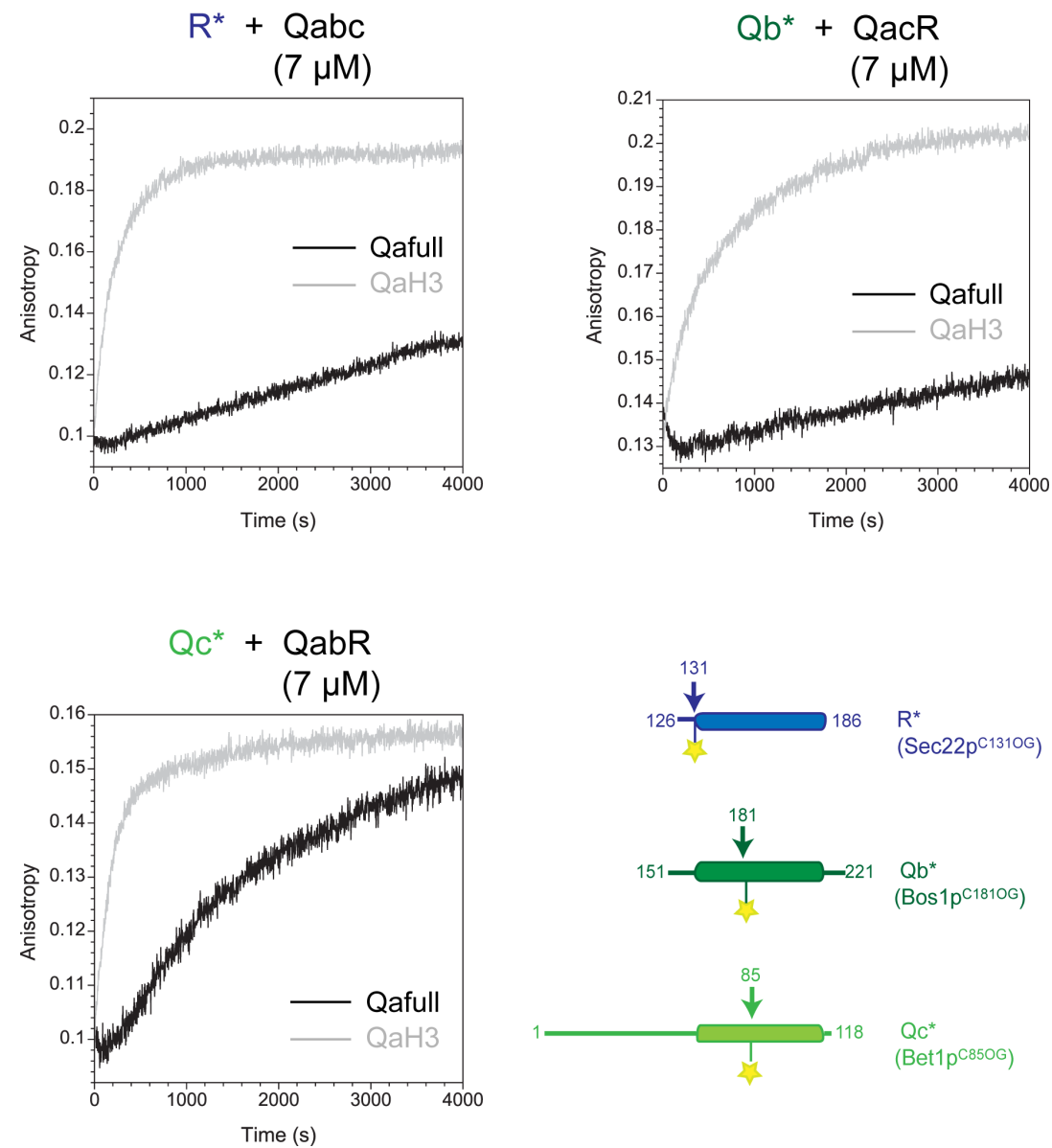

Figure 3.14: Assembly kinetics of ER-Golgi SNAREs observed by fluorescence anisotropy

SNARE assembly was monitored by the increase in the anisotropy of $\sim 400 \mathrm{nM}$ fluorescent $\mathrm{Qb}^{*}$ - (Bos1p C181OG), $\mathrm{Qc}^{*}$ - (Bet1p C85-OG) or R*-SNAREs (Sec22p C131-OG) upon mixing them with $7 \mu \mathrm{M}$ of their partners. The fluorescent Sec22p C131-OG was preferably used, but not Sec22p C180-OG, since the reactions were slightly slower in the latter case (data not shown). The schematic drawing of the fluorescent SNAREs are shown next to the plots. Either QaH3 or Qafull were included in the assembly reactions, shown in grey curves and in black curves, respectively. Each measurement was started immediately after mixing the components. All experiments were performed at room temperature in a buffer containing $20 \mathrm{mM} \mathrm{Na} \mathrm{H}_{2} \mathrm{PO}_{4} / \mathrm{Na}_{2} \mathrm{HPO}_{4} \mathrm{pH}$ : 7.4 and $200 \mathrm{mM} \mathrm{NaCl}$. Since the reaction volume was $180 \mu \mathrm{l}$, each experiment was performed in an ultramicro fluorescence cell.

needed to be developed. In previous studies, fluorescence anisotropy was extensively used in order to follow the dynamics and the regulation of neuronal SNAREs (Fasshauer and Margittai, 2004, Pobbati et al., 2006, Burkhardt et al., 2008). Thus, I chose to develop tools to monitor the assembly of ER-Golgi SNAREs by fluorescence anisotropy.

In order to perform anisotropy experiments, fluorophores needed to be attached at specific sites in the SNARE proteins. Since the ER-Golgi SNAREs do not have cysteines in their native form, substitutions of single amino acid residues with cysteines for specific labeling were performed by site-directed mutagenesis. Positions for mutagenesis were 
carefully selected in order not to interfere with the SNARE assembly reaction. Since the crystal structure of ER-Golgi SNARE complex is unknown, the information available at the SNARE database (http:/ / bioinformatics.mpibpc.mpg.de/snare) (Kloepper et al., 2007) was used to predict the residues buried inside SNARE bundle, i.e. the ones that form the hydrophobic layers. Positions that are predicted to reside on the helix surface of the SNARE complex were selected for modification. For the different SNAREs, the following positions were mutated to cysteines: Bos1p (151-221 E186C), Bet1p (1-118 G85C) and Sec22p (126-186, D131C and K180C). The purified proteins were labeled with Oregon Green 488 iodoacetamide. Sed5p SNARE motif was left unlabeled in all anisotropy assays.

First, I followed the speed of the four-helix bundle SNARE complex, using the sole SNARE motifs. For this, the fluorescently labeled Bos1p (Bos1p 151-221 C181-OG, termed $\mathrm{Qb}^{*}$ ) or Bet1p (Bet1p 1-118 C85-OG, termed $\mathrm{Qc}^{*}$ ) or Sec22p (Sec22p 126-186 C131-OG, termed $R^{*}$ ) were each mixed with their respective partner SNAREs (Figure 3.14). The increase in the fluorescence anisotropy over time was indicative of complex formation. In agreement with my previous observation, SNARE assembly occurred rather slowly, such that I needed to add up to $\approx 7 \mu \mathrm{M}$ of unlabeled SNAREs into each reaction in order to complete the reaction within $\approx 1.5$ hours (Figure 3.14, grey curves). I noted that comparable speed of assembly was observed for the neuronal SNAREs when only $\approx 0.5$ $\mu \mathrm{M}$ of unlabeled proteins were added (Burkhardt et al., 2008), suggesting that the yeast ER-Golgi SNAREs assemble much more slowly than their neuronal homologues.

The same approach was used to measure the assembly kinetics of the full-length Sed5p. As expected, SNARE assembly was even slower when the entire cytosolic portion of Sed5p was used in comparison to when the sole SNARE motif of Sed5p was used (Figure 3.14, black curves). This is in agreement with the results obtained by CD spectroscopy (see Figure 3.13). Hence, this finding supports the notion that Sed5p preferentially adopts a closed conformation that interferes with the SNARE complex formation.

\subsubsection{Qabc subcomplex of ER-Golgi SNAREs can be stabilized to serve as an assembly intermediate for SNARE complex formation}

As mentioned before, a subcomplex formed between Syntaxin1a and SNAP-25 is thought to act as an intermediate for the neuronal SNARE assembly (Fasshauer et al., 1997, 
Fasshauer et al., 2002, Fasshauer and Margittai, 2004). On the other hand, in vitro, Syntaxin1a and SNAP-25 readily form a remarkably stable four-helical bundle in 2:1 stoichiometry (Fasshauer et al., 1997). The second syntaxin of this complex is believed to occlude the Synaptobrevin binding site (Margittai et al., 2001, Xiao et al., 2001). Thus, in vitro assembly of the neuronal SNAREs is slow since most likely, Synaptobrevin competes with another syntaxin to bind to the transient 1:1 heterodimer of Syntaxin1a and SNAP-25 (Fasshauer and Margittai, 2004). On the other hand, the neuronal SNARE assembly was drastically accelerated when the 1:1 Syntaxin/ SNAP-25 complex was artificially stabilized using a C-terminal fragment of synaptobrevin (Syb). When the complex contained the following synaptobrevin fragments (Syb42-96, Syb49-96 or Syb60-96) rapid binding of synaptobrevin and displacement of the short fragment was observed using fluorescence anisotropy as read-out (Pobbati et al., 2006).

The $\mathrm{CD}$ and the fluorescence anisotropy experiments described in the above sections showed that in vitro assembly of the ER-Golgi SNAREs is also slow, indeed, even slower than the assembly of their neuronal homologues. The CD experiments suggested that for the ER-Golgi-SNAREs a subcomplex between Qa-, Qb- and Qc-SNAREs, analogous to the neuronal SNAREs, can form. Indeed, the Qabc complex of the ER-Golgi-SNAREs can be purified as a stable entity. As shown in my Master thesis, however, preforming the Qabc complex does not accelerate SNARE complex formation (Demircioglu, 2008 p.45-47). The stoichiometry and the configuration of the ER-Golgi Qabc complex is unknown and it cannot be ruled out that the R-SNARE binding site is obstructed like for the neuronal Qabc complex. Therefore, I wanted to find out next whether the ER-Golgi Qabc complex can serve as an acceptor for rapid SNARE assembly, when it is stabilized in a similar manner as the neuronal Qabc-complex.

The aforementioned Synaptobrevin fragments that were used to stabilize the 1:1 syntaxin/SNAP-25 complex were truncated on the N-terminal side of the heptad repeat layers: -4 (i.e. Syb42-96), -2 (i.e. Syb49-96), or +1 (i.e. Syb60-96) (Pobbati et al., 2006). In order to form an analogous acceptor complex of the ER-Golgi SNAREs, I firstly attempted to purify a Sed5p/ Bos1p/ Bet1p (Qabc) complex containing the Sec22p fragment (150186), which is truncated on the N-terminal side of the -2 layer, equivalent to Syb49-96. The SNARE motif of Sed5p was used for complex formation. However, no stable complex containing this Sec22 fragment could be isolated, probably because the fragment was too short. Indeed, I was then able to purify a stable Sed5p/ Bos1p/ Bet1p (Qabc) complex 

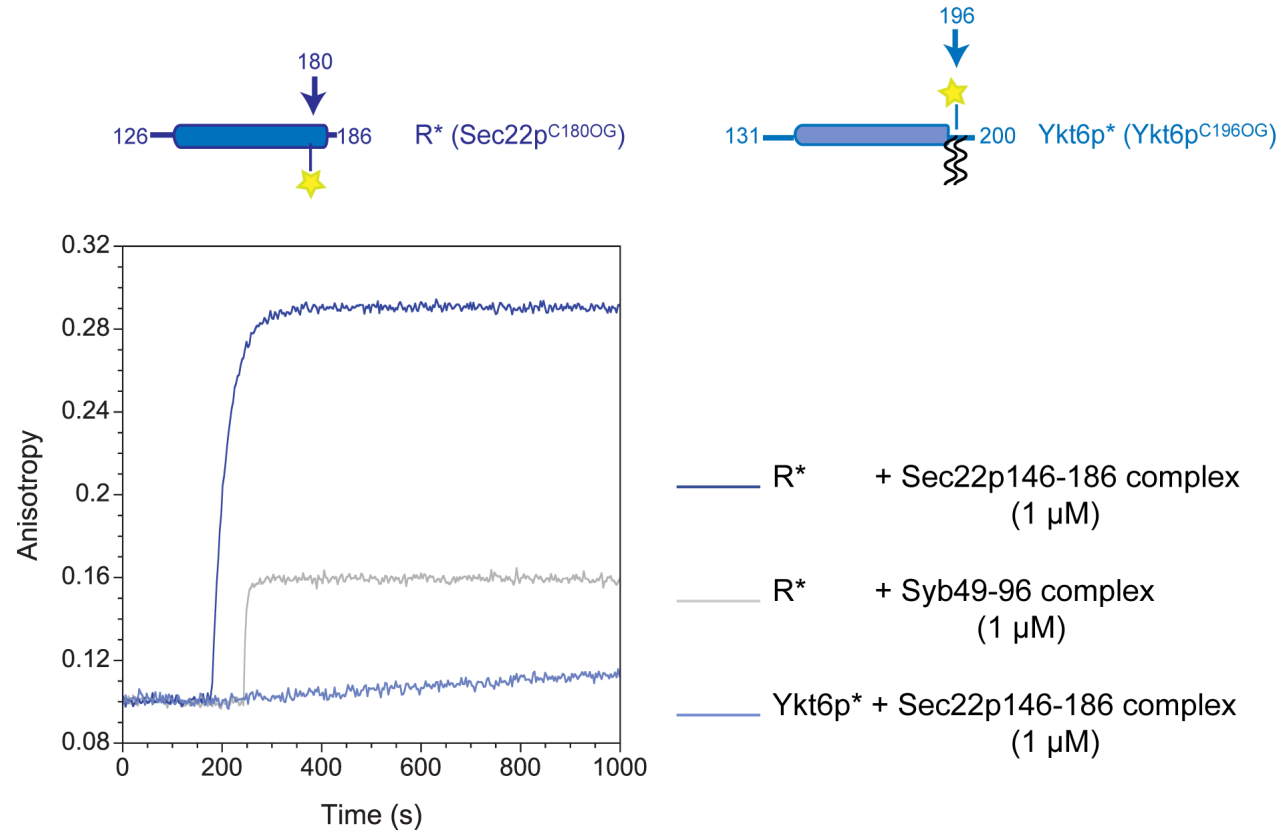

$R^{*} \quad+$ Sec22p146-186 complex

$(1 \mu \mathrm{M})$

$R^{*} \quad+$ Syb49-96 complex

$(1 \mu \mathrm{M})$

Ykt6p* + Sec22p146-186 complex

$(1 \mu \mathrm{M})$

Figure 3.15: A stabilized Qabc-complex of ER-Golgi SNAREs can serve as acceptor for Sec22p.

SNARE assembly was monitored by the increase in the anisotropy of $\sim 50 \mathrm{nM}$ fluorescent $\mathrm{R}^{*}$ (Sec22p C180OG) or Ykt6p* (Ykt6p C196-OG) upon mixing them with $\sim 1 \mu \mathrm{M}$ of stabilized acceptor complexes. The schematic drawing of the fluorescent SNAREs are shown on top of the plot. The stabilized ER-Golgi acceptor complex was containing Sed5p (211-320), Bos1p (151-221), Bet1p (1-118) and Sec22p (146-186). The neuronal acceptor complex was kindly provided by Katrin Wiederhold and was formed of SNARE motifs of Syntaxin $1 \mathrm{a}$ and SNAP-25 and Syb (49-96) fragment. Each measurement was started in the presence of sole R* or Ykt6p* and the additional components were added after. All experiments were performed at room temperature, in a total reaction volume of $1200 \mu \mathrm{l}$ and in a buffer containing $20 \mathrm{mM} \mathrm{NaH} \mathrm{PO}_{4} / \mathrm{Na}_{2} \mathrm{HPO}_{4} \mathrm{pH}$ : 7.4 and $200 \mathrm{mM}$ $\mathrm{NaCl}$.

containing the slightly longer Sec22p fragment (residues 143-186, until layer -4). It turned out, however, that fluorescent Sec22p was unable to bind to this complex.

As an additional attempt, the Sed5p/ Bos1p/ Bet1p complexes were purified together with a Sec22p fragment of an intermediate size, i.e. Sec22p (146-186) fragment that lacked the residues N-terminally of the heptad repeat layer -3 (analogous to a Syb45-96 fragment). For this complex, rapid displacement of the Sec $22 p$ fragment and binding of the fluorescent, full-size Sec22p was observed by fluorescence anisotropy (Figure 3.15). This suggests that an artificially stabilized Qabc complex of ER-Golgi SNAREs enables fast SNARE assembly, similar to its neuronal counterpart. Interestingly, Sec22p could also bind rapidly to a stabilized neuronal acceptor complex (Figure 3.15).

As mentioned in introduction, the function of Sec $22 p$ can be substituted by another RSNARE, Ykt6p, during anterograde ER-Golgi transport (Liu and Barlowe, 2002). Can 
Ykt6p bind to the stabilized Qabc complexes like Sec22p? In order to test this, a portion of Ykt6p (residues 131-200) spanning the SNARE motif was cloned. The native Ykt6p has two Cysteines at the C-terminal positions 196 and 197, which are palmitoylated in vivo. To obtain a Ykt6p construct that possess only one cysteine residue, one of the native cysteines, residue 197, was substituted with a Serine by site-directed mutagenesis. Interestingly, fluorescent Ykt6p (Ykt6p 131-200 C196-OG) bound only very slowly to the stabilized ER-Golgi Qabc-complex (Figure 3.15). This suggests that the two R-SNAREs Sec22 $p$ and $Y k t 6 p$ differ in their SNARE binding properties.

\subsubsection{A transient QabR subcomplex allows rapid assembly of the ER-Golgi SNARES}

Prior to applying a strategy to stabilize the Qabc complex of neuronal SNAREs via an RSNARE fragment, it had been already shown that a transient Qabc intermediate can serve as a fast binding site for synaptobrevin. This transient Qabc intermediate was formed upon premixing Syntaxin 1a with an excess amount of SNAP-25, whereas increasing amounts of Syntaxin 1a rather slowed down the SNARE assembly. It was therefore proposed that an excess of SNAP-25 promoted formation of a transient 1:1 Qabc transient intermediate (Fasshauer and Margittai, 2004).

As demonstrated in the previous section, the Qabc subcomplex of ER-Golgi SNAREs allows rapid SNARE assembly when stabilized by a Sec22p fragment. On the other hand, it is still a question whether the Qabc subcomplex can serve as an intermediate for SNARE complex formation in vivo. Indeed, it has not been investigated so far whether assembly of the ER-Golgi SNAREs requires a transient intermediate. As shown in section 3.2.1, CD spectroscopy experiments indicated that three different subcomplexes, each containing only three different SNARE subunits, can form between the ER-Golgi SNAREs: Qabc, QabR, and QacR. This raises the question whether one of those subcomplexes serves as a transient assembly intermediate. Since previously, a premixing strategy was employed to obtain a transient intermediate of neuronal SNAREs, I decided to use a similar approach for the ER-Golgi SNAREs.

In section 3.2.2, to follow the SNARE assembly, the fluorescent SNAREs (Qb*, $\mathrm{Qc}^{*}$ or $\left.\mathrm{R}^{*}\right)$ were mixed with their respective unlabeled SNARE partners. In order to enhance 
formation of the individual subcomplexes prior to assembly of the four-helix bundle SNARE assembly, I pre-mixed the unlabeled SNAREs for several hours before adding the fluorescent SNARE. In the initial experiments, I pre-mixed equimolar amounts of SNARE subunits, because the stoichiometry of the Qabc, QabR and QacR complexes were unknown.

First, I pre-incubated Qa-, Qb-, and Qc-SNAREs, before mixing them with the fluorescent $\mathrm{R}^{*}$ in order to start the SNARE assembly process. Assembly kinetics were then compared to those in which Qa-, Qb- and Qc-SNAREs were mixed without pre-incubation. As can be seen in figure 3.16 A, pre-incubation did not enhance the assembly rate of the ER-Golgi SNARE complex. Remarkably, R* binding was even somewhat slower when the QaH3-, Qb-and Qc-SNAREs were allowed to preassemble. Also no acceleration was observed when the entire cytoplasmic region of Sed5p, Qafull, was used instead of the SNARE motif to preform the Qabc complex, although the entire reaction was much slower compared to the one using the SNARE motif of Sed5p, QaH3. Therefore, a subcomplex formed of Qa-, Qb- and Qc-SNAREs does not seem to be able to provide a site for fast Sec22p binding.

Again, no significant acceleration was observed when the Qa- (QaH3 or Qafull), Qc- and R-SNAREs were preincubated and mixed with $\mathrm{Qb}^{*}$, suggesting that the QacR subcomplex also cannot serve as a fast binding site for SNARE complex formation (Figure $3.16 \mathrm{~B}$ ). Remarkably, mixing of labeled Bet1p, Qc*, with a premix of Qa-, Qb- and R-SNAREs led to a drastic increase in the rate of SNARE complex formation, regardless whether the QaH3 or Qafull was used for preassembly (Figure $3.16 \mathrm{C}$ ). When the premixes were added to the reactions at $7 \mu \mathrm{M}$, the reactions were too fast for the manual mixing approach, whereas addition of premixes at $2.7 \mu \mathrm{M}$ allowed for visualizing the rapid assembly reaction. This suggests that the Qa-, Qb- and R-SNAREs can assemble into a complex that allows for rapid binding of Bet1p. Formation of an active sub-complex, i.e. a complex that can serve as a fast binding site, seems to require the presence of all three SNAREs: Qa, Qb and R. Indeed, when only Qa- and Qb- or Qa- and R-SNAREs were premixed, the SNARE assembly was still slow, i.e. in comparable speed to the reactions performed without pre-mixing (data not shown).

These experiments shown above demonstrate that an active assembly intermediate for SNARE complex formation can be formed between Qa-, Qb- and R-SNAREs. However, it 
A

$\mathrm{R}^{*}+$ Qabc

$(7 \mu \mathrm{M})$

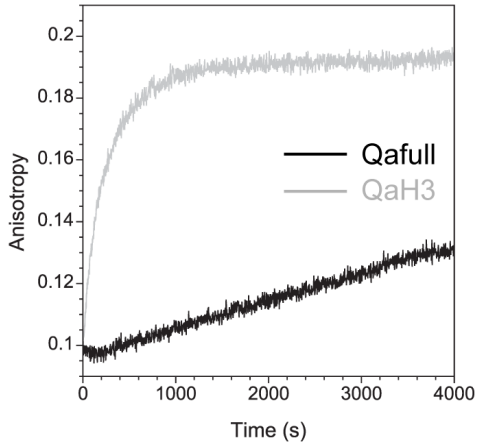

B

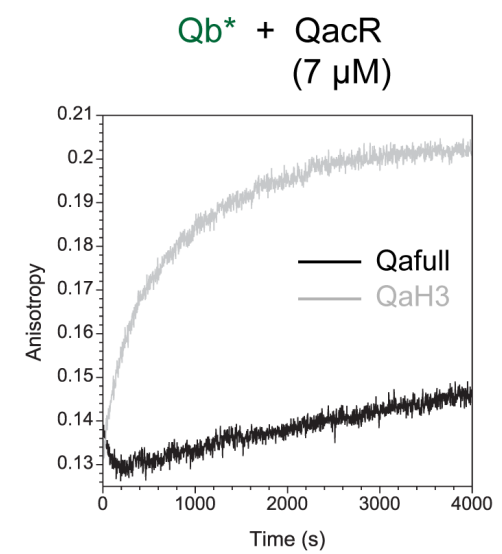

$R^{*}+$ Qabc PRE-MIXED

$(7 \mu \mathrm{M})$

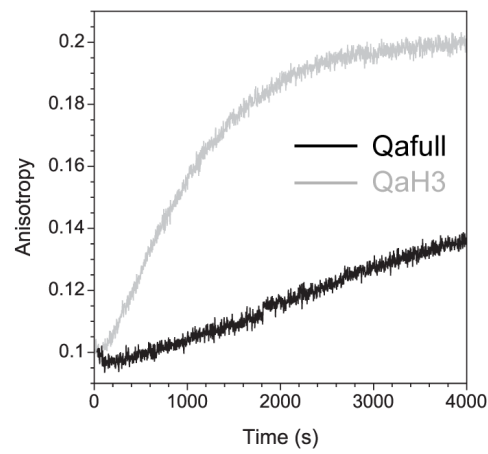

$\mathrm{Qb}^{*}+$ QacR PRE-MIXED

$(7 \mu \mathrm{M})$

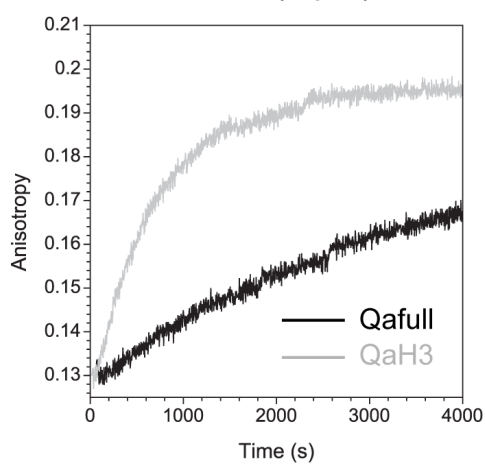

C $\quad Q^{*}+Q a b R$ $(2.7 \mu \mathrm{M})$

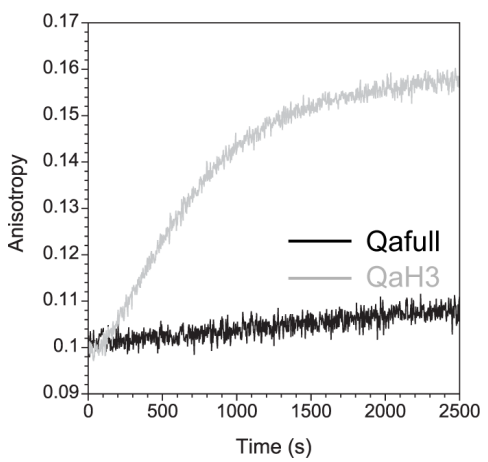

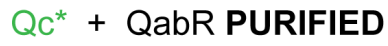

$(2.7 \mu \mathrm{M})$

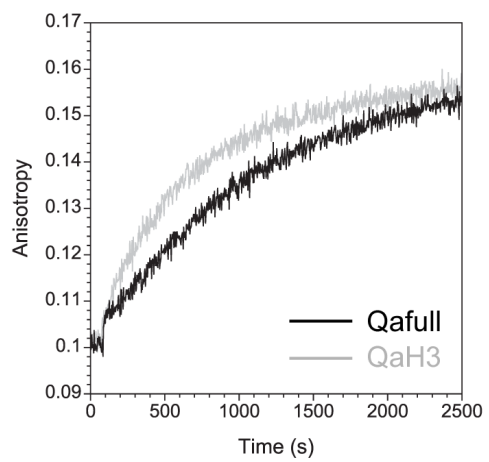

$\mathrm{Qc}^{*}+$ QabR PRE-MIXED

$(2.7 \mu \mathrm{M})$

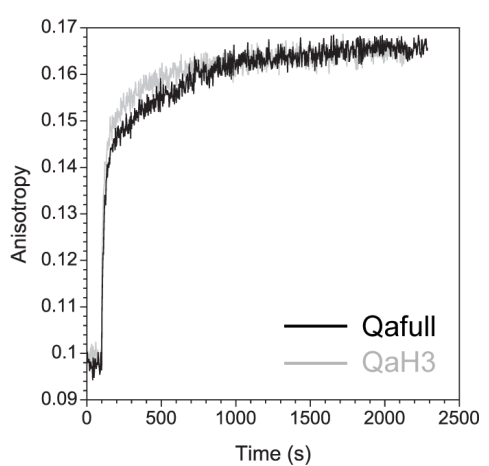

Figure 3.16: Assembly kinetics of ER-Golgi SNAREs, observed after pre-incubation of unlabeled SNAREs

A, B. SNARE assembly was monitored by the increase in the anisotropy of $\sim 400 \mathrm{nM}$ fluorescent Qb*- (Bos1p C181-OG) or R*-SNAREs (Sec22p C131-OG) upon mixing them with their partners. Either QaH3 or Qafull were included in the assembly reactions, shown in grey curves and in black curves, respectively. The plots on the left show the reactions where $\sim 7 \mu \mathrm{M}$ of unlabeled SNAREs were immediately mixed with the $\sim 400 \mathrm{nM}$ labeled SNARE. The unlabeled SNAREs were pre-incubated overnight and added at $\sim 7 \mu \mathrm{M}$ in the cuvettes to obtain the plots on the right. All experiments were performed at room temperature, in a total reaction volume of $180 \mu \mathrm{l}$. 
C. Similarly, SNARE assembly was monitored by the increase in the anisotropy of $\sim 50 \mathrm{nM}$ fluorescent $\mathrm{Qc}^{*}$ SNARE (Bet1p C85-OG) upon mixing it with its partners. Either Qafull or QaH3 were used in each experiment. Unlabeled SNAREs were added either without pre-incubation (plot on the left) or as purified complexes (plot in the middle) or after pre-incubation overnight (plot on the right). The premix solutions contained $\sim 11 \mu \mathrm{M}$ of QabR-SNAREs. Each measurement was started in the presence of sole $\mathrm{Qc}^{*}$ and the additional components were added after, up to $\sim 2.7 \mu \mathrm{M}$. All experiments were performed at room temperature, in a total reaction volume of $1300 \mu 1$.

is unclear whether the active intermediate is a stable entity or a transient assembly. In order to test this, I assembled the QabR sub-complex and separated it from the monomers by ion-exchange chromatography. Two different QabR complexes were formed by using either QaH3 or Qafull. When $\approx 2.7 \mu \mathrm{M}$ of the QabR complexes were mixed with labeled Bet1p, the reactions were clearly slower than when the premix was used (Figure $3.16 \mathrm{C}$ ). This strongly suggests that the acceptor complex obtained by pre-mixing is not a stable entity, but most likely exists in a transient state, whereas the QabR complex that can be purified might be a stable, off-pathway product in which, like in the 2:1 neuronal syntaxin-SNAP-25 complex, the fourth binding site is obstructed. Notably, it did not matter for the speed of SNARE complex formation whether the purified QabR subcomplex contained QaH3 or Qafull. This suggests that both types of QabR subcomplexes probably offer the same binding site and that the presence of the $\mathrm{N}$ terminal part of the Sed5p molecule does not interfere with binding of Bet1p.

The transient nature of the active QabR assembly intermediate suggests that it probably exists in a dynamic equilibrium with its constituents. In order to gain more insights into its state, I performed additional assays at different concentrations. When I overnight preincubated $\approx 11 \mu \mathrm{M}$ of Qa-, Qb- and R-SNAREs, and diluted them up to $\approx 2.7 \mu \mathrm{M}$, or $\approx$ $1 \mu \mathrm{M}$, or $\approx 0.5 \mu \mathrm{M}$ before mixing them with the fluorescent $\mathrm{Qc}^{*}$ (Figure 3.17); in all reactions, an initial rapid increase in fluorescence anisotropy was observed, indicating rapid binding of Bet1p. This shows that the transient intermediate forms at different concentrations. However, at lower concentrations a slower, second reaction phase was more prominent, suggesting that at lower concentrations less of the active assembly intermediate was formed. Thus, the transient QabR assembly, indeed, exists in a dynamic equilibrium with its components. Interestingly, QabR mixes containing the Qafull were less stable compared to the mixes containing the QaH3. 
$\mathrm{QC}^{*}+$ QabR PRE-MIXED

$(2.7 \mu \mathrm{M})$

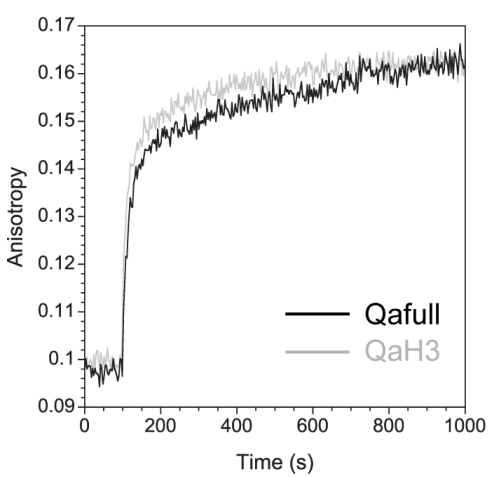

$Q^{*}+$ QabR PRE-MIXED

$(1 \mu \mathrm{M})$

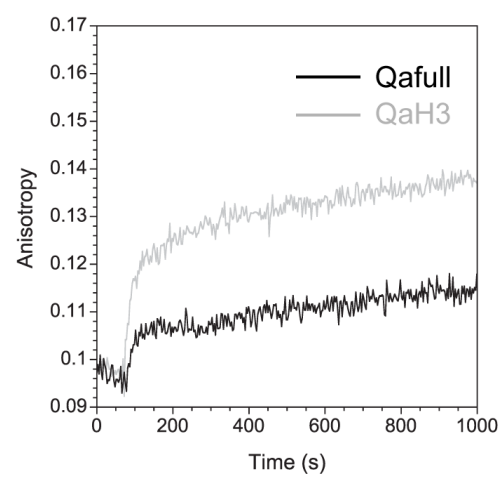

$Q^{*}+$ QabR PRE-MIXED

$(0.5 \mu \mathrm{M})$

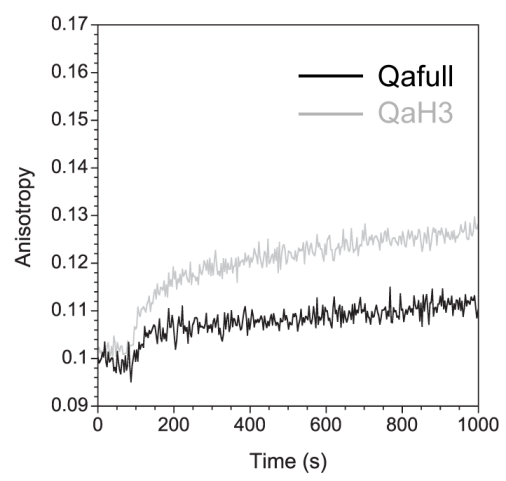

Figure 3.17: The transient QabR subcomplex exists in a dynamic equilibrium with its components

Different amounts of Qa-, Qb- and R-SNAREs were pre-incubated before mixing them with the $\sim 50 \mathrm{nM}$ fluorescent $\mathrm{Q}^{*}$-SNARE (Bet1p C85-OG). Either QaH3 or Qafull were included in the assembly reactions. Each measurement was started in the presence of sole $\mathrm{Qc}^{*}$ and the additional components were added after. To obtain the plot on the left side, the pre-incubated proteins were mixed such that their final concentrations in the cuvette were $\sim 2.7 \mu \mathrm{M}$. Similarly, for the plots in the middle and on the right, the final concentration of QabR mixes were $\sim 1.5 \mu \mathrm{M}$ and $\sim 0.5 \mu \mathrm{M}$, respectively. All experiments were performed at room temperature, in a total reaction volume of $1300 \mu \mathrm{l}$ and in a buffer containing $20 \mathrm{mM} \mathrm{NaH} \mathrm{PO}_{4} / \mathrm{Na}_{2} \mathrm{HPO}_{4} \mathrm{pH}$ : 7.4 and 200 $\mathrm{mM} \mathrm{NaCl}$

\subsection{Monitoring the effect of Sly1p on assembly of the ER-Golgi SNARES}

\subsubsection{Sly1p promotes SNARE assembly through the transient QabR subcomplex}

The experiments shown in the previous sections gave important insights into the dynamics and assembly of the ER-Golgi SNAREs. Having this groundwork in hand, I next aimed to investigate the role of Sly1p in the SNARE assembly machinery. I firstly wanted to observe whether Sly1p had any effect on the speed of SNARE complex formation. For this, the fluorescent $\mathrm{Qb}^{*}, \mathrm{Qc}^{*}$ and $\mathrm{R}^{*}$-SNAREs used before were mixed with their unlabeled partner SNAREs $(\approx 7 \mu \mathrm{M})$, either in the presence or in the absence of Sly1p $(\approx 11 \mu \mathrm{M})$. As in the previous experiments, the increase in fluorescence anisotropy was used to follow the process of SNARE complex formation (Figure 3.18). It should be noted that SNARE assembly reactions were first performed without pre-mixing of the ERGolgi SNAREs. In addition, in all experiments, full-length Sed5p (Qafull) was used, since Sly1p showed no interaction with the sole SNARE motif of Sed5p (QaH3) (Table 3.1). When the SNARE assembly reactions were followed in this way, SNAREs assembled faster in the presence of Sly1p, regardless of which SNARE was labeled to monitor the 

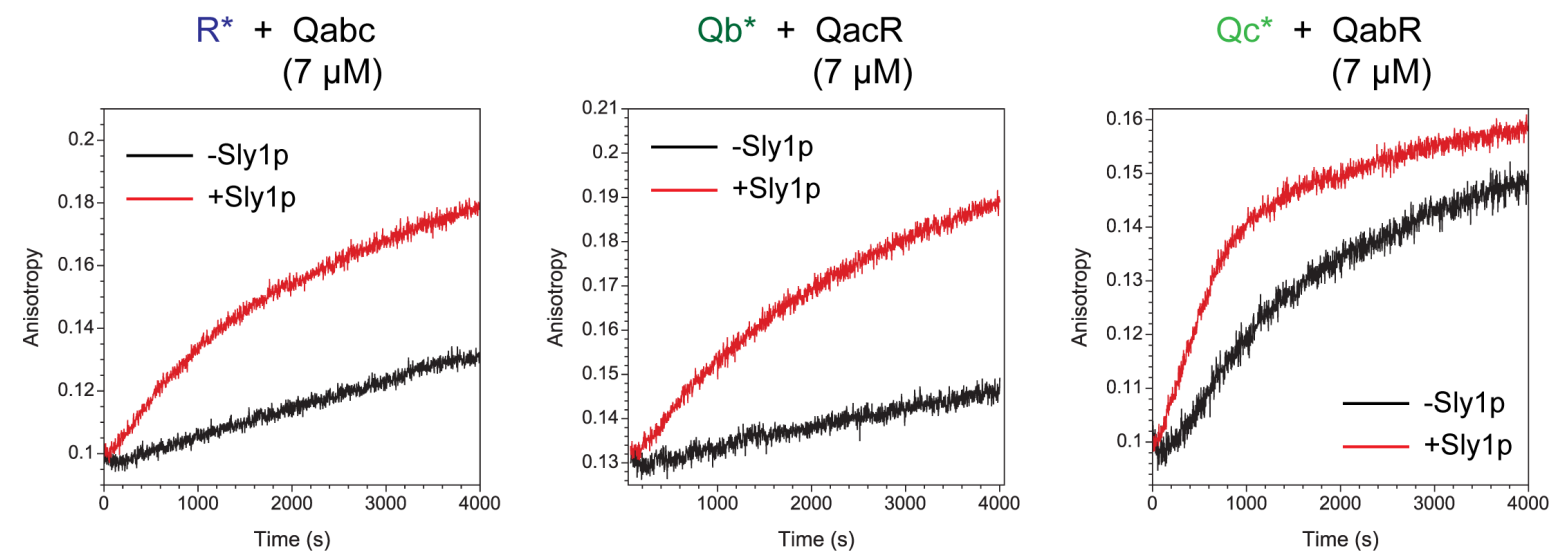

Figure 3.18: Assembly kinetics of ER-Golgi SNAREs in the presence of Sly1p

SNARE assembly was monitored by the increase in the anisotropy of $\sim 400 \mathrm{nM}$ fluorescent $\mathrm{Qb}^{*}$ - (Bos1p C181OG), Qc*- (Bet1p C85-OG) or R*-SNAREs (Sec22p C131-OG) upon mixing them with $7 \mu \mathrm{M}$ of their partners. In all experiments, the full-length Sed5p (Qafull) was used. The assembly mixes either included Sly1p (+Sly1p, red curves) or not (-Sly1p, black curves). $\sim 11 \mu \mathrm{M}$ of Sly1p was included in the reactions so that the ratio of Sly1p/ unlabeled SNAREs was approximately 1,5: 1. Each measurement was started immediately after mixing the components. Each experiment was performed in an ultra-micro fluorescence cell, in a total volume of 180 $\mu 1$.

SNARE complex formation. This revealed that Sly1p has a positive effect on the rate of SNARE assembly. It can be ruled out that the observed increase in fluorescence anisotropy is merely caused by the presence of the large protein Sly1p, since for each reaction the same endpoint in fluorescence anisotropy was reached in the absence or presence of Sly1p.

But how does Sly1p render the SNARE assembly reaction faster? In section 3.2.3, I showed that a transient intermediate formed between the Qa-, Qb- and R-SNAREs allows for much faster SNARE assembly compared to a reaction in which all SNAREs are mixed simultaneously. This raises the question whether Sly1p acts upon this transient QabR intermediate or is supporting another assembly pathway to enhance the kinetics of SNARE complex formation. In order to address this question, I added Sly1p to different SNARE premixes used in section 3.2.3. First, I pre-incubated Qafull, Qb- and Qc-SNAREs in the presence or absence of Sly1p, before mixing them with fluorescent $R^{*}$ in order to start the SNARE assembly reactions. Sly1p was added in excess in comparison to the unlabeled SNAREs in the premixes. As shown before in Figure $3.16 \mathrm{~A}$, the preassembled Qabc-SNAREs do not promote rapid SNARE assembly in the absence of Sly1p. Addition of Sly1p did not significantly accelerate SNARE complex formation (Figure 3.19 A). Thus, Sly1p apparently does not make use of a Qabc intermediate when enhancing the kinetics 
A

$A$

$\mathrm{R}^{*}+$ Qabc

$(7 \mu \mathrm{M})$

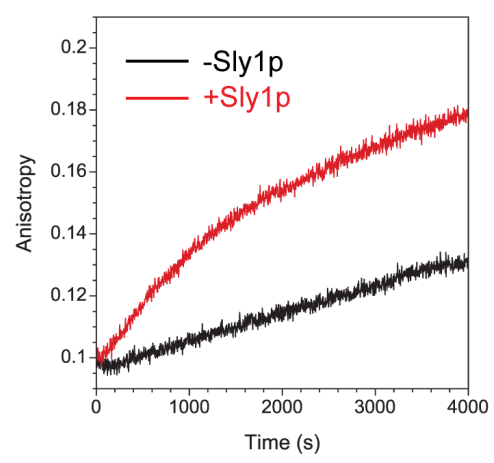

B

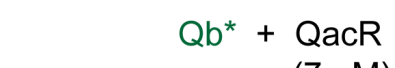

$(7 \mu \mathrm{M})$

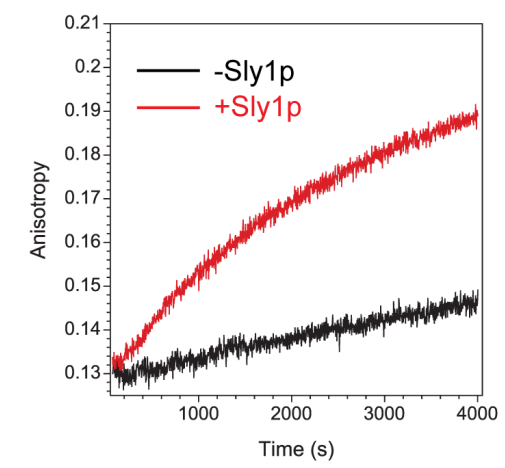

$\mathrm{R}^{*}+$ Qabc PRE-MIXED

$(7 \mu \mathrm{M})$

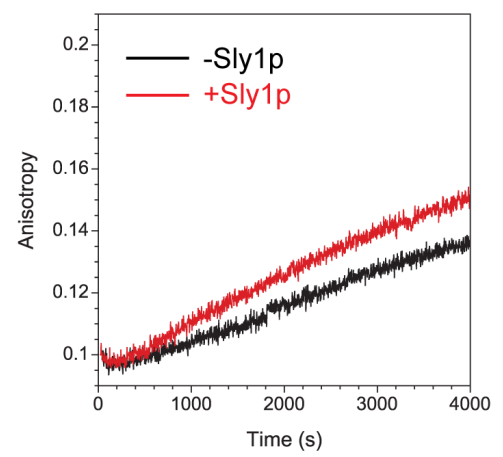

$\mathrm{Qb}^{*}+$ QacR PRE-MIXED

$(7 \mu \mathrm{M})$

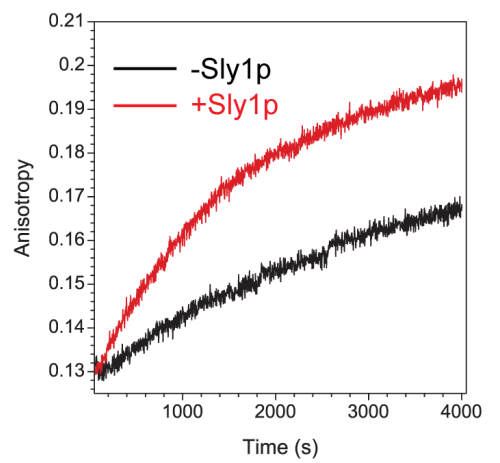

C

$\mathrm{QC}^{*}+\mathrm{QabR}$

$(2.7 \mu \mathrm{M})$

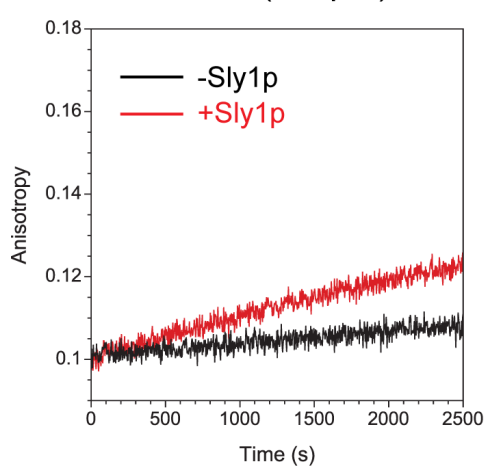

$\mathrm{Qc}^{*}+$ QabR PURIFIED

$(2.7 \mu \mathrm{M})$

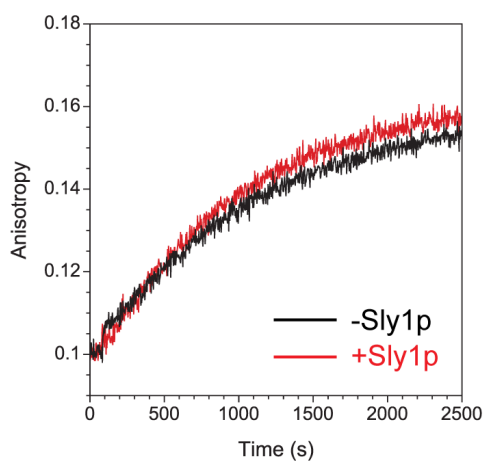

QC $^{*}+$ QabR PRE-MIXED

$(2.7 \mu \mathrm{M})$

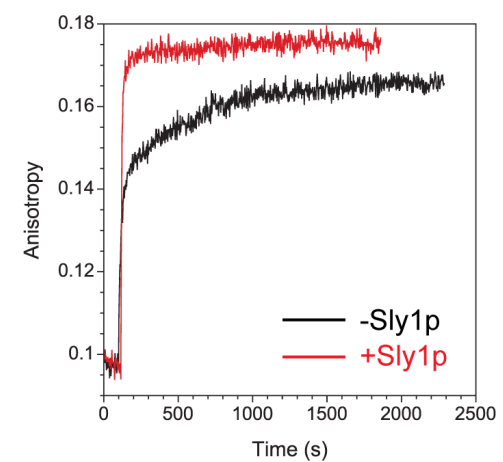

Figure 3.19: Assembly kinetics of ER-Golgi SNAREs, observed after pre-incubation of unlabeled SNAREs in the presence of Sly1p

A, B. SNARE assembly was monitored by the increase in the anisotropy of $\sim 400 \mathrm{nM}$ fluorescent $\mathrm{Qb}^{*}$ - (Bos1p C181-OG) or R*-SNAREs (Sec22p C131-OG) upon mixing them with their partner SNAREs. In all experiments, the full-length Sed5p (Qafull) was used. The plots on the left show the reactions where $\sim 7 \mu \mathrm{M}$ of unlabeled SNAREs were immediately mixed with the $\sim 400 \mathrm{nM}$ labeled SNARE (black curves). $\sim 11 \mu \mathrm{M}$ Sly1p was included in the reactions, shown in the red curves. On the other hand, the unlabeled SNAREs were preincubated overnight and added at $\sim 7 \mu \mathrm{M}$ in the cuvettes to obtain the plots on the right (black curves). When Sly1p was included in the premixes, the ratio of Sly1p/ unlabelled SNAREs was 1.5:1 (red curves). All experiments were performed at room temperature, in a total reaction volume of $180 \mu l$. 
C. Similarly, SNARE assembly was monitored by the increase in the anisotropy of $\sim 50 \mathrm{nM}$ fluorescent $\mathrm{Qc}^{*}$ SNARE (Bet1p C85-OG) upon mixing it with its partners. To obtain the plot on the left, $\sim 2.7 \mu \mathrm{M}$ of Qafull, Qb and R-SNAREs were sequentially added to the assembly reactions either in the presence of $\sim 4.5 \mu \mathrm{M}$ Sly1p (red curve) or in the absence of Sly1p (black curve). For the plot in the middle, either a purified Qafull-Qb-R complex (up to $\sim 2.7 \mu \mathrm{M}$, black curve) or a purified Sly1p-Qafull-Qb-R complex (up to $\sim 2.7 \mu \mathrm{M}$, red curve) was added on Qc*. To obtain the plot on the right, Qafull, Qb and R-SNAREs were pre-incubated either in the presence (red curve) or in the absence of Sly1p (black curve), before starting the reactions. The premix solutions contained $\sim 11 \mu \mathrm{M}$ of QabR-SNAREs and $\sim 17 \mu \mathrm{M}$ of Sly1p. The SNAREs in the pre-mixes were added at $\sim 2.7 \mu \mathrm{M}$ and Sly1p was added at $\sim 4.2 \mu \mathrm{M}$ in the assembly reactions. All experiments were performed at room temperature, in a total reaction volume of $1300 \mu l$.

of SNARE assembly. Comparable results were obtained when Sly1p was included in a premix of Qafull, Qc- and R-SNAREs (Figure 3.19 B).

Remarkably, when Sly1p was premixed with Qafull, Qb- and R-SNAREs and when the premixes were added in the reactions, SNARE complex formation was completed within seconds (Figure 3.19 C). It appears that Sly1p stabilized the transient QabR intermediate since the second, slower phase of the reaction, that was still clearly visible in the absence of Sly1p, almost entirely disappeared. Pre-incubation of Sly1p with only Qa- and Qb- or Qa- and R-SNAREs did not provide rapid SNARE assembly. SNARE assembly was as slow as in reactions in which the Qa-, Qb- and R-SNAREs were not premixed (data not shown). For another experiment, Sly1p, Qafull, Qb- and R-SNAREs were assembled into a complex and purified chromatographically. The Sly1p/QabR complex assembled with $\mathrm{Qc}^{*}$ in a similar speed compared to the QabR subcomplexes prepared without Sly1p (Figures $3.16 \mathrm{C}$ and $3.19 \mathrm{C}$ ). This indicates that Sly1p cannot transform a stable QabR subcomplex into a ready-available acceptor for the Qc SNARE. Together these findings support the notion that Sly1p accelerates SNARE assembly via supporting a transient intermediate formed between the Qa-, Qb- and R-SNAREs.

As explained in section 3.2.3, the transient QabR intermediate probably exists in a dynamic equilibrium with its constituents. If Sly1p indeed stabilized the QabR intermediate, Sly1p should shift the equilibrium reaction towards assembly of the QabR intermediate. In order to test this, similar to the experiments shown in figure 3.17 , the premixes were used at different concentrations in anisotropy measurements. The premixes, prepared in the presence or absence of Sly1p, were added to reactions at $\approx 2.7$ $\mu \mathrm{M}, \approx 1 \mu \mathrm{M}$, or $\approx 0.5 \mu \mathrm{M}$ (Figure $3.20 \mathrm{~A}$ ). In all cases, when Sly1p was included in the premixes, the rapid phase of the SNARE assembly reaction was more prominent, thus, probably more of the active QabR subcomplex was present. These results support the 
A

$\mathrm{QC}^{*}+$ QabR PRE-MIXED

$(2.7 \mu \mathrm{M})$

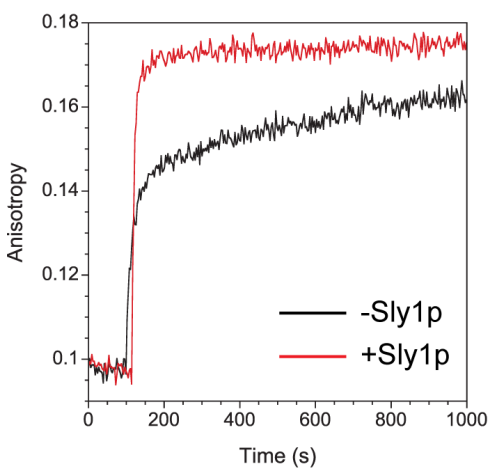

$\mathrm{QC}^{*}+$ QabR PRE-MIXED

$(1 \mu \mathrm{M})$

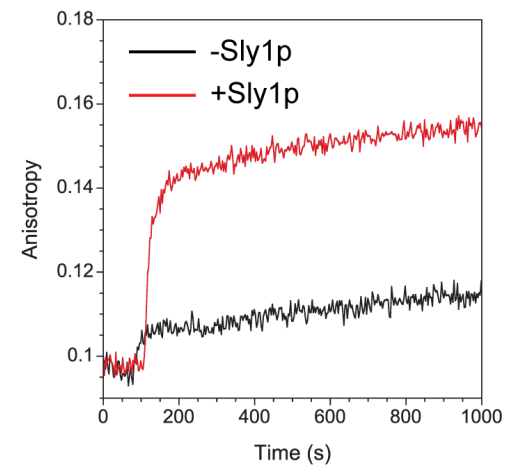

$\mathrm{QC}^{*}+$ QabR PRE-MIXED

$(0.5 \mu \mathrm{M})$

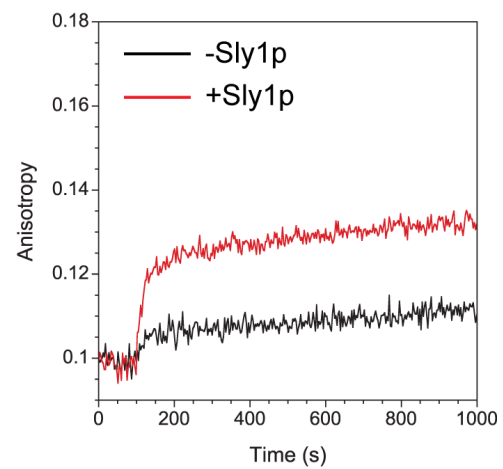

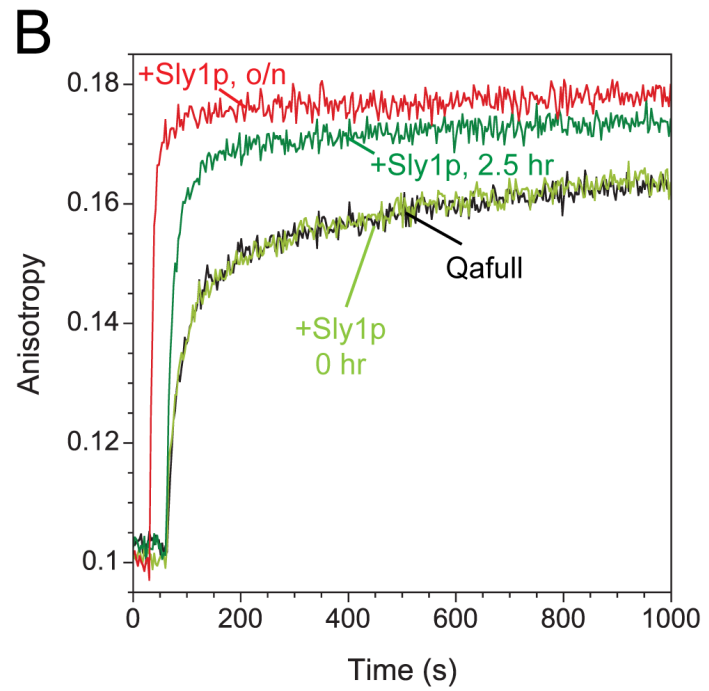

Figure 3.20: Equilibrium concentration of the QabR intermediate can be shifted in the presence of Sly1p

A. Different amounts of Qa-, Qb- and R-SNAREs were pre-incubated before mixing them with the $\sim 50 \mathrm{nM}$ fluorescent Qc*-SNARE (Bet1p C85-OG). The premixes were prepared with Qafull, either in the presence or in the absence of Sly1p. QabR-SNAREs were included at $\sim 11 \mu \mathrm{M}$ and Sly1p was included at $\sim 17 \mu \mathrm{M}$ to the premix solutions. The SNAREs in the premixes were added at $\sim 2.7 \mu \mathrm{M}$ or $\sim 1 \mu \mathrm{M}$ or $\sim 0.5 \mu \mathrm{M}$ in the reactions. B. The SNARE premixes were prepared in the presence or absence of Sly1p and were added at $\sim 2.7 \mu \mathrm{M}$ in the reactions. Different from A, Sly1p was included in the mixes of QabR-SNAREs after their preassembly (overnight). The assembly reactions were started either immediately after Sly1p addition (+Sly1p, $0 \mathrm{hr})$ or after incubation of Sly1p in the premixes for 2.5 hours (+Sly1p, $2.5 \mathrm{hr})$ or overnight $(+S l y 1 \mathrm{p}, \mathrm{o} / \mathrm{n})$.

notion that Sly1p indeed shifts the dynamic equilibrium towards the formation of a transient QabR intermediate. In another experiment, I added Sly1p to the QabR-SNAREs after they were allowed to preassemble for about 12 hours. Remarkably, no acceleration was observed, when the assembly reactions were started immediately after addition of Sly1p to the premixes. Interestingly, when Sly1p was added to the premix of the SNAREs 2.5 hours before the reaction was set off by adding labeled Bet1p, SNARE complex 

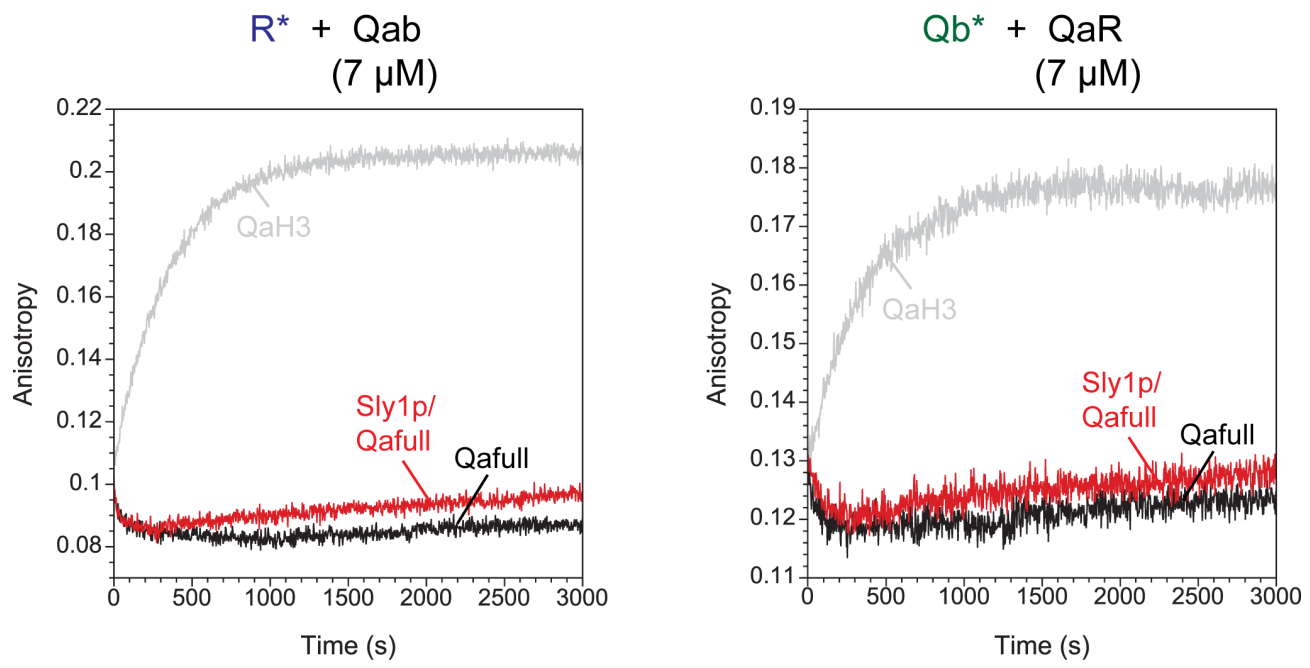

Figure 3.21: Sly1p does not excessively enhance the assembly kinetics of the QabR-intermediate

Assembly of the QabR-intermediate was monitored, either by mixing $\sim 400 \mathrm{nM}$ fluorescent $\mathrm{R}^{*}$ (Sec22p C131OG) with $\sim 7 \mu \mathrm{M}$ of Qa- and Qb-SNAREs (plot on the left); or by mixing $\sim 400 \mathrm{nM}$ fluorescent $\mathrm{Qb}^{*}$ (Sec22p C131-OG) with $\sim 7 \mu \mathrm{M}$ of Qa- and R-SNAREs (plot on the right). Either QaH3 (grey curves) or Qafull (black curves) were used, the latter also in the presence of $\sim 11 \mu \mathrm{M}$ of Sly1p (red curves). The unlabeled proteins were pre-incubated before the experiments. Each experiment was performed in an ultra-micro fluorescence cell, in a total volume of $180 \mu \mathrm{l}$. Similar anisotropy experiments were performed to follow the assembly kinetics of Qabc- and QacR-subcomplexes as well. These experiments are shown in the Appendix A.3.

formation was again clearly faster (Figure 3.20 B). Thus, Sly1p is capable of shifting the dynamic equilibrium towards the formation of a transient QabR intermediate.

Considering the results above, it seems likely that Sly1p increases the lifetime of a transient QabR intermediate. This might be achieved either by accelerating its formation or by slowing its dissociation. In order to test whether the QabR-SNAREs assemble faster in the presence of Sly1p, I again used fluorescence anisotropy. The formation of the QabR intermediate was monitored either by mixing Qa- and Qb-SNAREs with fluorescent $\mathrm{R}^{*}$, or by mixing Qa- and R-SNAREs with fluorescent $\mathrm{Qb}^{*}$ (Figure 3.21). When only the SNARE motif of Sed5p (QaH3) was used in the experiments, an increase in fluorescence anisotropy was observed. However, no significant change in anisotropy was monitored within the time course of the experiments (about an hour), when, instead the entire cytosolic portion of Sed5p (Qafull) was added, regardless whether the experiment was carried out in the absence or presence of Sly1p. This suggests that Sly1p at least does not appear to drastically accelerate the formation of the QabR intermediate. 


\subsubsection{Assembly and regulation of ER-Golgi SNAREs, followed by FRET experiments}

The fluorescence anisotropy experiments shown in the sections above provided valuable information on dynamics and regulation of the ER-Golgi SNAREs. However, the anisotropy approach has also some drawbacks. An anisotropy experiment, in essence, monitors only the rotational freedom of a fluorophore, hence it does not provide unambiguous information on what protein complex is formed. In order to support the results obtained by anisotropy experiments, I decided to follow the SNARE assembly via FRET (Förster Resonance Eneregy Transfer). FRET is a nonradiative energy transfer between two fluorophores, which are denoted as donor and acceptor. As a result, the intensity of the donor emission decreases, whereas that of the acceptor increases. To provide FRET, the fluorescence emission spectrum of the donor dye-molecule must overlap with the excitation spectrum of the acceptor dye-molecule. In addition, the dyemolecules must be in close proximity (typically 10-100 $\AA$ ), as FRET efficiency is proportional to the inverse sixth power of the distance between the fluorophores. SNARE complex formation can be monitored by FRET when a pair of fluorescent SNAREs is mixed with their unlabeled partner SNAREs.

For the FRET experiments I used the fluorescent SNAREs that were used in the anisotropy experiments. In each experiment, two fluorescent SNAREs, labeled as a FRET pair (each used at $\approx 50 \mathrm{nM}$ concentration), were mixed and incubated with an excess of their respective partner SNAREs. Upon testing several different combinations of labeled SNAREs, an Oregon Green-labeled Bet1p (Bet1p 1-118 C85-OG, denoted as QcOG) and a Texas Red-labeled Sec22p (Sec22p 126-186 C131-TR, denoted as $\mathrm{R}^{\mathrm{TR}}$ ) were giving a very good FRET signal and used for further experiments.

To monitor the assembly of the ER-Golgi SNAREs using FRET, QcOG and $\mathrm{R}^{\mathrm{TR}}$ were mixed with $\approx 5 \mu \mathrm{M}$ of Qa- and Qb-SNAREs. The donor and the acceptor fluorescence were simultaneously monitored (Figure $3.22 \mathrm{~A}$ ). When the entire cytosolic portion of Sed5p (Qafull) was used, the rate of fluorescence change was slow, in accord with the results obtained by fluorescence anisotropy. It should be noted that, no FRET was observed upon addition of only the Qafull or only the Qb-SNARE (data not shown). Therefore, the signal change observed very probably only denotes formation of the four-helix bundle SNARE complex, but not the formation of a subcomplex. Addition of Sly1p with the Qafull and 
$\mathrm{A} \quad \mathrm{Qc}^{\mathrm{OG}}+\mathrm{R}^{\mathrm{TR}}+\mathrm{Qab}$

$(5 \mu \mathrm{M})$

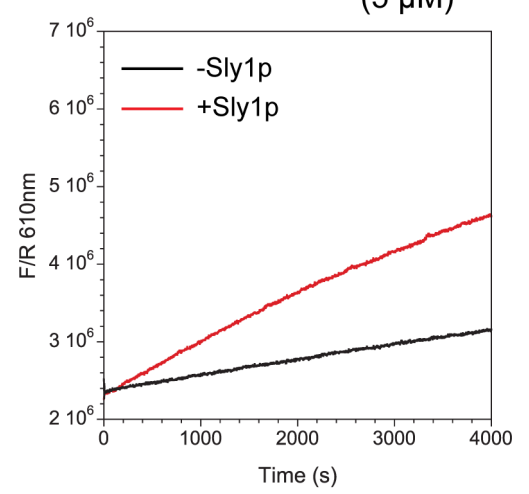

C R $^{\mathrm{TR}}+\mathrm{QabQC}^{\mathrm{OG}}$ PRE-MIXED

$(5 \mu \mathrm{M})$

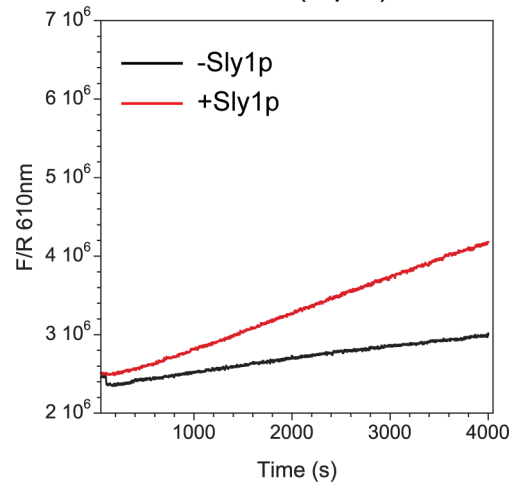

B $\mathrm{QC}^{\mathrm{OG}}+\mathrm{QabR}^{\mathrm{TR}}$ PRE-MIXED

$(5 \mu \mathrm{M})$
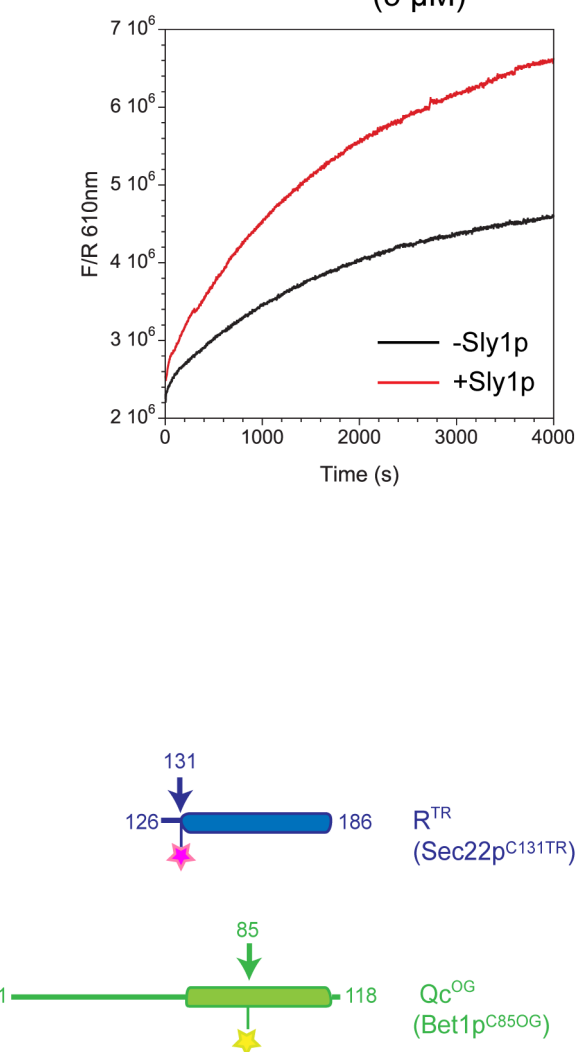

Figure 3.22: Assembly and regulation of ER-Golgi SNAREs, followed by FRET assays

A. $\sim 50 \mathrm{nM}$ of QcOG (Bet1p C85-OG) and $\sim 50 \mathrm{nM}$ of RTR (Sec22p C131-TR) were mixed with $\sim 5 \mu \mathrm{M}$ of Qafull and the Qb-SNARE in the presence or absence of $\sim 7 \mu \mathrm{M}$ Sly1p. After excitation of the donor molecule (QcOG) at $488 \mathrm{~nm}$, fluorescence emission from both the donor and the acceptor $\left(\mathrm{R}^{\mathrm{TR}}\right)$ molecules was monitored over time, at $520 \mathrm{~nm}$ and $610 \mathrm{~nm}$ respectively. For simplicity, only the corrected fluorescence signal from the acceptor molecule is shown. B, C. Different from A, QabR-SNAREs (shown in B) or Qabc-SNAREs (shown in C) were incubated overnight and added at $\sim 5 \mu \mathrm{M}$ in order to start the assembly reactions. The premix solutions were prepared in the presence of Sly1p as well (+Sly1p, shown in red curves). The ratio of Sly1p/ unlabeled SNAREs was around 1.5:1 in the premixes. Concentration of the fluorescent SNAREs was $\sim 50 \mathrm{nM}$ in all reactions. Schematic drawing of the fluorescent SNAREs used in the assays are shown next to the plots.

Qb-SNAREs accelerated the rate of fluorescence change (Figure $3.22 \mathrm{~A}$ ). Instead, when individual Sly1p or Sly1p-Qafull or Sly1p-Qb was used in the experiments, no FRET was observed. This indicates that Sly1p specifically accelerates formation of the four-helix SNARE bundle, but not a subcomplex. Thus, FRET experiments confirm the results obtained by fluorescence anisotropy.

In previous sections, I showed that a transient QabR intermediate is required for rapid SNARE assembly and Sly1p promotes and/ or stabilizes this intermediate. In order to 
confirm these results using a FRET approach, I again used the premixing strategy described above. For this, fluorescent $\mathrm{R}^{\mathrm{TR}}$ was preincubated with Qafull and Qb-SNARE, before adding fluorescent $\mathrm{QC}^{\mathrm{OG}}$ to start the FRET experiment. The rate of fluorescence change increased upon premixing, indicating faster SNARE assembly (Figure 3.22 B, black curve). In contrast, when fluorescent $\mathrm{QCOG}^{\mathrm{OG}}$ was preincubated with Qafull and Qb-SNARE, and then fluorescent $\mathrm{QC}^{\mathrm{OG}}$ was added, the kinetics of SNARE assembly was not enhanced (Figure 3.22 C, black curve). This supports the notion that SNARE assembly is faster upon preassembly of the QabR intermediate. Addition of Sly1p in either of the premix solutions accelerated the rate of SNARE complex formation (Figure 3.22 B, C, red curves). However, the acceleration was much more prominent upon addition of Sly1p to the QabR ${ }^{\mathrm{TR}}$ premix, again supporting the idea that Sly1p acts upon the QabR intermediate when rendering SNARE complex formation faster.

\subsubsection{Regulation of SNARE assembly by the mutants of Sed5p and Sly1p}

In the above sections, the biochemical interplay between Sly1p and the ER-Golgi SNAREs was studied using the entire cytosolic portions or SNARE domains without further modifications. Next, mutated versions of Sed5p and of Sly1p were used in the assays developed in this study in order to analyze the role of the two binding sites between the two proteins during SNARE complex formation.

Since the results shown in section 3.1.2 revealed that the N-peptide region of Sed5 is crucial for the formation of a high-affinity complex between Sed5 and Sly1, I first tested mutations in this region. As mentioned previously, the F10A mutant of Sed5p is considered to be unable to bind Sly1p, yet it does not affect the transport kinetics of protein cargo between ER and Golgi in vivo (Yamaguchi et al., 2002, Peng and Gallwitz, 2004). On the other hand, ITC experiments shown above (figure 3.8) demonstrate that the F10A mutant can still bind Sly1p, yet with very low affinity. In order to test the effect of Sly1p on SNARE assembly when N-peptide binding is weakened, I used the F10A mutant of Sed5p in the anisotropy assay. I preincubated the F10A mutant, Qb- and R-SNAREs in the presence or absence of Sly1p, before adding fluorescent Qc* (Figure 3.23 A). The rate of SNARE assembly in the absence of Sly1p was comparable to wild-type. By contrast, the rapid phase of the reaction was somewhat less prominent in the presence of Sly1p. 

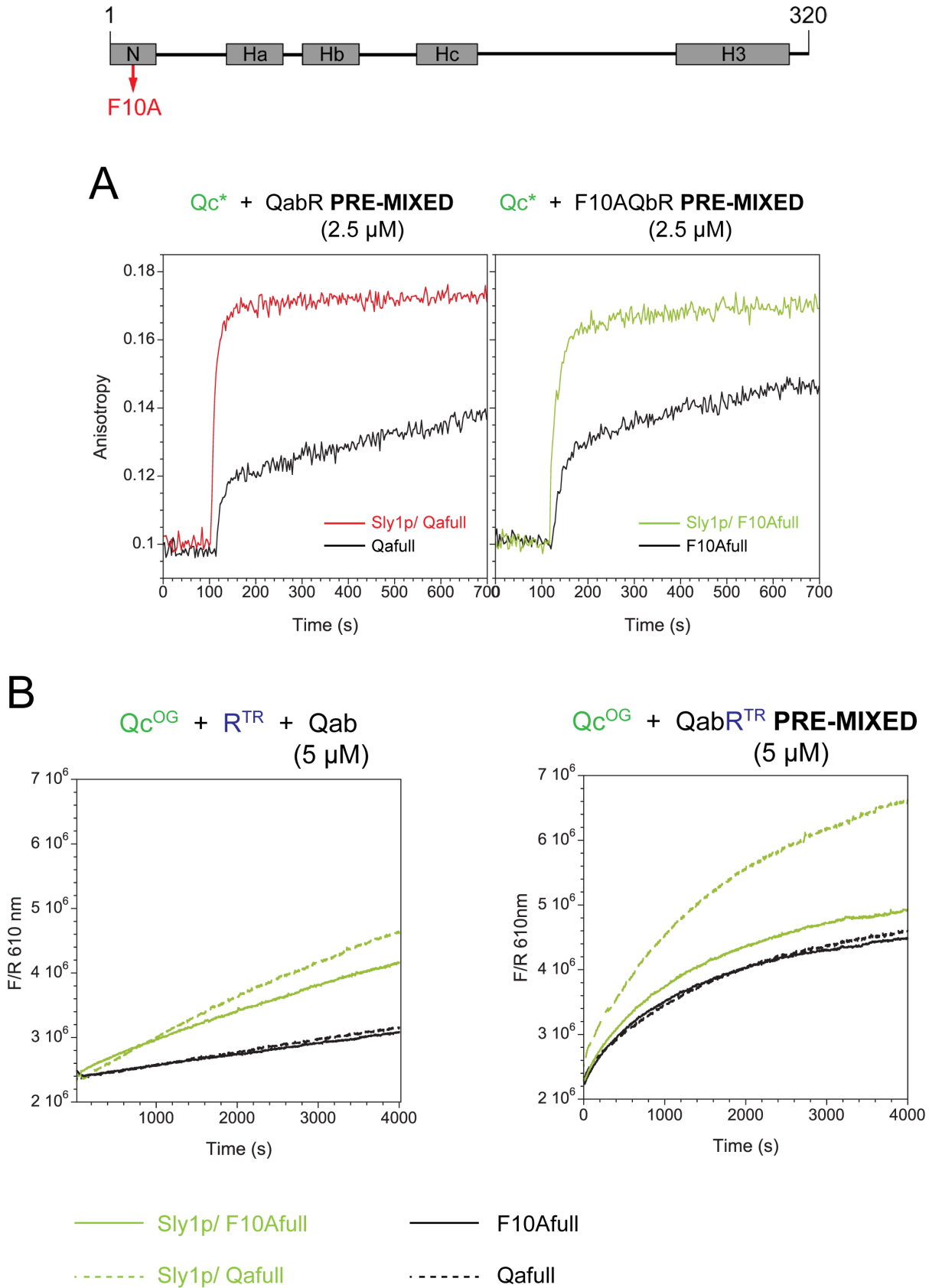

Figure 3.23: SNARE assembly kinetics followed by fluorescence anisotropy and FRET, in the presence of the F10A mutant of Sed5p

A. Bos1p (Qb), Sec22p (R) and the F10A mutant of Sed5p (Qafull) were preincubated in the presence or absence of Sly1p, before mixing them with the fluorescent Qc* (Bet1p C85-OG). Concentration of SNAREs in the premix solutions was at $\sim 11 \mu \mathrm{M}$ and that of Sly1p was at $\sim 17 \mu \mathrm{M}$. The SNAREs in the premixes were added at $\sim 2.5 \mu \mathrm{M}$ in the reactions, and accordingly Sly1p, at $\sim 3.7 \mu \mathrm{M}$. The same experiments were performed with the wild-type Sed5p to obtain the plot on the left. B. FRET assays were performed as described in figure 3.22, either by no premixing of the components or by premixing the QabRTR-SNAREs. Either wild-type Sed5p (Qafull) or the F10A mutant (F10Afull) were used in the reactions. Schematic drawing of the F10Afull is shown on top of the figure. 
21
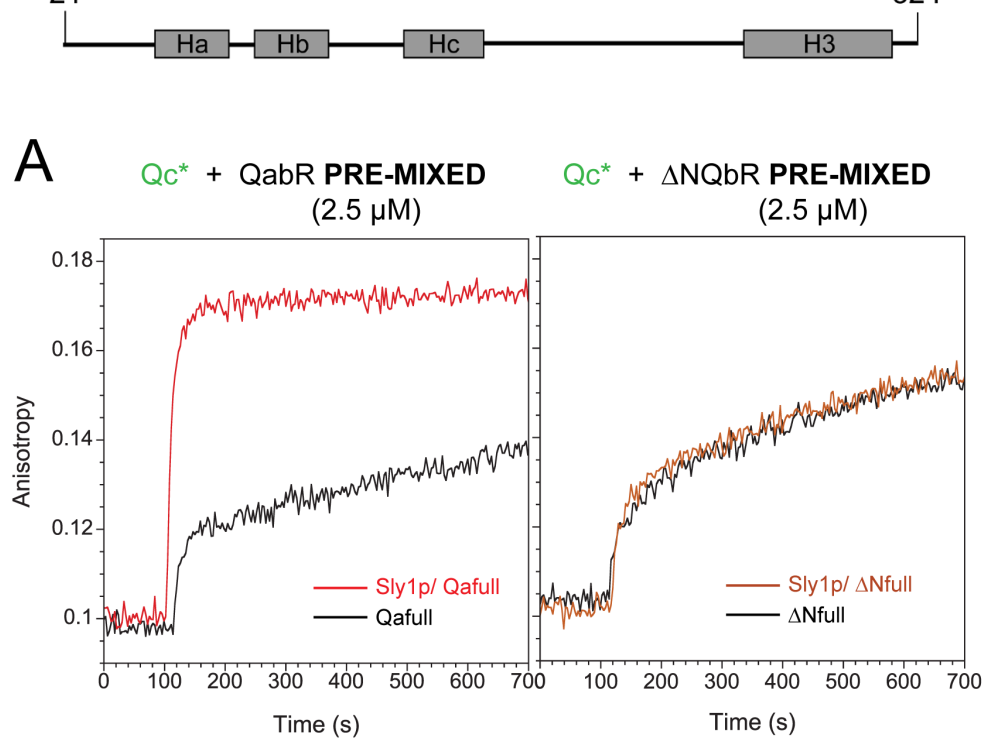

B

$\mathrm{Qc}^{\mathrm{OG}}+\mathrm{R}^{\mathrm{TR}}+\mathrm{Qab}$

$(3 \mu \mathrm{M})$

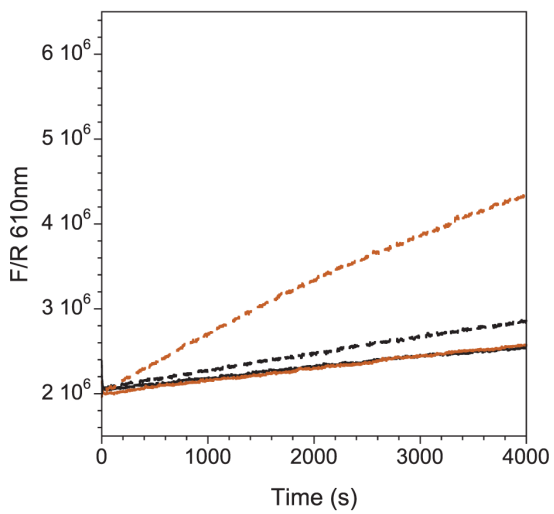

Sly1p/ $\Delta$ Nfull

Sly1p/ Qafull
$\mathrm{Qc}^{\mathrm{OG}}+\mathrm{QabR}^{\mathrm{TR}}$ PRE-MIXED $(3 \mu \mathrm{M})$

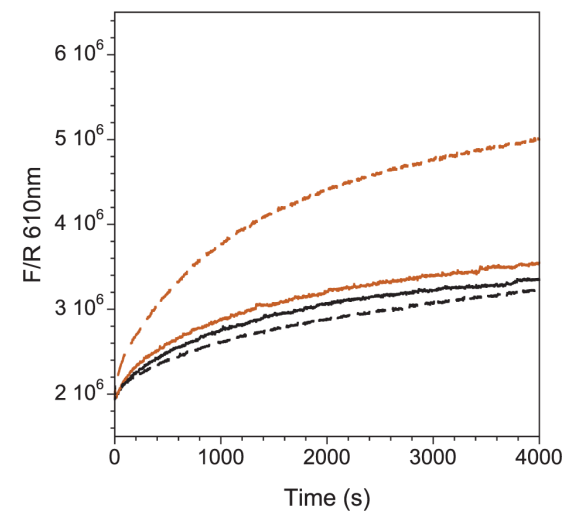

$\Delta$ Nfull

Qafull

Figure 3.24: SNARE assembly kinetics followed by fluorescence anisotropy and FRET, in the presence of the $\Delta \mathrm{N}$-mutant of Sed5p

A. Bos1p (Qb), Sec22p (R) and the $\Delta N$ mutant of Sed5p ( $\Delta$ Nfull, residues 21-324) were preincubated in the presence or absence of Sly1p, before mixing them with the fluorescent $\mathrm{Qc}^{*}$ (Bet1p C85-OG). The anisotropy assay was performed as described in figure $3.23 \mathrm{~A}$. The same experiments were performed with the wild-type Sed5p to obtain the plot on the left. B. FRET assays were performed as described in figure 3.22, either by no premixing of the components or by premixing the QabRTR-SNAREs. Either wild-type Sed5p (Qafull) or the $\Delta \mathrm{N}$ mutant $(\Delta \mathrm{Nfull})$ were used in the reactions. Schematic drawing of the $\Delta \mathrm{Nfull}$ is shown on top of the figure. 
I also tested the F10A mutant using the FRET assays. Using this approach, the accelerating effect of Sly1p on SNARE assembly was much less pronounced, regardless whether $\approx 5 \mu \mathrm{M}$ premixed QabRTR_SNAREs were used or no premixing was performed (figure $3.23 \mathrm{~B}$ ). This indicates that the F10A mutant of Sed5p is an inferior substrate for Sly1p as compared to wild-type Sed5p. There are probably two reasons that the loss of the accelerating role of Sly1p was more clear when using the FRET approach instead of the anisotropy approach. Firstly, the FRET approach is much more specific as it only detects the assembly of the four-helix bundle SNARE complex, whereas the fluorescence anisotropy can also increase upon several subcomplexes. Secondly and probably more importantly, due to overall lower SNARE protein concentrations - note that for the FRET approach the two labeled SNARE proteins, Sec22p and Bet1p, were used at low concentration $(50 \mathrm{nM})$, whereas for the fluorescence anisotropy approach only Bet1 was used at $50 \mathrm{nM}$ - the likelihood for the formation of the transient QabR acceptor complex was less pronounced during the FRET experiments. Note that due to the reduced amount of transient QabR acceptor complex formed during the FRET experiments, the rate of SNARE complex formation appears to be slower compared to the anisotropy approach. With such a reduced tendency for the formation of the QabR acceptor complex, the FRET approach was apparently better suited to reveal that the reduced affinity of Sly1p for the F10A mutant (according to the ITC experiments shown in section 3.1.2, the $\mathrm{K}_{\mathrm{d}}$ of their interaction is only $\approx 200 \mathrm{nM})$ compared to wild-type Sed5p $\left(\mathrm{K}_{\mathrm{d}}<1 \mathrm{nM}\right)$ has a strong impact on the SNARE complex-promoting activity of Sly1p. Still, I noted that Sly1p was able to somewhat accelerate the formation of the SNARE complex when the F10A mutant was used, suggesting that the low affinity between Sly1p and the F10A mutant was still sufficient for Sly1p to act upon the SNARE machinery.

In order to test whether reducing the affinity between Sly1 and Sed5 even further, I next tested the Sed5p mutant lacking the N-peptide (residues 21-324, denoted as $\Delta \mathrm{N}-\mathrm{Sed} 5 \mathrm{p}$ ). As shown in section 3.1.2 (see Figure 3.5), no interaction was detected between $\Delta$ N-Sed5p and Sly1p using ITC. As the F10A mutant, $\Delta \mathrm{N}-$ Sed5p formed SNARE complexes at a similar speed as wild-type Sed5p. However, the effect of Sly1p on SNARE assembly was completely abolished in the presence of $\Delta \mathrm{N}-\mathrm{Sed} 5 \mathrm{p}$, no matter whether the anisotropy or FRET approach was used (Figure 3.24). Therefore, in the absence of N-peptide of Sed5p, Sly1p does not accelerate SNARE complex formation. 

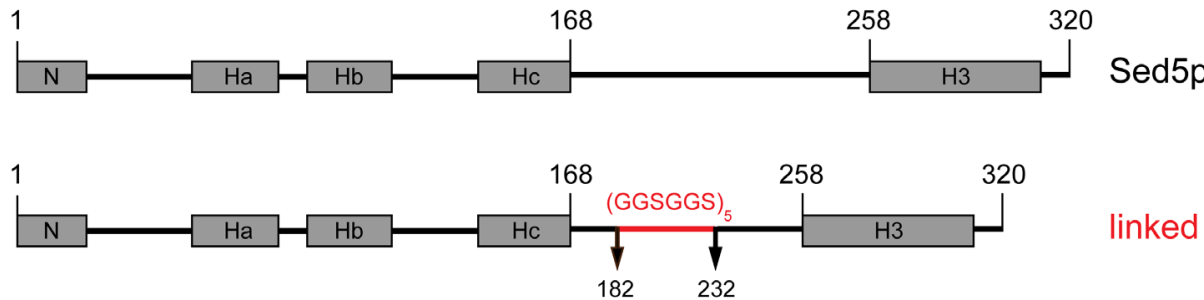

$Q_{C}{ }^{O G}+Q^{2}{ }^{T R}$ PRE-MIXED

$(3 \mu \mathrm{M})$

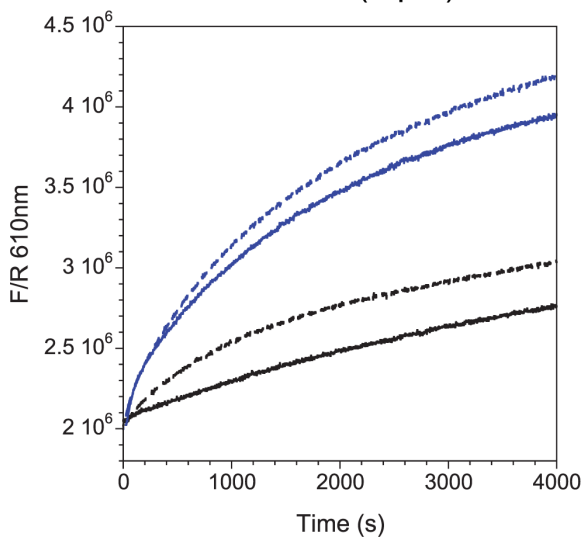

Sly1p/linked

..... Sly1p/ Qafull

linked

Qafull

Figure 3.25: SNARE assembly kinetics in the presence of a Sed5p variant containing a modified linker

In order to replace the entire linker region of Sed5p with a flexible protein fragment, residues 182-232 were substituted with 5 GGSGGS repeats (see section 2.1.5). The resulting Sed5p variant is named as "Sed5plinked" and its schematic drawing is shown on top of the figure. FRET assay was performed as described in figure 3.22, by premixing the linked with Qb- and RTR-SNAREs. SNARE assembly kinetics was compared to wild-type Sed5p (Qafull), in the presence and in the absence of Sly1p.

The linker region between the Habc domain and the SNARE motif is thought to play substantial role in stabilizing the closed conformations of Syntaxin 1a and Sso1p (Munson et al., 2000, Misura et al., 2000). In order to test the effect of the linker region of Sed5p on SNARE complex formation, I wanted to design mutations of the linker region. Unfortunately, as the crystal structure of the Sly1p/Sed5p complex only contains the Npeptide region of Sed5p, there is no structural information for the Sed5p linker available. In addition, the sequence in this region is poorly conserved (see Appendix A.1). I eventually replaced the entire linker region with a flexible linker, formed of five GGSGGS repeats (Figure 3.25). A similar peptide linker had been used successfully in a previous study (van Dongen et al., 2007). For simplicity, the mutant protein was named as "Sed5plinked". Purification of Sed5p-linked was challenging, since the protein tended to form oligomers. Nevertheless, eventually I was able to probe Sed5p-linked's ability to form SNARE complexes using the FRET assay described above (Figure 3.22). In Figure 3.25, it is 
shown that SNARE complex formation was somewhat slower when Sed5p-linked was used instead of wild-type Sed5p. Interestingly however, Sly1p was still able to accelerate SNARE complex formation when Sed5-linked was used instead of wild-type Sed5p, suggesting that Sly1p does not require the linker region of Sed5p to act upon SNARE assembly. 


\section{Chapter 4}

\section{Discussion}

So far, two molecular machineries have been identified to be essential for canonical vesicle fusion in the secretory pathway: SM proteins and SNAREs. The core engine of membrane fusion is thought to be the so-called SNARE complex, a four-helical bundle formed by SNARE proteins residing on opposite membranes. The free energy released upon formation of the SNARE complex, according to this idea, is used to overcome the energy barrier required for fusion (Hanson et al., 1997, Lin and Scheller, 1997). SM proteins are thought to be the indispensible regulatory elements of this core fusion machinery. In general, the high-affinity interaction partners of SM proteins are Qa-SNAREs (syntaxins). As two different binding modes were discovered a decade ago, it was proposed that SM proteins can bind to their cognate syntaxins in two different binding modes (reviewed in Toonen and Verhage, 2003). In mode 1, a closed conformation formed by the syntaxin is grasped and stabilized in the inner cavity of the SM protein exemplified by the structure of Munc18a/Syntaxin 1a, whereas mode 2 involves binding of a short N-peptide motif of the syntaxin on the outer surface of the SM protein exemplified by the structure of Sly1p/Sed5p (see Figure 1.4). Importantly, these two binding modes may not be exclusively used, since recent examples demonstrate that the SM protein can use both binding sites simultaneously to bind the syntaxin (Burkhardt et al., 2008, Furgason et al., 2009, Peng et al., 2010, D'Andrea-Merrins et al., 2007, Johnson et al., 2009, Burkhardt et al., 2011). Inferred from Munc18a/Syntaxin 1a interaction, the binding status of the SM protein to the two spatially distinct sites might affect the conformation of the SM protein/syntaxin pair (Burkhardt et al., 2008). Therefore, SM proteins might have an innate mechanism to control the structural configuration of the syntaxin, thereby orchestrating the SNARE assembly process. SM proteins have also been suggested to 
remain associated with the assembled SNARE complexes and to promote fusion by acting on them (Carr and Rizo, 2010, Sudhof and Rothman, 2009, Burkhardt et al., 2008).

The molecular mechanism of the functional interaction between SM proteins and SNAREs is largely controversial. One of the underlying reasons could be that the specific action of an SM protein at each of the sequential steps of a SNARE assembly process has not been dissected. A closed conformation adopted by syntaxins could be a built-in "off" switch for autoregulation of SNARE assembly, since this conformation is thought to render the SNARE motif of the syntaxin inaccessible for its partner SNAREs (Munson et al., 2000, Misura et al., 2000). Therefore, one of the major functions of an SM protein could be to modulate this "off" switch and thus, the availability of the syntaxin SNARE motif. The modulation might assure that productive trans-SNARE pairing occurs at the correct location and time. The modulation might also involve some sort of a "proofreading" activity so that that the syntaxin is "solely" accessible for its partner SNAREs. Furthermore, SM protein might insure that the syntaxin uses a particular assembly pathway for formation of the SNARE complex. In other words, the SM protein might support distinct folding intermediates, but exclude others along the way for SNARE complex formation. In order to recapitulate the interplay between SM proteins and SNAREs in vitro, the models described above must be tested. After this step, the next goal can be addition of accessory factors, so that the sequential regulatory steps can be faithfully reproduced using in vitro tools. Possibly, for one or more of the regulatory steps in the course of SNARE assembly, the SM protein might work in conjunction with other factors such as tethering/ docking or priming factors in vivo. Another future goal can be to investigate a putative role of the SM protein on the assembled SNARE complex.

The aim of this study was to examine the specific action of the SM protein Sly1p on the assembly of the ER-to-Golgi SNAREs Sed5p (Qa), Bos1p (Qb), Bet1p (Qc) and Sec22p (R). Studies in yeast indicate that Sly1p is essentially required for the ER-to-Golgi transport (Ossig et al., 1991). The high-affinity binding partner of Sly1p is the syntaxin Sed5p (Grabowski and Gallwitz, 1997, Kosodo et al., 1998, Yamaguchi et al., 2002, Bracher and Weissenhorn, 2002). Until now, Sly1p/Sed5p interaction was thought to involve solely the $\mathrm{N}$-peptide motif of Sed5p, although former studies could not rule out a weak/transient binding of the remainder of Sed5p as well (Bracher and Weissenhorn, 2002). It was also not known whether Sed5p can adopt a closed conformation as a single molecule or when bound to Sly1p. Action of Sly1p in the course of the SNARE complex formation was also 
largely unknown. Nonetheless, according to earlier studies, Sly1p has a proofreading function such that Sly1p-bound Sed5p does not form nonphysiological SNARE complexes in vitro (Peng and Gallwitz, 2002). The assembly mechanism of the ER-Golgi SNAREs and the dynamics of their interactions have not been studied, and therefore, regulation of Sly1p at distinct steps in the assembly pathway was unknown up to now. For understanding the interplay between Sly1p and the ER-Golgi SNAREs, I attempted to solve the problems described above.

\subsection{Biochemical characterization of individual Sed5p and the Sly1p/Sed5p complex}

As many other syntaxins, yeast Sed5p has an N-terminal regulatory domain composed of a short N-peptide motif (residues 1-21), the Habc domain (53-168) and a linker region (168-258) (Yamaguchi et al., 2002). Previously, solution structure of the N-terminal domain was studied by NMR, identifying the autonomously folded Habc domain (Yamaguchi et al., 2002). However, conformation of the entire cytosolic portion of Sed5p has not been investigated. I initially aimed to find out whether individual Sed5p can adopt a closed conformation, as a closed conformation of Sed5p would affect its accessibility for SNARE complex formation. In addition, if already individual Sed5p could form a closed conformation, so the reasoning, this configuration can bind to Sly1p and might be rearranged by Sly1p during SNARE assembly.

Several lines of evidence obtained in this study indicate that individual Sed5p can indeed adopt a closed conformation. First, using CD spectroscopy I measured the thermal stabilities of the two Sed5p fragments, Sed5p (1-210), comprising the N-peptide, the Habc domain and a stretch of the linker region, and Sed5p (1-320), comprising the entire cytosolic portion of the syntaxin. Accordingly, the longer Sed5p fragment including the SNARE motif $\left(\mathrm{Tm} \approx 72^{\circ} \mathrm{C}\right)$ is significantly more stable than the shorter one $\left(\mathrm{Tm} \approx 60^{\circ} \mathrm{C}\right)$. Thus, the N-terminal portion of Sed5p seems to be stabilized in the presence of the SNARE motif. Second, Sed5p (1-210) containing the N-terminal domain of the syntaxin formed a complex with Sed5p (211-320) containing the SNARE motif, as monitored by both native gel electrophoresis and size-exclusion chromatography. This provides evidence for an intramolecular interaction within Sed5p. Third, the interaction between 
the two fragments was confirmed using ITC. Accordingly, Sed5p (1-210) and Sed5p (211320) fragments form a $1: 1$ complex with an unfavorable enthalpy $(\Delta \mathrm{H} \approx+6 \mathrm{kcal} / \mathrm{mole})$. The entropy-driven binding of two fragments indicates that non polar-regions of Sed5p could be buried upon formation of closed conformation.

On one hand, my findings demonstrate that Sed5p can adopt a closed conformation. On the other hand, it remains possible that Sed5p is preferably closed, but it can still switch between the open and closed states to some extent. The Habc domain binds the SNARE motif with a very low affinity as measured by ITC $\left(K_{d} \approx 2.7 \mu M\right)$. Nevertheless, this lowaffinity interaction might support the conformational re-arrangements that could occur during SNARE assembly. Further biophysical and/or structural characterization will be necessary to test these models. It needs to be mentioned that I attempted to crystallize a Sed5p fragment (residues 45-283), analogous to a Sso1p fragment that was crystallized before (Munson et al., 2000), but no crystals were obtained. Then, I performed limited proteolysis experiments with Sed5p to identify discernible, stable fragments, which could correspond to a closed conformation of Sed5p. However, no such fragments were found, indicating disordered and flexible regions within the syntaxin, which might hinder crystallization (data not shown). This is perhaps not extraordinarily surprising because previous NMR data shows that the flexible loop between the $\mathrm{Hb}$ and Hc helices of Sed5p is unusually long (Yamaguchi et al., 2002). Furthermore, the linker region between the Habc domain and the SNARE motif of Sed5p ( $\approx 75$ residues) is much longer than the linker regions of Sso1p and of Syntaxin 1a ( $\approx 30$ residues), the two other syntaxins, which were shown to form closed conformations. Thus, for structural characterization of Sed5p, other tools than X-ray crystallography, maybe NMR could be used.

After establishing that Sed5p can adopt a closed conformation, I investigated whether Sly1p can interact with the closed form of Sed5p. For this, the energetical parameters of Sly1p/Sed5p interaction were measured using ITC. First, I titrated Sly1p with the entire cytosolic portion of Sed5p (residues 1-320). Accordingly, the interaction is exothermic $(\Delta \mathrm{H}$ $\approx 24 \mathrm{kcal} / \mathrm{mole})$, occurs in a 1:1 stoichiometry and with a high affinity $\left(\mathrm{K}_{\mathrm{d}} \approx 0.25 \mathrm{nM}\right)$. To dissect the interacting regions of Sed5p with Sly1p, I generated several truncated forms of Sed5p. Two of the fragments were N-terminally truncated, Sed5p (21-324) lacking the Npeptide and Sed5p (211-320) lacking the complete N-terminal domain. Interestingly, none of the N-terminally truncated variants of Sed5p bound to Sly1p, indicating that the Npeptide binding is essential for Sly1p interaction. The C-terminally deleted fragments 
used in the study were Sed5p (1-21), containing only the N-peptide and Sed5p (1-210), containing also the Habc domain and a part of the linker. These fragments bound Sly1p with a comparable affinity to the entire cytosolic region $\left(\mathrm{K}_{\mathrm{d}} \approx 1.47 \mathrm{nM}\right.$ for Sed5p $1-21, \mathrm{~K}_{\mathrm{d}} \approx$ $0.24 \mathrm{nM}$ for Sed5p 1-210). It was notable, however, that the enthalpy change upon Sly1p binding was smaller when these fragments were used $(\Delta \mathrm{H} \approx-16 \mathrm{kcal} /$ mole for Sed5p 1-21, $\Delta \mathrm{H} \approx-18 \mathrm{kcal} /$ mole for Sed5p 1-210), indicating a loss in the interaction surface of each of the protein complexes. These findings strongly suggest that not only the N-peptide motif but also the remainder of Sed5p participates in Sly1p interaction. Nonetheless, the Npeptide motif seems to be sufficient for high-affinity binding and the remaining portion of Sed5p interacts much weaker.

Each of the C-terminally deleted Sed5p variants (residues 1-21, 1-210 and 1-320) bound Sly1p with affinities in the low nanomolar range. Because the titration curves of each fragment had steep transitions, it was not possible to accurately determine and compare the dissociation constants of these fragments using conventional, non-competitive ITC measurements. In order to compare the affinities of the Sed5p fragments using another approach, fluorescence anisotropy experiments were performed. Sed5p (1-21), Sed5p (1210) and Sed5p (1-320) were each labeled with fluorophores at residue 22, adjacent to the N-peptide. When binding of the labeled fragments to Sly1p was monitored using anisotropy, the on-rates of all Sed5p constructs were rapid and comparable. Next, dissociation of the labeled constructs was followed using a competitive dissociation approach. The off-rates of Sed5p (1-210) and Sed5p (1-320) fragments were significantly slower compared to Sed5p (1-21), indicating that these longer fragments were held much tighter by Sly1p. Thus, using anisotropy approach, I was able to resolve the difference between the affinities of the sole N-peptide and the longer Sed5p fragments. Thus, the anisotropy data supported the idea that not only the N-peptide but also the other regions of Sed5p contributes to Sly1p binding. Since the off-rates of Sed5p (1-210) and (1-320) were very similar, it was not clearly evident whether SNARE motif participates in the interaction. In order to dissect the binding studies specifically up to the SNARE motif, I labeled several positions on the SNARE motif of Sed5p to use in EPR experiments. EPR spectra were collected from each position, when Sed5p was either isolated or in complex with Sly1p. Some residues (e.g., C282, C302) were merely immobilized upon Sly1p interaction, but in general, no large structural rearrangements were observed, indicating that SNARE motif very weakly binds Sly1p. 
As summarized above, I dissected the interaction surface between Sly1p and Sed5p using several biophysical tools. Accordingly, N-peptide motif is the major contributor to Sly1p binding, but the Habc domain and the SNARE motif also weakly interact with Sly1p. But how could one be sure that it is the closed conformation of Sed5p which binds to Sly1p? Strong evidence for this comes from the experiments that demonstrated that individual Sed5p can adopt a closed conformation. Additional indirect evidence comes from an ITC experiment, in which Sly1p was titrated to a purified complex of Sed5p (1-210) and (211320) fragments. The heat change upon titration of the purified complex $(\Delta \mathrm{H} \approx-22.7$ $\mathrm{kcal} /$ mole) was almost indistinguishable from that of the entire cytosolic portion of Sed5p $(\Delta \mathrm{H} \approx-23.9 \mathrm{kcal} / \mathrm{mole})$. This suggests that the SNARE motif of Sed5p participates in Sly1 $\mathrm{p}$ binding as a separate entity, likely forming a closed conformation with the Habc domain. In order to support this idea more directly, it could be tested in the future whether a purified Sly1p/Sed5p (1-210) complex binds to Sed5p (211-320). Size exclusion chromatography or ITC could be used for the binding experiments. If the closed conformation of Sed5p binds Sly1p analogous to the Munc18a/Syntaxin 1a interaction, the residues critical for the Munc18a-closed syntaxin interaction could be important for Sly1p/Sed5p complex as well. For instance, the point mutation I233A within the SNARE domain of Syntaxin 1a strongly interferes with the binding to the central cavity of Munc18a (Wu et al., 2001, Burkhardt et al., 2008). The Isoleucine residue of Sed5p (I290) at the homologous position could be mutated into Alanine to test whether it affects Sly1p binding. If the point mutation I290A interferes with binding of the closed Sed5p, this would possibly be observed by ITC as a decrease in the binding enthalpy.

Several lines of evidence provided in this study indicate that the second binding site of Sly1p/Sed5p complex could be occupied by the closed conformation of Sed5p. This supports the notion that all SM proteins can bind their cognate syntaxins using two spatially separated interaction sites, the N-peptide and the closed conformation. Similar quantitative binding studies might be needed to possibly expose a second binding site for other SM proteins that have previously been suggested to bind their syntaxin's N-peptide only. Nevertheless, only a few such studies have been carried out so far. In one example, preliminary ITC experiments that have been performed for the interaction of vertebrate Vps45 and Syntaxin 16 revealed the presence of a second binding site (Burkhardt et al., 2008). Similar to Sly1p/Sed5p interaction, the N-peptide motif of Syntaxin 16 is already sufficient for high-affinity binding, but the study showed that the remainder of Syntaxin 
16 also interacts with Vps45. A second binding site between Vps45p and Tlg2p, i.e. the yeast homologs of Vps45 and Syntaxin 16, has also been recently characterized, but in this case the two binding sites seem to be mutually exclusive (Furgason et al., 2009). In other words, the results obtained by Furgason et al. suggest that, Vps45p interacts either with the N-peptide motif or with the closed conformation of Tlg2p, but not with both simultaneously. This is different to the results by Burkhardt et al. that suggest that both binding sites can be used simultaneously. Therefore, there might be an allosteric coupling between the two spatially separated binding sites on the SM protein, that could vary, perhaps according to the needs of the organism or of the trafficking step. It would also be interesting to know whether the same SM protein can bind to different syntaxins in a distinct manner. For instance, Sly1p binds to another syntaxin Ufe1p on the retrograde pathway between the ER and Golgi (Yamaguchi et al., 2002). Sly1p is thought to interact only with the N-peptide motif of Ufe1p. I was able to confirm this binding mode in this study, but could not carry out further experiments, since I could not purify the complete cytosolic portion of Ufe1p in the monomeric form. Nevertheless, $\mathrm{N}$ - or C-terminally truncated variants of Ufe1p should be tested in ITC experiments for their binding abilities to Sly1p in the future.

Assessments based on qualitative binding assays between SM proteins and syntaxins could not expose second binding sites and this might sometimes lead to misinterpretations of the findings obtained by in vivo trafficking studies. For instance, previous studies suggested that Tlg2p uses only its N-peptide for binding to Vps45p (Dulubova et al., 2002, Carpp et al., 2006). However, these studies either did not test the appropriate truncated constructs or were based on in vitro pulldown of recombinant proteins with their tagged binding partners. In one study, Vps45p-L117R mutant, which was shown to be unable to bind the N-peptide of Tlg2p, was expressed in yeast as the sole copy of Vps45p and no trafficking defects were observed (Carpp et al., 2006). Consequently, the N-peptide binding mode of Vps45p/Tlg2p pair was thought to play a mere role in secretion, perhaps only for the recruitment of Vps45p to the sites of secretion. However, the recent study by Furgason and co-workers has demonstrated that Vps45pTlg2p interaction can only be disturbed by abrogation of both the N-peptide and the closed conformation binding sites (Furgason et al., 2009), thus possibly explaining the lack of a trafficking defect of the Vps45p-L117R mutant. 
Based on qualitative binding assays, Sly1p has also been reported to bind only the Npeptide motif of Sed5p (Grabowski and Gallwitz, 1997, Kosodo et al., 1998, Yamaguchi et al., 2002). It has been shown by in vitro pulldown assays, that the F10A mutation of Sed5p completely abolishes the N-peptide interaction and thus the high-affinity Sly1p/Sed5p binding (Yamaguchi et al., 2002, Peng and Gallwitz, 2004). Akin to the example above, when Sed5p-F10A mutant was expressed in yeast as the sole copy of Sed5p, no effect was observed in cell growth, in the localization of Sly1p or in the vesicular protein transport (Peng and Gallwitz, 2004). The interpretation was that Sly1p/Sed5p interaction is dispensible for vesicular trafficking. Since my results identified a previously uncharacterized binding site between Sly1p and Sed5p, I tested whether the F10A mutant is unable to bind Sly1p. I titrated Sly1p with either Sed5p F10A (1-21) or Sed5p F10A (1320) using ITC. Sed5p F10A (1-21), containing only the N-peptide, did not bind Sly1p, confirming the results obtained by Peng and co-workers (Peng and Gallwitz, 2004). However, Sed5p F10A (1-320), containing the entire cytosolic portion of Sed5p, bound Sly1p, but with a clearly reduced enthalpy $(\Delta \mathrm{H} \approx 9 \mathrm{kcal} /$ mole $)$ and affinity $\left(\mathrm{K}_{\mathrm{d}} \approx 234 \mathrm{nM}\right)$ compared to wild-type. This finding confirms the critical importance of the N-peptide binding for the high-affinity Sly1p/Sed5p interaction, however, it reveals that the two proteins still interact when $\mathrm{N}$-peptide binding is severely disturbed. This suggests that the F10A mutant used in the in vivo study above is still able to function, and it still binds to Sly1p.

\subsection{Assembly of the ER-Golgi SNAREs}

Biophysical studies revealed that individual SNARE motifs are largely unstructured and major conformational changes occur upon their assembly (reviewed in Fasshauer, 2003). Using CD spectroscopy, interactions within the neuronal and endosomal SNARE subunits have been followed (Fasshauer et al., 1998a, Antonin et al., 2000, Zwilling et al., 2007), revealing formation of partial complexes between the subunits. Folding-unfolding hysteresis observed in SNARE complexes, suggests that SNARE assembly requires an intermediate, which could be detected in the CD experiments in some cases (Fasshauer et al., 1998a, Fasshauer et al., 2002). Therefore, I monitored the interactions between the ERGolgi SNAREs using CD spectroscopy to reveal the putative intermediate of their assembly pathway. 
Here, I aimed to use only the SNARE motifs of Sed5p (Qa), Bos1p (Qb), Bet1p (Qc) and Sec22p (R) in CD experiments, however, the entire cytosolic portion of Bet1p was used instead, since the protein was instable in the absence of its $\mathrm{N}$-terminal portion. CD spectroscopy revealed that all of the individual SNAREs were unstructured, similar to the other SNARE motifs that have been previously studied (Rice et al., 1997, Fasshauer et al., 1998a, Antonin et al., 2000, Zwilling et al., 2007). Upon mixing of all four SNAREs, a striking increase in the a-helical content was observed, indicating the formation of the SNARE complex. I mixed all combinations of individual proteins and collected their far UV-CD spectra in order to observe what complexes could form between the ER-Golgi SNAREs. In this way, a binary interaction between Sed5p (Qa) and Sec22p (R) was identified, as inferred from an increase in a-helical content. Interestingly, major structural changes were observed only upon mixing three SNARE motifs at a time. Three out of four combinations, i.e. potential ternary complexes, showed major structural changes. Interestingly, each of the three ternary combinations included Sed5p (Qa). Furthermore, the ternary combinations of Qabc-, QabR- and QacR-SNAREs were found to form stable complexes, since it was possible to purify them chromatographically. Hence, by CD experiments, I obtained a list of putative intermediates for SNARE complex formation.

Using CD spectroscopy, I also followed the assembly kinetics of SNAREs by the change of the CD signal at $222 \mathrm{~nm}$ over time. Assembly of the QabcR quaternary complex, as well as the Qabc, QabR and QacR ternary complexes were monitored. In each case, assembly was slow (about $\leq 50 \%$ assembly within an hour, when $2 \mu \mathrm{M}$ SNAREs were used). Notably assembly of the QabR complex was the fastest. Assembly of the quaternary complex was followed either using the sole SNARE motif (QaH3: residues 211-320) or the entire cytosolic portion of Sed5p (Qafull: residues 1-320). Strikingly, the quaternary SNARE complex did not form within the time of the experiment (about an hour), when Qafull was used. This suggested that Sed5p exists in a closed conformation, which interferes strongly with SNARE assembly. Nevertheless, the N-terminal domain of Sed5p is already folded and this could have also decreased the percentage of observable change of the CD signal. More direct evidence for the idea that the closed conformation of Sed5 slows SNARE complex formation came later from the experiments, which employed fluorescence anisotropy to follow the assembly kinetics.

In order to monitor the speed of SNARE complex formation using fluorescence anisotropy, Bos1p (Qb), Bet1p (Qc) and Sec22p (R) single-Cysteine variants were 
generated and labeled with fluorophores. Each of the fluorescent proteins was mixed with their respective unlabeled partner SNAREs to follow SNARE complex formation by the increase in fluorescence anisotropy as read-out. Two approaches were used for performing the anisotropy experiments. The first approach involved simultaneous mixing of the labeled and unlabeled SNAREs immediately before starting the measurements. This approach was used to get a general impression about the assembly kinetics of the SNAREs, without gaining insights into the assembly mechanism. Concurrent with the CD experiments, SNARE assembly was slower in each experiment where the Qafull was used instead of the QaH3. This finding strongly supported the notion that individual Sed5p preferentially exists in a closed state, which retards SNARE assembly. The second approach was developed to understand which putative intermediates serve during SNARE complex formation. For this, I pre-incubated the unlabeled SNAREs and thereby promoted formation of partial complexes, prior to mixing them with the fluorescent SNARE. Pre-incubation of Qa-, Qb-and Qc-SNAREs as well as Qa-, Qc- and R-SNAREs did not enhance the kinetics of the SNARE complex formation, implicating that subcomplexes, which form in those combinations, do not support SNARE assembly. Strikingly, pre-incubation of $\operatorname{Sed5p}(\mathrm{Qa}), \operatorname{Bos} 1 \mathrm{p}(\mathrm{Qb})$ and $\operatorname{Sec} 22 \mathrm{p}(\mathrm{R})$ allowed rapid binding of the fluorescent-Bet1p (Qc), strongly suggesting that an active folding intermediate forms between the Qa-, Qb- and R-SNAREs. In contrast, a purified complex of the QabR-SNAREs did not serve as a fast binding site. Therefore, the QabR-folding intermediate seems to exist in a transient state and is not a stable entity.

Notably, a previous study indirectly supports the notion that a QabR-intermediate could serve during SNARE complex formation. In this study, the ER-Golgi SNAREs were reconstituted into two populations of synthetic liposomes, with the individual SNARE proteins distributed in all possible combinations between them. Fusion of liposomes was observed only upon a single topological distribution of the SNAREs; Sed5p, Bos1p and Sec22p reconstituted on one population of liposomes and Bet1p reconstituted on the other (Parlati et al., 2000). This observation is not extraordinarily surprising when one now considers my finding that an active subcomplex for SNARE assembly forms only upon mixing Sed5p, Bos1p and Bet1p. Reconstitution of all three SNAREs on the same liposome possibly enhanced formation of the active subcomplex, and thus also the SNAREmediated liposome fusion. Indeed, based on this (Parlati et al., 2000) and subsequent studies (Parlati et al., 2002), it was claimed that fusion only occurs when SNAREs are 
confined to the two membrane compartments in a specific composition. According to this model, only a specific topological relationship of the linker regions, between the SNARE motifs and the membrane anchors, leads to a force transduction that allows membrane fusion. However, this interpretation should be considered with precaution, since distribution of SNAREs in different combinations on liposomes could also enhance or limit formation of putative SNARE intermediates. Furthermore, membrane localization of ER-Golgi SNAREs, except for the Golgi-resident Sed5p (Hardwick and Pelham, 1992, Banfield et al., 1994), is not well-known. Hence, Bos1p and/or Sec22p could also reside on the ER-derived vesicle.

Anisotropy experiments revealed that a transient QabR-subcomplex allows rapid assembly of ER-Golgi SNAREs, however, the stoichiometry and the structural features of this complex are unknown. Some evidence suggests that a partial complex between the Qa- and R-SNAREs could initiate the assembly of the QabR-subcomplex. For instance, I observed a binary interaction between Sed5p and Sec22p using CD spectroscopy. Moreover, pre-incubation of Sed5p and Sec22p seems to form a partial complex, which is ready to accept specifically Bos1p, but not Bet1p (see Appendix A.3). However, it is also plausible that Qa-, Qb- and R-SNAREs assemble by a single reaction.

Experiments performed by pre-mixing Qa-, Qb- and R-SNAREs at different concentrations (see Figure 3.17) demonstrated that the transient QabR-intermediate exists in a dynamic equilibrium with its constituents. These experiments also indicated that QabR mixes containing the Qafull are less stable compared to the mixes containing the QaH3. This effect is more prominent when the mixes are at lower concentrations in the assembly reactions. What could be the reason for that? In a possible scenario, the Nterminal domain of Sed5p competes with the Qb- and R-SNAREs to bind the SNARE motif of Sed5p. When the Qb- and R-SNAREs are present at lower concentrations, the Nterminal domain of Sed5p could compete better, since its local concentration relative to the SNARE motif is constant. This model could be tested by addition of the QaH3-QbR mixes together with the N-terminal domain of Sed5p (Sed5p 1-210) in the SNARE assembly reactions.

QabR subcomplexes seem to serve during ER-Golgi SNARE assembly, but they have also been suggested to serve during recognition of ER-derived vesicles in vivo (Mossessova et al., 2003). Since COPII coats of ER-derived vesicles are possibly not entirely shed when 
they reach to the Golgi (Cai et al., 2007), they are thought to have a potential role in vesicle tethering/docking. It was suggested that COPII coats of ER-derived vesicles can preferentially select the fusogenic forms of the SNAREs, thereby program vesicles for fusion (Mossessova et al., 2003, Lee et al., 2004). According to one study, Sed5p, Bet1p and Sec22 $p$ are recruited to COPII vesicles via recognition of discrete peptide motifs residing right at the N-termini of their SNARE motifs. These peptide motifs are either occluded or exposed, depending whether the SNARE protein is isolated or in a complex with other SNAREs. Bet1p seems to be recruited to the vesicles in the monomeric form, since its binding motif was shown to be buried upon SNARE assembly (Mossessova et al., 2003). Interestingly, the binding motifs of Sed5p and Sec22p are found to be accessible when they are in a complex with Bos1p. Thus, according to the study by Mossessova et al., QabR subcomplexes might assemble before or during the vesicle recognition step to facilitate the subsequent steps for SNARE complex formation and fusion. This supports the SNARE assembly model, i.e. that QabR subcomplex can indeed serve as acceptor complex for Bet1p, obtained by my biophysical investigations.

In the present study, rapid SNARE assembly was observed also upon artificial stabilization of a Qabc-subcomplex (see Figure 3.15). However, the idea that a Qabcsubcomplex serves as a folding intermediate for the ER-Golgi SNAREs is not supported by experiments, in which the Qa-, Qb- and Qc-SNARE subunits were premixed. Thus, experiments performed with subcomplexes analogous to the neuronal $\Delta \mathrm{N}$-complex could be misleading, if there is no information in hand about the assembly mechanism of a particular SNARE complex. Exemplified by the ER-Golgi SNARE complex and endosomal SNARE complexes, the folding intermediate of a SNARE complex may not always be in Qabc composition. When all combinations of individual early endosomal SNAREs are mixed, partial complexes form only in QacR- or QbcR compositions as observed by CD spectroscopy (Zwilling et al., 2007). Anisotropy experiments using a pre-mixing approach that I have used could reveal whether either or both of these partial complexes serve as intermediates. Interestingly, in the case of late endosomal SNAREs, no partial complexes was detectable by CD experiments (Antonin et al., 2000). However, the complexes could be only transient and perhaps could be obtained by a similar pre-mixing approach that I have used in this study. It would be important to know whether also the neuronal and yeast exocytic SNARE complexes could assemble via intermediates other than in Qabc composition. For instance, partial complexes in QabR and QacR compositions have been 
observed for neuronal SNAREs (Fasshauer et al., 1998a), but it has not been tested rigorously whether these complexes could serve during SNARE assembly.

As explained in section 1.4.1, the two R-SNAREs, Sec22p and Ykt6p, can functionally substitute for each other in ER-to-Golgi trafficking (Liu and Barlowe, 2002). I compared Sec22p and Ykt6p in SNARE assembly assays, in which I used the stabilized Qabcsubcomplexes as intermediates (see Figure 3.15). Sec22p bound to the $\Delta \mathrm{N}$-complexes rapidly, whereas Ykt6p was able to bind very slowly. Inferred from these experiments, the two R-SNAREs seem to differ in their SNARE binding properties. This observation is also consistent with a previous study, showing that Sec22p, but not Ykt6p can interact with the $\triangle$ N-complex of neuronal SNAREs (Wiederhold et al., 2010). It would be interesting to test whether a transient QabR-subcomplex could be assembled using Ykt6p instead of Sec22p and whether Bet1p could rapidly bind to this QabR-subcomplex. It might be possible that Ykt6 does not assemble into a comparable QabR-subcomplex, preferring a different SNARE assembly pathway. Notably, a different assembly pathway for the two R-SNAREs could allow for distinguishing two ER-Golgi trafficking steps by using an almost identical set of SNARE proteins.

\subsection{Regulation of the assembly of ER-Golgi SNAREs by Sly1p}

My findings indicate that individual Sed5p is preferentially in a closed conformation, slowing down the SNARE assembly. Since not only the N-peptide but also the C-terminal portion of Sed5p contributes to Sly1p binding, the structural configuration of Sed5p could be rearranged by the SM protein. Therefore, I investigated how Sly1p-bound Sed5p would act during SNARE assembly.

Initially I wanted to observe whether Sly1p had any effect on the speed of the SNARE complex formation. For this, Sly1p was included in the anisotropy experiments in which I did not pre-mix the ER-Golgi SNAREs. In this way, I observed that Sly1p has an accelerating effect on the SNARE complex formation. Since my previous experiments demonstrated that assembly of the ER-Golgi SNAREs involves a transient QabR intermediate, I next examined whether Sly1p supports the QabR intermediate or another subcomplex along the assembly pathway. For this, I included Sly1p in the pre-mixes of the ER-Golgi SNAREs. A significant effect on assembly kinetics of the SNAREs was 
observed only when Sly1p was pre-mixed with the Qa-, Qb- and R-SNAREs, indicating that Sly1p supports the QabR-intermediate. Conversely, when a purified complex of Sly1p, Sed5p, Bos1p and Sec22p was used in the reactions, assembly was slow. Thus, Sly1p apparently accelerates SNARE assembly through supporting the transient QabR intermediate. Further support for this interpretation came from FRET experiments which monitored the SNARE complex formation in the presence or in the absence of Sly1p (see Figure 3.22).

Although it was clear from my results that Sly1p supports a particular assembly pathway during SNARE complex formation, it was not clear how this occurs. Experiments, in which the premixes were used at lower concentrations, shed first light on this, showing that Sly1p stabilizes the QabR intermediate (see Figure 3.20). How Sly1p stabilizes this transient intermediate remains to be clarified, however. It is plausible to suggest that Sly1p simply accelerates formation of the QabR-subcomplex. However, anisotropy experiments following the formation of the QabR intermediate (see figure 3.21) showed that Sly1p does not significantly enhance the assembly kinetics of this intermediate. Thus, perhaps, Sly1p somewhat slows the dissociation of the QabR intermediate once it has been formed. But how could Sly1p do so? It is conceivable that Sly1p might simply grasp the N-terminal portion of Sed5p. As discussed in section 4.2, the N-terminal portion of Sed5p might otherwise compete with the $\mathrm{Qb}$ - and R-SNAREs for binding to the Sed5p SNARE motif (see figure 3.17). However, as the precise configuration of the QabR subcomplex remains unknown, this model is rather speculative. Structural studies would be required to precisely understand how Sly1p acts on the QabR-intermediate.

The high structural homology between SM proteins suggests a common molecular mechanism for their function. Therefore, further studies with quantitative readouts will be required to know whether other SM proteins can function similar to Sly1p. Structural and biochemical evidence indicates that neuronal Munc18a inhibits SNARE assembly by its interaction with Syntaxin 1a (Pevsner et al., 1994, Yang et al., 2000, Misura et al., 2000). However, physiological evidence suggests that Munc18a acts at multiple steps during synaptic vesicle fusion and somehow stimulates neurotransmission (Gulyas-Kovacs et al., 2007). In one possible scenario, Munc18a/Syntaxin 1a interaction initially blocks SNARE assembly to provide spatiotemporal control over exocytosis. When the block is relieved by a yet unknown mechanism, Munc18a could orchestrate SNARE assembly in a similar way to Sly1p. A similar experimental strategy used in this study could reveal whether 
Munc18a might stabilize the folding intermediate of the neuronal SNARE machinery. Since regulated secretion events would possibly require further organization compared to constitutive secretion events, it could be investigated whether Munc18a needs to work together with accessory proteins, such as tethering/docking or priming factors, to facilitate SNARE complex formation.

In contrast to all previously discussed SM proteins, the yeast exocytic Sec1p appears to be an exception since it is proposed to interact only with the assembled SNARE complexes but not with monomeric SNAREs (Togneri et al., 2006, Carr et al., 1999, Scott et al., 2004). Concurrent with this notion, Sec1p was suggested to play a late role in constitutive exocytosis, only after SNARE complex assembly (Carr et al., 1999, Grote et al., 2000b). Interestingly, a recent study has shown that Sec1p might function both before and after SNARE complex assembly (Hashizume et al., 2009). In this study, a diverse panel of yeast sec1 mutants were isolated and categorized into two classes. Class A mutants exhibit a tight block on vesicle docking and a defect in SNARE complex assembly, whereas Class B mutants have a SNARE complex binding defect with accompanying secretion defects. These observations raise the question of how can Sec1p function upstream of SNARE assembly, considering that it shows no interaction with Sso1p and also other yeast exocytic SNAREs. Recent evidence suggests that Sec1p interacts with accessory factors to regulate SNARE assembly. Indeed, it has been shown that Sec1p closely associates with a protein called Mso1p, as well as a Rab GTPase Sec4p and its GEF Sec2p at sites of secretion (Weber et al., 2010, Weber-Boyvat et al., 2011). It would be important to biochemically characterize the interactions between Sec1p and the associated factors since Sec1p perhaps interacts with monomeric Sso1p only in the presence of these factors. Sly1p was discovered via a genetic screen since a single amino acid substitution (E532K) of a dominant mutant, termed Sly1-20 (Śuppressor of Loss of $\underline{Y} p t 1$ function), was able to bypass the deletion of the Rab protein Ypt1p (Dascher et al., 1991). However, it is still unknown how Ypt1p and Sly1p are functionally coupled. Biochemical studies on the interactions between Sec1p, Sec4p and Sec2p might shed some light on how Ypt1p and Sly1p are functionally coupled as well. As exemplified by Sec1p and the HOPS complex, all SM proteins could be a part of a multiprotein machinery. The effect of Sly1p observed in SNARE assembly could be altered or promoted in the presence of Rabs and other factors. 
Two exocytitc SM proteins, the vertebrate Munc18a and the yeast Sec1p have been proposed to function on assembled SNARE complexes for promoting membrane fusion (Shen et al., 2007, Carr et al., 1999, Dulubova et al., 2007, Deak et al., 2009, Hashizume et al., 2009). The SNARE complex-Sec1p interaction has not been structurally and biochemically characterized yet, thus it is entirely unknown how this interaction may be involved in executing fusion. Interaction of Munc18a with the neuronal SNARE complex has been investigated, but biochemical data suggests that Munc18a binds to the four-helix SNARE bundle with low micromolar affinity (Xu et al., 2010), remaining associated with it via attaching the $\mathrm{N}$-peptide or the entire $\mathrm{N}$-terminal domain of Syntaxin 1a (Burkhardt et al., 2008). In this study, I have tested whether Sly1p can also bind the assembled ER-Golgi SNARE complex (see Figure 3.9). My findings suggest that Sly1p does not interact with the SNARE core complex. However, it should be noted that, ITC might not be well-suited to detect a very weak interaction between Sly1p and the four-helix SNARE bundle. The weak associations between SM proteins and the core region of SNARE complexes argue against models which propose that SM proteins act as "clasps" on the four-helical SNARE bundles. Hence, it is still largely unknown how SM proteins could function once the SNARE complex has been formed. Structural characterization of the SNARE complex-SM protein interactions will be required to shed some light on this problem.

\subsection{Do the two binding sites on Sly1p work together for regulating SNARE assembly?}

Inferred from the studies of Burkhardt et al., the two binding sites of the Munc18a/Syntaxin 1a pair collaborates during SNARE assembly (Burkhardt et al., 2008). When both binding sites are available, Munc18a seem to inhibit SNARE complex formation, whereas, the block is relieved when N-peptide is removed. Similarly, when a double mutation (L165A/E166A) is introduced on the linker region of Syntaxin 1a to destabilize its closed conformation, the mutant protein could still bind Munc18a, but is not rendered inaccessible for SNARE assembly anymore (Burkhardt et al., 2008). Therefore, depending on the binding status of Munc18a to the two distinct sites, the conformation of the Munc18a/Syntaxin 1a is altered such that the accessibility of the Syntaxin 1a SNARE motif is modulated. These findings suggest that Munc18a makes use of its both binding sites when regulating the neuronal SNARE assembly. 
If the two binding sites of the Sly1p/Sed5p pair collaborate in analogy to the Munc18a/Syntaxin 1a pair, regulation of SNARE assembly by Sly1p could be disturbed or altered upon mutating one of the two binding sites of Sed5p. In order to test this, the F10A mutant of Sed5p was used in the SNARE assembly assays in the presence or in the absence of Sly1p. It needs to be kept in mind that, the F10A mutation in yeast was demonstrated to have no effect on the transport kinetics of protein cargo between the ER and Golgi (Peng and Gallwitz, 2004). Hence, it would be important to know whether this mutation can severely affect the regulation of Sly1p on SNARE assembly or not. The F10A mutant was used in two types of SNARE assembly assays using either anisotropy or FRET approach (see Figure 3.23). Somewhat different results were obtained upon using the two different approaches. Using the anisotropy approach, only a slight decrease was observed in the promoting effect of Sly1p on SNARE assembly. However, a severe defect was observed using the FRET approach, very likely due to the overall lower SNARE protein concentrations used in the experiments, and thus due to a reduced likelihood for the formation of the QabR intermediate. One interpretation of these results could be that in conditions favoring the formation of the QabR intermediate, the role of the Sed5p Npeptide is perhaps less critical for regulation of SNARE assembly. Thus, perhaps it is not very surprising that the F10A mutant causes no trafficking defects in yeast, since intracellular conditions might favor formation of the QabR-intermediate through different factors, e.g., COPII coat or accessory proteins or lipids or spatial segregation of the SNAREs on membranes. Alternatively, there could be regulatory factors in yeast that are functionally redundant with the $\mathrm{N}$-peptide.

Since it was observed using the FRET approach that, the F10A mutant perturbs the SNARE complex-promoting activity of Sly1p, one could suggest that the two binds sites of Sly1p collaborate during SNARE assembly. About $5 \mu \mathrm{M}$ of the F10A mutant and about $7.5 \mu \mathrm{M}$ of Sly1p were used in the FRET assays, indicating that Sly1p and the F10A mutant could interact in the experimental conditions based on their binding affinity $\left(\mathrm{K}_{\mathrm{d}} \approx 234\right.$ $\mathrm{nM}$ ). Since the observed effect cannot be attributed to abolution of the Sly1p/Sed5p interaction, disruption of N-peptide binding must be involved. It is possible that when the $\mathrm{N}$-peptide binding is weakened, Sly1p cannot that easily render the SNARE motif of Sed5p accessible. FRET approach might allow to observe this effect better compared to anisotropy approach, since assembly of the QabR-intermediate and thus, the SNARE complex, might be less efficient due to the low amount of the fluorescent R-SNARE $(\sim 50$ 
$\mathrm{nM}$ ) used in the FRET experiments. Supporting the notion that N-peptide plays a critical role, the effect of Sly1p was completely abolished in the presence of $\Delta \mathrm{N}$-Sed5p, lacking the N-peptide, no matter whether the anisotropy or FRET approaches were used (see Figure 3.24). However, in this case, it cannot be ruled out that Sly1p and Sed5p interaction was not abolished. There was no detectable binding between Sly1p and $\Delta \mathrm{N}$-Sed5p using ITC, thus, it might be possible that Sly1p could not bind $\Delta \mathrm{N}-\mathrm{Sed} 5 \mathrm{p}$ in experimental conditions and thereby could not orchestrate SNARE assembly.

In addition to the N-peptide, Sly1p interacts with the C-terminal part of Sed5p, possibly in a closed conformation. I wanted to introduce mutations in Sed5p to destabilize its closed conformation in order to observe whether these mutations would alter the SNARE complex-promoting activity of Sly1p. I designed mutations which are in analogy to the ones that were previously introduced in Sso1p (Munson et al., 2000). However, none of the mutations destabilized the closed conformation drastically as monitored by SNARE assembly assays. Then, I replaced the entire linker region with a flexible linker and generated the mutant protein Sed5p-linked in order to observe whether Sly1p requires the linker region to promote SNARE assembly. Interestingly, Sly1p effect on SNARE assembly remained unperturbed in the presence of Sed5p-linked, suggesting that Sly1p does not modulate the linker region during SNARE complex formation. Thus, it remains unclear how Sly1p could rearrange the structural configuration of Sed5p using the two binding sites. It is also possible that the coupling between the two binding pockets of Sly1p is not primarily required for rearranging the configuration of Sed5p, but is rather needed for modulating configuration and/or stability of the folding intermediate. 


\section{Chapter 5}

\section{Summary \& Conclusions}

SM proteins and SNAREs are two integral components of vesicle fusion in the secretory pathway. According to current thinking, SNAREs present in opposing membranes assemble into a four-helical bundle, thereby pulling the membranes in close proximity to facilitate fusion. SM proteins are thought to exert their function by interacting with the SNARE machinery, primarily with the syntaxins (Qa-SNAREs). Despite the highly conserved structural properties of both SM proteins and syntaxins, different binding modes have been proposed for their association. Neuronal Munc18a grasps and stabilizes a closed conformation of Syntaxin 1a, whereas several other SM proteins are thought to interact with a short $\mathrm{N}$-peptide motif of their cognate syntaxins. Recent findings suggest that there can be a common binding mode between SM proteins and syntaxins, involving both the N-peptide and the closed conformation. Furthermore, exemplified by the action of Munc18a on SNARE assembly, SM proteins could make use of both binding sites for executing their regulatory function.

The yeast SM protein Sly1p tightly interacts with the syntaxin Sed5p in the ER-to-Golgi transport step. However, it is undefined whether Sly1p/Sed5p pair could orchestrate SNARE assembly. In light of the new findings, I aimed to deeply investigate the Sly1p/Sed5p interaction and its impact on the SNARE complex formation. Previous analysis demonstrated that Sly1p interacts with the short N-terminal peptide motif of Sed5p, but a second binding site for the Sly1p/Sed5p pair was not ruled out. Initially I wanted to find out whether individual Sed5p can adopt a closed conformation, since it might contribute to Sly1p binding. Using CD spectroscopy, I observed that the N-terminal portion of Sed5p is significantly stabilized by the SNARE motif of the syntaxin. In 
addition, using native gel electrophoresis, size exclusion chromatography and ITC, I detected an interaction between the N-terminal portion and the SNARE motif of Sed5p. These observations suggested that Sed5p can adopt a closed conformation. Afterwards, I re-examined the Sly1p/Sed5p interaction using biochemical and biophysical tools. ITC experiments revealed that the $\mathrm{N}$-peptide motif is the major contributor to the interaction, but also the remaining region of Sed5p, possibly in a closed conformation, binds Sly1p. Further biophysical characterization involving fluorescence anisotropy and EPR spectroscopy supported this notion.

The assembly of ER-Golgi SNAREs and the dynamics of their interactions were not studied before. I investigated the assembly mechanism of the ER-Golgi SNARE machinery, to be able to fully understand the action of Sly1p. Using CD spectroscopy, I identified the partial complexes which could form between the SNAREs. The ternary combinations of Qabc-, QabR- and QacR-SNAREs formed complexes, observed using CD spectroscopy and also chromatographically. Since studies on different SNARE complexes suggest that the SNARE complex formation proceeds through an intermediate, I wanted to figure out whether one of the subcomplexes formed between the ER-Golgi SNAREs could be a folding intermediate. I developed the tools to monitor the assembly kinetics of SNAREs and later, I designed kinetics assays in which I promoted formation of the putative intermediate complexes during SNARE complex formation. I observed that only a subcomplex formed between Qa-, Qb- and R-SNAREs allows rapid SNARE assembly, suggesting that the QabR-subcomplex could be the folding intermediate for SNARE complex formation. Later, I found out that the QabR-intermediate is a transient entity and it exists in a dynamic equilibrium with its constituents.

In order to test whether Sly1p has an effect on SNARE complex formation, I developed kinetic assays, in which I monitored the assembly kinetics of SNAREs in the presence and absence of Sly1p. I observed that Sly1p accelerates SNARE complex formation by supporting the transient QabR-intermediate. Preliminary data suggests that Sly1p could stabilize the QabR-subcomplex by slowing its dissociation, however, further work is needed to validate this notion. Finally, I wanted to figure out whether Sly1p acts on the two binding sites of Sed5p to orchestrate the SNARE machinery. I observed that mutations in the N-peptide region of Sed5p interfere with the SNARE complex-promoting activity of Sly1p, suggesting that N-peptide is required during regulation of SNARE assembly. 
My findings support the notion that there could be a common binding mode between SM proteins and syntaxins, involving both the $\mathrm{N}$-peptide and the closed conformation of the syntaxin. Sly1p and possibly other SM proteins could regulate the SNARE assembly by acting on the two binding sites, in a yet undefined manner. Sly1p/Sed5p pair seems to orchestrate a cascade of events that promote formation of a folding intermediate required for SNARE complex formation. Further biophysical studies are needed to answer whether other SM proteins could also have such a positive regulatory role. 


\section{Bibliography}

AALTO, M. K., RONNE, H., KERANEN, S. 1993. Yeast syntaxins Sso1p and Sso2p belong to a family of related membrane proteins that function in vesicular transport. EMBO J, 12, 4095, 104.

AN, S. J. \& ALMERS, W. 2004. Tracking SNARE complex formation in live endocrine cells. Science, $306,1042-6$.

ANTONIN, W., FASSHAUER, D., BECKER, S., JAHN, R. \& SCHNEIDER, T. R. 2002. Crystal structure of the endosomal SNARE complex reveals common structural principles of all SNAREs. Nat Struct Biol, 9, 107-11.

ANTONIN, W., HOLROYD, C., FASSHAUER, D., PABST, S., VON MOLLARD, G. F. \& JAHN, R. 2000. A SNARE complex mediating fusion of late endosomes defines conserved properties of SNARE structure and function. EMBO J, 19, 6453-64.

ARAC, D., DUlubOVA, I., PEI, J., HURYEVA, I., GRISHIN, N. V. \& RIZO, J. 2005. Threedimensional structure of the rSly1 N-terminal domain reveals a conformational change induced by binding to syntaxin 5. J Mol Biol, 346, 589-601.

ARAN, V., BRANDIE, F. M., BOYD, A. R., KANTIDAKIS, T., RIDEOUT, E. J., KELLY, S. M., GOULD, G. W. \& BRYANT, N. J. 2009. Characterization of two distinct binding modes between syntaxin 4 and Munc18c. Biochem J, 419, 655-60.

BALCH, W. E., DUNPHY, W. G., BRAELL, W. A. \& ROTHMAN, J. E. 1984. Reconstitution of the transport of protein between successive compartments of the Golgi measured by the coupled incorporation of N-acetylglucosamine. Cell, 39, 405-16.

BANFIELD, D. K., LEWIS, M. J., RABOUILLE, C., WARREN, G. \& PELHAM, H. R. 1994. Localization of Sed5, a putative vesicle targeting molecule, to the cis-Golgi network involves both its transmembrane and cytoplasmic domains. J Cell Biol, 127, 357-71.

BAUMERT, M., MAYCOX, P. R., NAVONE, F., DE CAMILLI, P. \& JAHN, R. 1989. Synaptobrevin: an integral membrane protein of 18,000 daltons present in small synaptic vesicles of rat brain. EMBO J, 8, 379-84.

BECHERER, K. A., JONES., E. W. 1992. EMBL Databank Rel., 31, M90395. 
BECKERS, C. J., BLOCK, M. R., GLICK, B. S., ROTHMAN, J. E. \& BALCH, W. E. 1989. Vesicular transport between the endoplasmic reticulum and the Golgi stack requires the NEM-sensitive fusion protein. Nature, 339, 397-8.

BENNETT, M. K., CALAKOS, N. \& SCHELLER, R. H. 1992. Syntaxin: a synaptic protein implicated in docking of synaptic vesicles at presynaptic active zones. Science, 257, 255-9.

BENNETT, M. K., GARCIA-ARRARAS, J. E., ELFERINK, L. A., PETERSON, K., FLEMING, A. M., HAZUKA, C. D. \& SCHELLER, R. H. 1993. The syntaxin family of vesicular transport receptors. Cell, 74, 863-73.

BERLINER, L. J., GRUNWALD, J., HANKOVSZKY, H. O. \& HIDEG, K. 1982. A novel reversible thiol-specific spin label: papain active site labeling and inhibition. Anal Biochem, 119, 450-5.

BLASI, J., CHAPMAN, E. R., LINK, E., BINZ, T., YAMASAKI, S., DE CAMILLI, P., SUDHOF, T. C., NIEMANN, H. \& JAHN, R. 1993. Botulinum neurotoxin A selectively cleaves the synaptic protein SNAP-25. Nature, 365, 160-3.

BONIFACINO, J. S., GLICK, B. S., 2004. The mechanisms of vesicle budding and fusion. Cell, 116, $153-66$.

BORISOVSKA, M., ZHAO, Y., TSYTSYURA, Y., GLYVUK, N., TAKAMORI, S., MATTI, U., RETTIG, J., SUDHOF, T. \& BRUNS, D. 2005. v-SNAREs control exocytosis of vesicles from priming to fusion. EMBO J, 24, 2114-26.

BRADFORD, M. M. 1976. A rapid and sensitive method for the quantitation of microgram quantities of protein utilizing the principle of protein-dye binding. Anal Biochem, 72, 248-54.

BRACHER, A., DRESBACH, T., BETZ, H. \& WEISSENHORN, W. 2000. Crystallization and preliminary X-ray analysis of squid neuronal Sec1. Acta Crystallogr D Biol Crystallogr, 56, 501-3.

BRACHER, A. \& WEISSENHORN, W. 2002. Structural basis for the Golgi membrane recruitment of Sly1p by Sed5p. EMBO J, 21, 6114-24.

BRANDHORST, D., ZWILLING, D., RIZZOLI, S. O., LIPPERT, U., LANG, T. \& JAHN, R. 2006. Homotypic fusion of early endosomes: SNAREs do not determine fusion specificity. Proc Natl Acad Sci U S A, 103, 2701-6. 
BRAUN, S. \& JENTSCH, S. 2007. SM-protein-controlled ER-associated degradation discriminates between different SNAREs. EMBO Rep, 8, 1176-82.

BRENNER, S. 1974. The genetics of Caenorhabditis elegans. Genetics, 77, 71-94.

BROCKER, C., ENGELBRECHT-VANDRE, S. \& UNGERMANN, C. 2010. Multisubunit tethering complexes and their role in membrane fusion. Curr Biol, 20, R943-52.

BRUNGER, A. T., 2006. Structure and function of SNARE and SNARE-interacting proteins. Quarterly Reviews of Biophysics, 38, 1-47.

BRYANT, N. J. \& JAMES, D. E. 2001. Vps45p stabilizes the syntaxin homologue Tlg2p and positively regulates SNARE complex formation. EMBO J, 20, 3380-8.

BURKHARDT, P., HATTENDORF, D. A., WEIS, W. I. \& FASSHAUER, D. 2008. Munc18a controls SNARE assembly through its interaction with the syntaxin N-peptide. EMBO J, 27, 923-33.

BURKHARDT, P., STEGMANN, C. M., COOPER, B., KLOEPPER, T. H., IMIG, C., VAROQUEAUX, F., WAHL, M. C. \& FASSHAUER, D. 2011. Primordial neurosecretory apparatus identified in the choanoflagellate Monosiga brevicollis. Proc Natl Acad Sci U S A.

BURRI, L. \& LITHGOW, T. 2004. A complete set of SNAREs in yeast. Traffic, 5, 45-52.

BURRI, L., VARLAMOV, O., DOEGE, C. A., HOFMANN, K., BEILHARZ, T., ROTHMAN, J. E., SOLLNER, T. H. \& LITHGOW, T. 2003. A SNARE required for retrograde transport to the endoplasmic reticulum. Proc Natl Acad Sci U S A, 100, 9873-7.

CAI, H., YU, S., MENON, S., CAI, Y., LAZAROVA, D., FU, C., REINISCH, K., HAY, J. C. \& FERRO-NOVICK, S. 2007. TRAPPI tethers COPII vesicles by binding the coat subunit Sec23. Nature, 445, 941-4.

CAO, X., BALLEW, N. \& BARLOWE, C. 1998. Initial docking of ER-derived vesicles requires Uso1p and Ypt1p but is independent of SNARE proteins. EMBO J, 17, 2156-65.

CAO, X. \& BARLOWE, C. 2000. Asymmetric requirements for a Rab GTPase and SNARE proteins in fusion of COPII vesicles with acceptor membranes. J Cell Biol, 149, 55-66.

CARPP, L. N., CIUFO, L. F., SHANKS, S. G., BOYD, A. \& BRYANT, N. J. 2006. The Sec1p/Munc18 protein Vps45p binds its cognate SNARE proteins via two distinct modes. J Cell Biol, 173, 927-36. 
CARR, C. M., GROTE, E., MUNSON, M., HUGHSON, F. M. \& NOVICK, P. J. 1999. Sec1p binds to SNARE complexes and concentrates at sites of secretion. J Cell Biol, 146, 333-44.

CARR, C. M. \& RIZO, J. 2010. At the junction of SNARE and SM protein function. Curr Opin Cell Biol, 22, 488-95.

CHEN, X., LU, J., DULUBOVA, I. \& RIZO, J. 2008. NMR analysis of the closed conformation of syntaxin-1. J Biomol NMR, 41, 43-54.

CHEN, Y. A., SCALES, S. J. \& SCHELLER, R. H. 2001. Sequential SNARE assembly underlies priming and triggering of exocytosis. Neuron, 30, 161-70.

CHERNOMORDIK, L. V. \& KOZLOV, M. M. 2003. Protein-lipid interplay in fusion and fission of biological membranes. Annu Rev Biochem, 72, 175-207.

CHERNOMORDIK, L. V. \& KOZLOV, M. M. 2008. Mechanics of membrane fusion. Nat Struct Mol Biol, 15, 675-83.

CHERNOMORDIK, L. V., ZIMMERBERG, J. \& KOZLOV, M. M. 2006. Membranes of the world unite! J Cell Biol, 175, 201-7.

CLARY, D. O., GRIFF, I. C. \& ROTHMAN, J. E. 1990. SNAPs, a family of NSF attachment proteins involved in intracellular membrane fusion in animals and yeast. Cell, 61, 709-21.

COLLINS, K. M. \& WICKNER, W. T. 2007. Trans-SNARE complex assembly and yeast vacuole membrane fusion. Proc Natl Acad Sci U S A, 104, 8755-60.

COWLES, C. R., EMR, S. D. \& HORAZDOVSKY, B. F. 1994. Mutations in the VPS45 gene, a SEC1 homologue, result in vacuolar protein sorting defects and accumulation of membrane vesicles. $J$ Cell Sci, 107 ( Pt 12), 3449-59.

D'ANDREA-MERRINS, M., CHANG, L., LAM, A. D., ERNST, S. A. \& STUENKEL, E. L. 2007. Munc18c interaction with syntaxin 4 monomers and SNARE complex intermediates in GLUT4 vesicle trafficking. J Biol Chem, 282, 16553-66.

DASCHER, C., MATTESON, J. \& BALCH, W. E. 1994. Syntaxin 5 regulates endoplasmic reticulum to Golgi transport. J Biol Chem, 269, 29363-6. 
DASCHER, C., OSSIG, R., GALLWITZ, D. \& SCHMITT, H. D. 1991. Identification and structure of four yeast genes (SLY) that are able to suppress the functional loss of YPT1, a member of the RAS superfamily. Mol Cell Biol, 11, 872-85.

DEAK, F., XU, Y., CHANG, W. P., DULUBOVA, I., KHVOTCHEV, M., LIU, X., SUDHOF, T. C. \& RIZO, J. 2009. Munc18-1 binding to the neuronal SNARE complex controls synaptic vesicle priming. J Cell Biol, 184, 751-64.

DEMIRCIOGLU, F. E., 2008. "Comparative biochemical and biophysical analysis of Sly1p/ Sed5p interaction and its effect on SNARE assembly", MSc thesis, University of Göttingen.

DIAZ, R., MAYORGA, L. S., WEIDMAN, P. J., ROTHMAN, J. E. \& STAHL, P. D. 1989. Vesicle fusion following receptor-mediated endocytosis requires a protein active in Golgi transport. Nature, 339, 398-400.

DIETRICH, L. E., BOEDDINGHAUS, C., LAGRASSA, T. J. \& UNGERMANN, C. 2003. Control of eukaryotic membrane fusion by N-terminal domains of SNARE proteins. Biochim Biophys Acta, $1641,111-9$.

DILCHER, M., VEITH, B., CHIDAMBARAM, S., HARTMANN, E., SCHMITT, H. D. \& FISCHER VON MOLLARD, G. 2003. Use1p is a yeast SNARE protein required for retrograde traffic to the ER. EMBO J, 22, 3664-74.

DULUbOVA, I., KHVOTCHEV, M., LIU, S., HURYEVA, I., SUDHOF, T. C. \& RIZO, J. 2007. Munc18-1 binds directly to the neuronal SNARE complex. Proc Natl Acad Sci U S A, 104, 2697-702.

DULUBOVA, I., SUGITA, S., HILL, S., HOSAKA, M., FERNANDEZ, I., SUDHOF, T. C. \& RIZO, J. 1999. A conformational switch in syntaxin during exocytosis: role of munc18. EMBO J, 18, 4372-82.

DULUBOVA, I., YAMAGUCHI, T., ARAC, D., LI, H., HURYEVA, I., MIN, S. W., RIZO, J. \& SUDHOF, T. C. 2003. Convergence and divergence in the mechanism of SNARE binding by Sec1/Munc18-like proteins. Proc Natl Acad Sci U S A, 100, 32-7.

DULUBOVA, I., YAMAGUCHI, T., GAO, Y., MIN, S. W., HURYEVA, I., SUDHOF, T. C. \& RIZO, J. 2002. How Tlg2p/syntaxin 16 'snares' Vps45. EMBO J, 21, 3620-31.

DULUBOVA, I., YAMAGUCHI, T., WANG, Y., SUDHOF, T. C. \& RIZO, J. 2001. Vam3p structure reveals conserved and divergent properties of syntaxins. Nat Struct Biol, 8, 258-64. 
FASSHAUER, D. 2003. Structural insights into the SNARE mechanism. Biochim Biophys Acta, 1641, 87-97.

FASSHAUER, D., ANTONIN, W., MARGITTAI, M., PABST, S. \& JAHN, R. 1999. Mixed and noncognate SNARE complexes. Characterization of assembly and biophysical properties. J Biol Chem, 274, 15440-6.

FASSHAUER, D., ANTONIN, W., SUBRAMANIAM, V. \& JAHN, R. 2002. SNARE assembly and disassembly exhibit a pronounced hysteresis. Nat Struct Biol, 9, 144-51.

FASSHAUER, D., BRUNS, D., SHEN, B., JAHN, R. \& BRUNGER, A. T. 1997a. A structural change occurs upon binding of syntaxin to SNAP-25. J Biol Chem, 272, 4582-90.

FASSHAUER, D., ELIASON, W. K., BRUNGER, A. T. \& JAHN, R. 1998a. Identification of a minimal core of the synaptic SNARE complex sufficient for reversible assembly and disassembly. Biochemistry, 37, 10354-62.

FASSHAUER, D. \& MARGITTAI, M. 2004. A transient N-terminal interaction of SNAP-25 and syntaxin nucleates SNARE assembly. J Biol Chem, 279, 7613-21.

FASSHAUER, D., OTTO, H., ELIASON, W. K., JAHN, R. \& BRUNGER, A. T. 1997b. Structural changes are associated with soluble N-ethylmaleimide-sensitive fusion protein attachment protein receptor complex formation. J Biol Chem, 272, 28036-41.

FASSHAUER, D., SUTTON, R. B., BRUNGER, A. T. \& JAHN, R. 1998b. Conserved structural features of the synaptic fusion complex: SNARE proteins reclassified as Q- and R-SNAREs. Proc Natl Acad Sci U S A, 95, 15781-6.

FERRO-NOVICK, S. \& JAHN, R. 1994. Vesicle fusion from yeast to man. Nature, 370, 191-3.

FIEBIG, K. M., RICE, L. M., POLLOCK, E. \& BRUNGER, A. T. 1999. Folding intermediates of SNARE complex assembly. Nat Struct Biol, 6, 117-23.

FISHER, R. J., PEVSNER, J. \& BURGOYNE, R. D. 2001. Control of fusion pore dynamics during exocytosis by Munc18. Science, 291, 875-8.

FORAN, P., SHONE, C. C. \& DOLLY, J. O. 1994. Differences in the protease activities of tetanus and botulinum $B$ toxins revealed by the cleavage of vesicle-associated membrane protein and various sized fragments. Biochemistry, 33, 15365-74. 
FURGASON, M. L., MACDONALD, C., SHANKS, S. G., RYDER, S. P., BRYANT, N. J. \& MUNSON, M. 2009. The N-terminal peptide of the syntaxin Tlg2p modulates binding of its closed conformation to Vps45p. Proc Natl Acad Sci U S A, 106, 14303-8.

GARCIA, E. P., GATTI, E., BUTLER, M., BURTON, J. \& DE CAMILLI, P. 1994. A rat brain Sec1 homologue related to Rop and UNC18 interacts with syntaxin. Proc Natl Acad Sci U S A, 91, 2003-7.

GENGYO-ANDO, K., KAMIYA, Y., YAMAKAWA, A., KODAIRA, K., NISHIWAKI, K., MIWA, J., HORI, I. \& HOSONO, R. 1993. The C. elegans unc-18 gene encodes a protein expressed in motor neurons. Neuron, 11, 703-11.

GENGYO-ANDO, K., KITAYAMA, H., MUKAIDA, M. \& IKAWA, Y. 1996. A murine neuralspecific homolog corrects cholinergic defects in Caenorhabditis elegans unc-18 mutants. J Neurosci, $16,6695-702$.

GERBER, S. H., RAH, J. C., MIN, S. W., LIU, X., DE WIT, H., DULUBOVA, I., MEYER, A. C., RIZO, J., ARANCILLO, M., HAMMER, R. E., VERHAGE, M., ROSENMUND, C. \& SUDHOF, T. C. 2008. Conformational switch of syntaxin-1 controls synaptic vesicle fusion. Science, 321, 1507-10.

GERST, J. E., RODGERS, L., RIGGS, M., WIGLER, M. 1992. SNC1, a yeast homolog of the synaptic vesicle-associated membrane protein/synaptobrevin gene family: genetic interactions with the RAS and CAP genes. Proc Natl Acad Sci U S A, 89, 4338-42.

GINGELL, D., GINSBERG, L. 1978. "Problems in physical interpretation of membrane interaction and fusion", in PASTE G., NICOLSON, G.L., Membrane fusion, Elsevier/North-Holland Biomedical Press, 791-833.

GIRAUDO, C. G., HU, C., YOU, D., SLOVIC, A. M., MOSHAROV, E. V., SULZER, D., MELIA, T. J. \& ROTHMAN, J. E. 2005. SNAREs can promote complete fusion and hemifusion as alternative outcomes. J Cell Biol, 170, 249-60.

GLICK, B. S. \& ROTHMAN, J. E. 1987. Possible role for fatty acyl-coenzyme A in intracellular protein transport. Nature, 326, 309-12.

GONZALEZ, L. C., JR., WEIS, W. I. \& SCHELLER, R. H. 2001. A novel snare N-terminal domain revealed by the crystal structure of Sec22b. J Biol Chem, 276, 24203-11. 
GORDON, D. E., BOND, L. M., SAHLENDER, D. A. \& PEDEN, A. A. 2010. A targeted siRNA screen to identify SNAREs required for constitutive secretion in mammalian cells. Traffic, 11, 1191204.

GRABOWSKI, R. \& GALLWITZ, D. 1997. High-affinity binding of the yeast cis-Golgi t-SNARE, Sed5p, to wild-type and mutant Sly1p, a modulator of transport vesicle docking. FEBS Lett, 411, $169-72$.

GROTE, E., BABA, M., OHSUMI, Y. \& NOVICK, P. J. 2000a. Geranylgeranylated SNAREs are dominant inhibitors of membrane fusion. J Cell Biol, 151, 453-66.

GROTE, E., CARR, C. M. \& NOVICK, P. J. 2000b. Ordering the final events in yeast exocytosis. J Cell Biol, 151, 439-52.

GULYAS-KOVACS, A., DE WIT, H., MILOSEVIC, I., KOCHUBEY, O., TOONEN, R., KLINGAUF, J., VERHAGE, M. \& SORENSEN, J. B. 2007. Munc18-1: sequential interactions with the fusion machinery stimulate vesicle docking and priming. J Neurosci, 27, 8676-86.

HALES, K. G. \& FULLER, M. T. 1997. Developmentally regulated mitochondrial fusion mediated by a conserved, novel, predicted GTPase. Cell, 90, 121-9.

HANSON, P. I., OTTO, H., BARTON, N. \& JAHN, R. 1995. The N-ethylmaleimide-sensitive fusion protein and alpha-SNAP induce a conformational change in syntaxin. J Biol Chem, 270, 16955-61.

HANSON, P. I., ROTH, R., MORISAKI, H., JAHN, R. \& HEUSER, J. E. 1997. Structure and conformational changes in NSF and its membrane receptor complexes visualized by quickfreeze/deep-etch electron microscopy. Cell, 90, 523-35.

HANSON, P. I. \& WHITEHEART, S. W. 2005. AAA+ proteins: have engine, will work. Nat Rev Mol Cell Biol, 6, 519-29.

HARDWICK, K. G. \& PELHAM, H. R. 1992. SED5 encodes a 39-kD integral membrane protein required for vesicular transport between the ER and the Golgi complex. J Cell Biol, 119, 513-21.

HASHIZUME, K., CHENG, Y. S., HUTTON, J. L., CHIU, C. H. \& CARR, C. M. 2009. Yeast Sec1p functions before and after vesicle docking. Mol Biol Cell, 20, 4673-85.

HATA, Y., SLAUGHTER, C. A. \& SUDHOF, T. C. 1993. Synaptic vesicle fusion complex contains unc-18 homologue bound to syntaxin. Nature, 366, 347-51. 
HATA, Y. \& SUDHOF, T. C. 1995. A novel ubiquitous form of Munc-18 interacts with multiple syntaxins. Use of the yeast two-hybrid system to study interactions between proteins involved in membrane traffic. J Biol Chem, 270, 13022-8.

HAZZARD, J., SUDHOF, T. C. \& RIZO, J. 1999. NMR analysis of the structure of synaptobrevin and of its interaction with syntaxin. J Biomol NMR, 14, 203-7.

HONG, W. 2005. SNAREs and traffic. Biochim Biophys Acta, 1744, 493-517.

HU, S. H., LATHAM, C. F., GEE, C. L., JAMES, D. E. \& MARTIN, J. L. 2007. Structure of the Munc18c/Syntaxin4 N-peptide complex defines universal features of the N-peptide binding mode of Sec1/Munc18 proteins. Proc Natl Acad Sci U S A, 104, 8773-8.

HUA, S. Y. \& CHARLTON, M. P. 1999. Activity-dependent changes in partial VAMP complexes during neurotransmitter release. Nat Neurosci, 2, 1078-83.

HUBBELL, W. L., CAFISO, D. S. \& ALTENBACH, C. 2000. Identifying conformational changes with site-directed spin labeling. Nat Struct Biol, 7, 735-9.

JAHN, R. \& GRUBMULLER, H. 2002. Membrane fusion. Curr Opin Cell Biol, 14, 488-95.

JAHN, R., LANG, T. \& SUDHOF, T. C. 2003. Membrane fusion. Cell, 112, 519-33.

JAHN, R. \& SCHELLER, R. H. 2006. SNAREs--engines for membrane fusion. Nat Rev Mol Cell Biol, 7, 631-43.

JOHNSON, J. R., FERDEK, P., LIAN, L. Y., BARCLAY, J. W., BURGOYNE, R. D. \& MORGAN, A. 2009. Binding of UNC-18 to the N-terminus of syntaxin is essential for neurotransmission in Caenorhabditis elegans. Biochem J, 418, 73-80.

KAISER, C. A. \& SCHEKMAN, R. 1990. Distinct sets of SEC genes govern transport vesicle formation and fusion early in the secretory pathway. Cell, 61, 723-33.

KHAN, A. H., THURMOND, D. C., YANG, C., CERESA, B. P., SIGMUND, C. D. \& PESSIN, J. E. 2001. Munc18c regulates insulin-stimulated glut4 translocation to the transverse tubules in skeletal muscle. J Biol Chem, 276, 4063-9.

KIENLE, N., KLOEPPER, T. H. \& FASSHAUER, D. 2009. Phylogeny of the SNARE vesicle fusion machinery yields insights into the conservation of the secretory pathway in fungi. BMC Evol Biol, 9, 19. 
KLOEPPER, T. H., KIENLE, C. N. \& FASSHAUER, D. 2007. An elaborate classification of SNARE proteins sheds light on the conservation of the eukaryotic endomembrane system. Mol Biol Cell, 18, 3463-71, <http:/ / bioinformatics.mpibpc.mpg.de/snare>.

KOSODO, Y., NODA, Y. \& YODA, K. 1998. Protein-protein interactions of the yeast Golgi t-SNARE Sed5 protein distinct from its neural plasma membrane cognate syntaxin 1. Biochem Biophys Res Commun, 250, 212-6.

KOZLOV, N. M., MARKIN, V. S. 1983. Possible mechanism of membrane fusion. Biofizika, 28, 24247.

KRAYNACK, B. A., CHAN, A., ROSENTHAL, E., ESSID, M., UMANSKY, B., WATERS, M. G. \& SCHMITT, H. D. 2005. Ds11p, Tip20p, and the novel Dsl3(Sec39) protein are required for the stability of the Q/t-SNARE complex at the endoplasmic reticulum in yeast. Mol Biol Cell, 16, 396377.

LAKOWICZ, J. R., 2000. Principles of Fluorescence Spectroscopy, Kluwer Academic, Plenum Publishers, New York.

LANG, T., MARGITTAI, M., HOLZLER, H. \& JAHN, R. 2002. SNAREs in native plasma membranes are active and readily form core complexes with endogenous and exogenous SNAREs. J Cell Biol, 158, 751-60.

LATHAM, C. F., LOPEZ, J. A., HU, S. H., GEE, C. L., WESTBURY, E., BLAIR, D. H., ARMISHAW, C. J., ALEWOOD, P. F., BRYANT, N. J., JAMES, D. E. \& MARTIN, J. L. 2006. Molecular dissection of the Munc18c/syntaxin4 interaction: implications for regulation of membrane trafficking. Traffic, 7, 1408-19.

LEE, M. C., MILLER, E. A., GOLDBERG, J., ORCI, L. \& SCHEKMAN, R. 2004. Bi-directional protein transport between the ER and Golgi. Annu Rev Cell Dev Biol, 20, 87-123.

LERMAN, J. C., ROBBLEE, J., FAIRMAN, R. \& HUGHSON, F. M. 2000. Structural analysis of the neuronal SNARE protein syntaxin-1A. Biochemistry, 39, 8470-9.

LEWIS, M. J., RAYNER, J. C. \& PELHAM, H. R. 1997. A novel SNARE complex implicated in vesicle fusion with the endoplasmic reticulum. EMBO J, 16, 3017-24.

LI, Y., GALLWITZ, D. \& PENG, R. 2005. Structure-based functional analysis reveals a role for the SM protein Sly1p in retrograde transport to the endoplasmic reticulum. Mol Biol Cell, 16, 3951-62. 
LI, Y., SCHMITT, H. D., GALLWITZ, D. \& PENG, R. W. 2007. Mutations of the SM protein Sly1 resulting in bypass of GTPase requirement in vesicular transport are confined to a short helical region. FEBS Lett, 581, 5698-702.

LIN, R. C. \& SCHELLER, R. H. 1997. Structural organization of the synaptic exocytosis core complex. Neuron, 19, 1087-94.

LIU, Y. \& BARLOWE, C. 2002. Analysis of Sec22p in endoplasmic reticulum/Golgi transport reveals cellular redundancy in SNARE protein function. Mol Biol Cell, 13, 3314-24.

LITTLETON, J. T., CHAPMAN, E. R., KREBER, R., GARMENT, M. B., CARLSON, S. D. \& GANETZKY, B. 1998. Temperature-sensitive paralytic mutations demonstrate that synaptic exocytosis requires SNARE complex assembly and disassembly. Neuron, 21, 401-13.

LODISH, H. F., BERK, A., MATSUDAIRA, P., KAISER, C., KRIEGER, M., SCOTT, M., ZIPURSKY, L., DARNELL., J. E. , 2004. Molecular Cell Biology, Sceintific American Press, New York.

LU, J., GARCIA, J., DULUBOVA, I., SUDHOF, T. C. \& RIZO, J. 2002. Solution structure of the Vam7p PX domain. Biochemistry, 41, 5956-62.

LUPAS, A., VAN DYKE, M. \& STOCK, J. 1991. Predicting coiled coils from protein sequences. Science, 252, 1162-4.

MALKA, F., GUILLERY, O., CIFUENTES-DIAZ, C., GUILLOU, E., BELENGUER, P., LOMBES, A. \& ROJO, M. 2005. Separate fusion of outer and inner mitochondrial membranes. EMBO Rep, 6, 8539.

MANCIAS, J. D. \& GOLDBERG, J. 2007. The transport signal on Sec22 for packaging into COPIIcoated vesicles is a conformational epitope. Mol Cell, 26, 403-14.

MARGITTAI, M., FASSHAUER, D., JAHN, R. \& LANGEN, R. 2003a. The Habc domain and the SNARE core complex are connected by a highly flexible linker. Biochemistry, 42, 4009-14.

MARGITTAI, M., FASSHAUER, D., PABST, S., JAHN, R. \& LANGEN, R. 2001. Homo- and heterooligomeric SNARE complexes studied by site-directed spin labeling. J Biol Chem, 276, 1316977 .

MARGITTAI, M., WIDENGREN, J., SCHWEINBERGER, E., SCHRODER, G. F., FELEKYAN, S., HAUSTEIN, E., KONIG, M., FASSHAUER, D., GRUBMULLER, H., JAHN, R. \& SEIDEL, C. A. 
2003b. Single-molecule fluorescence resonance energy transfer reveals a dynamic equilibrium between closed and open conformations of syntaxin 1. Proc Natl Acad Sci U S A, 100, 15516-21.

MARZ, K. E., LAUER, J. M. \& HANSON, P. I. 2003. Defining the SNARE complex binding surface of alpha-SNAP: implications for SNARE complex disassembly. J Biol Chem, 278, 27000-8.

MAYER, A. \& WICKNER, W. 1997. Docking of yeast vacuoles is catalyzed by the Ras-like GTPase Ypt7p after symmetric priming by Sec18p (NSF). J Cell Biol, 136, 307-17.

MCEWEN, J. M. \& KAPLAN, J. M. 2008. UNC-18 promotes both the anterograde trafficking and synaptic function of syntaxin. Mol Biol Cell, 19, 3836-46.

MCMAHON, H. T. \& SUDHOF, T. C. 1995. Synaptic core complex of synaptobrevin, syntaxin, and SNAP25 forms high affinity alpha-SNAP binding site. J Biol Chem, 270, 2213-7.

MCMAHON, H. T., USHKARYOV, Y. A., EDELMANN, L., LINK, E., BINZ, T., NIEMANN, H., JAHN, R. \& SUDHOF, T. C. 1993. Cellubrevin is a ubiquitous tetanus-toxin substrate homologous to a putative synaptic vesicle fusion protein. Nature, 364, 346-9.

MCNEW, J. A., PARLATI, F., FUKUDA, R., JOHNSTON, R. J., PAZ, K., PAUMET, F., SOLLNER, T. H. \& ROTHMAN, J. E. 2000. Compartmental specificity of cellular membrane fusion encoded in SNARE proteins. Nature, 407, 153-9.

MCNEW, J. A., SOGAARD, M., LAMPEN, N. M., MACHIDA, S., YE, R. R., LACOMIS, L., TEMPST, P., ROTHMAN, J. E. \& SOLLNER, T. H. 1997. Ykt6p, a prenylated SNARE essential for endoplasmic reticulum-Golgi transport. J Biol Chem, 272, 17776-83.

MCNEW, J. A., WEBER, T., ENGELMAN, D. M., SOLLNER, T. H. \& ROTHMAN, J. E. 1999. The length of the flexible SNAREpin juxtamembrane region is a critical determinant of SNAREdependent fusion. Mol Cell, 4, 415-21.

MEDINE, C. N., RICKMAN, C., CHAMBERLAIN, L. H. \& DUNCAN, R. R. 2007. Munc18-1 prevents the formation of ectopic SNARE complexes in living cells. J Cell Sci, 120, 4407-15.

MEEUSEN, S., MCCAFFERY, J. M. \& NUNNARI, J. 2004. Mitochondrial fusion intermediates revealed in vitro. Science, 305, 1747-52.

MIMA, J., HICKEY, C. M., XU, H., JUN, Y. \& WICKNER, W. 2008. Reconstituted membrane fusion requires regulatory lipids, SNAREs and synergistic SNARE chaperones. EMBO J, 27, 2031-42. 
MISURA, K. M., BOCK, J. B., GONZALEZ, L. C., JR., SCHELLER, R. H. \& WEIS, W. I. 2002. Threedimensional structure of the amino-terminal domain of syntaxin 6, a SNAP-25 C homolog. Proc Natl Acad Sci U S A, 99, 9184-9.

MISURA, K. M., GONZALEZ, L. C., JR., MAY, A. P., SCHELLER, R. H. \& WEIS, W. I. 2001a. Crystal structure and biophysical properties of a complex between the N-terminal SNARE region of SNAP25 and syntaxin 1a. J Biol Chem, 276, 41301-9.

MISURA, K. M., SCHELLER, R. H. \& WEIS, W. I. 2000. Three-dimensional structure of the neuronal-Sec1-syntaxin 1a complex. Nature, 404, 355-62.

MISURA, K. M., SCHELLER, R. H. \& WEIS, W. I. 2001b. Self-association of the H3 region of syntaxin 1A. Implications for intermediates in SNARE complex assembly. J Biol Chem, 276, 1327382.

MONTECUCCO, C., SCHIAVO, G. \& PANTANO, S. 2005. SNARE complexes and neuroexocytosis: how many, how close? Trends Biochem Sci, 30, 367-72.

MOSSESSOVA, E., BICKFORD, L. C. \& GOLDBERG, J. 2003. SNARE selectivity of the COPII coat. Cell, 114, 483-95.

MUNSON, M., CHEN, X., COCINA, A. E., SCHULTZ, S. M. \& HUGHSON, F. M. 2000. Interactions within the yeast t-SNARE Sso1p that control SNARE complex assembly. Nat Struct Biol, 7, 894-902.

NEHER, E. 1998. Vesicle pools and Ca2+ microdomains: new tools for understanding their roles in neurotransmitter release. Neuron, 20, 389-99.

NEWMAN, A. P., SHIM, J. \& FERRO-NOVICK, S. 1990. BET1, BOS1, and SEC22 are members of a group of interacting yeast genes required for transport from the endoplasmic reticulum to the Golgi complex. Mol Cell Biol, 10, 3405-14.

NICHOLSON, K. L., MUNSON, M., MILLER, R. B., FILIP, T. J., FAIRMAN, R. \& HUGHSON, F. M. 1998. Regulation of SNARE complex assembly by an N-terminal domain of the t-SNARE Sso1p. Nat Struct Biol, 5, 793-802.

NOVICK, P., FIELD, C. \& SCHEKMAN, R. 1980. Identification of 23 complementation groups required for post-translational events in the yeast secretory pathway. Cell, 21, 205-15. 
NOVICK, P. \& SCHEKMAN, R. 1979. Secretion and cell-surface growth are blocked in a temperature-sensitive mutant of Saccharomyces cerevisiae. Proc Natl Acad Sci U S A, 76, 1858-62.

OKAMOTO, M. \& SUDHOF, T. C. 1997. Mints, Munc18-interacting proteins in synaptic vesicle exocytosis. J Biol Chem, 272, 31459-64.

OSSIG, R., DASCHER, C., TREPTE, H. H., SCHMITT, H. D. \& GALLWITZ, D. 1991. The yeast SLY gene products, suppressors of defects in the essential GTP-binding Ypt1 protein, may act in endoplasmic reticulum-to-Golgi transport. Mol Cell Biol, 11, 2980-93.

OYLER, G. A., HIGGINS, G. A., HART, R. A., BATTENBERG, E., BILLINGSLEY, M., BLOOM, F. E. \& WILSON, M. C. 1989. The identification of a novel synaptosomal-associated protein, SNAP-25, differentially expressed by neuronal subpopulations. J Cell Biol, 109, 3039-52.

PACE, C. N., SHIRLEY, B. A., THOMSON, J.A. 1989. "Measuring the conformational stability of a protein", in CREIGHTON, T. E., Protein Structure: A Practical Approach, Oxford University Press, pp. 311-330.

PALADE, G. 1975. Intracellular aspects of the process of protein synthesis. Science, 189, 347-58.

PARLATI, F., MCNEW, J. A., FUKUDA, R., MILLER, R., SOLLNER, T. H. \& ROTHMAN, J. E. 2000. Topological restriction of SNARE-dependent membrane fusion. Nature, 407, 194-8.

PARLATI, F., VARLAMOV, O., PAZ, K., MCNEW, J. A., HURTADO, D., SOlLNER, T. H. \& ROTHMAN, J. E. 2002. Distinct SNARE complexes mediating membrane fusion in Golgi transport based on combinatorial specificity. Proc Natl Acad Sci U S A, 99, 5424-9.

PATEL, S. K., INDIG, F. E., OLIVIERI, N., LEVINE, N. D. \& LATTERICH, M. 1998. Organelle membrane fusion: a novel function for the syntaxin homolog Ufe1p in ER membrane fusion. Cell, 92, 611-20.

PAUMET, F., RAHIMIAN, V. \& ROTHMAN, J. E. 2004. The specificity of SNARE-dependent fusion is encoded in the SNARE motif. Proc Natl Acad Sci U S A, 101, 3376-80.

PELHAM, H. R. 1999. SNAREs and the secretory pathway-lessons from yeast. Exp Cell Res, 247, 18.

PENG, R. \& GALLWITZ, D. 2002. Sly1 protein bound to Golgi syntaxin Sed5p allows assembly and contributes to specificity of SNARE fusion complexes. J Cell Biol, 157, 645-55. 
PENG, R. \& GALLWITZ, D. 2004. Multiple SNARE interactions of an SM protein: Sed5p/Sly1p binding is dispensable for transport. EMBO J, 23, 3939-49.

PENG, R. W., GUETG, C., ABELLAN, E. \& FUSSENEGGER, M. 2010. Munc18b regulates core SNARE complex assembly and constitutive exocytosis by interacting with the N-peptide and the closed-conformation C-terminus of syntaxin 3. Biochem J, 431, 353-61.

PEVSNER, J., HSU, S. C., BRAUN, J. E., CALAKOS, N., TING, A. E., BENNETT, M. K. \& SCHELLER, R. H. 1994. Specificity and regulation of a synaptic vesicle docking complex. Neuron, $13,353-61$.

PFEFFER, S. \& AIVAZIAN, D. 2004. Targeting Rab GTPases to distinct membrane compartments. Nat Rev Mol Cell Biol, 5, 886-96.

PFEFFER, S. R. 1996. Transport vesicle docking: SNAREs and associates. Annu Rev Cell Dev Biol, 12, $441-61$.

POBBATI, A. V., STEIN, A. \& FASSHAUER, D. 2006. N- to C-terminal SNARE complex assembly promotes rapid membrane fusion. Science, 313, 673-6.

POIRIER, M. A., HAO, J. C., MALKUS, P. N., CHAN, C., MOORE, M. F., KING, D. S. \& BENNETT, M. K. 1998. Protease resistance of syntaxin.SNAP-25.VAMP complexes. Implications for assembly and structure. J Biol Chem, 273, 11370-7.

PRICE, A., SEALS, D., WICKNER, W. \& UNGERMANN, C. 2000. The docking stage of yeast vacuole fusion requires the transfer of proteins from a cis-SNARE complex to a Rab/Ypt protein. $J$ Cell Biol, 148, 1231-8.

REESE, C., HEISE, F. \& MAYER, A. 2005. Trans-SNARE pairing can precede a hemifusion intermediate in intracellular membrane fusion. Nature, 436, 410-4.

RICE, L. M., BRENNWALD, P. \& BRUNGER, A. T. 1997. Formation of a yeast SNARE complex is accompanied by significant structural changes. FEBS Lett, 415, 49-55.

RIENTO, K., KAUPPI, M., KERANEN, S. \& OLKKONEN, V. M. 2000. Munc18-2, a functional partner of syntaxin 3, controls apical membrane trafficking in epithelial cells. J Biol Chem, 275, 13476-83. 
RIZO, J., CHEN, X. \& ARAC, D. 2006. Unraveling the mechanisms of synaptotagmin and SNARE function in neurotransmitter release. Trends Cell Biol, 16, 339-50.

RIZO, J. \& ROSENMUND, C. 2008. Synaptic vesicle fusion. Nat Struct Mol Biol, 15, 665-74.

ROSSI, V., BANFIELD, D. K., VACCA, M., DIETRICH, L. E., UNGERMANN, C., D'ESPOSITO, M., GALLI, T. \& FILIPPINI, F. 2004. Longins and their longin domains: regulated SNAREs and multifunctional SNARE regulators. Trends Biochem Sci, 29, 682-8.

SACHER, M., BARROWMAN, J., SCHIELTZ, D., YATES, J. R., 3RD \& FERRO-NOVICK, S. 2000. Identification and characterization of five new subunits of TRAPP. Eur J Cell Biol, 79, 71-80.

SACHER, M., BARROWMAN, J., WANG, W., HORECKA, J., ZHANG, Y., PYPAERT, M. \& FERRO-NOVICK, S. 2001. TRAPP I implicated in the specificity of tethering in ER-to-Golgi transport. Mol Cell, 7, 433-42.

SACHER, M., JIANG, Y., BARROWMAN, J., SCARPA, A., BURSTON, J., ZHANG, L., SCHIELTZ, D., YATES, J. R., 3RD, ABELIOVICH, H. \& FERRO-NOVICK, S. 1998. TRAPP, a highly conserved novel complex on the cis-Golgi that mediates vesicle docking and fusion. EMBO J, 17, 2494-503.

SACHER, M., STONE, S. \& FERRO-NOVICK, S. 1997. The synaptobrevin-related domains of Bos1p and Sec22p bind to the syntaxin-like region of Sed5p. J Biol Chem, 272, 17134-8.

SASSA, T., HARADA, S., OGAWA, H., RAND, J. B., MARUYAMA, I. N. \& HOSONO, R. 1999. Regulation of the UNC-18-Caenorhabditis elegans syntaxin complex by UNC-13. J Neurosci, 19, $4772-7$.

SCHAGGER, H. \& VON JAGOW, G. 1987. Tricine-sodium dodecyl sulfate-polyacrylamide gel electrophoresis for the separation of proteins in the range from 1 to $100 \mathrm{kDa}$. Anal Biochem, 166, 36879.

SCHIAVO, G., BENFENATI, F., POULAIN, B., ROSSETTO, O., POLVERINO DE LAURETO, P., DASGUPTA, B. R. \& MONTECUCCO, C. 1992. Tetanus and botulinum-B neurotoxins block neurotransmitter release by proteolytic cleavage of synaptobrevin. Nature, 359, 832-5.

SCHIAVO, G., SANTUCCI, A., DASGUPTA, B. R., MEHTA, P. P., JONTES, J., BENFENATI, F., WILSON, M. C. \& MONTECUCCO, C. 1993. Botulinum neurotoxins serotypes A and E cleave SNAP-25 at distinct COOH-terminal peptide bonds. FEBS Lett, 335, 99-103. 
SCHOCH, S., DEAK, F., KONIGSTORFER, A., MOZHAYEVA, M., SARA, Y., SUDHOF, T. C. \& KAVALALI, E. T. 2001. SNARE function analyzed in synaptobrevin/VAMP knockout mice. Science, 294, 1117-22.

SCHUlZE, K. L., LITTLETON, J. T., SALZBERG, A., HALACHMI, N., STERN, M., LEV, Z. \& BELLEN, H. J. 1994. rop, a Drosophila homolog of yeast Sec1 and vertebrate n-Sec1/Munc-18 proteins, is a negative regulator of neurotransmitter release in vivo. Neuron, 13, 1099-108.

SCOTT, B. L., VAN KOMEN, J. S., IRSHAD, H., LIU, S., WILSON, K. A. \& MCNEW, J. A. 2004. Sec1p directly stimulates SNARE-mediated membrane fusion in vitro. J Cell Biol, 167, 75-85.

SEALS, D. F., EITZEN, G., MARGOLIS, N., WICKNER, W. T. \& PRICE, A. 2000. A Ypt/Rab effector complex containing the Sec1 homolog Vps33p is required for homotypic vacuole fusion. Proc Natl Acad Sci U S A, 97, 9402-7.

SHEN, J., TARESTE, D. C., PAUMET, F., ROTHMAN, J. E. \& MELIA, T. J. 2007. Selective activation of cognate SNAREpins by Sec1/Munc18 proteins. Cell, 128, 183-95.

SOGAARD, M., TANI, K., YE, R. R., GEROMANOS, S., TEMPST, P., KIRCHHAUSEN, T., ROTHMAN, J. E. \& SOLLNER, T. 1994. A rab protein is required for the assembly of SNARE complexes in the docking of transport vesicles. Cell, 78, 937-48.

SOLLNER, T., BENNETT, M. K., WHITEHEART, S. W., SCHELLER, R. H. \& ROTHMAN, J. E. 1993a. A protein assembly-disassembly pathway in vitro that may correspond to sequential steps of synaptic vesicle docking, activation, and fusion. Cell, 75, 409-18.

SOLLNER, T., WHITEHEART, S. W., BRUNNER, M., ERDJUMENT-BROMAGE, H., GEROMANOS, S., TEMPST, P. \& ROTHMAN, J. E. 1993b. SNAP receptors implicated in vesicle targeting and fusion. Nature, 362, 318-24.

SORENSEN, J. B., 2009. Conflicting views on the membrane fusion machinery and the fusion pore. Annu Rev Cell Dev Biol, 25, 513-37.

SORENSEN, J. B., NAGY, G., VAROQUEAUX, F., NEHRING, R. B., BROSE, N., WILSON, M. C. \& NEHER, E. 2003. Differential control of the releasable vesicle pools by SNAP-25 splice variants and SNAP-23. Cell, 114, 75-86.

SPANG, A. 2009. On vesicle formation and tethering in the ER-Golgi shuttle. Curr Opin Cell Biol, 21, 531-6. 
STARAI, V. J., HICKEY, C. M. \& WICKNER, W. 2008. HOPS proofreads the trans-SNARE complex for yeast vacuole fusion. Mol Biol Cell, 19, 2500-8.

STEIN, A., WEBER, G., WAHL, M. C. \& JAHN, R. 2009. Helical extension of the neuronal SNARE complex into the membrane. Nature, 460, 525-8.

STROP, P., KAISER, S. E., VRLJIC, M. \& BRUNGER, A. T. 2008. The structure of the yeast plasma membrane SNARE complex reveals destabilizing water-filled cavities. J Biol Chem, 283, 1113-9.

STROUPE, C., COLLINS, K. M., FRATTI, R. A. \& WICKNER, W. 2006. Purification of active HOPS complex reveals its affinities for phosphoinositides and the SNARE Vam7p. EMBO J, 25, 1579-89.

STROUPE, C., HICKEY, C. M., MIMA, J., BURFEIND, A. S. \& WICKNER, W. 2009. Minimal membrane docking requirements revealed by reconstitution of Rab GTPase-dependent membrane fusion from purified components. Proc Natl Acad Sci U S A, 106, 17626-33.

STRUTHERS, M. S., SHANKS, S. G., MACDONALD, C., CARPP, L. N., DROZDOWSKA, A. M., KIOUMOURTZOGLOU, D., FURGASON, M. L., MUNSON, M. \& BRYANT, N. J. 2009. Functional homology of mammalian syntaxin 16 and yeast Tlg2p reveals a conserved regulatory mechanism. $J$ Cell Sci, 122, 2292-9.

SUDHOF, T. C. \& ROTHMAN, J. E. 2009. Membrane fusion: grappling with SNARE and SM proteins. Science, 323, 474-7.

SUTTON, R. B., FASSHAUER, D., JAHN, R. \& BRUNGER, A. T. 1998. Crystal structure of a SNARE complex involved in synaptic exocytosis at 2.4 A resolution. Nature, 395, 347-53.

SZTUL, E., LUPASHIN, V. 2009. Role of vesicle tethering factors in the ER-Golgi membrane traffic. FEBS Lett, 583, 3770-83.

TELLAM, J. T., MCINTOSH, S. \& JAMES, D. E. 1995. Molecular identification of two novel Munc18 isoforms expressed in non-neuronal tissues. J Biol Chem, 270, 5857-63.

THURMOND, D. C., CERESA, B. P., OKADA, S., ELMENDORF, J. S., COKER, K. \& PESSIN, J. E. 1998. Regulation of insulin-stimulated GLUT4 translocation by Munc18c in 3T3L1 adipocytes. J Biol Chem, 273, 33876-83. 
THURMOND, D. C., KANZAKI, M., KHAN, A. H. \& PESSIN, J. E. 2000. Munc18c function is required for insulin-stimulated plasma membrane fusion of GLUT4 and insulin-responsive amino peptidase storage vesicles. Mol Cell Biol, 20, 379-88.

TITORENKO, V. I., CHAN, H. \& RACHUBINSKI, R. A. 2000. Fusion of small peroxisomal vesicles in vitro reconstructs an early step in the in vivo multistep peroxisome assembly pathway of Yarrowia lipolytica. J Cell Biol, 148, 29-44.

TITORENKO, V. I. \& RACHUBINSKI, R. A. 2000. Peroxisomal membrane fusion requires two AAA family ATPases, Pex1p and Pex6p. J Cell Biol, 150, 881-6.

TOCHIO, H., TSUI, M. M., BANFIELD, D. K. \& ZHANG, M. 2001. An autoinhibitory mechanism for nonsyntaxin SNARE proteins revealed by the structure of Ykt6p. Science, 293, 698-702.

TOGNERI, J., CHENG, Y. S., MUNSON, M., HUGHSON, F. M. \& CARR, C. M. 2006. Specific SNARE complex binding mode of the Sec1/Munc-18 protein, Sec1p. Proc Natl Acad Sci U S A, 103, 17730-5.

TOONEN, R. F., DE VRIES, K. J., ZALM, R., SUDHOF, T. C. \& VERHAGE, M. 2005. Munc18-1 stabilizes syntaxin 1 , but is not essential for syntaxin 1 targeting and SNARE complex formation. $J$ Neurochem, 93, 1393-400.

TOONEN, R. F. \& VERHAGE, M. 2003. Vesicle trafficking: pleasure and pain from SM genes. Trends Cell Biol, 13, 177-86.

TOONEN, R. F. \& VERHAGE, M. 2007. Munc18-1 in secretion: lonely Munc joins SNARE team and takes control. Trends Neurosci, 30, 564-72.

TSUI, M. M. \& BANFIELD, D. K. 2000. Yeast Golgi SNARE interactions are promiscuous. J Cell Sci, 113 ( Pt 1), 145-52.

TSUI, M. M., TAI, W. C. \& BANFIELD, D. K. 2001. Selective formation of Sed5p-containing SNARE complexes is mediated by combinatorial binding interactions. Mol Biol Cell, 12, 521-38.

VAN HOLDE, K. E., JOHNSON, W.C., HO, P. S. 2006. Principles of Physical Biochemistry, PrenticeHall, New Jersey. 
VAN DONGEN, E. M., EVERS, T. H., DEKKERS, L. M., MEIJER, E. W., KLOMP, L. W. \& MERKX, M. 2007. Variation of linker length in ratiometric fluorescent sensor proteins allows rational tuning of $\mathrm{Zn}(\mathrm{II})$ affinity in the picomolar to femtomolar range. J Am Chem Soc, 129, 3494-5.

VELAZQUEZ-CAMPOY, A. \& FREIRE, E. 2006. Isothermal titration calorimetry to determine association constants for high-affinity ligands. Nat Protoc, 1, 186-91.

VERHAGE, M., MAIA, A. S., PLOMP, J. J., BRUSSAARD, A. B., HEEROMA, J. H., VERMEER, H., TOONEN, R. F., HAMMER, R. E., VAN DEN BERG, T. K., MISSLER, M., GEUZE, H. J. \& SUDHOF, T. C. 2000. Synaptic assembly of the brain in the absence of neurotransmitter secretion. Science, 287, 864-9.

VOETS, T. 2000. Dissection of three Ca2+-dependent steps leading to secretion in chromaffin cells from mouse adrenal slices. Neuron, 28, 537-45.

VOETS, T., NEHER, E. \& MOSER, T. 1999. Mechanisms underlying phasic and sustained secretion in chromaffin cells from mouse adrenal slices. Neuron, 23, 607-15.

VOETS, T., TOONEN, R. F., BRIAN, E. C., DE WIT, H., MOSER, T., RETTIG, J., SUDHOF, T. C., NEHER, E. \& VERHAGE, M. 2001. Munc18-1 promotes large dense-core vesicle docking. Neuron, $31,581-91$.

WALCH-SOLIMENA, C., JAHN, R. \& SUDHOF, T. C. 1993. Synaptic vesicle proteins in exocytosis: what do we know? Curr Opin Neurobiol, 3, 329-36.

WANG, Y., DULUBOVA, I., RIZO, J. \& SUDHOF, T. C. 2001. Functional analysis of conserved structural elements in yeast syntaxin Vam3p. J Biol Chem, 276, 28598-605.

WASHBOURNE, P., THOMPSON, P. M., CARTA, M., COSTA, E. T., MATHEWS, J. R., LOPEZBENDITO, G., MOLNAR, Z., BECHER, M. W., VALENZUELA, C. F., PARTRIDGE, L. D. \& WILSON, M. C. 2002. Genetic ablation of the t-SNARE SNAP-25 distinguishes mechanisms of neuroexocytosis. Nat Neurosci, 5, 19-26.

WEBER, T., ZEMELMAN, B. V., MCNEW, J. A., WESTERMANN, B., GMACHL, M., PARLATI, F., SOLLNER, T. H. \& ROTHMAN, J. E. 1998. SNAREpins: minimal machinery for membrane fusion. Cell, 92, 759-72. 
WEBER, M., CHERNOV, K., TURAKAINEN, H., WOHLFAHRT, G., PAJUNEN, M., SAVILAHTI, H. \& JANTTI, J. 2010. Mso1p regulates membrane fusion through interactions with the putative Npeptide-binding area in Sec1p domain 1. Mol Biol Cell, 21, 1362-74.

WEBER-BOYVAT, M., ARO, N., CHERNOV, K. G., NYMAN, T. \& JANTTI, J. 2011. Sec1p and Mso1p C-terminal tails cooperate with the SNAREs and Sec4p in polarized exocytosis. Mol Biol Cell, 22, 230-44.

WEIMER, R. M., RICHMOND, J. E., DAVIS, W. S., HADWIGER, G., NONET, M. L. \& JORGENSEN, E. M. 2003. Defects in synaptic vesicle docking in unc-18 mutants. Nat Neurosci, 6 , 1023-30.

WENINGER, K., BOWEN, M. E., CHOI, U. B., CHU, S. \& BRUNGER, A. T. 2008. Accessory proteins stabilize the acceptor complex for synaptobrevin, the 1:1 syntaxin/SNAP-25 complex. Structure, 16, 308-20.

WHITEHEART, S. W., SCHRAW, T. \& MATVEEVA, E. A. 2001. N-ethylmaleimide sensitive factor (NSF) structure and function. Int Rev Cytol, 207, 71-112.

WHYTE, J. R., MUNRO, S. 2002. Vesicle tethering complexes in membrane traffic. J Cell Sci, 115, 2627-37.

WILLIAMS, A. L., EHM, S., JACOBSON, N. C., XU, D. \& HAY, J. C. 2004. rsly1 binding to syntaxin 5 is required for endoplasmic reticulum-to-Golgi transport but does not promote SNARE motif accessibility. Mol Biol Cell, 15, 162-75.

WIEDERHOLD, K., KLOEPPER, T. H., WALTER, A. M., STEIN, A., KIENLE, N., SORENSEN, J. B. \& FASSHAUER, D. 2010. A coiled coil trigger site is essential for rapid binding of synaptobrevin to the SNARE acceptor complex. J Biol Chem, 285, 21549-59.

WONG, P. P., DANEMAN, N., VOLCHUK, A., LASSAM, N., WILSON, M. C., KLIP, A. \& TRIMBLE, W. S. 1997. Tissue distribution of SNAP-23 and its subcellular localization in 3T3-L1 cells. Biochem Biophys Res Commun, 230, 64-8.

WU, M. N., SCHULZE, K. L., LLOYD, T. E. \& BELLEN, H. J. 2001. The ROP-syntaxin interaction inhibits neurotransmitter release. Eur J Cell Biol, 80, 196-9.

XIAO, W., POIRIER, M. A., BENNETT, M. K. \& SHIN, Y. K. 2001. The neuronal t-SNARE complex is a parallel four-helix bundle. Nat Struct Biol, 8, 308-11. 
XU, T., BINZ, T., NIEMANN, H. \& NEHER, E. 1998. Multiple kinetic components of exocytosis distinguished by neurotoxin sensitivity. Nat Neurosci, 1, 192-200.

XU, T., RAMMNER, B., MARGITTAI, M., ARTALEJO, A. R., NEHER, E. \& JAHN, R. 1999. Inhibition of SNARE complex assembly differentially affects kinetic components of exocytosis. Cell, 99, 713-22.

XU, Y., SU, L. \& RIZO, J. 2010. Binding of Munc18-1 to synaptobrevin and to the SNARE four-helix bundle. Biochemistry, 49, 1568-76.

XU, Y., ZHANG, F., SU, Z., MCNEW, J. A. \& SHIN, Y. K. 2005. Hemifusion in SNARE-mediated membrane fusion. Nat Struct Mol Biol, 12, 417-22.

YAMAGUCHI, T., DULUBOVA, I., MIN, S. W., CHEN, X., RIZO, J. \& SUDHOF, T. C. 2002. Sly1 binds to Golgi and ER syntaxins via a conserved N-terminal peptide motif. Dev Cell, 2, 295-305.

YANG, B., GONZALEZ, L., JR., PREKERIS, R., STEEGMAIER, M., ADVANI, R. J. \& SCHELLER, R. H. 1999. SNARE interactions are not selective. Implications for membrane fusion specificity. J Biol Chem, 274, 5649-53.

YANG, B., STEEGMAIER, M., GONZALEZ, L. C., JR. \& SCHELLER, R. H. 2000. nSec1 binds a closed conformation of syntaxin1A. J Cell Biol, 148, 247-52.

ZHANG, F., CHEN, Y., KWEON, D. H., KIM, C. S. \& SHIN, Y. K. 2002. The four-helix bundle of the neuronal target membrane SNARE complex is neither disordered in the middle nor uncoiled at the C-terminal region. J Biol Chem, 277, 24294-8.

ZHANG, Y., SU, Z., ZHANG, F., CHEN, Y. \& SHIN, Y. K. 2005. A partially zipped SNARE complex stabilized by the membrane. J Biol Chem, 280, 15595-600.

ZWILLING, D., CYPIONKA, A., POHL, W. H., FASSHAUER, D., WALLA, P. J., WAHL, M. C. \& JAHN, R. 2007. Early endosomal SNAREs form a structurally conserved SNARE complex and fuse liposomes with multiple topologies. EMBO J, 26, 9-18. 


\section{Appendix}

\section{A.1 Sequence alignment of Sed5p from fungi}
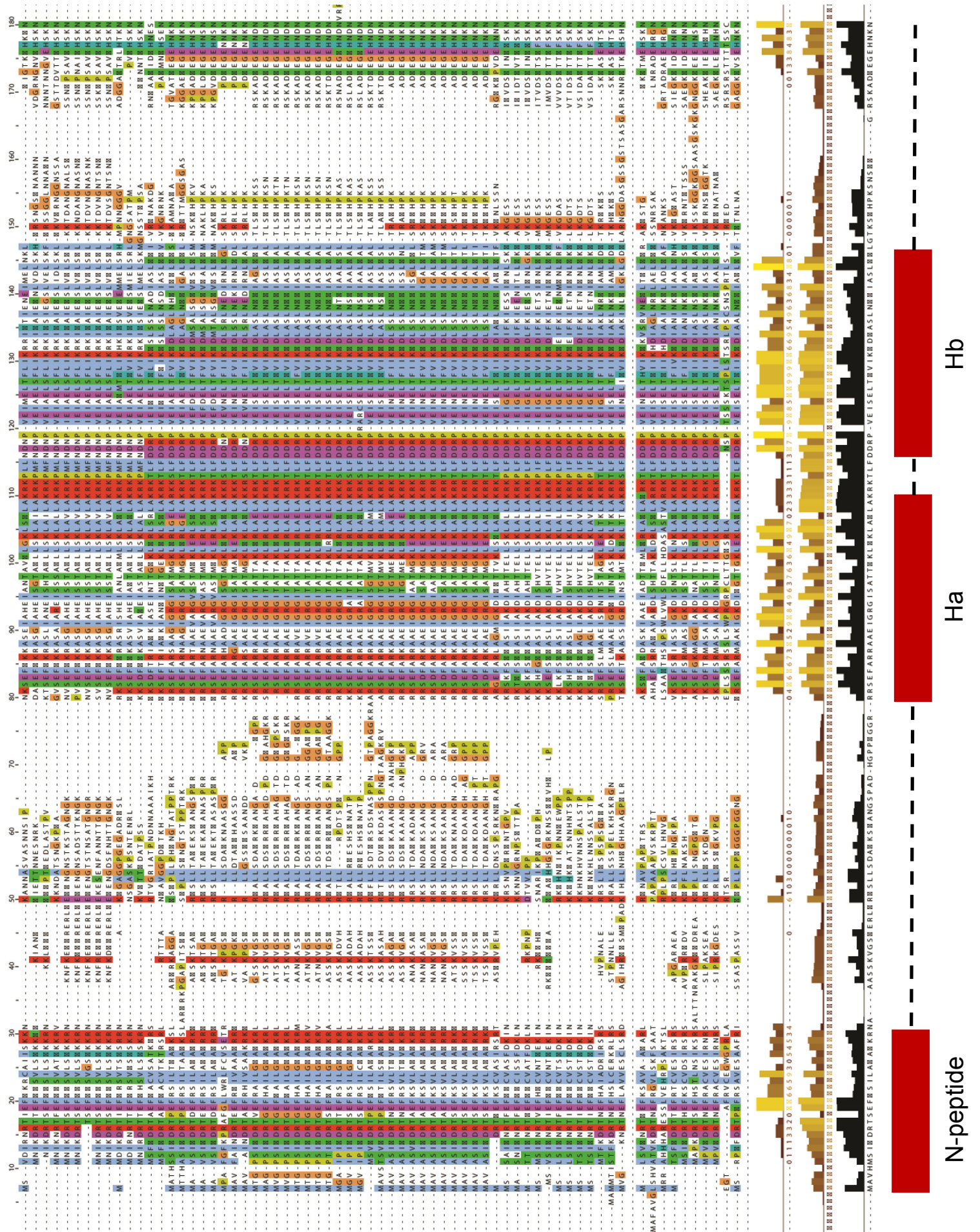

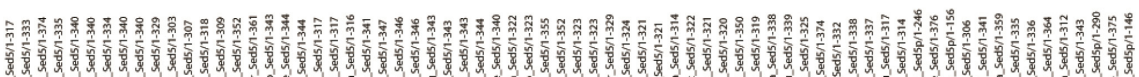

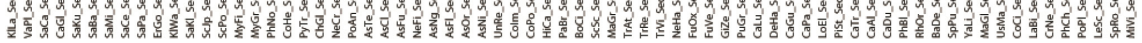




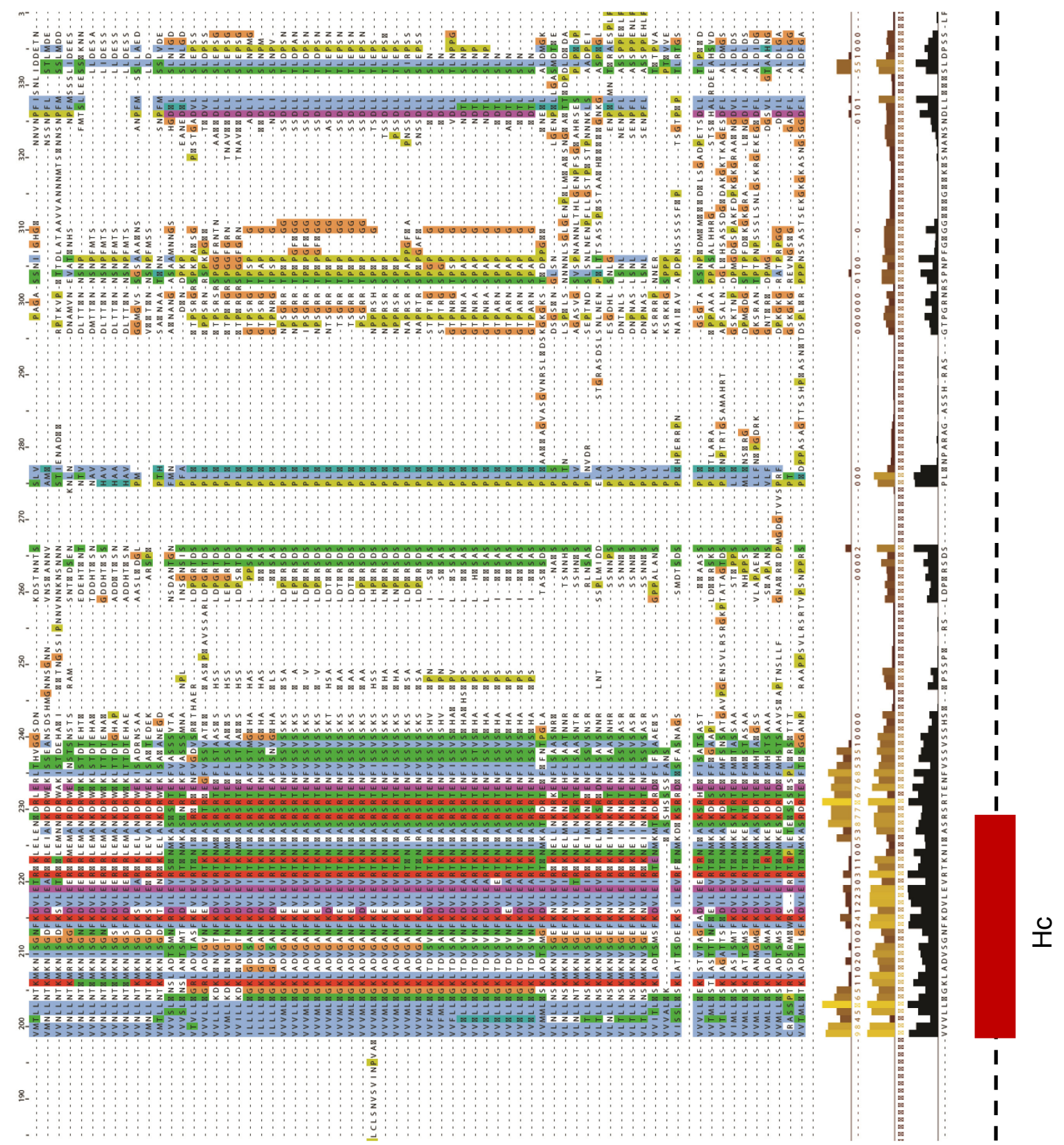




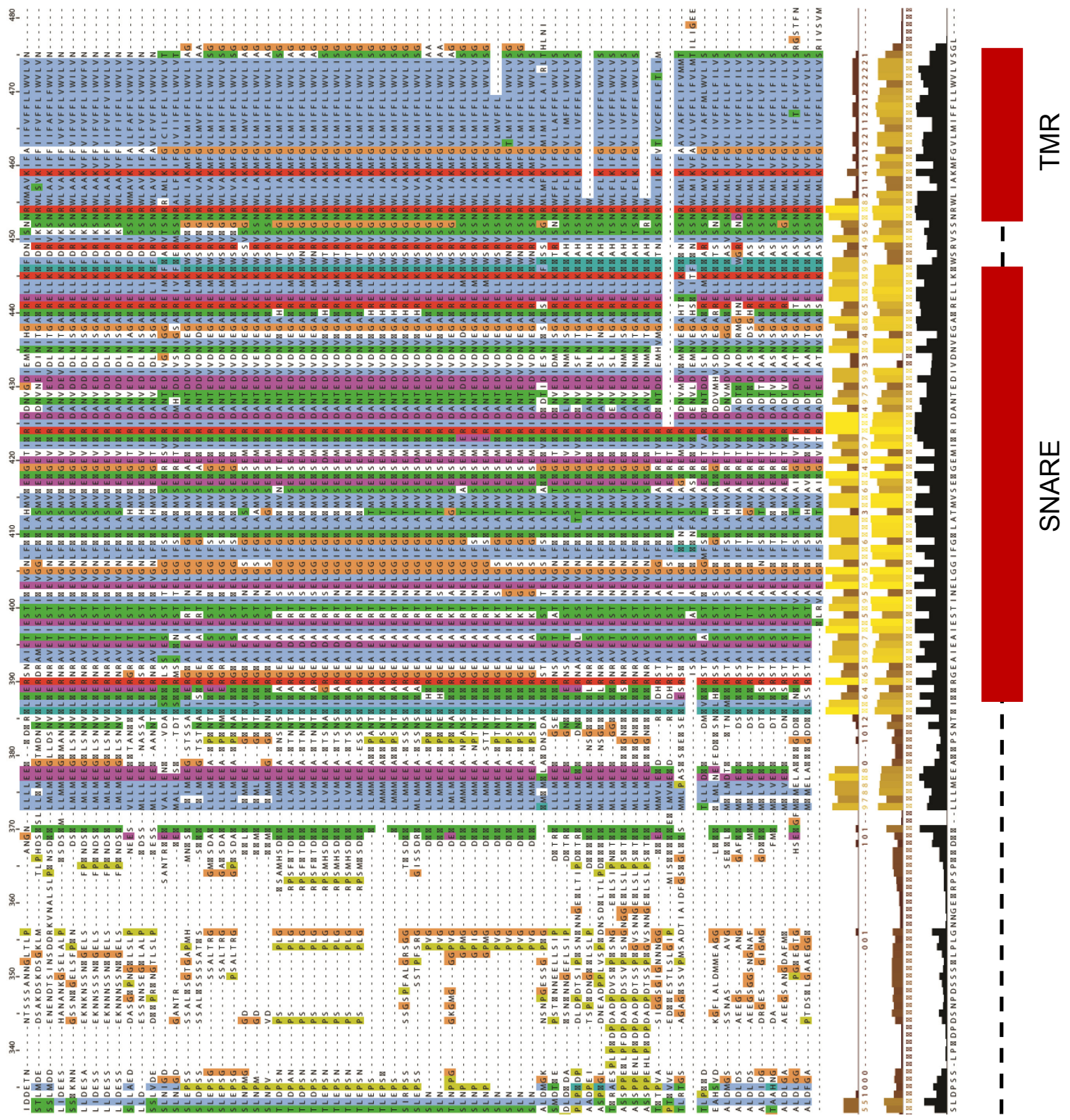

Figure A.1: Sequence alignment of Sed5p from fungi

The protein sequence of Sed5p from several fungi species was aligned and kindly provided by Dr. Nickias Kienle. The output was generated using JALVIEW and divided into three parts shown on different pages. On the bottom of the panels, domain organization of Sed5p, composed of the N-peptide, the Habc domain, the SNARE motif and the transmembrane region (TMR), is depicted according to their approximate positions on the alignment. The dashed lines represent the linker regions between domains. 


\section{A.2 Purification of stable complexes formed between the ER-Golgi} SNAREs

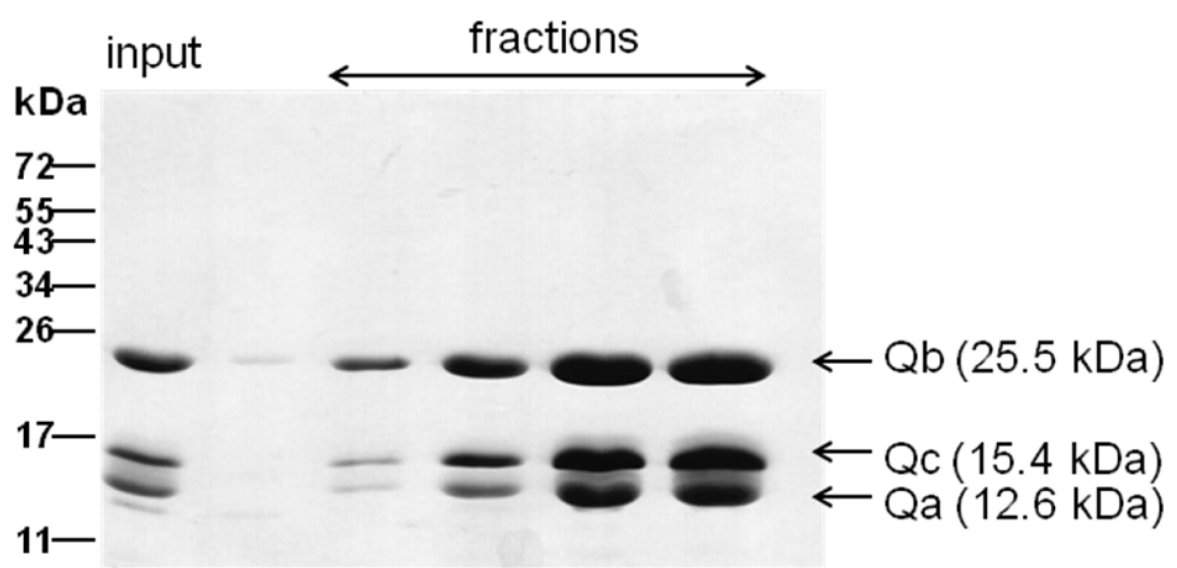

kDa

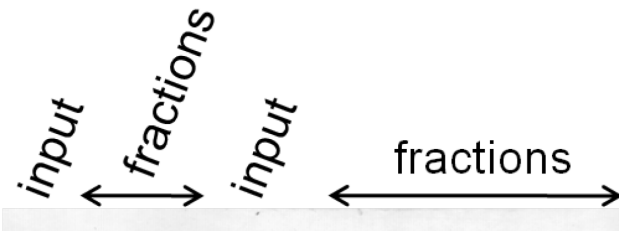

$55.4-$

$36.5-$

$21.5-$

$14.4-$

6
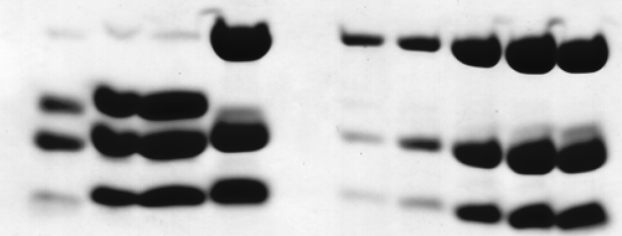

$\leftarrow \mathrm{Qb}(25.5 \mathrm{kDa})$

$\leftarrow Q_{c}(15.4 \mathrm{kDa})$

$\leftarrow$ Qa $(12.6 \mathrm{kDa})$

$3.5-$

$\leftarrow \mathrm{R}(7.1 \mathrm{kDa})$

Figure A.2: Purification of stable complexes formed between the ER-Golgi SNAREs

The ER-Golgi SNAREs Sed5p (Qa), Bos1p (Qb), Bet1p (Qc) and Sec22p (R) were purified as described in section 2.2.1. In order to assemble the Qabc, QabR and QacR complexes, equimolar amounts of the individual proteins were mixed in the presence of $500 \mathrm{mM} \mathrm{NaCl}$ and incubated overnight. Next, excess $\mathrm{NaCl}$ was removed via dialysis and the monomers were separated from the complexes by using ion-exchange chromatography (MonoQ HR 5/5). The protein complexes were eluted by using a linear $\mathrm{NaCl}$ gradient and the peak fractions were analysed by SDS-PAGE gel electrophoresis. On the upper part, SDS-PAGE gel analysis of the Qabc complex and on the lower part, SDS-PAGE gel analysis of the QabR and QacR complexes are shown. 


\section{A.3 Sly1p does not enhance the assembly kinetics of the Qabc and the QacR complexes}
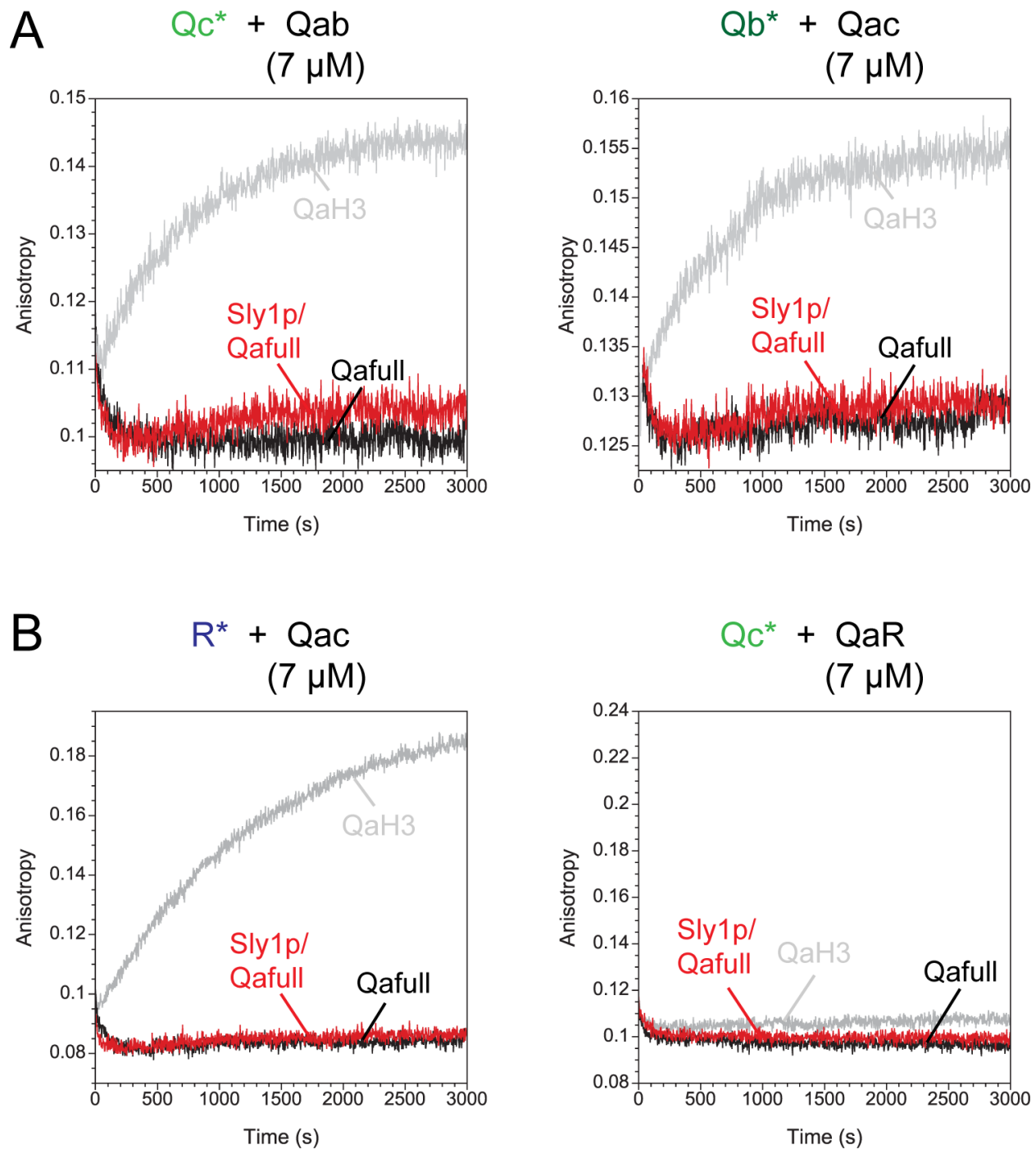

Figure A.3: Sly1p does not enhance the assembly kinetics of the Qabc (A) and the QacR (B) complexes

Assembly of the Qabc (A) and QacR (B) complexes was followed by mixing $~ 400 \mathrm{nM}$ fluorescent SNAREs with $\sim 7 \mu \mathrm{M}$ of their unlabeled partner SNAREs. The unlabeled proteins were pre-incubated before the experiments. Either QaH3 (grey curves) or Qafull (black curves) were used, the latter also in the presence of $\sim 11 \mu \mathrm{M}$ of Sly1p (red curves). Each experiment was performed in an ultra-micro fluorescence cell, in a total volume of $180 \mu \mathrm{l}$. A. Assembly of the Qabc complex was monitored, either by mixing fluorescent Qc* (Bet1p C85-OG) with Qa- and Qb-SNAREs (plot on the left); or by mixing fluorescent Qb* (Bos1p C181-OG) with Qaand Qc-SNAREs (plot on the right). B. Similar to A, assembly of the QacR complex was monitored, either by mixing fluorescent $R^{*}$ (Sec22p C131-OG) with Qa- and Qc-SNAREs (plot on the left); or by mixing fluorescent Qc* (Bet1p C85-OG), with Qa- and R-SNAREs (plot on the right). Note that, QacR-complexes do not assemble when Qa- and R-SNAREs are pre-incubated. 


\section{Curriculum Vitae}

\section{F. ESRA DEMIRCIOGLU}

Max Planck Institute for Biophysical Chemistry

Department of Neurobiology

Am Fassberg 11, 37077

Goettingen, Germany

Phone: +49551 2011624

Fax: +495512011499

e-mail: edemirc@gwdg.de

Nationality: Turkish

Date of Birth: 19.04.1983

Place of Birth: Bursa, Turkey

\section{EDUCATION}

10/ 2007 - 11/ 2011 Max Planck Institute for Biophysical Chemistry, Goettingen, Germany

Pursuing PhD degree in Structural Biochemistry

09/ 2006 - 04/ 2008 International Max Planck Research School, Goettingen, Germany MSc/ PhD program of Molecular Biology

Earned a MSc degree in Molecular Biology (Master's project integrated into the first six months of the PhD project)

Overall grade of the Master's examinations: 1.54 (B - very good)

09/ 2001 - 06/ 2006 Middle East Technical University (METU), Ankara, Turkey

Earned a BSc degree in Molecular Biology and Genetics

C. GPA: 3.81 out of 4.00

\section{PRACTICAL EXPERIENCE}

10/ 2007 - present Max Planck Institute for Biophysical Chemistry,

Goettingen, Germany

PhD project: Comparative biophysical and biochemical studies on SNARE/ SM protein interactions

Supervisor: Dr. Dirk Fasshauer (currently Prof. in University of Lausanne

Methods: Molecular cloning \& site-directed mutagenesis, protein 
expression \& purification, Isothermal Titration Calorimetry (ITC), fluorescence spectroscopy (anisotropy and FRET), CD spectroscopy, protein-labeling for EPR spectroscopy

05/ 2007 - 07/ 2007 Max Planck Institute for Biophysical Chemistry, Goettingen, Germany

Laboratory rotation in the department of Prof. Dr. Reinhard

Lührmann

Project: Potential roles of Cyclophilins in spliceosomal network

Methods: Cell culture, fluorescence in situ hybridization (FISH),

immunofluorescence, immunoprecipitation, in vitro splicing assays

01/ 2007 - 03/ 2007 Goettingen Centre for Molecular Biosciences (GZMB),

Goettingen, Germany

Laboratory rotation in the department of Prof. Dr. Ralf Ficner

Project: Structural investigation of $\operatorname{Imp} \beta / \operatorname{Imp} 7$ cooperativity for $\mathrm{H} 1$ import and Ran-independence of Imp $\beta /$ Snurportin1

Methods: Protein expression and purification, X-Ray crystallography

07/ 2005 - 09/ 2005 FLI-Leibniz Institute for Age Research, Jena, Germany

Summer practice in the laboratory of Prof. Dr. Christoph Englert

Project: Localization of an enhancer element for $w t 1$ gene in zebrafish

Methods: Maintenance of zebrafish, in situ hybridization methods, microinjection

01/ 2004 - 05/ 2004 Middle East Technical University (METU), Ankara, Turkey

Practical experience in the laboratory of Prof. Dr. Feride Severcan

Project: Impact of melatonin on the rat brain tissue

Methods: Fourier Transform Infrared Spectroscopy (FTIR)

\section{PUBLICATIONS}

Demircioglu F. E., Fasshauer D. "Sly1p binds not only the N-terminal peptide, but also the remainder of Sed5p" Manuscript in preparation.

Demircioglu F. E., Fasshauer D. "Sly1p promotes a transient intermediate complex which allows the rapid assembly of ER-Golgi SNAREs" Manuscript in preparation.

\section{AWARDS \& DISTINCTIONS}

$04 / 2011$

$07 / 2010$

$12 / 2008$
Invited speaker of the $8^{\text {th }}$ Molecular mechanisms of Exocytosis \& Endocytosis meeting in Edinburgh, Scotland Attended the 35th FEBS Congress in Stockholm, Sweden GGNB (Goettingen Graduate Schools for Neurosciences and Molecular Biosciences) Excellence Fellowship for PhD studies 
07/ 2008

Attended the abc6 Applications of Biocalorimetry in Heidelberg,

Germany (ITC Conferences \& Workshop from MicroCal)

\section{TEACHING}

10/ $2009-04 / 2010$ BSc thesis project of Dennis Addo-Nkansah from Stenden Hogeschool in Emmen, the Netherlands

03/ 2009 - 05/ 2009 Laboratory rotation project of Helena Magliarelli from the MSc/PhD program of Molecular Biology in Göttingen, Germany

05/ $2008-07 / 2008$ Laboratory rotation project of Alwaleed Alkhaja from the MSc/ PhD program of Molecular Biology in Goettingen, Germany

\section{SKILLS \& INTERESTS}

$\begin{array}{ll}\text { Languages } & \text { English: Advanced in written and spoken } \\ & \text { German: Intermediate in written } \\ & \text { Turkish: Mother tongue } \\ \text { Computer skills } & \text { Microsoft Office, PyMOL, KaleidaGraph } \\ \text { Hobbies } & \text { Violin, yoga }\end{array}$

\section{REFEREES}

\section{1) Prof. Dr. Dirk Fasshauer}

Department of Cell Biology and Morphology

Faculty of Biology and Medicine

University of Lausanne

Rue du Bugnon 9

Room 330

1005 Lausanne/ Switzerland

Phone: +41- 216925287

e-mail: Dirk.Fasshauer@unil.ch

\section{2) Prof. Dr. Reinhard Jahn}

Max Planck Institute for Biophysical

Chemistry

Department of Neurobiology

Am Fassberg 11

37077

Göttingen/Germany

Phone: +49551 2011635

e-mail: rjahn@gwdg.de

\section{3) Prof. Dr. Markus C. Wahl}

Free University of Berlin

Faculty of Biology, Chemistry and Pharmacy

Institute for Chemistry and Biochemistry

Takustr. 6

D-14195

Berlin/ Germany

Phone: +49 (0)30 838-53456

e-mail: mwahl@chemie.fu-berlin.de 


\section{List of publications}

Demircioglu F. E., Fasshauer D. "Sly1p binds not only the N-terminal peptide, but also the remainder of Sed5p" Manuscript in preparation.

Demircioglu F. E., Fasshauer D. "Sly1p promotes a transient intermediate complex which allows the rapid assembly of ER-Golgi SNAREs" Manuscript in preparation. 